\title{
Kinetics of structure formation in block copolymers
}

\author{
Dissertation \\ zur Erlangung des mathematisch-naturwissenschaftlichen \\ Doktorgrades \\ "Doctor rerum naturalium" \\ der Georg-August-Universität Göttingen \\ im Promotionsprogramm ProPhys \\ der Georg-August University School of Science (GAUSS)
}

\author{
vorgelegt von \\ Yongzhi REN
}

aus Heilongjiang, China

Göttingen, April 2018 


\section{Betreuungsausschuss}

Marcus Müller Institut für Theoretische Physik, Georg-August-Universität Göttingen

Annette Zippelius Institut für Theoretische Physik, Georg-August-Universität Göttingen

\section{Mitglieder der Prüfungskommission}

Referent: Marcus Müller Institut für Theoretische Physik, Georg-August-Universität Göttingen

Korreferentin: Annette Zippelius Institut für Theoretische Physik, Georg-AugustUniversität Göttingen

\section{Weitere Mitglieder der Prüfungskommission:}

Jörg Enderlein III. Physikalisches Institut, Georg-August-Universität Göttingen Matthias Krüger Institut für Theoretische Physik, Georg-August-Universität Göttingen

Philipp Vana Institut für Physikalische Chemie, Georg-August-Universität Göttingen

Stefan Klumpp Institut für Nichtlineare Dynamik, Georg-August-Universität Göttingen

Tag der mündlichen Prüfung: 
GEORG-AUGUST-UNIVERSITÄT GÖTTINGEN

\section{Abstract}

Fakultät für Physik

Institut für Theoretische Physik

by Yongzhi REN 
Block copolymers are amphiphilic macromolecules, which consist of multiple incompatible parts. Although block copolymers may differ in their microscopic interactions, their phase behaviors share many similarities. In equilibrium, block copolymers self-assemble into various spatially modulated phases with long-range order whose free energy may differ only a small fraction of thermal energy per molecule.

This characteristic makes the understanding of the structure formation both interesting and challenging. In this thesis, we investigate the structure formation of block copolymers on the basis of the free-energy landscape. We develop numerical schemes to investigate two important quantities: One is the thermodynamic forces that drive the formation of the patterns. The other is the Onsager coefficient that translates the thermodynamic forces into the flow of the particles.

The calculation of the thermodynamic forces is indirect and computational demanding in particle-based simulations. The most efficient approach is probably the field-theoretic umbrella sampling method. We propose an equivalent approach in the framework of the self-consistent field theory (SCFT), which significantly improves the computational efficiency and accuracy.

The Onsager coefficient connects the single-chain dynamics with the co-operative movement of many molecules. An analytical form of the Onsager coefficient is difficult to obtain. We propose a numerical scheme to directly measure Onsager coefficients in particle-based simulations. To be specific, we measure the Onsager coefficient in symmetric homopolymer blends. We find that the singlepolymer dynamics and the kinetics of collective variables are highly correlated. As a result, on very short time and length scales, the Onsager coefficient is a time-dependent variable, which differs from the prediction of the Rouse model.

The structure formation of block copolymers is an important and multi-faceted research topic. We focus on the structure formation process in a quasi-twodimensional system of symmetric diblock copolymer melts. When the system is quenched far below the order-disorder transition temperature, the relaxation of the structure towards long-range order is very protracted because it involves numerous thermally activated processes that alter the topology of the microphase-separated morphology. The free-energy landscape of the system is rugged and it has been likened to that of glass-forming systems. 
Using large-scale particle-based simulations we study the kinetics of structure formation in symmetric lamella-forming diblock copolymers after a quench from the disordered state. We characterize the ordering process by the correlation length of the lamellar structure and its Euler characteristics. The latter integral-geometry morphological measure indicates changes of the structure topology and allows us to identify defects.

The density fields of snapshots of the particle-based simulations are used as starting values for SCFT calculations. The latter converge to a local, metastable minimum of the free-energy landscape. This combination of particle-based simulation and SCFT calculations allows us to relate an instantaneous configuration of the particle-based model to a corresponding metastable free-energy minimum of SCFT, and we typically observe that a change of the metastable state is associated with a change of the Euler characteristics of the particle-based morphology, i.e., changes of free-energy basins are correlated to changes of the domain topology.

Additionally, we employ the string method in conjunction with the SCFT to study the free-energy barriers and minimum free-energy paths (MFEP) involved in changes of the domain topology.

By a combination study of the free-energy landscape and the Onsager coefficient that connects the thermodynamic force with the polymer dynamics, we obtain a complete description of the density evolution dynamics. By a comparison to the particle-based simulations, our findings capture essential properties which allow us to predict the kinetics of structure formation in many nanostructure-forming systems. 


\section{Acknowledgements}

Firstly, I would like to express my sincere gratitude to my supervisor Prof. Marcus Müller for his patient training in my Ph.D. study and related research. His guidance and continuous support helped me a lot in all the time of research and writing of this thesis.

Secondly, I would like to appreciate my colleges and friends in my institute. Their accompany gives me a meaningful and joyful time in the science city of Göttingen. Especially, I would like to thank Prof. Weihua Li, who gives me clear guidance when I was fresh. I also would like to thank Prof. Guojie Zhang, Dr. Dewen Sun and Dr. Qiyun Tang for fruitful and inspiring discussions.

Financial support by the China Scholarship Council (CSC) grants is gratefully acknowledged. Computing in the JSC Jülich, is also gratefully acknowledged.

Finally and most importantly, I want to thank my parents and my wife, their supports are my ultimate impetus. 


\section{Contents}

$\begin{array}{ll}\text { Abstract } & \text { ii }\end{array}$

$\begin{array}{ll}\text { Acknowledgements } & \text { v }\end{array}$

$\begin{array}{ll}\text { List of Figures } & \text { ix }\end{array}$

List of Tables $\quad$ xii

Abbreviations $\quad$ xiii

1 Introduction 1

1.1 Background ...................... 1

1.2 Kinetics of structure formation in diblock copolymer melts . . . . 4

1.3 Theoretical models . . . . . . . . . . . . . . . . . . . 7

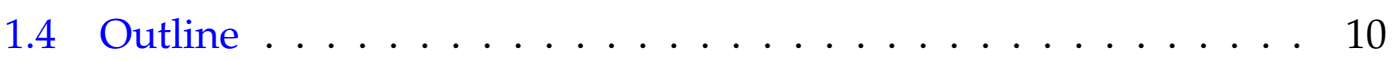

2 Model and Computational Techniques $\quad \mathbf{1 2}$

2.1 Phase separation and microphase separation . . . . . . . . . . . 12

2.2 Soft, particle-based, coarse-grained model . . . . . . . . . . . . . . 15

2.2.1 Bonded interaction . . . . . . . . . . . . . . . 15

2.2.2 Non-bonded interaction . . . . . . . . . . . . . . 16

2.3 Particle-based simulation . . . . . . . . . . . . . . . . . 17

2.3.1 Rouse dynamics . . . . . . . . . . . . . . 18

2.3.2 Smart Monte Carlo algorithm . . . . . . . . . . . . . 20

2.3.3 Single-Chain-in-Mean-Field (SCMF) algorithm . . . . . . . 23

2.3.3.1 Performance and workflow . . . . . . . . 27

2.3.4 A comparison of the SCMF algorithm with analytical results 30

2.3.4.1 Mean-square center-of-mass displacement in isotropic, homogeneous system . . . . . . . . . . 30

2.3.4.2 Expansion of an isolated polymer chain . . . . 32

2.3.4.3 Order-disorder transition of diblock copolymer

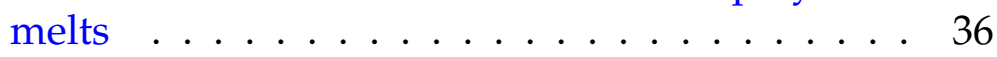

2.4 Self-consistent field theory . . . . . . . . . . . . . . . 39 
2.4.1 Method . . . . . . . . . . . . . . . . . . . 40

2.4.2 Numerical implementation . . . . . . . . . . . . . . . 45

2.4 .3 Performance . . . . . . . . . . . . . . . . . . . 48

2.5 A comparison of the particle-based SCMF algorithm simulation to the SCFT method . . . . . . . . . . . . . . . . . . 50

2.5.1 AB binary homopolymer blends . . . . . . . . . . 50

3 Free-energy landscape of block copolymers 56

3.1 Free-energy functionals . . . . . . . . . . . . . . . . . 56

3.2 Numerical derivation of the chemical potential . . . . . . . . . 59

3.2.1 Random-phase approximation . . . . . . . . . . . . 59

3.2.2 Exact, delta-function constraint in SCFT . . . . . . . . . 60

3.2.3 Umbrella potential method in SCFT . . . . . . . . . . . . 62

3.2.4 Field-theoretic umbrella sampling in the particle-based sim-

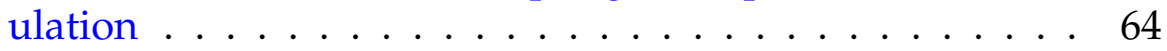

3.2.5 Numerical results . . . . . . . . . . . . . . . . . . . . 65

3.2.5.1 A comparison between the RPA method and the exact results obtained by the delta-function constraint in conjunction with SCFT . . . . . . . . 65

3.2.5.2 An analysis of the umbrella potential method in conjunction with SCFT . . . . . . . . . . 67

3.2.5.3 A comparison of the umbrella potential method in SCFT and the field-theoretic umbrella sampling method in conjunction with the SCMF algorithm . . . . . . . . . . . . 69

3.3 Dynamic evolution of densities . . . . . . . . . . . . . . 72

3.3.1 Conserved dynamics . . . . . . . . . . . . . . . . 73

3.3.2 External potential dynamics (EPD) . . . . . . . . . . . . 73

3.4 A direct measurement of Onsager coefficients in particle-based simulations . . . . . . . . . . . . . . . . 76

3.4.1 Onsager coefficient: the long-time limit . . . . . . . . . 79

3.4.2 Evidence of time-dependent behavior for the Onsager coefficient: numerical results in the high-frequency regime . 82

3.4 .3 Conclusions . . . . . . . . . . . . . . . . . . . 85

3.5 Minimum free-energy path $\ldots \ldots \ldots \ldots \ldots \ldots$

3.5.1 Illustration of string method . . . . . . . . . . . 86

3.5.2 Orientation transition of lamellar-forming diblock copolymers . . . . . . . . . . . . . . . 88

3.5.3 Impact of the molecular architecture on the structure for-

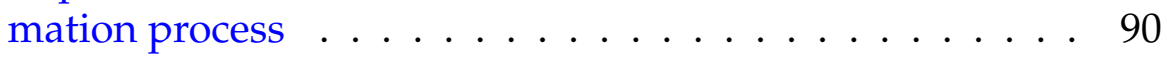

4 Pattern formation in diblock copolymer melts $\quad 94$

4.1 Qualitative behavior of diblock copolymers in a thin film . . . . 994

4.2 Commensurability and lamellar spacing . . . . . . . . . . . . 97

4.3 Correlation length . . . . . . . . . . . . . . . . . 100 
4.4 Identifying free-energy basins: Inherent morphology and Euler characteristics . . . . . . . . . . . . . 106

4.4.1 Integral-geometry morphological analysis . . . . . . . . . . 106

4.4 .2 Identifying free-energy basins . . . . . . . . . . . . . . . . 108

4.5 Defects . . . . . . . . . . . . . . . . 116

4.5.1 Approximately estimating the defect density via the Euler characteristic of small patches . . . . . . . . . . . 116

4.5.2 Quantifying the ordering process: time evolution of defect density . . . . . . . . . . . . . . . . 119

4.5.3 Minimum free-energy path of two typical defects . . . . 122

4.5.3.1 Bifurcation in the free-energy landscape . . . . . 122

4.5.3.2 Trifurcation in the free-energy landscape . . . . . 126

5 Summary and outlook $\quad 130$

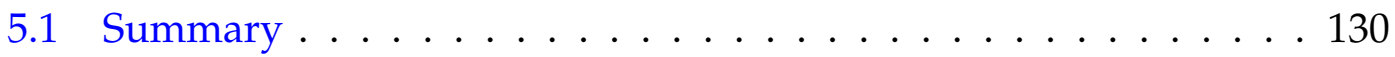

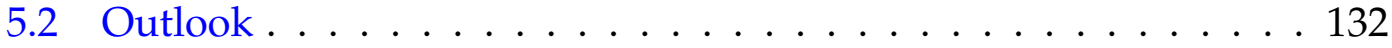

$\begin{array}{ll}\text { A Other umbrella potentials } & 133\end{array}$

$\begin{array}{ll}\text { B Random-phase approximation } & 136\end{array}$

$\begin{array}{lr}\text { Bibliography } & 143\end{array}$ 


\section{List of Figures}

1.1 Scheme representation of various spatially modulated, ordered

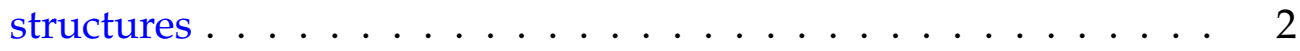

1.2 Scheme representation of various types of block copolymers . . . 3

1.3 The phase diagram and equilibrium morphologies of diblock copolymer melts in the bulk. . . . . . . . . . . . . . . . . . . 4

1.4 SEM images of block copolymer PS-b-PMMA films annealed at different temperatures . . . . . . . . . . . . . 5

1.5 Phenomena of polymers on different length scales and time scales 8

2.1 Mean-square center-of-mass displacement, $g_{3}$, per SMC step as a function of the time step length, $\Delta t \ldots \ldots \ldots 22$

2.2 The flowchart of the SCMF algorithm . . . . . . . . . . . . . 28

2.3 Performance of the SCMF algorithm on a single GPU . . . . . . . 29

2.4 Mean-square, center-of-mass displacement, $g_{3}$ and mean-square displacement of segments, $g_{1}$ as a function of simulation time $t$. . 31

2.5 Mean-square end-to-end distance, $R_{\mathrm{e} 0}^{2}$ as a function of $\kappa N$. . . . 33

2.6 Mean-square end to end distance, $R_{\mathrm{e} 0}^{2}$ as a function of external fields update frequency. . . . . . . . . . . . . . . 34

2.7 The reciprocal of the peak value of the structure factor of composition as a function of the incompatible parameter, $\chi N \ldots \ldots 36$

2.8 The flowchart of the SCFT algorithm . . . . . . . . . . . . . . 45

2.9 A comparison of the density Profile obtained from the SCFT and the SCMF particle-based simulation. . . . . . . . . . . . 51

2.10 Phase transition of symmetric binary homopolymer blend . . . . 53

2.11 The interfacial width as a function of $\chi N \ldots \ldots 55$

3.1 A comparison between the exact chemical potential and the RPA results . . . . . . . . . . . . . . . . 6 66

3.2 The density profile of diblock copolymers with a sharp interface. 68

3.3 A comparison of chemical potentials obtained by the umbrella potential method to the exact results for block copolymers. . . . . 69

3.4 A comparison between the free energy obtained by the umbrella potential method and the exact results for block copolymers. . . . 70

3.5 The chemical potential of diblock copolymers computed by the field-theoretic umbrella sampling method . . . . . . . . . . 71

3.6 The time-evolution of various density profile with different Fourier modes . . . . . . . . . . . . . . . . . . . . . 83 
3.7 The time-evolution of density profile amplitude, $A(q, t)$ at $q R_{\mathrm{e} 0} / 2 \pi=$ 1 and $q R_{\mathrm{e} 0} / 2 \pi=1.5$, respectively. . . . . . . . . . 84

3.8 The snapshots of the MFEP that connects the metastable, layered lamellae to the stable, standing lamellae. . . . . . . . . . . . . 88

3.9 The free-energy difference of the states on MFEP. . . . . . . . . . . 89

3.10 Defect removal of the B-core dislocation dipole in diblock copolymers. . . . . . . . . . . . . . . . . . . . 91

3.11 Defect removal of the B-core dislocation dipole in triblock copolymers. . . . . . . . . . . . . . . . . 92

4.1 The time evolution of diblock copolymer melts in a thin film at $\chi N=15$ and $\chi N=20$, respectively. . . . . . . . 95

4.2 Free energies of morphologies with different lamellar spacing. . . 98

4.3 The optimal lamellar spacing as a function of the Florry-Huggins parameter $\chi N \ldots \ldots \ldots \ldots$. . . . . . . . . . . . 99

4.4 The structure factors of the composition, $S(\mathbf{q})$ in the symmetric diblock copolymer thin film . . . . . . . . . . . . . . . . . . 101

4.5 The correlation function, $g_{r}$ is obtained as a function of $\Delta r$ for six independent simulations with different $\chi N \ldots \ldots$. . . . . . 102

4.6 The correlation length, $\xi$ at $\chi N=16$ and $\chi N=20$ with different system size L. . . . . . . . . . . . . . . . . . . . . . . . 104

4.7 The correlation length, $\xi$ at different $\chi N$ as a function of simulation time t. . . . . . . . . . . . . . . . 105

4.8 The time evolution of the domain area and the domain perimeter. 108

4.9 First example of inherent morphologies. . . . . . . . . . . . . . . . 110

4.10 Second example of inherent morphologies. . . . . . . . . . . . . . 113

4.11 The time dependence of the persistence rate of Euler characteristic $\Pi(11.23 \tau) \ldots \ldots$. . . . . . . . . . . . . . . . 115

4.12 Snapshot of various defect in a typical morphology obtained by the particle-based simulation. . . . . . . . . . . . . . . 117

4.13 Snapshots of three typical defects. . . . . . . . . . . . . . 118

4.14 Counting defects in a typical two-dimensional fingerprint morphology. . . . . . . . . . . . . . . . . . . . 119

4.15 Estimate the density of of local defect motifs as a function of time $t$ after a quench to $\chi N=20$ with different system size. . . . . . . 120

4.16 Estimate the density of of local defect motifs as a function of time $t$ after a quench to $\chi N=18,20$, and $30 \ldots \ldots 121$

4.17 Estimate the density of of isolated domain motifs as a function of time $t$ after a quench to $\chi N=18,20$, and $30 \ldots \ldots$. . . . . . 122

4.18 Snapshot of particle-based simulation and MFEP for an intersection defect. . . . . . . . . . . . . . . . . . . . . . . 123

4.19 Snapshot and MFEP of a B-core dislocation dipole like defect annihilation process. . . . . . . . . . . . . . . . . 125

4.20 The MFEPs to eliminate an isolated block domain. . . . . . . . . . 127 
4.21 The statistics of defect annihilation processes in the particle-based simulations. . . . . . . . . . . . . . . . . 127

B.1 The single-chain correlation function . . . . . . . . . . . . . . 139 


\section{List of Tables}

2.1 Performance of the SCMF algorithm on a single GPU core. . . . . 30

2.2 Performance of the SCMF algorithm on multiple GPUs. . . . . . . 30

2.3 Performance of the SCFT algorithm on a single GPU. . . . . . . . 49

2.4 Performance of multiple SCFT calculations on a single GPU . . . 49

2.5 Performance of the SCFT algorithm on multiple CPUs . . . . . . 50 


\section{Abbreviations}

CPU Central Processing Unit

CUDA Compute Unified Device Architecture

DSA Directed Self-Assembly

GPU Graphics Processing Unit

MPI Message Passing Interface

MFEP Minimum Free- Energy Path

ODT Order-Disorder Transition

SAXS Small-Angle $X$-ray Scattering

SCFT Self Consistent-Field Theory

SCMF Single-Chain-in-Mean-Field algorithm

SMC Smart Monte Carlo 
Dedicated to my parents and wife. 


\section{Chapter 1}

\section{Introduction}

\subsection{Background}

The advance of material science has significantly changed the world. The human history is divided into eras named after the materials that are used; the Stone Age, the Bronze Age, and the Iron Age. Nowadays polymers are one of the most important materials. Polymers are large molecules composed of many repeated subunits which are called monomers [1]. For example, polymers such as polyethylene (PE), polystyrene (PS), polypropylene (PP) are used in daily life as plastics.

There are many fascinating properties, which make polymers so popular. One of the most attractive features of polymers is that block copolymer materials, which are constructed by two or more different monomers can spontaneously self-assemble into many spatially modulated, ordered structures below their critical temperatures (See Fig. 1.1). This feature is especially important for hightech industries (e.g., the aviation industry, the pharmaceutical industry, and the transport industry). Because the fabrication of functional materials with a crystal structure, which has a feature size ranging from nanometer to micrometer is important for many applications including lithographic templates of highdensity magnetic storage media, quantum dots, catalysis scaffolds and etc [35]. This feature also enabled a diverse and expanding range of practical applications in, e.g., the drug delivery [6], microelectronic materials [7], and advanced plastics [8]. 


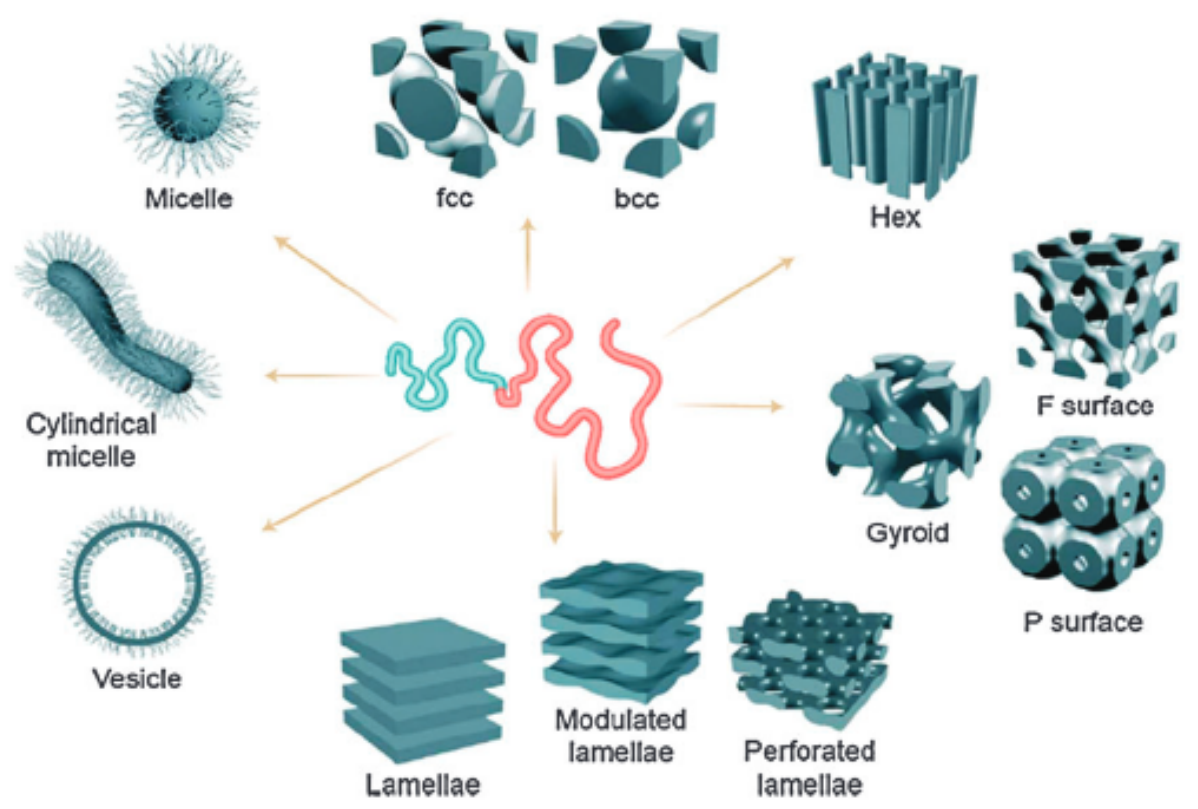

FIGURE 1.1: Scheme representation of various spatially modulated, ordered structures formed by block copolymers, including spherical and cylindrical micelles, spheres with face-centered cubic (space group: $F m \overline{3} m$ ) and bodycentered cubic packing $(\operatorname{Im} \overline{3} \mathrm{~m})$, hexagonally packed cylinders $(p 6 m)$, bicontinuous gyroid $(I a \overline{3} d)$, F surface $(F d \overline{3} \mathrm{~m})$, P surface $(\operatorname{Pm} \overline{3} n, P n \overline{3} m$, or $\operatorname{Pm} \overline{3} m)$, and lamella. The figure is obtained with permission from ref. [2].

Various nanoscale structures are accessible by synthesizing special polymer architectures. Recent investigations demonstrate that the formation of many novel nonclassical spherical packing phases (e.g., the complex Frank-Kasper phases) could be attributed to three factors: the conformational asymmetry between the different blocks, the local segregation of the block copolymers, and the architecture of the block copolymers $[9,10]$.

In practical experiments, modern synthetic techniques can access a broad portfolio of multiblock molecular architectures [11]. As shown in Fig. 1.2, it is possible to prepare diblock, triblock, multiblock, and starblock copolymers. By using the technique of living anionic polymerization, it is possible to manufacture polymers with a narrow distribution of molecular weights [12].

On one hand, the complexity of block copolymer architectures allows us to access a huge amount of possible nanoscale patterns. On the other hand, the exploration of such a large parameter space by experimental methods becomes formidable. Fortunately, with the development of modern computer techniques, 


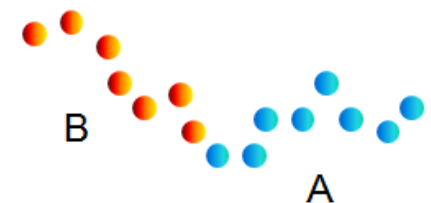

A

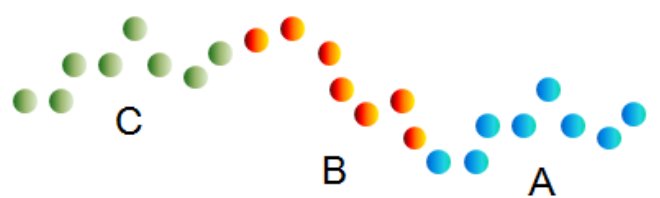

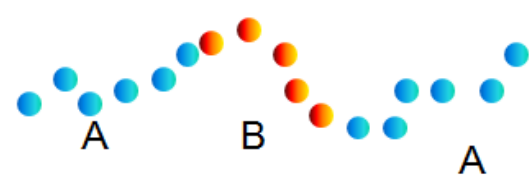

A

FIGURE 1.2: Scheme representation of various types of block copolymers. Upper left: $\mathrm{AB}$ diblock copolymer. Upper right: $\mathrm{ABC}$ triblock copolymer. Lower left: $\mathrm{ABC}$ triblock copolymer. Lower right: $\mathrm{AB}$-type multiblock copolymers.

the effort to explore these complex systems is becoming more and more affordable. By establishing different levels of theoretical models, it is possible to simulate and predict phenomena on different time and length scales. For example, the typical time constant that captures the properties dominated by the local vibrations of bond angles is about $10^{-13} s$ [13]. On a more coarse-grained, microscopic level, atomistic details are not important. Usually, a self-assembly process of block copolymers is characterized by the intrinsic width of interfaces between different domains, which are on the order of nanometers. Meanwhile, a self-assembly process may take several hundred seconds in experiments [14].

Modern computational technologies provide a profound insight. By modern computational techniques (e.g., the numerical self-consistent field theory), it is not difficult to extract equilibrium information of block copolymers. However, to understand the kinetic processes of structure formation is still challenging. In this dissertation, we focus on the kinetics of ordering in block copolymers, especially the ordering process that ensures after a quench of block copolymer melts from a disordered state to a microphase-separated, ordered structure.

In the following, we will review current approaches to the structure formation process in block copolymers. Specifically, we focus on the ordering process in lamellar-forming block copolymers in a thin film. In this thesis, a thin film is defined as a quasi-two-dimensional system whose thickness is smaller than the typical extension of lamellar structures (i.e., the lamellar spacing). Apart from conventional applications such as membranes, lithography, and coatings 
(1)
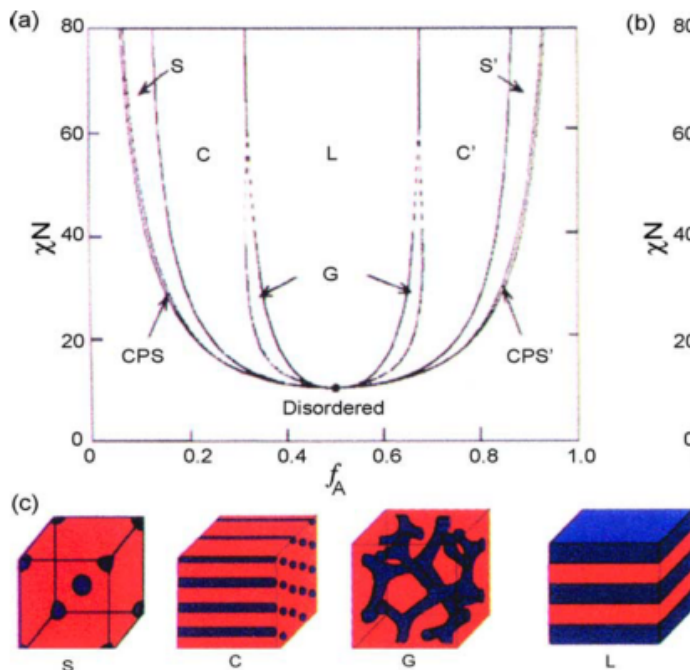

L

(b)
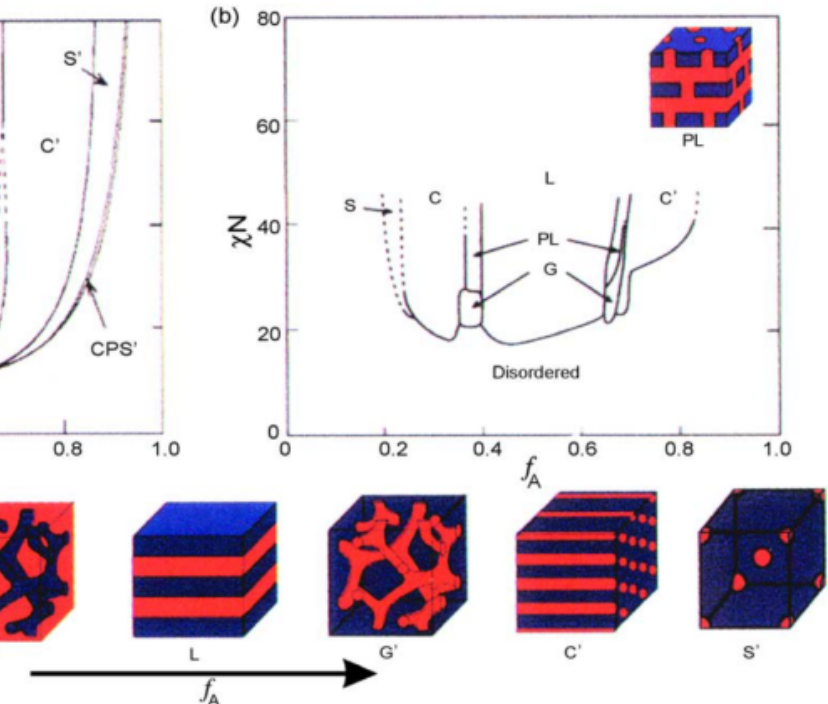

FIGURE 1.3: The phase diagram and equilibrium morphologies of diblock copolymer melts in the bulk. (a) $S$ and $S^{\prime}=$ body-centered-cubic spheres, $C$ and $C^{\prime}=$ hexagonally packed cylinders, $G$ and $G^{\prime}=$ bicontinuous gyroids, and $L=$ lamellae. Theoretical phase diagram of $\mathrm{AB}$ diblock copolymer is predicted by the self-consistent-field theory, depending on volume fraction $f_{A}$ of the blocks and the segregation parameter, $\chi N ; C P S$ and $C P S^{\prime}=$ closely packed spheres. (b) Theoretical phase diagram. (c) Experimental phase diagram of polyisoprene-block-polystyrene copolymer melts. The figure is obtained with permission from ref. [18].

[15], polymer thin films are of important interest for device technologies such as light-emitting diodes, photodiodes and thin film transistors [16].

\subsection{Kinetics of structure formation in diblock copoly- mer melts}

In equilibrium, the melt of linear $A B$ diblock copolymers typically exhibits body-centered-cubic, hexagonally ordered cylinder, lamellar, and several bicontinuous, e.g, gyroid structures. The phase diagram of diblock copolymer melts can be obtained by the SCFT method as shown in Fig. 1.3 [17, 18]. $f_{A}$ is the volume fraction of the A block. $\chi N$ is the production of the Flory-Huggins interaction, $\chi$, which is temperature dependent and the total degree of polymerization, $N$. 


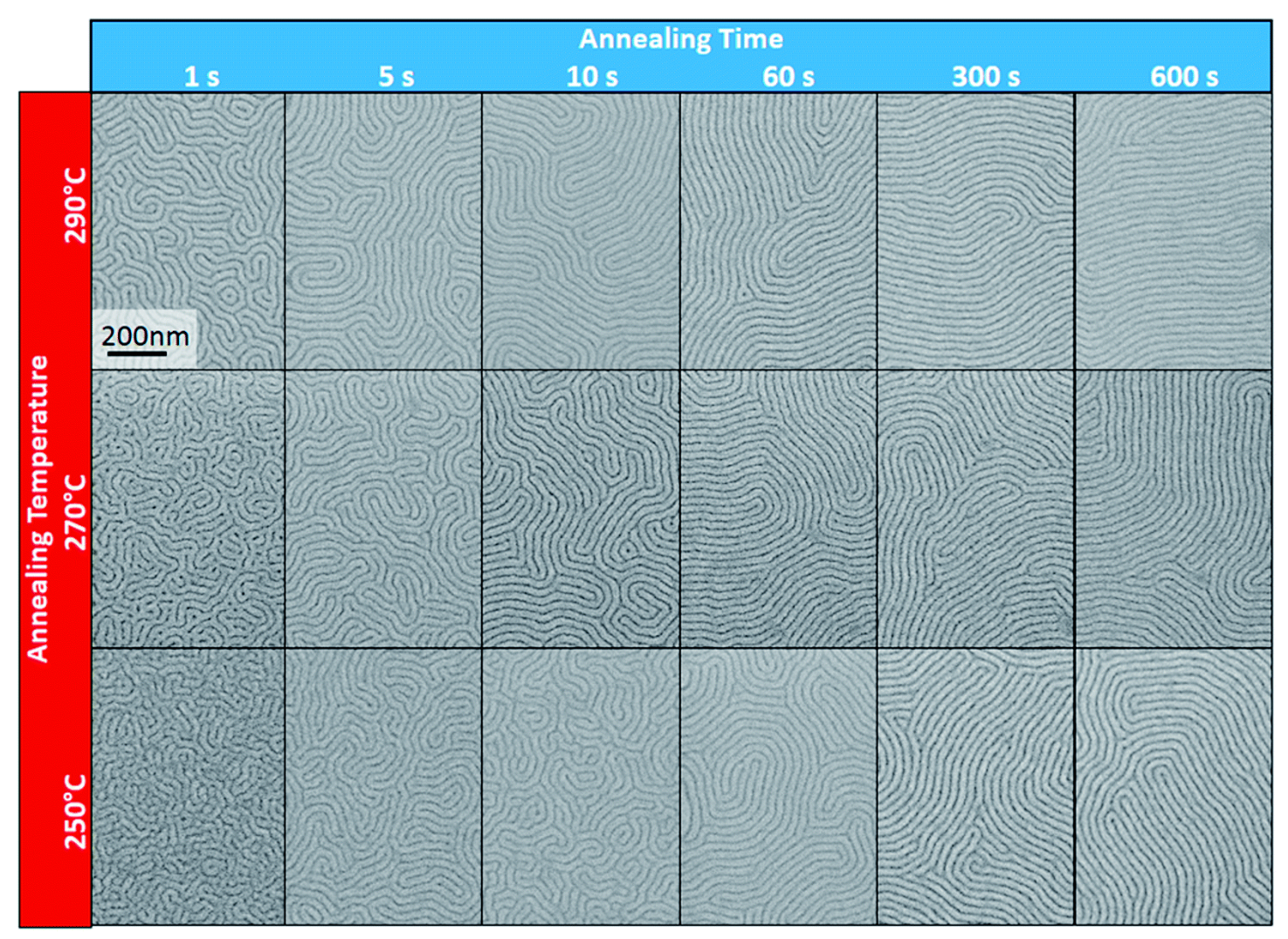

FIGURE 1.4: Plan view Scanning Electron Microscopy (SEM) images of block copolymer, polystyrene-b-polymethylmethacrylate (PS-b-PMMA) films annealed at different temperatures $\left(T=250,270\right.$, and $290^{\circ} \mathrm{C}$.) for different time periods (from 1 to $600 \mathrm{~s}$ ). The images were acquired after oxygen plasmaetching steps for $60 \mathrm{~s}$ in order to remove the PMMA phase and enhance the contrast. The block copolymer PS-b-PMMA with a styrene fraction of $f_{A}=0.5$ (symmetric copolymer). This image is obtained with permission from ref. [14].

These ordered structures are expected to be the thermodynamical equilibrium state. In our study, we quench the symmetric diblock copolymer melts from a homogeneous, disordered, high-temperature state to a specific temperature which is below the order-disorder transition temperature characterized by the incompatibility parameter $\chi N$. As shown in the phase diagram in Fig. 1.3, the critical point of the symmetric $\mathrm{AB}$ diblock copolymer melts is at $\chi N \approx 10.495$ [19].

In the absence of external guiding fields, the kinetics of microphase separation that ensues after a quench from the disordered state below the order-disorder transition typically does not result in an almost defect-free structure with a few isolated defects. Instead, a fingerprint-like morphology that is riddled with defects is formed in experiments and computer simulations [20-22] (See Fig. 1.4). 
A fingerprint-like morphology is locally ordered arranged with the lamellar order, i.e., a parallel arrangement of internal AB interfaces that extends over a few lamellae. On large scales, the small lamellar grains differ in their orientation and positional registration. These fingerprint-like morphologies are isotropic with a small characteristic length scale which depends on the simulation time and the temperature.

The defect density is so large that the idealized notion of well-defined defects that weakly interact breaks down, and the changes of morphology are rather co-operative. Nevertheless, the multitude of metastable fingerprint morphologies indicates that the free-energy landscape is rugged. Intriguingly, Zhang and Wang have likened the rugged free-energy functional to that of glass-forming systems and estimated the location of a glass transition using field-theoretic techniques [23]. The glass-transition is characterized by a divergence of the relaxation time scale [23-25]. The concept of the glass in the structure formation is different from the polymer glass [26]. In a polymer glass, the viscosity (or the relaxation time) is divergent below the critical glass transition temperature $T_{g}$. The long relaxation time is due to the slow dynamics of atomistic segments. Here, the slowness does not refer to the polymer chain motion but to the time evolution of collective morphology.

The structure formation can be categorized into three, qualitatively different stages: (i) The initial, homogeneous state is spinodally unstable and thermal concentration fluctuations grow exponentially in time giving rise to a highly irregular domain morphology, i.e., the fingerprint structure. In the course of this initial spinodal self-assembly, the concentration inside the microphase-separated domains reaches its equilibrium value but no long-range order is established [27]. (ii) In the subsequent stage - local topology changes and grain formation - the topology of the structure gradually evolves towards local lamellar order via thermally activated processes that alter the domain connectivity. At the end of this stage, the merging and separation of domains results in the formation of multiple lamellar grains. These grains differ in their orientation and are separated by grain boundaries. Importantly, there is a scale separation between the size of a grain, in which lamellar order is established, and the width of a grain boundary. (iii) In the final stage of ordering - grain coarsening - the grain boundaries move so that larger grains grow and smaller ones shrink. The growth of the average grain size or correlation length, $\xi$, is described by a power 
law in time, $\xi \sim t^{\eta}$. In related systems, a growth exponent with a value $\eta \approx 0.25$ has been observed [28].

Above discussions suggest that without external guiding fields, experiments and computer simulations usually obtain a defective morphology. For the purposes of industrial applications, much effort has been made to reduce the defect densities by external chemical or topographical guiding fields (directed selfassembly, DSA) [29].

There are several different directed self-assembly methods to manufacture largescale, defect-free, geometrical, structures. Shear is frequently utilized to align block copolymer domains in thin films [30-33]. Electric field can also be applied to develop long-range order [34-36]. These two methods do not manipulate the structure with an explicit guidance on the nanometer length scale. Thus it is difficult to fabricate patterns with an extensively low defect density.

On the microscopic level, one can use short-range guiding fields to control the structure formation. One technique is called graphoepitaxy, which uses topologically sculptured substrates [37]. The other strategy is called chemoepitaxy, which employs chemically patterned substrates [38]. Both strategies are low cost and easy to be integrated into the conventional lithographic process.

To efficiently devise strategies for defect removal, one important approach is to calculate the minimum free-energy path (MFEP) [39]. The MFEP is the most probable transition path to eliminate defects. With the help of the string method which is the most efficient method to calculate the MFEP, people are able to understand how the defects become unstable by various directed self-assembly methods.

\subsection{Theoretical models}

The study of polymeric systems can be carried out at different levels of description depending on the length scales and time scales as shown in Fig. 1.5.

At the most fundamental level, people employ a fully atomistic model for a polymer melt. Every atom is considered and they interact with each other with a finely tunned parametrization of the interaction force fields. The interactions 


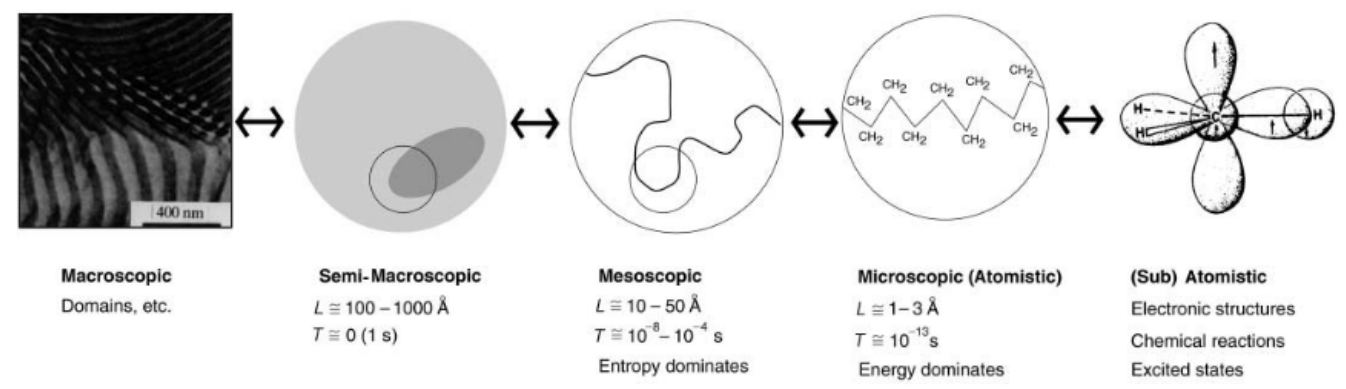

FIGURE 1.5: Phenomena of polymers on different length scales and time scales. This image is obtained with permission from ref. [13].

between a pair of neutral atoms or molecules can be simplified by the LennardJones model [40].

Quantum effects account for electronic structures, chemical reactions, and et al. On the atomistic length scale, quantum effects are usually not considered because the de Broglie wavelength of a carbon atom is much smaller than the typical length of a carbon atom. Meanwhile, all quantum simulations are very expensive and limited, e.g., the Car-Parrinello density functional simulation and the path-integral quantum Monte Carlo simulations [41, 42].

In a classical description, depending on the chemical complexity of the molecule, one considers the bonded interactions which determine the chemical structure of the molecule and non-bonded interactions. The bonded interactions contain terms accounting for specific chemical bonds which may include the bondbending, dihedral, and torsion angle potentials. The non-bonded interactions include Van der Waals forces and other electrostatic interactions [43, 44].

The aim of an atomistic simulation or a molecular simulation is to describe the local properties of a molecule with great precision. The time scale of an atomistic simulation is usually on the order of a few nanoseconds and the length scale ranges from $1-10 \mathrm{~nm}$ [42]. In the study of the kinetics of structure formation in block copolymers, it is not possible nor necessary to investigate a fully atomic model which contains all degrees of freedom of the particles. The length scale of a self-assembly usually ranges from $1 \mathrm{~nm}$ to $1000 \mathrm{~nm}$. The typical time scale to describe a structure formation process usually takes several hundred seconds. To increase the time scale and the length scale of a simulation, a coarse-graining procedure is a necessity. 
The basic idea to derive a coarse-grained model is by grouping a number of atoms in a chemically realistic description of an effective interaction (called segment). The mapping from an atomic model to a mesoscale model makes it possible to access the time and length scale that are far beyond atomistic simulations. A review of mapping electronic to macroscopic degrees of freedom is presented in [45]. Many collective phenomena (e.g., the structure formation) can be captured by coarse-grained models.

In a bottom-up construction, one can derive the coarse-grained model from an underlying microscopic model [46]. The partition function of the coarsegrained model and the microscopic model are enforced to be identical. One can derive the coarse-grained model by integrating out all the microscopic degrees of freedom. This bottom-up approach resembles renormalization theory exploiting the fractal Gaussian structure of a polymer in a dense melt [47].

An alternative approach is the physically motivated top-down approach, where we construct a minimal model with only relevant parameters to bring about the self-assembly of block copolymers. Although block copolymers may differ in their microscopic interactions, their physical behaviors share many similarities. For instance, the mean-square end-to-end distance of a polymer chain in a melt can be expressed as $\left\langle R_{\mathrm{e}}^{2}\right\rangle \propto N^{2 \nu}$. $\nu$ is a dimensionless exponent which is independent of the specific form of the short-range interactions that connect the beads and only depends on the dimension of the system. Because of the self-similar structure of the long, flexible polymer chain, there is a separation between the atomistic structure of a chemical monomeric repeat unit and the size of an entire macromolecule. By the renormalization group calculations, there are few relevant properties to bring about the universal behavior of the self-assembly [47], e.g., the connectivity along the macromolecular backbone, the repulsive force between unlike species, and the limited compressibility of the dense polymer melts. A more detailed description of the top-down, coarsegrained model is presented in the chapter 2.

On a mesoscopic scale description, the continuum field models achieved success in the study of the self-assembly of block copolymer melts or blends. By integrating out the underlying degrees of freedom of particles' coordinates, we can obtain a continuum, field-theoretic model. The system is described entirely by collective variables, e.g., the composition of the two species $m$ in block copolymers. 
For instance, the starting point of the SCFT is the soft, coarse-grained, particlebased model. Based on the Hubbard-Stratonovich transformation, the partition function of the particle-based model is rewritten as a field-theoretic model [48]. This field-theoretic model can be numerically solved by a saddle-point approximation which neglects the thermal fluctuations of collective variables. The advantage of this method is that it associates coarse-grained parameters with physically accessible variables. As shown in Fig. 1.3, the SCFT method qualitatively predicts the phase diagram of diblock copolymer melts. A detailed derivation of the SCFT method is presented in the Sec. 2.4.

\subsection{Outline}

This dissertation is mainly focusing on the non-equilibrium, self-assembly process of block copolymers. Especially, we study the kinetics of structure formation in symmetric diblock copolymer melts after a quench from a disordered, high-temperature state.

In the second chapter, we introduce a soft, particle-based, coarse-grained model which captures only relevant physical properties to bring about the self-assembly of block copolymers. This particle-based model preserves the basic description of block copolymers with a reduced number of degrees of freedom. We use two numerical approaches to investigate the self-assembly of block copolymers: The first approach is the particle-based simulation based on the SingleChain-in-Mean-Field (SCMF) algorithm. The second approach is the numerical SCFT method. To realize a large-scale simulation or calculation, we implement both algorithms with the GPU-acceleration technique (i.e., CUDA). For both approaches, we test the validity of the algorithm by comparing the results with analytical approaches.

In the third chapter, we develop techniques to derive the thermodynamic force in diblock copolymer systems. The thermodynamic force is the most important driving force in the course of structure formation. Due to the connectivity of the polymer chain, the thermodynamic force will be transmitted along the molecular backbone which is quantified by the Onsager coefficient. We devise a numerical scheme to calculate the Onsager coefficient with our particle-based simulations. One important application of the thermodynamic force is to derive 
the MFEP which provides the most fundamental information of the free-energy landscape for predicting the structure formation process.

In the fourth chapter, we start to systematically investigate the pattern formation process of symmetric diblock copolymers in a quasi-two-dimensional system with massive large-scale, particle-based simulations. After quenching the diblock copolymer melts from a highly disordered, melts, the block copolymer melts phase separate via a spinodal decomposition process. The ordering process is quantified by measuring the correlation length, the defects density, and the Euler characteristic of the morphology. The latter quantity measures the change of the topological structure. We find that the topological structures of the morphology are related to the free-energy basins of the free-energy landscape. We generalize three kinds of defects from a direct observation of the morphologies. We investigate the defect elimination process of these defects by means of the string method.

At the end of this thesis, we summaries important findings of our research and discuss future research. 


\section{Chapter 2}

\section{Model and Computational Techniques}

The aim of this chapter is to introduce the soft, particle-based, coarse-grained model to study static and dynamic properties of block copolymers. In the first section, we briefly summarize the physical background of block copolymer selfassembly. In the second section, we explicitly discuss the particle-based model, which is a minimal model that only captures relevant parameters to bring about the block copolymer self-assembly. In the end, we introduce the computational techniques, i.e., the self-consistent field theory and the single-chain-in-meanfield algorithm to investigate the minimal model.

\subsection{Phase separation and microphase separation}

A mixture which consists of more than two different chemical species may separate into several regions with different compositions. Although entropy always favors mixing, the weak repulsive force between unlike species can either promote or inhibit mixing. Whether the equilibrium state of a given mixture is homogeneous or phase separated is determined by the energy and entropy changes upon mixing.

For example, the mixture of binary homopolymer blends will separate into two phases in equilibrium below the critical temperature. This phenomenon 
is described by the Flory-Huggins theory. This theory successfully predicts the phase transition of the polymer mixture [49-52].

In a binary homopolymer blend, there are $n_{A} \mathrm{~A}$ polymers and $n_{B} \mathrm{~B}$ polymers in the melt. The polymerization of A polymer and B polymer is $N_{A}$ and $N_{B}$, respectively. In a lattice model with an incompressibility constraint, each monomer can only occupy one lattice site. The total number of lattice sites, $\Omega$ equals to $N_{A} n_{A}+N_{B} n_{B}$. Each lattice has $z$ neighboring lattices. The interaction between monomer $\alpha=\mathrm{A}(\mathrm{B})$ and monomer $\alpha^{\prime}=\mathrm{A}(\mathrm{B})$ is $\epsilon_{\alpha \alpha^{\prime}}$.

We use the volume fraction of A polymers, $\phi_{A}$ as the order parameter. The freeenergy difference of mixing of the binary homopolymer blend per lattice site is,

$$
\frac{f_{\mathrm{F}}\left(\phi_{A}\right)}{k_{\mathrm{B}} T}=\frac{1}{N_{A}} \phi_{A} \ln \phi_{A}+\frac{1}{N_{B}}\left(1-\phi_{A}\right) \ln \left(1-\phi_{A}\right)+\chi \phi_{A}\left(1-\phi_{A}\right) .
$$

$\chi \equiv \frac{z}{2 k_{\mathrm{B}} T}\left(2 \epsilon_{A B}-\epsilon_{A A}-\epsilon_{B B}\right)$ is defined as the Flory-Huggins parameter. This quantity is temperature dependent. Empirically, one often finds the relation,

$$
\chi(T) \approx A+\frac{B}{T}
$$

The term $A$ is referred as the "entropic part" of $\chi$ and the term $B / T$ is the "enthalpic part".

The free energy, $f_{\mathrm{F}}$ determines whether the system remains homogeneous or will phase separate. When $\chi=0$, the system favors mixing. There is only one minimum in free energy, $f_{\mathrm{F}}$, the system is homogeneous in equilibrium. For symmetric homopolymer blends with $N_{A}=N_{B}$ and $n_{A}=n_{B}$, there are two minima in the free energy, $f_{\mathrm{F}}: \phi_{A 1}$ and $\phi_{A 2}$. The critical point is at $\chi N_{A}=2$. If the volume fraction of the A polymer, $\phi_{A}$ is in the region where $\partial^{2} f_{\mathrm{F}} / \partial \phi_{A}^{2}<0$, the homogeneous mixture is unstable. The homopolymer blend will spontaneously phase separate into two phases with $\phi_{A 1}$ and $\phi_{A 2}$, respectively.

In diblock copolymer melts, due to the chemically bonded forces, the repulsive force between unlike segments cannot lead to a macroscopic phase separation. Instead one obtains the microphase separation, in which $\mathrm{A}(\mathrm{B})$-rich microdomains are formed. After the microphase separation, the small domains are not randomly distributed. These domains form periodical structures in the thermodynamical equilibrium state. 
To describe the microphase separation for block copolymer melts, theoretical approaches must take into account the long-range energies from the connectivity of the polymer chain. For a phenomenological approach, the long-range energies can be described by suitable approximations.

For example, the Ohta-Kawasaki free-energy functional has been successfully applied to investigate the structure formation of diblock copolymers in a quasitwo-dimensional system on surface patterns [53]. Suppose the polymerization of the block copolymer is $N$ which consists of A and B blocks with equal polymerization index, i.e., $N_{A}=N_{B}$. This model takes the difference of local volume fractions $m(\vec{r}, t)=\Phi_{A}(\vec{r}, t)-\Phi_{B}(\vec{r}, t)$ as the order parameter. The OhtaKawasaki free-energy functional is given by [54],

$$
\begin{aligned}
\frac{\mathcal{F}_{\mathrm{OK}}[m]}{n k_{\mathrm{B}} T}= & \frac{1}{V}\left\{\int \mathrm{d} \vec{r}\left[-\frac{\epsilon}{2} m^{2}+\frac{\mu}{4} m^{4}+\frac{K}{2}(\nabla m)^{2}\right]\right. \\
& \left.+\frac{D_{c}}{2} \int G\left(\vec{r}, \vec{r}^{\prime}\right)(m-\bar{m})(m-\bar{m}) \mathrm{d} \vec{r} \mathrm{~d} \vec{r}^{\prime}\right\} .
\end{aligned}
$$

$\epsilon$ and $\mu$ are phenomenological parameters which are from the phenomenological Ginzburg-Landau free energy [27]. $V$ is the volume of the system and $n$ is the total number polymers in the system. The parameter $K$ sets the square of the characteristic length scale and it is proportional to $R_{\mathrm{e} 0}^{2} \cdot \bar{m}$ is defined as the average of $m, \bar{m}=\frac{1}{V} \int \mathrm{d} \vec{r} m(\vec{r}, t)$. The chain connectivity is captured by adding the contribution with the Green function $G\left(\vec{r}, \vec{r}^{\prime}\right)$ to the phenomenological freeenergy functional. The Green function $G\left(\vec{r}, \vec{r}^{\prime}\right)$ satisfies,

$$
-\nabla^{2} G\left(\vec{r}, \overrightarrow{r^{\prime}}\right)=\delta\left(\vec{r}-\overrightarrow{r^{\prime}}\right)
$$

The coefficient $D_{c}$ depends on the polymerization index and the volume faction.

The biggest advantage of this continuum theory is that it can realize large time and length scale simulation compared to many particle-based simulations. However, this method is rather limited. For example, to evolve the order parameter, $m$, one usually employs a Cahn-Hilliard type dynamic equation in previous works [55]. In some occasions, such dynamic equation cannot describe the dynamics correctly [56]. Meanwhile, a continuum model which employs the density field as the order parameter is not appropriate when the polymer chain is strongly stretched [57]. 
In order to explicitly investigate structure formation process in block copolymer melts, we will introduce a particle-based model and numerical methods to solve this model in the following section.

\subsection{Soft, particle-based, coarse-grained model}

We use a coarse-grained model to investigate the self-assembly of block copolymers on a mesoscopic length scale. The advantage of a coarse-grained model is that it is computationally efficient. A coarse-grained model comprises only a few coarse-grained parameters that can be obtained by comparisons to experiments. Simultaneously, this model makes a direct connection to a field-theoretic description.

We adapt to a soft, computationally simple, top-down model [46], which captures only relevant interactions, i.e., the connectivity along the macromolecular backbone, the repulsive force between unlike species, and the limited compressibility of the dense polymer melts. We use the bonded interaction to describe the connectivity of polymer chain and the non-bonded interactions which give rise to the lateral two effects. This model is not limited to a specific block copolymer system. For simplicity, we only focus on the coarse-grained model that describes linear diblock copolymers in this section.

\subsubsection{Bonded interaction}

In a coarse-grained description, a polymer is discretized into $N$ interaction centers or coarse-grained segments. The position of the $s^{\text {th }}$ segment is denoted as $\vec{r}(s)$. We use a bead-spring model to describe the connectivity properties of the molecular backbone. The distance between neighboring segments is not fixed. The discretized bead-spring model gives a Gaussian probability distribution function for the mean-square segment-segment distance, $\left\langle\left[\vec{r}(s)-\vec{r}\left(s^{\prime}\right)\right]^{2}\right\rangle$.

The Hamiltonian of the bead-spring model for a single polymer can be written as,

$$
\frac{\hat{\mathcal{H}}_{b}}{k_{\mathrm{B}} T}=\sum_{<s, s^{\prime}>}^{N-1} \frac{3(N-1)}{2 R_{\mathrm{e} 0}{ }^{2}}\left[\vec{r}(s)-\vec{r}\left(s^{\prime}\right)\right]^{2} .
$$


$\left\langle s, s^{\prime}\right\rangle$ represents the set of interactions between segment $s$ and $s^{\prime}$, which determines the contour of the molecule. For example, the linear polymer chain comprises only the nearest connections between segments. The set $\left\langle s^{\prime}, s\right\rangle$ comprises $N-1$ interactions and the elements satisfy the relation $s^{\prime}=s+1 . R_{\mathrm{e} 0}{ }^{2}$ is the mean-square end-to-end distance of the ideal polymer chain, $R_{\mathrm{e} 0}{ }^{2}=$ $\left\langle[\vec{r}(N-1)-\vec{r}(0)]^{2}\right\rangle$, which is only subjected to $\mathcal{H}_{\mathrm{b}}$.

\subsubsection{Non-bonded interaction}

The bonded interactions mentioned in the above section give rise to the longrange energies, which are necessary to describe the microphase separation. To bring about the self-assembly of block copolymers, we also need to consider the non-bonded interactions, which gives rise to a near-incompressibility of the block copolymer melts and the weak repulsive interaction between different species.

The non-bonded interactions can be modeled by a minimal free-energy functional [46],

$$
\begin{aligned}
\frac{\hat{\mathcal{H}}_{\mathrm{nb}}}{k_{\mathrm{B}} T \sqrt{\mathcal{N}}}= & \frac{\kappa N}{2} \int \frac{\mathrm{d}^{3} \vec{r}}{R_{\mathrm{e} 0}^{3}}\left[\hat{\phi}_{A}(\vec{r})+\hat{\phi}_{B}(\vec{r})-1\right]^{2} \\
& -\frac{\chi N}{4} \int \frac{\mathrm{d}^{3} \vec{r}}{R_{\mathrm{e} 0}^{3}}\left[\hat{\phi}_{A}(\vec{r})-\hat{\phi}_{B}(\vec{r})\right]^{2} .
\end{aligned}
$$

To convert the coordinates of segments, $\vec{r}(s)$ to a collective density description, $\hat{\phi}_{\alpha}(\vec{r})$ of different species $\alpha=\mathrm{A}(\mathrm{B})$, we have the relations,

$$
\hat{\phi}_{\alpha}(\vec{r}) \equiv \frac{1}{\rho} \sum_{i=1}^{n} \sum_{s} \delta\left(\vec{r}-\vec{r}_{i}(s)\right) \gamma_{\alpha}(s) .
$$

The expression $\gamma_{\alpha}(s)=1$, if the segment $s$ of the block copolymer is the type $\alpha$, and $\gamma_{\alpha}(s)=0$ otherwise. $i$ labels the $i^{t h}$ polymer in the melts and $\rho=N n / V$ is the density of all segments. 
The Flory-Huggins parameter $\chi N$ is the thermodynamic incompatibility between species A and B. $\kappa N$ is the inverse isothermal compressibility that quantifies the strength of density fluctuations. The typical length scale of the coarsegrained model is set by a fraction of $R_{\mathrm{e} 0}$. In our simulation, we use the value $\kappa N=50$, which is sufficient to restrain the density fluctuations on a small length scale of a small fraction of $R_{\mathrm{e} 0}$.

The invariant degree of polymerization $\overline{\mathcal{N}} \equiv\left(\frac{\rho}{N} R_{\mathrm{e} 0}{ }^{3}\right)^{2}$ measures the degree of interdigitation of molecules. We use the value of $\overline{\mathcal{N}}=(128)^{2}$, which is a typical value in experiments. The long wavelength fluctuations become less important when $\overline{\mathcal{N}}$ increases. In the limit of $\overline{\mathcal{N}} \rightarrow \infty$, many properties of this minimal model (e.g., the equilibrium phase behavior and the chain conformation in spatially modulated phase) can be accurately estimated by mean-field methods (e.g., SCFT). $\overline{\mathcal{N}}$ is a coarse-grained parameter, which is independent of the discretization of the chain contour. To quantitatively describe a physical system, it is important to understand how thermal fluctuations modify physical properties. For example, the critical point of the symmetric diblock copolymer melts is shifted in the particle-based simulation due to thermal fluctuations. The second-order transition of the mean-field prediction is also altered to the fluctuation-induced first-order transition [58].

In the following thesis, we will introduce the SCFT method and the particlebased, Single-Chain-in-Mean-Field (SCMF) algorithm. The SCMF algorithm goes beyond the mean-field approximation. By tuning the coarse-grained parameter $\overline{\mathcal{N}}$, we can mimic the SCFT results in the particle-based simulation. We will discuss the impact of fluctuations and compare the two methods in different systems.

\subsection{Particle-based simulation}

In this section, we will introduce the particle-based, SCMF algorithm. This method is based on the smart Monte Carlo algorithm which mimics the singlechain dynamics, e.g., the Rouse dynamics.

The SCMF algorithm retains the computational advantage of a Monte Carlo method and accurately describes long wavelength fluctuations. Meanwhile, this method allows an efficient implementation of a parallel computation. 
In the following, we will introduce the SCMF algorithm and discuss some results from particle-based simulations.

\subsubsection{Rouse dynamics}

A flexible polymer chain in solution or melts has a large number of degrees of freedom. The motion of a molecule can be observed by using the fluorescence microscopy [59]. In a coarse-grained description, the dynamics of a single polymer chain can be described with the Rouse dynamics. The effective segment along the polymer chain is considered as a spherical object moving in a solvent. It will experience a viscous force which is proportional to its velocity, and the direction is opposite to its velocity. The effective segments will also experience a random or stochastic force.

Besides the random force and the viscous force, the segment of a polymer will also experience forces by the bonded interactions and the non-bonded interactions which are introduced in section 2.2.

In this section, we only discuss the dynamics of a linear polymer chain in the melts which only subject to the bonded force. The Hamiltonian of the linear chain with a short-range nearest neighbor interaction was first proposed by Prince E. Rouse in 1953 [60] and is described in Eq. 2.5.

In Rouse dynamics, the equation of motion of segments is called the Langevin equation [61]. Surrounding polymers provide only a fluctuating background, which produces a viscous friction and a thermal noise.

The Langevin equations of segments along the molecular backbone are,

$$
\begin{aligned}
\xi_{0} \frac{\mathrm{d} \vec{r}_{i}(s)}{\mathrm{d} t} & =-\frac{3 N}{R_{\mathrm{e} 0}^{2}}\left[2 \vec{r}_{i}(s)-\vec{r}_{i}(s-1)-\vec{r}_{i}(s+1)\right]+\vec{\eta}_{i}(s, t) \\
\xi_{0} \frac{\mathrm{d} \vec{r}_{i}(0)}{\mathrm{d} t} & =-\frac{3 N}{R_{\mathrm{e} 0}^{2}}\left[\vec{r}_{i}(0)-\vec{r}_{i}(1)\right]+\vec{\eta}_{i}(0, t) \\
\xi_{0} \frac{\mathrm{d} \vec{r}_{i}(N-1)}{\mathrm{d} t} & =-\frac{3 N}{R_{\mathrm{e} 0}^{2}}\left[\vec{r}_{i}(N-1)-\vec{r}_{i}(N-2)\right]+\vec{\eta}_{i}(N-1, t)
\end{aligned}
$$


$\vec{\eta}_{i}(s, t)$ is a random number with zero mean and unit variance which satisfies,

$$
\begin{aligned}
\left\langle\vec{\eta}_{i}(s, t)\right\rangle & =0 \\
\left\langle\eta_{i}(s, t) \eta_{i^{\prime}}\left(s^{\prime}, t^{\prime}\right)\right\rangle & =2 \xi_{0} k_{\mathrm{B}} T \delta_{s s^{\prime}} \delta_{t t^{\prime}} \delta i i^{\prime} .
\end{aligned}
$$

$\xi_{0}$ is the segmental friction in the coarse-grained model. In contrast to the Lennard-Jones bead-spring model, segmental interactions in our minimal model are very soft can hardly influence the friction. Thus, in our coarse-grained, this parameter is an input constant. As a consequence, polymers will change their conformation with a local unconstrained move. An unconstrained move refers to a kind of movement of segments which may violate the topological constraint of the molecules.

The self-diffusion constant in a homogeneous melt is $D_{\text {Rouse }}$ and the center-ofmass $\mathbf{R}_{G}$ of the molecule is defined as,

$$
\mathbf{R}_{G} \equiv \frac{1}{N} \sum_{s=0 \ldots N-1} \vec{r}(s) .
$$

The mean-square center-of-mass displacement $g_{3}(t)$ obeys the linear relation,

$$
g_{3}(t)=\left\langle\left[\mathbf{R}_{G}(t)-\mathbf{R}_{G}(0)\right]^{2}\right\rangle=6 D_{\text {Rouse }} t .
$$

Within the Rouse model, the self-diffusion constant $D_{\text {Rouse }}$ can be calculated as,

$$
D_{\text {Rouse }}=\lim _{t \rightarrow \infty} \frac{1}{6 t}\left\langle\left(\mathbf{R}_{G}(t)-\mathbf{R}_{G}(0)\right)^{2}\right\rangle=\frac{k_{\mathrm{B}} T}{N \xi_{0}} .
$$

The Rouse dynamics correctly describes the long-time diffusion of a polymer chain in a melt with a short chain length. In the following thesis, we will use the characteristic time $\tau$ which is the longest relaxation time of a polymer chain in the homogeneous phase as the unit time. $\tau$ is the time to completely equilibrate a chain conformation. It is defined by using the time that the mean-square center-of-mass displacement of $g_{3}(t)$ diffuses a distance of $R_{\mathrm{e} 0}^{2}$,

$$
\tau \equiv \frac{R_{\mathrm{e} 0}^{2}}{D_{\text {Rouse }}} .
$$


$R_{\mathrm{e} 0}{ }^{2}$ is the mean-square end-to-end distance of the ideal polymer in the melt.

We use the time scale $\tau$ to characterize our molecular simulation, because the conformation of a polymer chain is completely renewed once the chain has diffused its own end-to-end distance.

The advantage of the Rouse model is that it preserves the long-time diffusion properties of the polymer chain in melts [56].

\subsubsection{Smart Monte Carlo algorithm}

The SCMF algorithm is based on the Monte Carlo sampling method. Configurations are generated according to the Boltzmann weight of the system's Hamiltonian. The Monte Carlo procedure of Metropolis et al. is widely applied to estimate the equilibrium properties of a polymer system [62].

Instead of randomly selecting a trial move or a new configuration, one can improve the Monte Carlo algorithm by enforcing the trial move to mimic the Rouse dynamics. Based on this idea, the smart Monte Carlo method is proposed by Rossky and et al. [63]. The trial moves of segments are chosen in accordance with a Rouse dynamics rather than at random.

Compared with the standard Metropolis procedure of the Monte Carlo sampling method, there are two main advantages of this approach: (i) The smart Monte-Carlo produces more rapid convergences. The acceptance rate is increased by imposing the instantaneous force on the segments. (ii) The smart Monte Carlo method preserves the single-chain dynamics which mimics a realistic dynamics.

In the smart Monte Carlo method, one randomly selects a segment along the polymer chain with equal possibilities for all segments. The equation of motion of the selected segment is governed by the Langevin equations 2.8. We use a simple Euler scheme to mimic the Brownian dynamics of segments,

$$
{\overrightarrow{r^{\prime}}}_{i}(s)=\vec{r}_{i}(s)+\frac{\triangle t}{\xi_{0}} \mathbf{F}_{i}(s)+\frac{\sigma_{0}}{\xi_{0}} \sqrt{\triangle t} \eta_{i}
$$

$\sigma_{0}^{2}=2 \xi_{0} k_{\mathrm{B}} T$ and the new trial position of the segment ${\overrightarrow{r^{\prime}}}_{i}(s)$ is to be tested by the Monte Carlo criterion. The force exerted onto the effective segment $\mathbf{F}_{i}(s)$ are the 
bonded interactions which keep the contour connectivity of the polymer chain and the non-bonded interactions. In our numerical implement, we neglect the latter contribution to the polymer dynamics. Because the contribution from the non-bonded interactions is magnitude smaller than the contribution from the bonded interactions. The more repeated units are lumped into the coarsegrained segments, the weaker the segmental interactions.

We define $\Delta A$ and $\Delta R$ to simplify the notation in Eq. 2.15,

$$
\begin{aligned}
\Delta A & =\Delta t / \xi_{0}, \\
\Delta R & =\sqrt{2 k_{\mathrm{B}} T \Delta A} \eta_{i}(s, t) .
\end{aligned}
$$

The equation of motion of segments is modified as,

$$
{\overrightarrow{r^{\prime}}}_{i}(s)=\vec{r}_{i}(s)+\Delta A \mathbf{F}_{i}(s)+\Delta R
$$

The probability to propose the trial move that transfers the segment from the position $\vec{r}_{i}(s)$ to the new position $\vec{r}_{i}^{\prime}(s)$ is,

$$
T\left[\vec{r}_{i}(s) \rightarrow{\overrightarrow{r^{\prime}}}_{i}(s)\right]=\frac{1}{(4 \pi \Delta A)^{-3 / 2}} \exp \left(\frac{\Delta R^{2}}{4 k_{\mathrm{B}} T \Delta A}\right)
$$

We define $A\left[\vec{r}_{i}(s) \rightarrow \vec{r}_{i}(s)\right]$ as the probability of a segment that is accepted in a trial move from $\vec{r}_{i}(s)$ to ${\overrightarrow{r^{\prime}}}_{i}(s)$. The detailed balance equation is established as,

$$
\frac{1}{N} A\left[\vec{r}_{i}(s) \rightarrow{\overrightarrow{r^{\prime}}}_{i}(s)\right] T\left[\vec{r}_{i}(s) \rightarrow{\overrightarrow{r^{\prime}}}_{i}(s)\right]=\frac{1}{N} A\left[\vec{r}_{i}(s) \rightarrow \vec{r}_{i}(s)\right] T\left[{\overrightarrow{r^{\prime}}}_{i}(s) \rightarrow \vec{r}_{i}(s)\right]
$$

According to the detailed balance condition and the Metropolis treatment, the acceptance criteria of this trial movement is,

$$
\begin{aligned}
A\left[\vec{r}_{i}(s) \rightarrow{\overrightarrow{r^{\prime}}}_{i}(s)\right] & =\min \left\{1, \exp \left[-\frac{1}{k_{\mathrm{B}} T}\left[E\left({\overrightarrow{r^{\prime}}}_{i}(s)\right)-E\left(\vec{r}_{i}(s)\right.\right.\right.\right. \\
& +\left(\frac{\mathbf{F}\left({\overrightarrow{r^{\prime}}}_{i}(s)\right)+\mathbf{F}\left(\vec{r}_{i}(s)\right)}{2}\right)\left({\overrightarrow{r^{\prime}}}_{i}(s)-\vec{r}_{i}(s)\right) \\
& \left.\left.+\frac{\Delta A}{4}\left(\mathbf{F}\left({\overrightarrow{r^{\prime}}}_{i}(s)\right)^{2}-\mathbf{F}\left(\vec{r}_{i}(s)\right)^{2}\right)\right]\right\}
\end{aligned}
$$




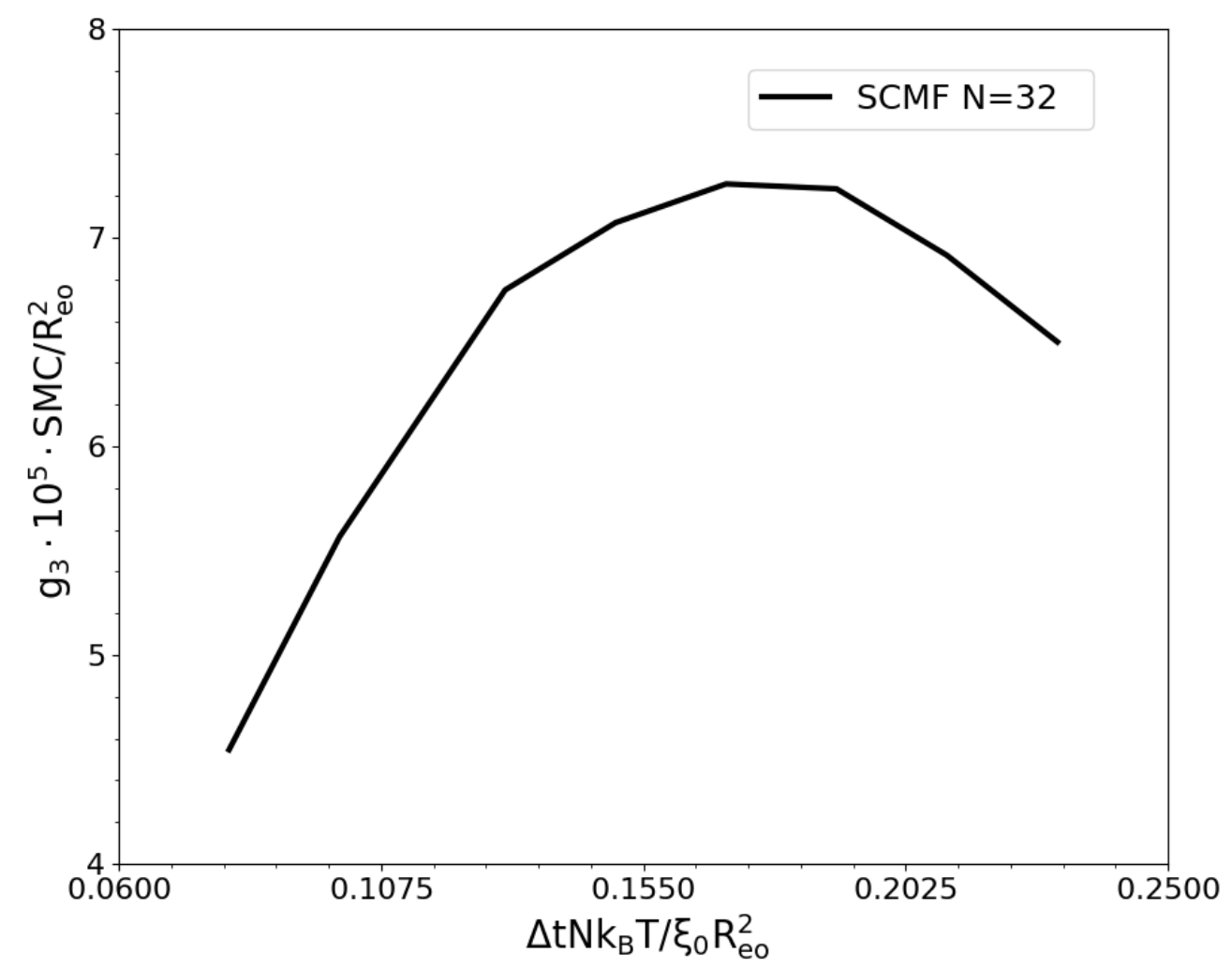

FIGURE 2.1: Mean-square center-of-mass displacement, $g_{3}$, per SMC step as a function of the time step length, $\Delta t . g_{3}$ is measured after $10^{5} \mathrm{SMCs}$ in the particle-based simulation.

where the energy $E=\mathcal{H}_{b}+\mathcal{H}_{n b}$ is the total energy of the system which is subjected to the bonded and non-bonded interactions.

We define the smart Monte Carlo step (SMC) as the average time that all segments have the chance to move once.

To implement the smart Monte Carlo method efficiently, one has to determine a proper time step length, $\Delta t$ in the equation of motion of the segments in Eq. 2.15. In the limit of a very small time step $\Delta t$, all the trial moves will be accepted. The particle system automatically mimics the Rouse dynamics. If we increase the value of $\Delta t$, the acceptance rate of the trial move will decrease and also the dynamics will deviate from the Rouse dynamics.

The proper time step length, $\Delta t$ is obtained by measuring the mean-square center-of-mass displacement, $g_{3}(t)$. As shown in the Fig. 2.1, $g_{3}(t)$ is plotted 
as a function of $\Delta t$ in one SMC. It has a maximum at $\Delta t_{0}[64]$,

$$
\Delta t_{0}=\frac{0.17 R_{\mathrm{e} 0}^{2} \xi_{0} N}{N^{2} k_{\mathrm{B}} T}
$$

In this dissertation, we will use $\Delta t_{0}$ as the time step in the simulation. Because the diffusion of the polymer reaches the maximum at $\Delta t_{0}$.

\subsubsection{Single-Chain-in-Mean-Field (SCMF) algorithm}

The idea of the SCMF algorithm origins from the SCFT method. The nonbonded interactions between polymers are decoupled and replaced by external fields. In the SCMF algorithm, polymers are regarded as isolated molecules in the environment of external fields $\hat{w}_{A}$ and $\hat{w}_{B}$. These fields are kept constant in a short, predetermined simulation time and are frequently updated. This quasi-instantaneous field approximation significantly enhances the simulation efficiency and makes it possible to implement the computational algorithm on a parallel high-performance computer. The SCMF method retains the computational advantages of the SCFT method and more importantly, the SCMF algorithm includes thermal fluctuations, which are ignored in the SCFT method.

Before introducing the SCMF algorithm, we need to devise strategies to regularize the delta function in the microscopic density $\hat{\phi}_{A}$ and $\hat{\phi}_{B}$ to adapt to a computer simulation. Generally, there are two approaches: One is the grid-based scheme and the other one is the weighting function scheme.

In the grid-based scheme, the volume is discretized by cubic cells of linear length, $\Delta L$. The cell index is identified by the index c. The density $\hat{\phi}_{\alpha}$ of the segments $\alpha=A$ (or $B$ ) on the cell $\mathbf{c}$ is,

$$
\hat{\phi}_{\alpha}(\mathbf{c})=\int_{\triangle V} \frac{d^{3} \vec{r}}{\triangle V} \hat{\phi}_{\alpha}(\vec{r})=\int \frac{\mathrm{d}^{3} \vec{r}}{\triangle V} \hat{\phi}_{\alpha}(\vec{r}) \Pi(\vec{r}, \mathbf{c}) .
$$

The weight function $\Pi(\vec{r}, \mathbf{c})$ is normalized and satisfy,

$$
\begin{aligned}
\sum_{\mathbf{c}} \Pi(\mathbf{c}, \vec{r}) & =1 \\
\int \mathrm{d}^{3} \vec{r} \Pi(\mathbf{c}, \vec{r}) & =\Delta L^{3}
\end{aligned}
$$


The zeroth-order assignment function, $\Pi_{0}$ associates the segments onto the nearest grid point,

$$
\Pi_{0}(\mathbf{c}, \vec{r})= \begin{cases}1 & \text { if }\left|\vec{r}_{\alpha}-\mathbf{c}_{\alpha}\right|<\Delta L / 2 \\ 0 & \text { else. }\end{cases}
$$

where $\alpha$ is in $\{x, y, z\}$.

The first-order assignment function is defined by,

$$
\Pi_{1}(\mathbf{c}, \vec{r})= \begin{cases}\prod_{\alpha=x, y, z}\left(1-\frac{\left|\vec{r}_{\alpha}-\mathbf{c}_{\alpha}\right|}{\Delta L}\right) & \text { if }\left|\vec{r}_{\alpha}-\mathbf{c}_{\alpha}\right|<\Delta L / 2 \\ 0 & \text { else. }\end{cases}
$$

The advantage of the zeroth-assignment function is that it is computationally simple and is easy to implement. The disadvantage of this scheme is that it produces discontinuous changes in the density profile when a particle straddles a cell boundary. For instance, the zero-assignment function gives rise to a discontinuous non-bonded force, $\frac{\partial \mathcal{H}_{\mathrm{nb}}}{\partial \vec{r}_{i}(s)}$ that acts on the $s^{\text {th }}$ segment of polymer $i$.

Instead of assigning the particle coordinates to grid points with the grid-based scheme, it is also possible to represent the delta function of the density $\hat{\phi}_{\alpha}$ as a limit of a continuous, weight function, $\omega[65,66]$.

The local, microscopic density, $\hat{\phi}_{\alpha}(\vec{r})$ represented by the weight function $\omega$ is obtained according to,

$$
\hat{\phi}_{\alpha, \omega}(\vec{r})=\frac{1}{\Delta L^{3}} \int \mathrm{d}^{3} \overrightarrow{r^{\prime}} \omega\left(\left|\vec{r}-\overrightarrow{r^{\prime}}\right|\right) \hat{\phi}_{\alpha}\left(\overrightarrow{r^{\prime}}\right)
$$

The weight function is a continuous function and is normalized as,

$$
\int \frac{\mathrm{d}^{3} \vec{r}}{\Delta L^{3}} \omega(|\vec{r}|)=\Delta L^{3}
$$

where $\Delta L^{3}$ represent the volume which is important to characterize the original $\delta$-function. 
One possible form of the weight function is [66],

$$
\omega=\left(\frac{3}{2 \pi}\right)^{3 / 2}\left(\frac{\Delta L}{\sigma}\right)^{3} \exp \left(-\frac{3 \vec{r}^{2}}{2 \sigma^{2}}\right)
$$

The advantage of the weight function scheme is that it is convenient to compute the pressure without special techniques due to the translational invariance and isotropy of the pairwise potential [67]. The major disadvantage is the computational expense of calculating the pairwise interactions.

In the grid-based approach, the energy contribution of a segment caused by the non-bonded interaction can be computed from the knowledge of the gridbased density. Thus the grid-based technique offers a significant computational advantage for dense polymer systems compared to the weight function scheme. In this thesis, we use the grid-based scheme with the zeroth-assignment function.

The Hamiltonian of non-bonded interactions in the discretized form is written as,

$$
\begin{aligned}
\frac{\hat{\mathcal{H}}_{n b}}{k_{\mathrm{B}} T \sqrt{\overline{\mathcal{N}}}}= & \frac{\kappa N}{2} \sum_{\mathbf{c}} \frac{\Delta L^{3}}{R_{\mathrm{e} 0}{ }^{3}}\left[\hat{\phi}_{A}(\mathbf{c})+\hat{\phi}_{B}(\mathbf{c})-1\right]^{2} \\
& -\frac{\chi N}{4} \sum_{\mathbf{c}} \frac{\Delta L^{3}}{R_{\mathrm{e} 0}{ }^{3}}\left[\hat{\phi}_{A}(\mathbf{c})-\hat{\phi}_{B}(\mathbf{c})\right]^{2}
\end{aligned}
$$

We use the SCMF algorithm to accelerate the particle-based simulation. The non-bonded interactions between segments in the SCMF method are decoupled by introducing external fields $\hat{w}_{A}(\mathbf{c})$ and $\hat{w}_{B}(\mathbf{c})$.

The external fields $\hat{w}_{A}(\mathbf{c})$ are the derivatives of the Hamiltonian to the density of $A$ segments, 


$$
\begin{aligned}
\hat{w}_{A} & =\frac{R_{\mathrm{e} 0}{ }^{3}}{k_{\mathrm{B}} T \sqrt{\overline{\mathcal{N}} \Delta L^{3}}} \frac{\delta \hat{\mathcal{H}}_{n b}}{\delta \hat{\phi}_{A}(\mathbf{c})} \\
& =\kappa N\left[\hat{\phi}_{A}(\mathbf{c})+\hat{\phi}_{B}(\mathbf{c})-1\right]-\frac{\chi N}{2}\left[\hat{\phi}_{A}(\mathbf{c})-\hat{\phi}_{B}(\mathbf{c})\right] \\
\hat{w}_{B} & =\frac{R_{\mathrm{e} 0}{ }^{3}}{k_{\mathrm{B}} T \sqrt{\overline{\mathcal{N}} \Delta L^{3}}} \frac{\delta \hat{\mathcal{H}}_{n b}}{\delta \hat{\phi}_{B}(\mathbf{c})} \\
& =\kappa N\left[\hat{\phi}_{A}(\mathbf{c})+\hat{\phi}_{B}(\mathbf{c})-1\right]-\frac{\chi N}{2}\left[\hat{\phi}_{B}(\mathbf{c})-\hat{\phi}_{A}(\mathbf{c})\right]
\end{aligned}
$$

The non-bonded Hamiltonian in Eq. 2.6 can be approximated by,

$$
\frac{\hat{\mathcal{H}}_{\mathrm{nb}}}{k_{\mathrm{B}} T \sqrt{\overline{\mathcal{N}}}} \approx \sum_{\mathbf{c}}\left[\sum_{\alpha} \hat{w}_{\alpha}(\mathbf{c}) \hat{\phi}_{\alpha}(\mathbf{c})\right] \frac{\Delta L^{3}}{R_{\mathrm{e} 0}{ }^{3}} .
$$

The quasi-instantaneous fields are computed from the instantaneous densities according to Eq. 2.32. During the short time between the field updates, the molecules independently evolve in the temporarily constant fields.

The quality of the quasi-instantaneous field approximation is controlled by a parameter $\epsilon$ which is given by,

$$
\epsilon=\frac{V}{n N^{2} \Delta V}=\frac{1}{N^{2} \sqrt{\overline{\mathcal{N}}}}\left(\frac{R_{\mathrm{e} 0}}{\Delta L}\right)^{3} .
$$

This parameter is obtained by comparing the energy change with the exact MD simulations [68]. In the limit where $\epsilon \ll 1 / \max (\kappa N, \chi N)$, the updating external fields after every Monte Carlo step with the SCMF algorithm is quantitatively accurate.

From Eq. 2.35, the precision of the SCMF algorithm can be controlled by increasing the chain discretization, $N$ or decreasing the spatial resolution, $\Delta L$ without changing the physics of the system.

The SCMF algorithm is in analogy to the reversible reference system propagator algorithm (RESPA) in the MD simulation [69, 70]. This method uses a similar idea to numerically analyze high-frequency oscillators interacting with low-frequency baths [69]. It is also a multiple time step method that allows one to use a time step appropriate for the heavy particles. There is a separation of 
the time scale between the large and the small particles. In block copolymers, the weak non-bonded interactions give rise to collective processes, which often occur on time scales that exceed the relaxation time of a single molecule. A multiple time step method like SCMF is particularly suitable to describe "slow" collective phenomena.

The advantage of this particle-based algorithm is that the collective kinetics of the densities and the underlying dynamics of the individual macromolecular conformation are combined. We mimic the single-chain dynamics by the smart Monte Carlo scheme for a local unconstrained movement. In each Smart MonteCarlo step, every segment tries a displacement once on average.

Another important advantage of the SCMF algorithm is that the decoupling scheme of interactions between polymers allows a large-scale parallel computation. In the next subsection, we will introduce the implementation of the SCMF algorithm and compare the performance of the algorithm on CPUs and GPUs.

\subsubsection{Performance and workflow}

We implement the SCMF algorithm by using the MPI and the CUDA techniques for the hybrid parallelization approach. The SCMF algorithm is implemented on GPUs, we use the NCCL 1.0 library to optimize the communication between GPUs to achieve high bandwidth over PCIe [71]. The MPI parallelization is used to communicate data between computer nodes.

We demonstrate the implementation of the SCMF algorithm in a flowchart in the Fig. 2.2. We distribute the coordinates of polymers evenly on each GPU processors. Meanwhile each GPU processors stores a replica of the density fields, $\phi_{\alpha}$ and external potentials, $w_{\alpha}$.

As shown in Fig. 2.2, we first initialize the coordinates of the polymers. On each GPU processor, we can only calculate the density profiles $\phi_{\alpha}^{\prime}$ which are generated by a subset of polymers on each GPU by the Eq. 2.23. We use the MPI and NCCL library to gather the densities $\phi_{\alpha}^{\prime}$ in each GPU processor to generate the real distribution of the segments, $\phi_{\alpha}$. After obtaining the density $\phi_{\alpha}$, we broadcast the density back to each GPU processors. Next step, we generate the external fields, $w_{\alpha}$ with the density $\phi_{\alpha}$ by Eq. 2.32 . 


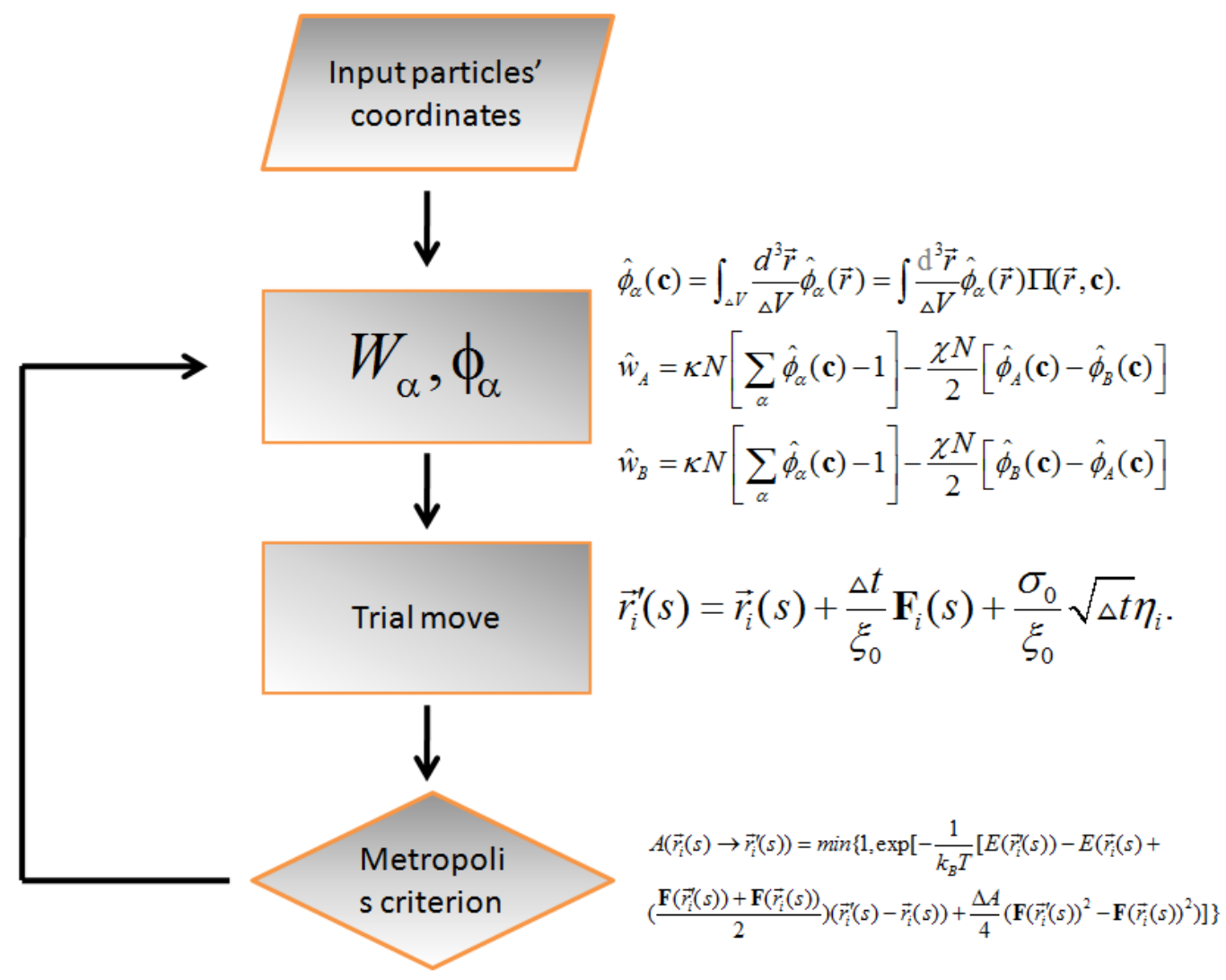

FIGURE 2.2: The flowchart of the SCMF algorithm.

At the third step, we loop over all the polymers and give every segment a chance to move once. The trial moves of the segments are generated by the Eq. 2.18. The new coordinates of the polymers are accepted or rejected according to the Monte Carlo criteria in Eq. 2.21. The total energy in the Monte Carlo criteria includes contributions both from the bonded interactions and the nonbonded interactions.

From above demonstrations of the SCMF algorithm, the number of the polymers, determines the speed of calculations on each GPU processors. The communication between processors are transferring the density fields, so the number of the discretized grid points $\mathrm{c}$ limits the efficiency of the parallel computation.

To test the SCMF algorithm on GPUs, we simulate symmetric diblock copolymers in a quasi-two-dimensional system. The thickness of the system is $1 R_{\mathrm{e} 0}$ and the size of the system along the other two directions are equally set as $L$. We keep the invariant degree of polymerization $\sqrt{\mathcal{N}}=128$ as a constant with a polymer chain discretization, $N=32$. 


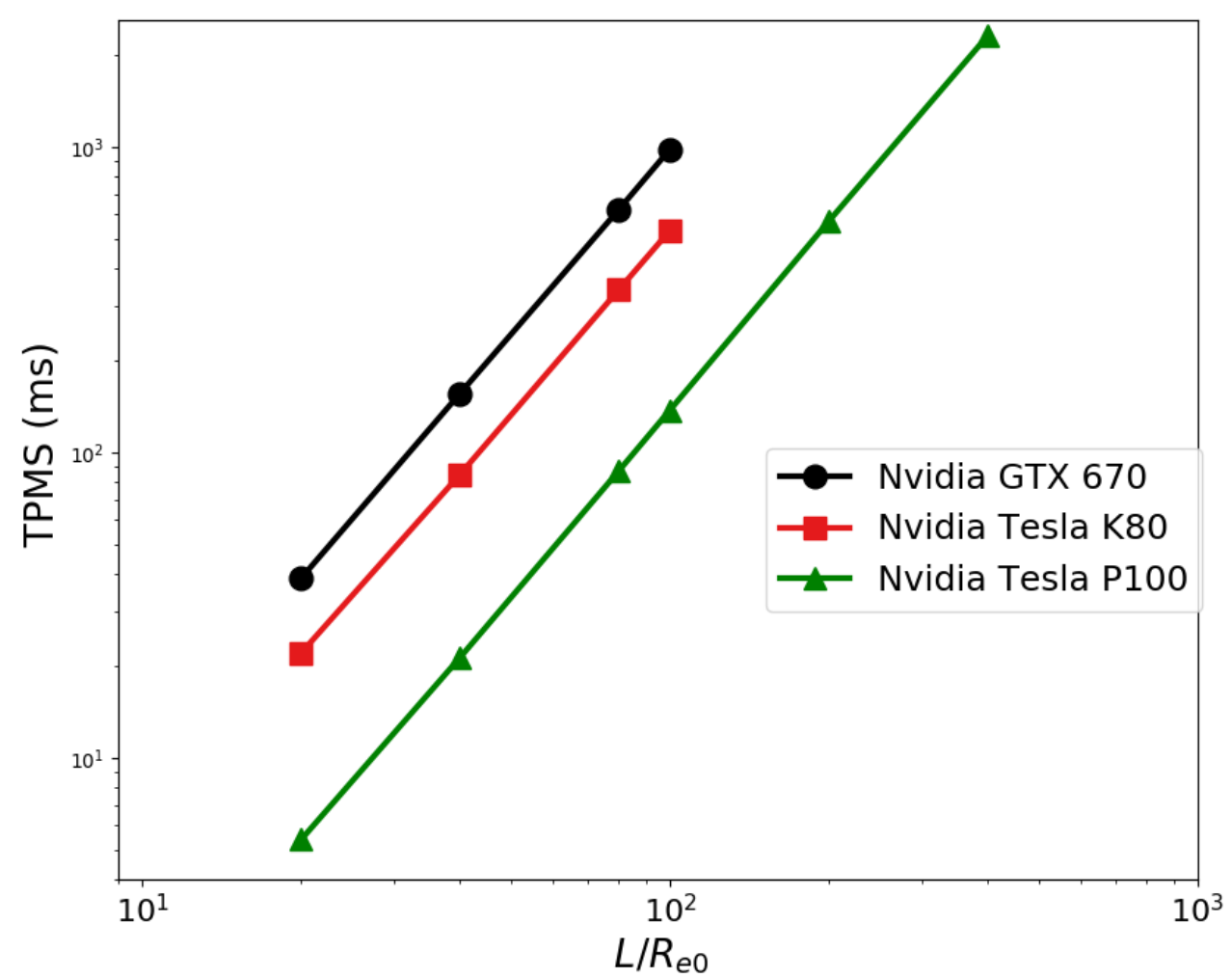

FIGURE 2.3: The performance of SCMF is tested on different GPUs, i.e., the GTX 670, Tesla P100 and K80. The invariant degree of polymerization, $\sqrt{\overline{\mathcal{N}}}=$ 128 is kept as a constant with a polymer chain discretization, $N=32$.

We test the performance of the SCMF algorithm on various graphics cards, i.e., the GTX 670, Tesla P100 and Tesla K80. We measure the simulation time per smart Monte Carlo step (TPMS) as a function of the system size $L$.

The results are presented in the following table 2.1 and the Fig. 2.3. $n$ is the total number of polymers in the system. The TPMS increases linearly with the system size, $L$ on different GPUs.

As a comparison, the single thread program on CPU is also tested on a system size $L=20 R_{\mathrm{e} 0}$. On the Core (TM) i3-3240 CPU @ 3.40GHz, it takes approximately $1200 \mathrm{~ms}$ for each SMC step.

We also test the performance of the SCMF algorithm on multiple GPUs. The results are shown in table 2.2 . 
TABLE 2.1: Performance of the SCMF algorithm on a single GPU core. The table measures the average time per SMC (TPMS) in unit of $m s$. The polymerization, $N$ is 32 .

\begin{tabular}{lllll}
\hline$L / R_{\mathrm{e} 0}$ & $n$ & Tesla P100 & Tesla K80 & GTX 670 \\
\hline 20 & $5.12 \times 10^{4}$ & 5.4028 & 22.05 & 38.7294 \\
40 & $2.048 \times 10^{5}$ & 21.2953 & 84.66 & 155.862 \\
100 & $1.28 \times 10^{6}$ & 137.497 & 532.70 & 982.311 \\
200 & $5.12 \times 10^{6}$ & 570.94 & - & - \\
400 & $2.048 \times 10^{7}$ & 2308.02 & - & - \\
\hline
\end{tabular}

TABLE 2.2: Performance of the SCMF algorithm on multiple GPUs Tesla K80 on a quasi-two-dimensional system with $L=100 R_{\mathrm{e} 0}$ with a polymerization, $N=32$.

\begin{tabular}{lll}
\hline Cores & TPIS (ms) & Speedup \\
\hline 1 & 522.57 & 1 \\
2 & 273.82 & 1.9 \\
4 & 149.42 & 3.5 \\
\hline
\end{tabular}

\subsubsection{A comparison of the SCMF algorithm with analytical re- sults}

In this subsection, we will compare the numerical results of the SCMF algorithm to the analytical results.

\subsubsection{Mean-square center-of-mass displacement in isotropic, homogeneous system}

In this subsection, we will study the single-chain dynamics generated by the SCMF algorithm. According to the Rouse dynamics, the mean-square, centerof-mass displacement, $g_{3}(t)$ in a homogeneous melt is given by the Eq. 2.12.

By the SCMF algorithm, we implement the particle-based simulation in a homogeneous phase of polymer melts. In Fig. 2.4, we plot the $g_{3}(t)$ as a function of the simulation time $t$. As predicted, $g_{3}(t)$ increases linearly with the simulation time $t$ at a large time scale. The dynamics of the single chain is isotropic in all directions. In Fig. 2.4, the curve $g_{3}^{x}, g_{3}^{y}$ and $g_{3}^{z}$ are overlap. 


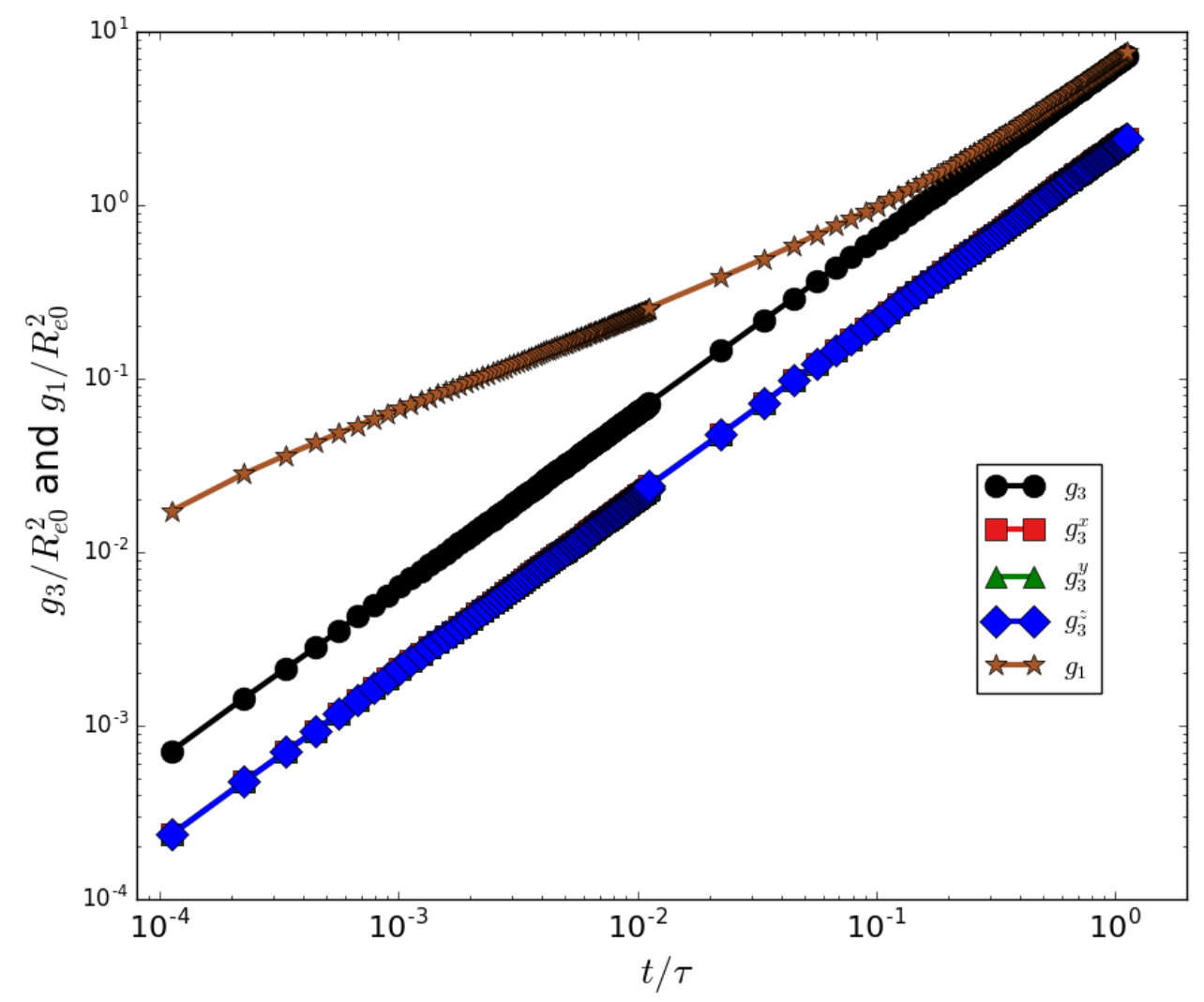

FIGURE 2.4: Mean-square, center-of-mass displacement, $g_{3}$ and mean-square displacement of segments, $g_{1}$ as a function of simulation time $t$.

In the Rouse model, the relaxation time, $\tau$ to equilibrate the polymer chain conformation is predicted as,

$$
\tau=\frac{R_{\mathrm{e} 0}^{2}}{D_{\text {Rouse }}}
$$

In the SCMF algorithm, the time step is determined as $\Delta t=0.17 \xi R_{\mathrm{e} 0}{ }^{2} / N k_{\mathrm{B}} T$. The self-diffusion constant, $D_{\text {scmf }}$ by the SCMF algorithm is calculated as $D_{\text {scmf }} \approx$ $0.0001123 R_{\mathrm{e} 0}{ }^{2} / \mathrm{SMC}$. It takes approximately $\tau=8900 \mathrm{SMC}$ to fully equilibrium the chain conformation. In this thesis, we use the time $\tau$ as the unit time.

We also present the mean-square displacements of all segments, $g_{1}(t)$ which is defined as,

$$
g_{1}(t)=\frac{1}{N} \sum_{s=0}^{N-1}\left\langle(\vec{r}(s, t)-\vec{r}(s, t=0))^{2}\right\rangle .
$$


In the Rouse model, the mean-square displacement of all segments, $g_{1}(t)$ is predicted as [56],

$$
\frac{g_{1}(t)}{R_{\mathrm{e} 0}{ }^{2}}=\frac{6 t}{\tau}+\frac{1}{N} \sum_{s} \sum_{p}\left(\frac{\cos \left(\frac{\pi p(s+0.5)}{N}\right)}{N \sin \left(\frac{\pi p}{2 N}\right)}\right)^{2} \times\left(1-e^{-12 N^{2} \sin (\pi p / 2 N) t / \tau}\right)
$$

The mean-square displacement of segments, $g_{1}(t)$ can be distinguished by three regions,

$$
\frac{g_{1}(t)}{R_{\mathrm{e} 0}{ }^{2}}= \begin{cases}\frac{6 N t}{\tau} & , t<\frac{\tau}{3 \pi N^{2}} \\ 2 \sqrt{\frac{3 t}{\pi \tau}} & , \frac{\tau}{3 \pi N^{2}}<t<\frac{\tau}{3 \pi} \\ \frac{6 t}{\tau} & , \frac{\tau}{3 \pi}<t .\end{cases}
$$

$\tau_{\mathrm{R}}$ is the Rouse time of the SCMF algorithm which is defined as $\tau_{\mathrm{R}} \equiv \tau / 3 \pi^{2}$.

The predictions of $g_{1}$ and $g_{3}$ obtained by the Rouse model agree with the particlebased, SCMF algorithm simulation. As shown in Fig. 2.4, $g_{3}$ and $g_{1}$ approximately collapse at $t>0.1 \tau$.

\subsubsection{Expansion of an isolated polymer chain}

In a polymer melt or solution, the spatial extent of a polymer chain may expand. The length scale of a polymer chain is characterized by the mean-square, endto-end distance, $\left\langle R_{\mathrm{e}}^{2}\right\rangle$, which is defined by,

$$
R_{\mathrm{e}}^{2}=\left\langle[\vec{r}(N)-\vec{r}(0)]^{2}\right\rangle \propto N^{2 \nu}
$$

$N$ is the polymerization of the polymer chain. The mean-square, end-to-end distance, $R_{\mathrm{e}}^{2}$ is proportional to $N^{2 \nu}$.

For ideal chains without non-bonded interactions, the simplified idealization model can be replaced by a random walk on a periodic lattice. Numerical studies on this ideal model show that $\nu$ depends only on the dimensionality of the system. The growth exponent $\nu$ can be easily derived and $\nu$ equals to $1 / 2$.

In polymer melts, the repulsive interactions between monomers that expand the length of a polymer chain are the excluded volume interactions. The concept of "excluded volume" means that the volume of a monomer is not accessible 


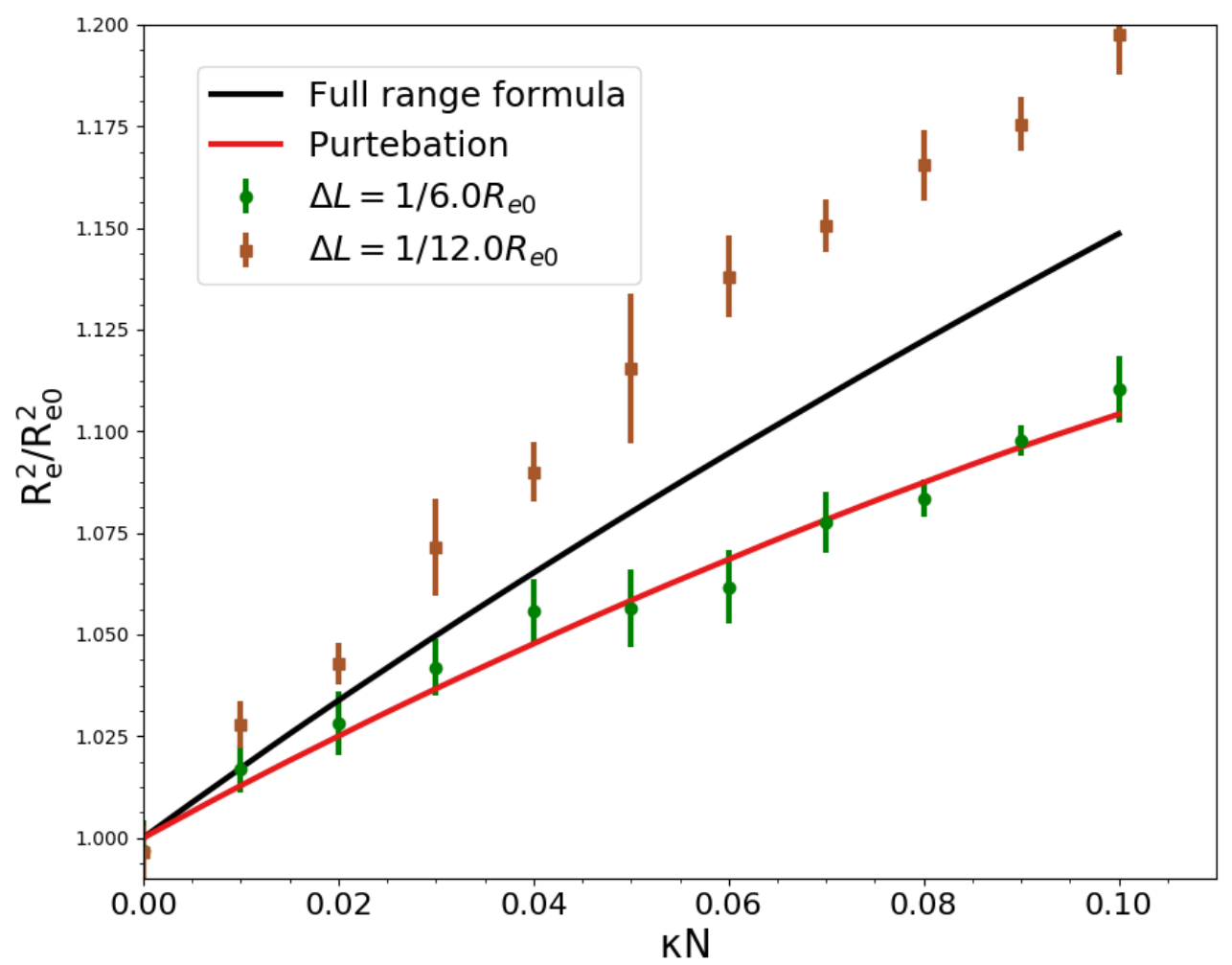

FIGURE 2.5: $R_{\mathrm{e}}^{2}$ is plotted as a function of $\kappa N$. We compare $R_{\mathrm{e}}^{2}$ as a function of excluded volume interaction parameter $\kappa N$ with the equations given by 2.42 and 2.44.

by other monomers. As a result, the mean-square end-to-end distance $R_{\mathrm{e}}^{2}$ will increase with the excluded volume interaction.

Numerical experimental studies show that the real chains in a solvent have the same universal features as the self-avoiding walk (SAW) model on a lattice. In mathematics, a SAW is a sequence of moves on a lattice (a lattice path) that does not visit the same point more than once. In three dimensions, the exponent $\nu$ is close to $3 / 5$ [47].

In the previous theoretical investigations [72-75], they investigate the singlechain expansion by a perturbation calculation using the Borel summation technique. In this subsection, we will numerically calculate the single-chain expansion by SCMF algorithm. 


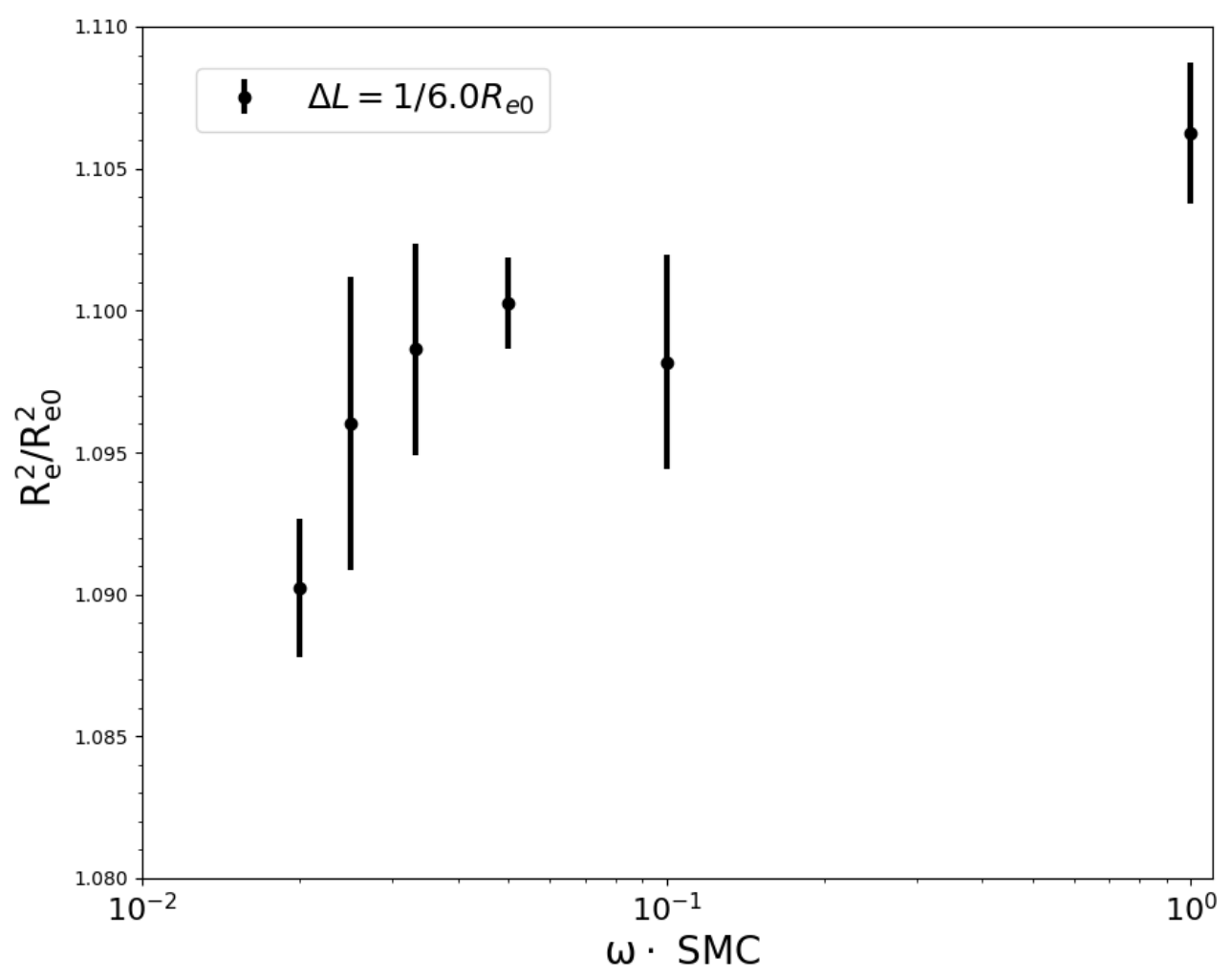

FIGURE 2.6: $R_{e}^{2}$ is plotted as a function of updating field frequency, $\omega$. The excluded volume interaction, $\kappa N=0.1$.

Suppose a single polymer in a solvent, the excluded volume interaction is given by $\mathcal{H}_{\text {exclude, }}$

$$
\frac{\mathcal{H}_{\text {excluded }}}{k_{\mathrm{B}} T}=\frac{1}{2} w \sum_{s=0}^{N} \sum_{s^{\prime}=0}^{N} \delta\left(\vec{r}(s)-\vec{r}\left(s^{\prime}\right)\right) .
$$

For the small excluded interaction parameter $w,\left\langle R_{e}^{2}\right\rangle$ can be written as a perturbation series [74],

$$
\begin{aligned}
\left\langle R_{\mathrm{e}}^{2}\right\rangle= & R_{\mathrm{e} 0}^{2}\left(1+\frac{4}{3} z-2.075 z^{2}+6.297 z^{3}-\right. \\
& \left.25.057 z^{4}+116.135 z^{5}-594.717 z^{6}+\ldots\right) .
\end{aligned}
$$

In this equation, the dimensionless interaction parameter $z$ is the Fixman parameter which is defined as,

$$
z \equiv\left(\frac{3}{2} \pi\right)^{3 / 2} w \frac{N^{2}}{R_{\mathrm{e} 0}{ }^{3}} .
$$


$z$ has a physical importance as the average number of collisions taking place in the polymer chain.

M. Muthukumar summarized the chain expansion equation throughout the full range of $z$ with the simple formula [75],

$$
\left\langle R_{\mathrm{e}}^{2}\right\rangle=R_{\mathrm{e} 0}{ }^{2} \exp \left\{0.1772 \ln \left(1+7.524 z+11.06 z^{2}\right)\right\} .
$$

Analytical formulas Eq.2.42 and Eq.2.42 slightly differs because of different approximation schemes [75].

To calculate the polymer chain expansion with our SCMF algorithm, we enforce the non-bonded interaction in Eq. 2.6 to be identical with the excluded volume interaction in Eq. 2.41. The incompatibility parameter, $\chi N$ is set to 0 and the incompressibility parameter, $\kappa N$ can be related to the strength of the excluded volume interaction $w$,

$$
w=\kappa N \frac{V}{N^{2}} .
$$

To relate the dimensionless parameter $z$ to the coarse-grained model, $z$ is represented as,

$$
z=\left(\frac{3}{2 \pi}\right)^{3 / 2} \kappa N \frac{V}{R_{\mathrm{e} 0}{ }^{3}} .
$$

In the simulation, we consider the simplest case that an isolated single polymer chain with 32 segments is confined to a volume $V=1 R_{\mathrm{e} 0} \times 2 R_{\mathrm{e} 0} \times 2 R_{\mathrm{e} 0}$ with a periodical boundary condition. We measure the mean-square end-to-end distance $R_{\mathrm{e}}^{2}$ every one thousand SMC. We update the instantaneous external fields $w_{\alpha}$ after each smart Monte Carlo step.

As shown in the Fig. 2.5, we plot the $R_{\mathrm{e}}^{2}$ as a function of the excluded volume interaction parameter $\kappa N$. We compare the results with theoretical predictions in Eq. 2.42 and Eq. 2.44. The results quantitatively agree with the theoretical prediction in [75].

The quality of the SCMF algorithm is controlled by the frequency of updating the external fields, $\omega$. This variable is defined as the number of updates per 


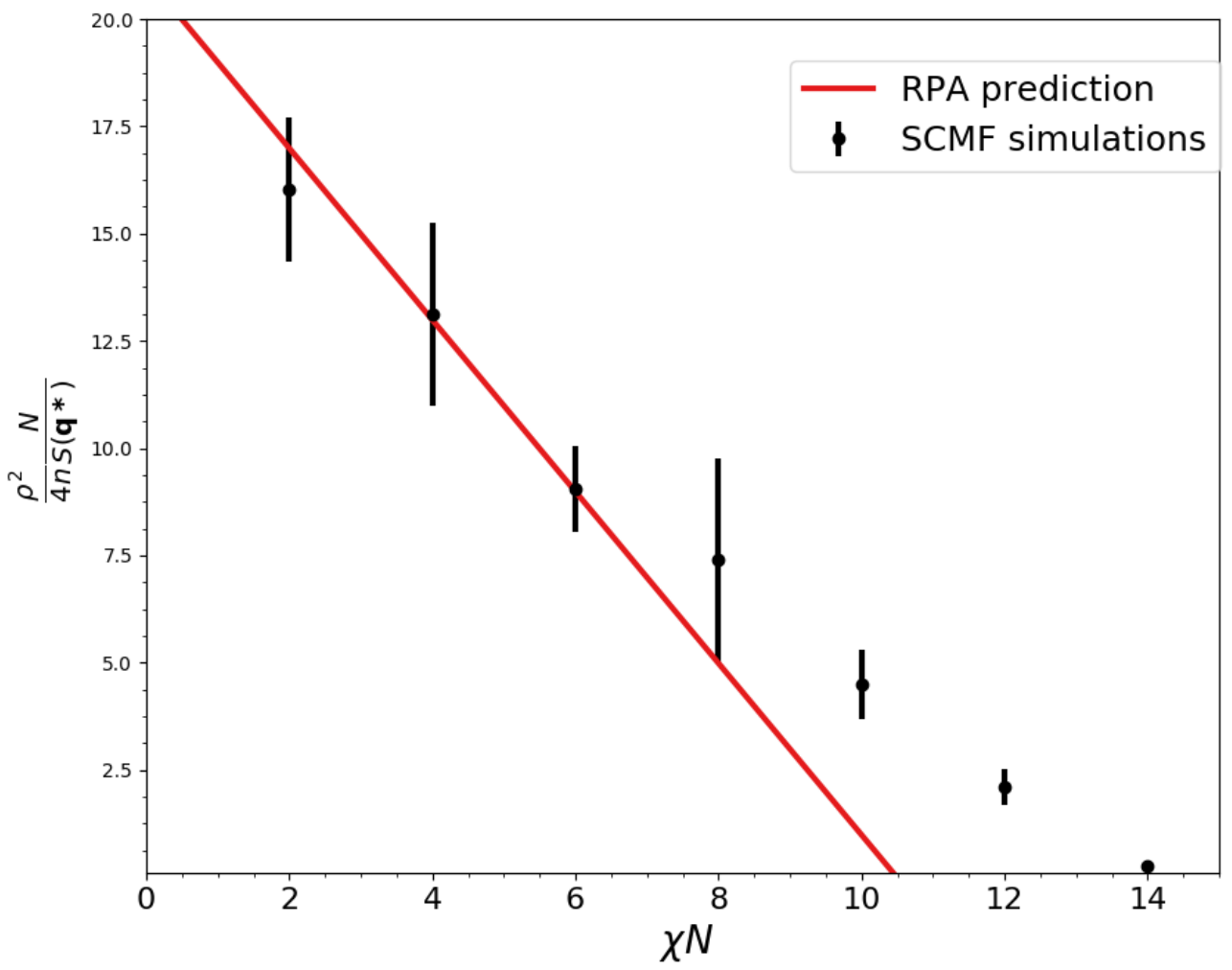

FIGURE 2.7: The reciprocal of the peak value of the structure factor of composition, $N / S\left(\left|q^{*}\right|\right)$ is plotted as a function of the incompatible parameter, $\chi N$.

SMC. We plot $R_{\mathrm{e}}^{2}$ as a function of the frequency $\omega$ in Fig. 2.6. The update frequencies which are above 1/10 updates per SMC are sufficient to capture the non-trivial correlation of an isolated chain.

By decreasing $\omega$, the chain expansion characterized by $R_{\mathrm{e}}^{2}$ decreases. In the limit of $\omega=0$, there is no update of the external fields. The mean-square, end-to-end distance, $R_{\mathrm{e}}^{2}$ gives an ideal chain result, i.e., $R_{\mathrm{e}}^{2}=R_{\mathrm{e} 0}^{2}$, which corresponds to SCFT approximation where the external fields are replaced by static external fields.

\subsubsection{Order-disorder transition of diblock copolymer melts}

In this subsection, we will use the SCMF algorithm to investigate the microphase separation of symmetric diblock copolymer melts. In the section 2.1, we use the Flory-Huggins theory investigate the phase separation of binary homopolymer 
blends. To describe a microphase separation for the symmetric diblock copolymer melts, one must take into account the long-range energies generated by the polymer chain connectivity.

The order-disorder transition (ODT) in diblock copolymers can be studied by the random-phase approximation (RPA). The transition point is predicted to be at $\chi N \approx 10.495$ within this mean-field approach by calculating the structure factor of the composition. Detailed calculations of the structure factor of the composition in the Appendix B [19]. Diblock copolymers belong to the Brazovskii universality class. The composition fluctuations alter the character of transition to first-order. Meanwhile, the position of the ODT point is also shifted. This phenomenon is observed by many simulations and experiments $[21,76]$.

Compared to the RPA approach, the advantage of the SCMF algorithm simulation is that the thermal fluctuations are also included. To investigate the composition fluctuations, we calculate the structure factor, $S(\mathbf{q})$ for various values of $\chi N$. The structure factor is defined as,

$$
S(\mathbf{q})=\int \mathrm{d} \vec{r}^{3} \mathrm{~d} \vec{r}^{\prime}\left\langle\hat{m}(\vec{r}) \hat{m}\left(\overrightarrow{r^{\prime}}\right)\right\rangle \exp \left\{-i \mathbf{q}\left(\vec{r}-\overrightarrow{r^{\prime}}\right)\right\} .
$$

$\langle\ldots\rangle$ represents the ensemble average. $\hat{m}(\vec{r})=\hat{\phi}_{A}(\vec{r})-\hat{\phi}_{B}(\vec{r})$ is the local composition in block copolymer melts. The structure factor of the composition $S(\mathbf{q})$ is the Fourier transform of the correlation function $\left\langle\hat{m}(\vec{r}) \hat{m}\left(\vec{r}^{\prime}\right)\right\rangle$.

This quantity can be measured by many experiment methods such as light, $\mathrm{X}$ ray, and neutron scattering. For symmetric diblock copolymers, $S(\mathbf{q})$ is zero at $|\mathbf{q}|=0$ and $\mathbf{q}=\infty$. $S(\mathbf{q})$ has a maximum for a certain value $\mathbf{q}^{*}$. When $\chi N$ do not exceed the ODT point, the peak value of $S\left(\mathbf{q}^{*}\right)$ is finite. As we increase the incompatibility parameter $\chi N$, the peak value $S\left(\mathbf{q}^{*}\right)$ increases. When $\chi N$ exceeds the ODT point, $S\left(\mathbf{q}^{*}\right)$ becomes infinite. The homogeneous phase becomes unstable and the microphase separation occurs spontaneously.

In the particle-based representation, the structure factor is,

$$
S(\mathbf{q})=\frac{1}{\rho^{2}}<\left|\sum_{i=1}^{n} \sum_{s=0}^{N}\left[\gamma_{A}(s)-\gamma_{B}(s)\right] e^{-i \mathbf{q} \vec{q}_{i}(s)}\right|^{2}>
$$


In our simulations, the volume of the simulation box is $V=1 \times 10 \times 10 R_{\mathrm{e} 0}{ }^{3}$. The discretization of the box $\Delta L=1 / 6 R_{\mathrm{e} 0}$. The number of the polymer in the box is 12800 . The invariant degree of polymerization $\overline{\mathcal{N}}$ equals to $128^{2}$.

The reciprocal peak values of the $S(\mathbf{q})$ is plotted as a function of Florry-Huggins parameter $\chi N$ in Fig. 2.7. The reciprocal peak height of the structure factor $N / S\left(\mathbf{q}^{*}\right)$ linearly depends on the $\chi N$ in small $\chi N$. At approximately $\chi N=14$, $N / S\left(\mathbf{q}^{*}\right)$ vanishes. From the RPA calculation, $N / S\left(\mathbf{q}^{*}\right)$ obeys

$$
\frac{\rho^{2}}{4 n} \frac{N}{S\left(\mathbf{q}^{*}\right)} \approx 2(10.945-\chi N) .
$$

We make a comparison of the SCFT simulation with the RPA calculation. The transition point is shifted to approximately $\chi N=14$. 


\subsection{Self-consistent field theory}

In the previous section 2.2, we introduce the soft, coarse-grained, particle-based model. By means of the SCMF algorithm, we can compute quantities, e.g., the mean-square end-to-end distance, $R_{\mathrm{e}}^{2}$, the average density of composition, $\langle\hat{m}\rangle=\left\langle\hat{\phi}_{A}-\hat{\phi}_{B}\right\rangle$, and the structure factor, $S(\mathbf{q})$. However, we cannot directly calculate the free energy of the system in a particle-based simulation. Because the free energy of the system is not an observable which is a function of particle coordinates.

In a fluid or self-assembling system, this difficulty is more significant which stems from the absence of a reference state for which the free energy can be accurately calculated. For instance, the crystal structure is a good reference state in the solid-state system because its free energy can be calculated rigorously. Non-interacting particles are attached to their ideal lattice positions by a harmonic spring potential. One can use the thermodynamic integration method to calculate the free-energy difference between the two thermal states [77]. However, in polymer systems, molecules are diffusing and cannot be constrained to the lattice position with a small excursion distance. One cannot easily find a good reference state.

For practical purposes, we need to compute the free energy of various morphologies and distinguish the thermodynamic equilibrium state from many metastable alternatives. In a particle-based simulation or an experiment, it may take protracted long time to reach the thermodynamic equilibrium. The free energy of the system is a good criterion to determine the thermodynamic equilibrium state.

In this section, we exploit that the free energy can be accurately calculated by SCFT in the limit of large $\overline{\mathcal{N}}$ and that our soft, particle-based model is very similar to the standard model of self-consistent field theory (SCFT). There are only two minor differences between a particle-based model and a continuum SCFT model: (i) In a standard SCFT model, the Gaussian chain contour is continuous. (ii) the local total density is constrained to unity, i.e., the system is strictly incompressible.

The Hamiltonian of the particle-based model in Sec. 2.2 is modified as, 


$$
\begin{aligned}
\frac{\hat{\mathcal{H}}_{\mathrm{scft}}}{k_{\mathrm{B}} T}= & \frac{3}{2 R_{\mathrm{e} 0}^{2}} \sum_{i=1}^{n} \int_{0}^{1} \mathrm{~d} s\left[\frac{\mathrm{d} \vec{r}_{i}(s)}{\mathrm{d} s}\right]^{2} \\
& -\ln \delta\left[1-\sum_{\alpha} \hat{\phi}_{\alpha}\right] \\
& -\frac{1}{2} \sqrt{\overline{\mathcal{N}}} \sum_{\alpha \neq \alpha^{\prime}} \int \frac{\mathrm{d}^{3} \vec{r}}{R_{\mathrm{e} 0}{ }^{3}} \chi_{\alpha, \alpha^{\prime}} N \hat{\phi}_{\alpha}(\vec{r}) \hat{\phi}_{\alpha^{\prime}}(\vec{r}) .
\end{aligned}
$$

The density of $\hat{\phi}_{A}(\vec{r})$ and $\hat{\phi}_{B}(\vec{r})$ is defined in Eq. 2.28.

In the following, we will introduce the saddle-point approximation to solve this model for the symmetric diblock copolymer melt.

\subsubsection{Method}

The partition function of the symmetric diblock copolymer melts in a canonical ensemble is,

$$
\mathcal{Z}_{\text {scft }}=\frac{1}{n !} \frac{1}{\lambda_{A}^{3 n N_{A}} \lambda_{B}^{3 n N_{B}}} \int \prod_{i=1}^{n} \prod_{s=0}^{1} \mathrm{~d} \vec{r}_{i}(s) e^{-\hat{\mathcal{H}}_{\mathrm{sct}} / k_{\mathrm{B}} T}
$$

where $i$ is the polymer index and $s$ is the segment index along the linear polymer. The polymer chain is discretized into $N_{s}+1$ segments and the index $s$ ranges from 0 to $1 . \lambda_{A}$ and $\lambda_{B}$ are the thermal de Broglie wavelength,

$$
\begin{aligned}
& \lambda_{A}=\frac{h_{\text {planck }}}{\sqrt{2 \pi m_{A} k_{\mathrm{B}} T}} \\
& \lambda_{B}=\frac{h_{\text {planck }}}{\sqrt{2 \pi m_{B} k_{\mathrm{B}} T}}
\end{aligned}
$$

where $m_{A}$ and $m_{B}$ is the mass of a single segment of the block copolymer.

The partition function $\mathcal{Z}_{\text {scft }}$ cannot be solved directly, because the interaction between molecules are not decoupled. To simply the calculation and make above partition function more tractable, people use a Hubbard-Stratonovich (HS) transformation to replace the many-body interaction terms by linear terms of auxiliary fields $W_{A}, W_{B}, \Phi_{A}, \Phi_{B}$ and $\Xi$ [17]. 
We insert the following equations in the partition function in Eq. 2.51,

$$
\begin{aligned}
\prod_{\vec{r}} \delta\left(1-\hat{\phi}_{A}(\vec{r})-\hat{\phi}_{B}(\vec{r})\right)= & \int \prod_{\vec{r}} \mathrm{~d} \Xi(\vec{r}) e^{\int \mathrm{d} \vec{r} \frac{\rho}{N} \Xi(\vec{r})\left(1-\hat{\phi}_{A}(\vec{r})-\hat{\phi}_{B}(\vec{r})\right)} \\
1= & \int \prod_{\vec{r}} \mathrm{~d} \Phi_{A}(\vec{r}) \delta\left[\hat{\phi}_{A}(\vec{r})-\Phi_{A}(\vec{r})\right] \\
1= & \int \prod_{\vec{r}} \mathrm{~d} \Phi_{B}(\vec{r}) \delta\left[\hat{\phi}_{B}(\vec{r})-\Phi_{B}(\vec{r})\right] \\
& \int \prod_{\vec{r}} \mathrm{~d} W_{B}(\vec{r}) \mathrm{d} \Phi_{B}(\vec{r}) \exp \left\{-\int \mathrm{d} \vec{r} \frac{\rho}{N} W_{B}(\vec{r})\left[\hat{\phi}_{B}(\vec{r})-\Phi_{B}(\vec{r})\right]\right\}
\end{aligned}
$$

The partition function, $\mathcal{Z}_{\text {scft }}$ in Eq. 2.51 is rewritten as,

$$
\begin{aligned}
\mathcal{Z}_{\text {scft }} \sim & \int \prod_{\vec{r}} d \Xi \prod_{\vec{r}} \mathrm{~d} W_{A} \prod_{\vec{r}} d W_{B} \prod_{\vec{r}} \mathrm{~d} \Phi_{A} \prod_{\vec{r}} \mathrm{~d} \Phi_{B} \\
& \int \prod_{i=1}^{n} \prod_{s=0}^{N_{s}} d \vec{r}_{i}(s) \exp \left[-\hat{H}_{b} / k_{\mathrm{B}} T-\int \mathrm{d} \vec{r} \frac{n}{V}\left(\hat{\phi}_{A} W_{A}+\hat{\phi}_{B} W_{B}\right)\right] \\
& \exp \left\{-\left[\int \mathrm{d} \vec{r} \chi \rho \Phi_{A} \Phi_{B}-\frac{\rho}{N} W_{A} \Phi_{A}-\frac{\rho}{N} W_{B} \Phi_{B}-\frac{\rho}{N} \Xi\left(1-\Phi_{A}-\Phi_{B}\right)\right]\right\} \\
= & \int \prod_{\vec{r}} \mathrm{~d} \Xi \prod_{\vec{r}} \mathrm{~d} W_{A} \prod_{\vec{r}} \mathrm{~d} W_{B} \prod_{\vec{r}} \mathrm{~d} \Phi_{A} \prod_{\vec{r}} \mathrm{~d} \Phi_{B} \mathcal{Q}^{n} \\
& \exp \left\{-\left[\frac{n}{V} \int \mathrm{d} \vec{r} \chi N \Phi_{A} \Phi_{B}-W_{B} \Phi_{B}-W_{A} \Phi_{A}-\Xi\left(1-\Phi_{A}-\Phi_{B}\right)\right]\right\} \\
= & \int \prod_{\vec{r}} \mathrm{~d} \Xi \prod_{\vec{r}} \mathrm{~d} W_{A} \prod_{\vec{r}} \mathrm{~d} W_{B} \prod_{\vec{r}} \mathrm{~d} \Phi_{A} \prod_{\vec{r}} \mathrm{~d} \Phi_{B} \exp \left\{-\frac{\mathcal{F}_{\mathrm{scft}}}{k_{\mathrm{B}} T}\right\} .
\end{aligned}
$$

$\mathcal{F}_{\text {scft }}$ is a functional of fields $\Phi_{A}, \Phi_{B}, W_{A}, W_{B}$ and $\Xi$ which is represented as,

$$
\frac{\mathcal{F}_{\text {scft }}}{n k_{\mathrm{B}} T}=-\ln \mathcal{Q}+\frac{1}{V} \int \mathrm{d} \vec{r}\left\{\chi N \Phi_{A}(\vec{r}) \Phi_{B}(\vec{r})-W_{B} \Phi_{B}-W_{A} \Phi_{A}-\Xi\left(1-\Phi_{A}-\Phi_{B}\right)\right\} .
$$


The functional $\mathcal{Q}\left[W_{A}, W_{B}\right]$ is the partition function of a single polymer chain under external fields $W_{A}$ and $W_{B}$,

$$
\begin{aligned}
\mathcal{Q}\left[W_{A}, W_{B}\right] \equiv & \int \prod_{s=0}^{1} \mathrm{~d} \vec{r}(s) \exp \left\{-\sum_{s=0}^{1} \frac{1}{\Delta s} \frac{3}{2 R_{\mathrm{e} 0}^{2}}[\vec{r}(s)-\vec{r}(s-1)]^{2}\right. \\
& \left.-\sum_{s=0}^{f_{A}} W_{A}[\vec{r}(s)] \Delta s-\sum_{s=f_{A}}^{1} W_{B}[\vec{r}(s)] \Delta s\right\} .
\end{aligned}
$$

$\Delta s=1 / N_{s}$ is the polymer chain discretization.

The interactions between polymers give rise to high-order terms in the partition function. In SCFT, these interactions are simplified by a saddle-point approximation. One employs the extreme point in the integrand as the equilibrium state and neglects the fluctuations.

The self-consistent equations are,

$$
\begin{aligned}
\frac{V}{n k_{\mathrm{B}} T} \frac{\delta \mathcal{F}_{\mathrm{sctt}}}{\delta \Xi} & =\Phi_{A}+\Phi_{B}-1=0 \\
\frac{V}{n k_{\mathrm{B}} T} \frac{\delta \mathcal{F}_{\mathrm{sctt}}}{\delta W_{A}} & =-\frac{V}{\mathcal{Q}} \frac{\delta \mathcal{Q}}{\delta W_{A}}-\Phi_{A}=0 \\
\frac{V}{n k_{\mathrm{B}} T} \frac{\delta \mathcal{F}_{\mathrm{sctt}}}{\delta W_{B}} & =-\frac{V}{\mathcal{Q}} \frac{\delta \mathcal{Q}}{\delta W_{B}}-\Phi_{B}=0 \\
\frac{V}{n k_{\mathrm{B}} T} \frac{\delta \mathcal{F}_{\mathrm{scft}}}{\delta \Phi_{A}} & =\Xi-W_{A}-\frac{\chi N}{2}\left(\Phi_{A}-\Phi_{B}\right)=0 \\
\frac{V}{n k_{\mathrm{B}} T} \frac{\delta \mathcal{F}_{\mathrm{scft}}}{\delta \Phi_{B}} & =\Xi-W_{B}-\frac{\chi N}{2}\left(\Phi_{B}-\Phi_{A}\right)=0 .
\end{aligned}
$$

The saddle-point approximation ignores thermal fluctuations around the mean value which are especially important at the weak-segregation regime.

To numerically solve nonlinear equation arrays from Eq. 2.60 to Eq. 2.64, the most essential procedure is to solve the single-chain partition function $\mathcal{Q}$.

We divide the partition function of the single chain $\mathcal{Q}$ by the partition function of an ideal chain $\mathcal{Q}_{0}$,

$$
\begin{aligned}
\mathcal{Q} / \mathcal{Q}_{0}= & \frac{1}{V} \int \mathrm{d} \vec{r}(1) e^{-W_{B}(\vec{r}(1)) \Delta s} \\
& \int \mathrm{d} \vec{r}(1) \Psi\left(\vec{r}(1), \vec{r}\left(1-1 / N_{s}\right)\right) e^{W_{B}(\vec{r}(1)) \Delta s} \\
& \cdots \quad \int \vec{r}(0) \mathrm{d} \Psi\left(\vec{r}\left(1 / N_{s}\right), \vec{r}(0)\right) e^{-\Delta s W_{A}(\vec{r}(0))} .
\end{aligned}
$$


Here $\Psi\left(\vec{r}, \overrightarrow{r^{\prime}}\right)$ is defined as the probability to transfer from the point $\vec{r}$ to the point $\overrightarrow{r^{\prime}}$.

$$
\Psi\left(\vec{r}, \overrightarrow{r^{\prime}}\right) \equiv e^{-\frac{a}{\Delta s}\left(\vec{r}-\vec{r}^{\prime}\right)^{2}} /\left(\frac{\pi \triangle s}{a}\right)^{\frac{3}{2}},
$$

where $a \equiv \frac{3}{2 R_{\mathrm{e} 0}{ }^{2}}$. From above definition, $\Psi\left(\vec{r}, \overrightarrow{r^{\prime}}\right)$ depends only on the distance between $\vec{r}$ and $\overrightarrow{r^{\prime}}$, so $\Psi\left(\vec{r}, \overrightarrow{r^{\prime}}\right)$ can also be written as $\Psi(\triangle r)$ and $\triangle r \equiv\left|\vec{r}-\overrightarrow{r^{\prime}}\right|$. The external field $W_{A}$ and $W_{B}$ is replaced by $W_{s}$,

$$
W_{s}= \begin{cases}W_{A} & \text { if } s<f_{A} \\ W_{B} & \text { if } s>f_{A}\end{cases}
$$

From the equation 2.65, the partition can be reformulated with a new functional $\mathbf{q}\left(\vec{r}, s ;\left[W_{s}\right]\right)$ of external field $W_{s}$,

$$
\begin{aligned}
\mathbf{q}\left(\vec{r}, s+\Delta s,\left[W_{s}\right]\right) & =e^{-W_{s}(r) \Delta s} \int \Phi\left(\vec{r}-\overrightarrow{r^{\prime}}\right) \mathbf{q}\left(\overrightarrow{r^{\prime}}, s,\left[W_{s}\right]\right) d \overrightarrow{r^{\prime}} \\
\mathbf{q}\left(\vec{r}, 0,\left[W_{s}\right]\right) & =e^{-W_{s}(\vec{r}) \Delta s}
\end{aligned}
$$

$\mathbf{q}\left(\vec{r}, s ;\left[W_{s}\right]\right)$ is the statistical weight for the bead $s$ of a chain at position $\vec{r}$. This quantity is also regarded as a chain propagator. The equation 2.68 is a Chapman-Kolmogorov equation. In the limit of a continuum chain limit $\Delta s \rightarrow 0$, this equation is reduced to the Fokker-Planck equation,

$$
\begin{aligned}
& \mathbf{q}\left(\vec{r}, s,\left[W_{s}\right]\right)+\frac{\partial}{\partial s} \mathbf{q}\left(\vec{r}, s,\left[W_{s}\right]\right) \Delta s \\
= & \left(1-W_{s}(\vec{r}) \Delta s\right) \int \Phi(\Delta r) \mathbf{q}\left(r+\Delta r, s,\left[W_{s}\right]\right) \mathrm{d} \Delta r \\
= & \left(1-W_{s}(\vec{r}) \Delta s\right) \int \Phi(\Delta r)\left(\mathbf{q}+\frac{\Delta r^{2}}{2} \nabla^{2} \mathbf{q}\right) \mathrm{d} \Delta r \\
= & \left(1-W_{s}(\vec{r}) \Delta s\right)\left(\mathbf{q}+\frac{\nabla^{2} \mathbf{q}}{2} \int_{0}^{\infty} e^{-\frac{a}{\Delta s} r^{2}} r^{3} d r\right) \\
= & \left(1-W_{s}(\vec{r}) \Delta s\right)\left(\mathbf{q}+\frac{1}{2} \frac{\Delta s}{2 a} \nabla^{2} \mathbf{q}\right) \\
\frac{\partial}{\partial s} \mathbf{q}= & \frac{\nabla^{2} \mathbf{q}}{4 a}-W_{s} \mathbf{q} .
\end{aligned}
$$

From above equations, the Fokker-Planck equation is, 


$$
\frac{\partial \mathbf{q}}{\partial s}= \begin{cases}\frac{R_{\mathrm{e} 0}{ }^{2}}{6} \nabla^{2} \mathbf{q}-W_{A} \mathbf{q} & \text { if } s<f_{A} \\ \frac{R_{\mathrm{e} 0}{ }^{2}}{6} \nabla^{2} \mathbf{q}-W_{B} \mathbf{q} & \text { else if } s>f_{A} .\end{cases}
$$

In the limit of continuum chain, the initial condition for $\mathbf{q}\left(\vec{r}, 0,\left[W_{s}\right]\right)$ reduces to

$$
\mathbf{q}\left(\vec{r}, 0,\left[W_{s}\right]\right)=1
$$

Because the two ends of the block copolymer are distinct. The opposite end segment distribution, $\mathbf{q}^{+}$is defined with a similar definition as $\mathbf{q}$. It satisfies $\mathbf{q}^{+}\left(\vec{r}, 1,\left[W_{s}\right]\right)=1$

The Fokker-Planck equation is also referred as a modified diffusion equation. The partition function $\mathcal{Q} / \mathcal{Q}_{0}$ can be represented by the statistical weight function $\mathbf{q}$ as,

$$
\frac{\mathcal{Q}}{\mathcal{Q}_{0}}=\frac{1}{V} \int \mathrm{d} \vec{r} \mathbf{q}\left(\vec{r}, 1 ;\left[W_{s}\right]\right)
$$

The density of the segment $\Phi$ can also be represent with $\mathbf{q}\left(\vec{r}, 1 ;\left[W_{s}\right]\right)$.

$$
\begin{aligned}
\Phi_{A} & =-\frac{\mathcal{Q}_{0}}{Q} \frac{\delta\left(\mathcal{Q} / \mathcal{Q}_{0}\right)}{\delta W_{A}} \\
& =\frac{1}{\mathcal{Q}} \sum_{0}^{f_{A}} \mathbf{q}\left(\vec{r}, s ;\left[W_{s}\right]\right) \mathbf{q}^{+}\left(\vec{r}, 1-s ;\left[W_{s}\right]\right) \\
\Phi_{B} & =-\frac{\mathcal{Q}_{0}}{\mathcal{Q}} \frac{\delta\left(\mathcal{Q} / \mathcal{Q}_{0}\right)}{\delta W_{A}} \\
& =\frac{1}{\mathcal{Q}} \sum_{f_{A}}^{1} \mathbf{q}\left(\vec{r}, s ;\left[W_{s}\right]\right) \mathbf{q}^{+}\left(\vec{r}, 1-s ;\left[W_{s}\right]\right)
\end{aligned}
$$

One thing needs to address is that in the limit of $\Delta s \rightarrow 0$, the double counting of the $\exp \{-W \Delta s\}$ is not important. In our discretized chain model, we use $N_{s}=100$ which gives $\Delta s=0.01$.

In the next section, we will illustrate the numerical implementation of the SCFT algorithm. 


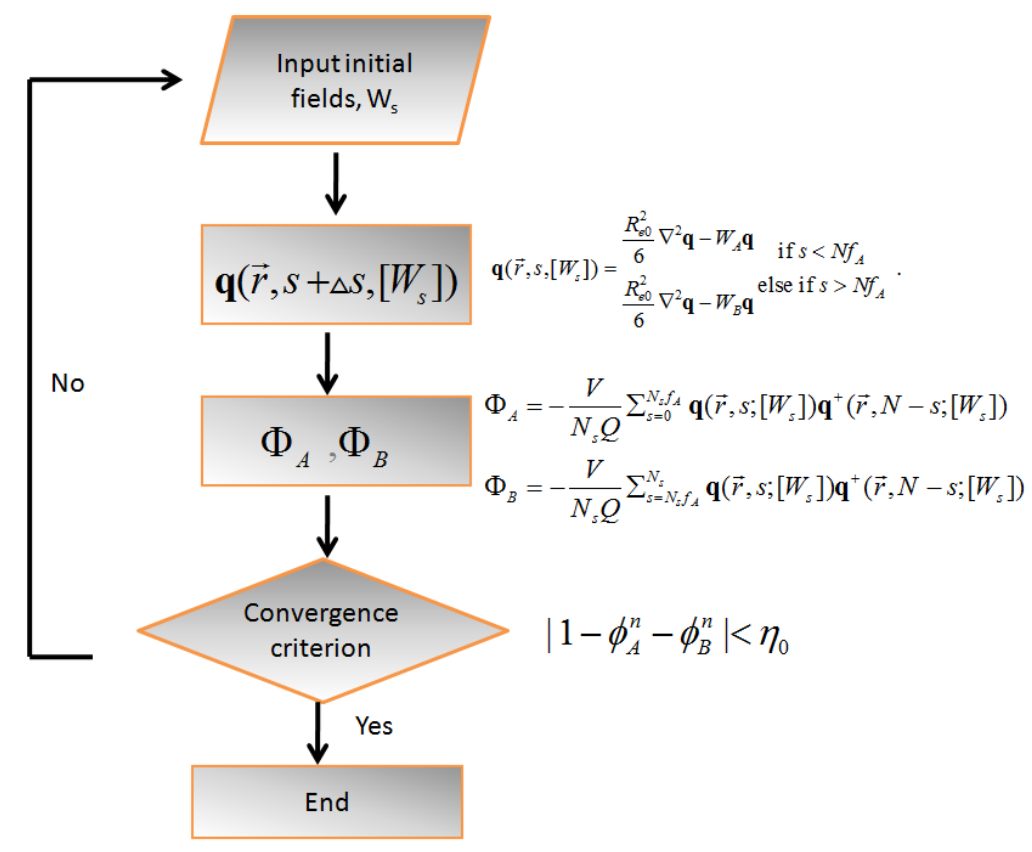

FIGURE 2.8: The flowchart of the SCFT algorithm.

\subsubsection{Numerical implementation}

In the numerical calculation of the SCFT iterative equations, the most essential and time-consuming step is to solve the diffusion equation 2.70. There are several numerical approaches to solve this differential equation. Generally, there are two main approaches, i.e., the reciprocal-space method and the real-space approach.

The reciprocal-space method or the spectral method takes advantage of the symmetry of the equilibrium structures [17]. One takes a set of complete and orthogonal functions as the basis function to numerically solve the modified diffusion equation. In analogy to solve the Schrödinger equation in the quantum mechanics, different modes are decoupled in the reciprocal space due to the spatial symmetry of the equilibrium state for block copolymers. The spectral method presumes the symmetry of the system, this method is not suitable to investigate meta-stable structure with defects. To exploit the meta-stable morphology in a nonequilibrium process, the real-space approach is comparably more suitable.

There are two main methods in the real-space approach, i.e., the finite-difference method and the pseudo-spectral method $[78,79]$. The finite-difference method 
can be implemented using an explicit scheme or an implicit scheme. The explicit scheme like the forward-time centered-space is the most straightforward scheme [80]. An implicit scheme like the Crank-Nicholson method is conditionally stable [81]. This scheme is more stable and accurate than the forward-time centered-space method. But the finite-difference method is difficult to be parallelly implemented [82].

To most practical method to numerically solve the diffusion equation 2.70 is the pseudo-spectral method [83]. This method is unconditional stable in all dimension and is more accurate than the finite-difference method. With parallel packages like FFTW and cuFFT, it is possible to implement the algorithm on the computer clusters and GPU with a high efficiency. In the following, we will employ this method to investigate various problems.

The exact expression $\mathbf{q}\left(\vec{r}, s+\Delta s,\left[W_{s}\right]\right)$ for the solution to the equation 2.70 is,

$$
\mathbf{q}\left(\vec{r}, s+\Delta s,\left[W_{s}\right]\right)=\exp \left[\left(R_{\mathrm{e} 0}^{2} \nabla^{2} / 6-W_{s}\right) \Delta s\right] \mathbf{q}\left(\vec{r}, s,\left[W_{s}\right]\right)
$$

The operator $\exp \left\{\left[R_{\mathrm{e} 0}{ }^{2} \nabla^{2} / 6-W_{s}\right] \Delta s\right\}$ can not be solved directly, because $\nabla^{2}$ and $W_{s}$ do not commute. This formula can be split with Baker-Campbell-Hausdorff formula. This splitting operation neglects high-order terms $o\left((\Delta s)^{2}\right)$,

$$
\begin{aligned}
& \exp \left\{-W_{s} \frac{\Delta s}{2}\right\}\left(\exp \left\{\Delta s R_{\mathrm{e} 0}^{2} \nabla^{2} / 6\right\} \exp \left\{-W_{s} \frac{\Delta s}{2}\right\}\right) \\
= & \exp \left\{-W_{s} \frac{\Delta s}{2}\right\} \exp \left\{\Delta s R_{\mathrm{e} 0}{ }^{2} \nabla^{2} / 6-W_{s} \frac{\Delta s}{2}+\frac{1}{2}\left[\Delta s \nabla^{2},-W_{s} \Delta s / 2\right]+o\left((\Delta s)^{2}\right)\right\} \\
= & \exp \left\{\Delta s R_{\mathrm{e} 0}{ }^{2} \nabla^{2} / 6-W_{s} \Delta s+o\left((\Delta s)^{2}\right\}\right. \\
\approx & \exp \left\{\Delta s R_{\mathrm{e} 0}{ }^{2} \nabla^{2} / 6-W_{s} \Delta s\right\}
\end{aligned}
$$

The pseudo-spectral method employs the Fourier transform FFT and inverse Fourier transform $F F^{-1}$ to solve above equation, 


$$
\begin{aligned}
& \exp \left\{\Delta s R_{\mathrm{e} 0}^{2} \nabla^{2} / 6-W_{s} \Delta s\right\} \\
\approx & \exp \left\{-W_{s} \frac{\Delta s}{2}\right\} F F T^{-1} F F T\left\{\exp \left(\Delta s R_{\mathrm{e} 0}^{2} \nabla^{2} / 6\right) \exp \left(-W_{s} \Delta s / 2\right)\right\} \\
= & \exp \left\{-W_{s} \frac{\Delta s}{2}\right\} F F T^{-1} \exp \left\{-\Delta s R_{\mathrm{e} 0}{ }^{2} k^{2} / 6\right\} F F T\left\{\exp \left(-W_{s} \Delta s / 2\right)\right\}
\end{aligned}
$$

The pseudo-spectral method is unconditionally stable and the accuracy is improved. This method allows for fewer spectral elements and a larger chain contour discretization.

We illustrate the algorithm of SCFT in the flowchart in Fig. 2.8. The SCFT equations are solved iteratively. The first step in the iterative procedures is to initialize the potential fields $W_{A}$ and $W_{B}$.

In the SCFT, we only consider the incompressible system. A given morphology is characterized by the local composition $m$ of $\mathrm{A}$ and $\mathrm{B}$ species. For a given morphology $m$, the potential fields are initialized as,

$$
\begin{aligned}
& W_{A}^{0}=(1-m) \chi N / 2 \\
& W_{B}^{0}=(1+m) \chi N / 2 .
\end{aligned}
$$

The second step is to substitute the fields $W_{A}^{n-1}$ and $W_{B}^{n-1}$ into the Eq. 2.70 and numerically solve the modified diffusion equations by the pseudo-spectral method given in Eq. 2.77. The Fourier transform can be implemented by the library of cuFFT on GPUs or the FFTW library on CPUs. After obtaining $\mathbf{q}\left(\vec{r}, s,\left[W_{s}\right]\right)$, the new density $\Phi_{A}^{n}$ and $\Phi_{B}^{n}$ are obtained by Eq. 2.73 .

The last step is to modify the field $W_{A}^{n}$ and $W_{A}^{n}$ with new density configuration $\phi_{A}^{n}$ and $\phi_{B}^{n}$,

$$
\begin{aligned}
& W_{A}^{n}=W_{A}^{n-1}+K_{\mathrm{co}}\left(\chi N \phi_{B}^{n}+\Xi-W_{A}^{n-1}\right) \\
& W_{B}^{n}=W_{B}^{n-1}+K_{\mathrm{co}}\left(\chi N \phi_{A}^{n}+\Xi-W_{B}^{n-1}\right) .
\end{aligned}
$$

The parameter $K_{\mathrm{co}}$ is a mixing parameter. We continue the iterative procedure until the SCFT equations are converged. One important convergence criteria is 
the incompressibility condition,

$$
\left|1-\phi_{A}^{n}-\phi_{B}^{n}\right|<\eta_{0}
$$

where $\eta_{0}$ is set as $10^{-8}$ in the program. .

\subsubsection{Performance}

In this section, we test the performance of the SCFT calculation with a parallel algorithm. To accelerate the SCFT calculation, we implement the algorithm on a distributed memory parallel cluster and the parallel computing GPUs with the MPI and CUDA techniques, respectively.

In 2013, Glenn H. Fredrickson and etc. make a similar implementation. They claim that the speedup of a double precision calculation on Tesla T20 is 30 compared to a single CPU core serial calculation [84].

As introduced in the above section, the modified diffusion equation is solved by employing the pseudo-spectral algorithm. The most time-consuming part of the calculation in this algorithm is to implement the Fourier transform. We use the parallel version of the FFTW library [85] and the cuFFT library [86] to implement the fast Fourier transform calculation.

The speed of the fast Fourier transform is determined by the size of the system, i.e., the number of grid points in the system. The chain discretization $\Delta s$ determines the number fast Fourier transforms during one iterative SCFT step. In this thesis, we set $\Delta s=1 / 100$, which needs 200 fast Fourier transforms during in one iterative step.

We first test the performance of the SCFT calculation on the GPU clusters. The time per iterative step (TPIS) with different system sizes are measured on different GPUs, e.g., the Tesla K80 and the Tesla P100, as shown in the table 2.3.

The computation time during one iterative step increases linearly with the system size, i.e., the total number of the grids $N_{g}$. Additionally, the efficiency of the SCFT algorithm on GPUs does not reach to the maximum with a small system 
TABLE 2.3: Performance of the SCFT algorithm on a single GPU.

\begin{tabular}{lll}
\hline Number of grid $N_{g}$ & TPIS on Tesla P100 $(\mathrm{ms})$ & TPIS on Tesla K80 $(\mathrm{ms})$ \\
\hline $16^{3}$ & 47.76 & 50.45 \\
$32^{3}$ & 51.47 & 60.40 \\
$64^{3}$ & 97.32 & 208.16 \\
$96^{3}$ & 320.44 & 799.92 \\
$128^{3}$ & 628.86 & 1529.88 \\
\hline
\end{tabular}

TABLE 2.4: Performance of the multiple SCFT calculations on a single GPU with a grid size $N_{g}=1 \times 480 \times 480$.

\begin{tabular}{lll}
\hline Number SCFT calculations & TPIS on Tesla P100 $(\mathrm{ms})$ & TPIS on Tesla K80 $(\mathrm{ms})$ \\
\hline 1 & 85.93 & 203.06 \\
2 & 138.92 & 381.91 \\
4 & 246.17 & 729.55 \\
8 & 453.63 & 1429.55 \\
16 & 1031.55 & 2835.60 \\
\hline
\end{tabular}

size. We can fully take advantage of the GPU capacity by parallelly implementing multiple SCFT calculations on one GPU.

We test the multiple SCFT calculations. The size of the system is $N_{g}=1 \times 480 \times$ 480. We present the results on the table 2.4. The reason for this implementation is that, we employ the string method to investigate collective phenomena which will be introduced in section 3.5.1. This algorithm implements multiple SCFT calculations during one iterative step.

In the end, we test the performance of MPI-based SCFT calculations on parallel clusters. The results are shown in the Fig. 2.5. The size of the system is $96 \times$ $96 \times 96$. The performance test is implemented on the Intel Xeon E5-2680 v3 Haswell CPU. The maximum speed-up factor is approximately 19.72. Because the parallel fast Fourier transform will transpose the matrix and frequently send and receive data. This process limits the efficiency of parallel algorithms.

As a comparison, it takes $320.44 m s$ on the Tesla P100 for one SCFT iterative step. The speed-up factor is approximately 35 . 
TABLE 2.5: Performance of the SCFT algorithm on multiple CPUs. The grid points of the system is $96 \times 96 \times 96$.

\begin{tabular}{lll}
\hline Number of cores & TPIS $(\mathrm{ms})$ & Speedup factor \\
\hline 1 & 11071.5 & 1 \\
2 & 6448.3 & 1.71 \\
4 & 3675.4 & 3.01 \\
8 & 2310.7 & 4.79 \\
16 & 1517.6 & 7.29 \\
24 & 993.7 & 11.14 \\
48 & 567.9 & 19.52 \\
96 & 561.3 & 19.72 \\
\hline
\end{tabular}

\subsection{A comparison of the particle-based SCMF algo- rithm simulation to the SCFT method}

In this section, we will compare the SCFT method to the SCMF algorithm in a symmetric binary AB homopolymer blend.

Equilibrium properties of block copolymer systems can be well described by the SCFT method. However, compared to the results from experiments or particlebased simulations, the physical properties obtained by the SCFT method are qualitatively correct but some important physical effects are missing. The aim of this section is to investigate thermodynamic properties in equilibrium, e.g., the phase transition in homopolymer blends, the interfacial width of phaseseparated homopolymer blends.

To make a comparison with the particle-based simulation, we can, on one hand, understand the difference between two numerical methods. On the other hand, we can examine the validity of our algorithms.

\subsubsection{AB binary homopolymer blends}

The demixing transition of the symmetric incompatible binary AB homopolymer blends is well studied by the mean-field theory [87]. We have derived and discussed the Florry-Huggins theory, which gives a transition point at $\chi N=2$ in the section 2.1. 


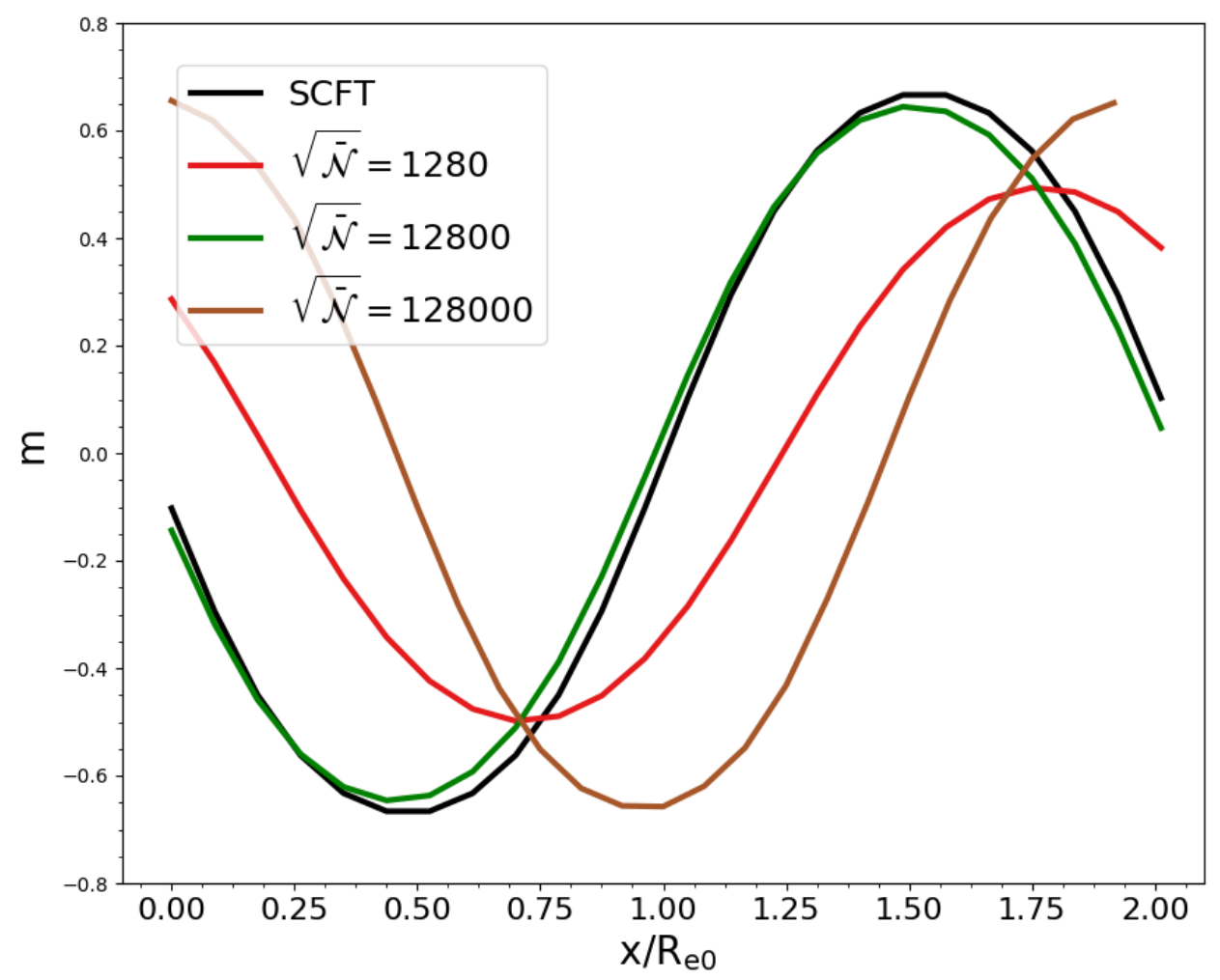

FIGURE 2.9: The density profile of $\mathrm{AB}$ polymer blends at $\chi N=4$ obtained by the SCFT and the SCMF, particle-based simulation. The SCFT results are compared to the particle-based simulation with $\sqrt{\mathcal{N}}=1600,16000$, and 160000 .

In this section, we will use the SCFT method and the particle-based, SCMF algorithm to investigate the equilibrium properties of symmetric binary homopolymer melts with the coarse-grained model (Sec. 2.2). The connectivity of the polymer backbone is described by a bead-spring model which is shown in Eq. 2.5. The non-bonded interactions for binary mixtures are identical to the diblock copolymers.

We implement the calculation in a quasi-one-dimensional system, $V=L \times R_{\mathrm{e} 0} \times$ $R_{\mathrm{e} 0}$. Above the critical point $\chi N=2$, the $\mathrm{A}$ and $\mathrm{B}$ polymers will separate into two phases along the $x$-axis. The SCFT calculation neglects the fluctuations, so we implement the SCFT calculation on a one-dimensional gird box with $L / \Delta L \times$ $1 \times 1$. In the SCMF algorithm, the polymerization of the polymer chain is $N=$ 32 and the space discretization is $\Delta L=1 / 12 R_{\mathrm{e} 0}$. In the SCFT, we use a finer polymer chain discretization, which gives $N_{s}=100$ and the space discretization is also $\Delta L=1 / 12 R_{\mathrm{e} 0}$. 
In Fig. 2.9, we compare the composition $m=\phi_{A}-\phi_{B}$ obtained from the SCFT method to the SCMF algorithm simulations along the $\mathrm{x}$ direction. The calculation is implemented at $\chi N=4$ with a box size $L=2 R_{\mathrm{e} 0}$. In the particle-based simulations, the density profile is homogeneous along the $\mathrm{y}$ and $\mathrm{z}$ direction, we average the concentration at $y$ - and $z$-directions. The small extension of the system will not significantly broaden the profiles by the capillary wave. In the simulation, the equilibrium state is obtained after running the simulation for $10^{5}$ SMCs.

From the snapshot, we find that the density profiles from the simulations deviate a little from the SCFT result. The small deviations origin from the thermal fluctuations, which is controlled by the invariant degree of polymerization, $\overline{\mathcal{N}}$.

The density profile from SCFT is more segregated because it corresponds to the limit of large $\overline{\mathcal{N}}$. The thermal fluctuations in particle-based simulations will shift the position of the critical point. As a result, the interface width $\omega_{\text {width }}$ which is defined by the inverse of the maximum slope of the density profile, also increases with $\overline{\mathcal{N}}$. When we use a very large $\overline{\mathcal{N}}$, the SCFT method and the particle-based simulations are quantitatively equivalent.

The symmetric binary homopolymer blends can also be well studied by the RPA calculation. The free-energy functional of symmetric $\mathrm{AB}$ homopolymer blends $\mathcal{F}_{\mathrm{RPA}}[m]$ is obtained by a Taylor expansion of the Flory-Huggins free-energy functional in Eq. 2.1 as,

$$
\frac{\mathcal{F}_{\text {blend }}^{\mathrm{RPA}}[m]}{k_{\mathrm{B}} T \sqrt{\overline{\mathcal{N}}}} \approx \int \frac{\mathrm{d}^{3} \vec{r}}{R_{\mathrm{e} 0}{ }^{3}}\left[\left(\frac{1}{2}-\frac{\chi N}{4}\right) m^{2}+\frac{1}{12} m^{4}+\frac{R_{\mathrm{e} 0}{ }^{2}}{36}(\nabla m)^{2}\right] .
$$

The free-energy functional $\mathcal{F}_{\text {blend }}^{\mathrm{RPA}}[m]$ comprises two parts: The first part is the bulk free energy of the coexisting phase. The latter part is the interfacial tension between the two interfaces.

To study the phase behavior with RPA method, we employ an Anzatz function, $A \sin (2 \pi x / L)$ as the equilibrium state, where $A$ is the the amplitude of the density. The RPA free-energy functional in Eq. 2.82 becomes a function $A$,

$$
\frac{\mathcal{F}_{\text {blend }}^{\mathrm{RPA}}(A)}{k_{\mathrm{B}} T \sqrt{\mathcal{N}}}=A^{2} L\left(\frac{2-\chi N}{8}\right)+\frac{3}{96} A^{4} L+\frac{A^{2} \pi^{2}}{18 L} .
$$




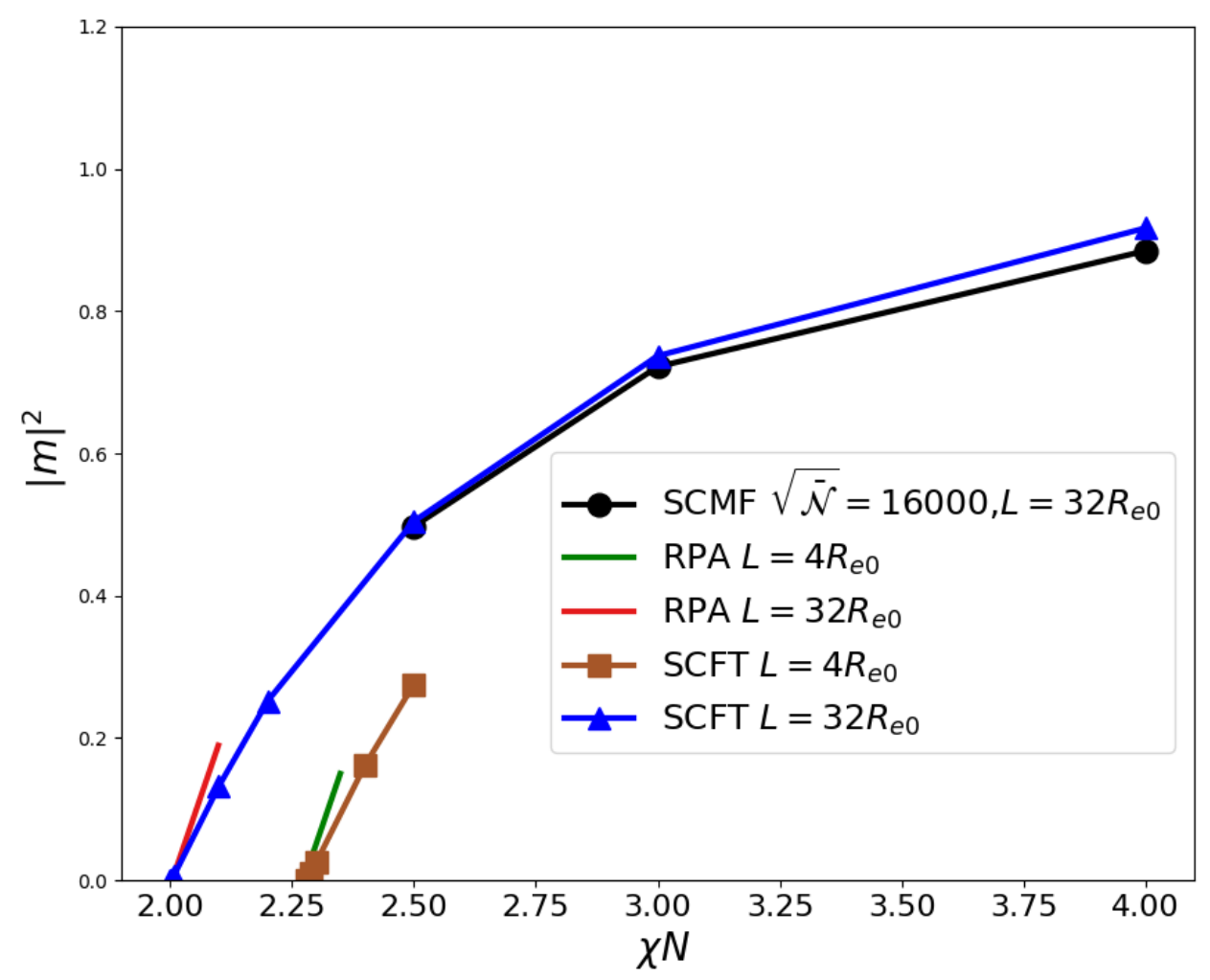

FIGURE 2.10: The maximum absolute value of $m$ is plotted as a function of the $\chi N$ along the $\mathrm{x}$ axis .

We plot the maximum absolute value of $m$ as a function of $\chi N$ in Fig. 2.10. In a mean-field theory, the critical point of the phase separation is at $\chi N=2$. Due to the finite-size effect, the critical point is shifted to $\chi^{*} N=2+4 \pi^{2} / 9 L^{2}$. In the vicinity of the transition, the order parameter $m$ gets smaller with an exponent $1 / 2$. The RPA calculation gives $m \propto \sqrt{\chi N-2-4 \pi^{2} / 9 L^{2}}$.

In the end, we investigate the width of the interface between A polymers and B polymers. It is defined as the maximum value of the inverse slope of the interface. In the strong-segregation limit (SSL), where $\chi N \gg 1$, The density profile is given by [88],

$$
m(x)=\tanh \left(x / \omega_{\mathrm{SSL}}\right) .
$$

The width of the interface is defined as,

$$
\omega_{\mathrm{SSL}}=\frac{R_{\mathrm{e} 0}}{\sqrt{6 \chi N}} .
$$


In equilibrium, the interfacial width decreases when the incompatible parameter, $\chi N$ increases. In Fig. 2.11, we plot the interfacial width as a function of $\chi N$. To minimize the finite-size effect, we implement the calculation with a large system size, $L=72 R_{\mathrm{e} 0}$. As expected, the interface becomes steeper when the $\chi N$ increases.

In the weak-segregation regime, we use the Ansatz function $\tanh \left(x / \omega_{\mathrm{RPA}}\right)$ to solve the free-energy functional in Eq. 2.82 to obtain the interfacial width, $\omega_{\mathrm{RPA}}$. By minimizing the free-energy at the interface, we obtain the interfacial width, $\tanh \left(x / \omega_{\mathrm{RPA}}\right)$ as,

$$
\omega_{\mathrm{RPA}}=\frac{R_{\mathrm{e} 0}}{3 \sqrt{\chi N-2}}
$$

We compare the results to the particle-based simulations and the SCFT in the Fig. 2.11. At smaller $\chi N$, both the particle-based simulations and the SCFT deviate a lot from the SSL prediction. When we increase $\chi N$, the deviation gets smaller. Due to thermal fluctuations, the interfacial width is larger in the particle-based simulations. 


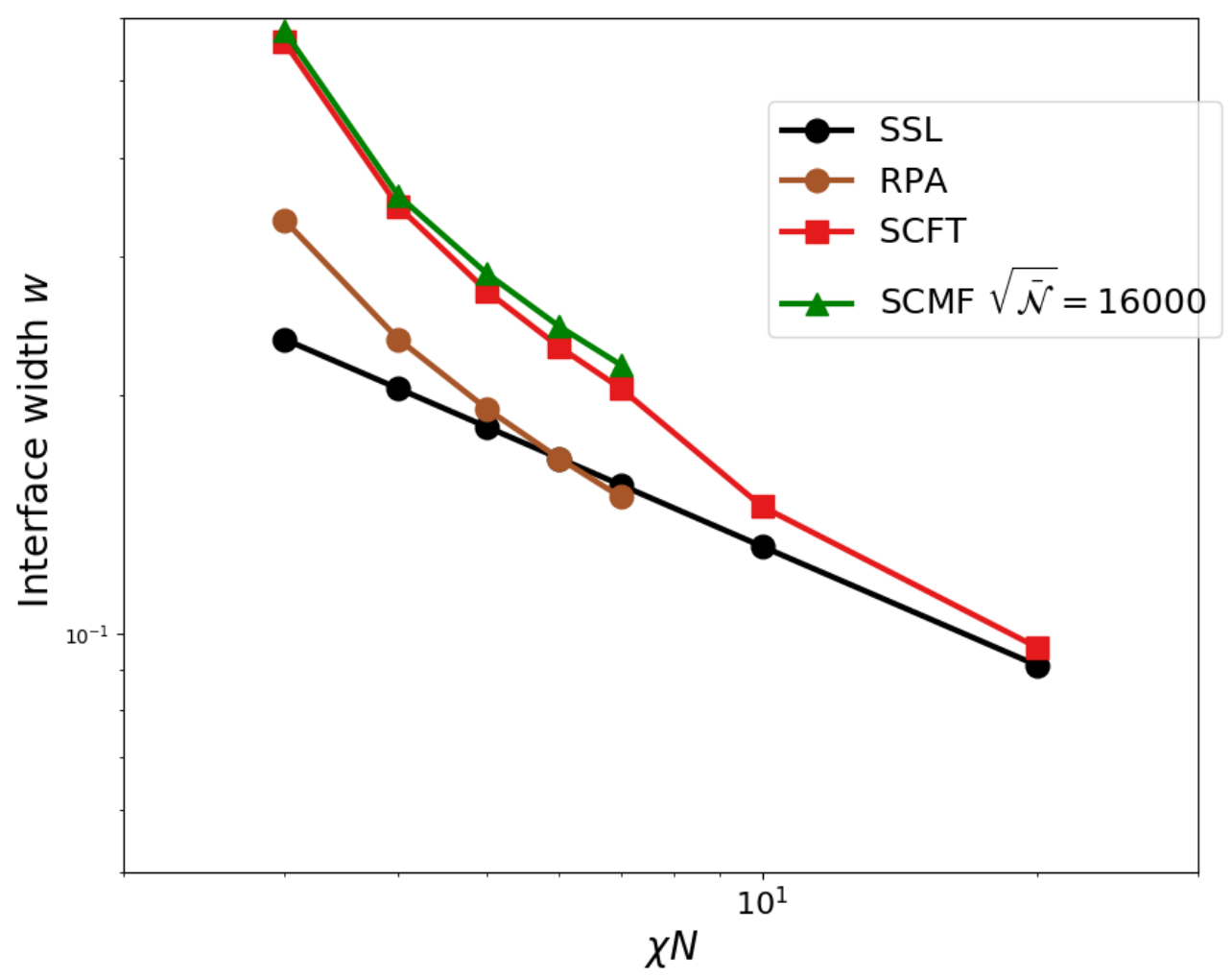

FIGURE 2.11: The interfacial width is plotted as a function of the incompatible parameter $\chi N$. The simulation results qualitatively agrees with the SCFT results when $\chi N$ increases. 


\section{Chapter 3}

\section{Free-energy landscape of block copolymers}

\subsection{Free-energy functionals}

Soft materials are characterized by free energies on the scale of the thermal energy, $k_{\mathrm{B}} T$. This energy scale is much smaller than the atomistic-scale, covalent bond energy scale, $\mathrm{eV} \approx 40 k_{\mathrm{B}} T$. Many collective phenomena (e.g., the self-assembly of block copolymers) which involves co-operative movements of many molecules are often dominated by a small free-energy difference between different thermal states.

In this sense, the most important information to predict the structure formation process in block copolymers is the free-energy landscape. The free-energy landscape is defined as a free-energy functional of the order parameter, $m$, which characterizes the configuration of the system.

The choice of the order parameter is crucial. In block copolymer melts, the molecules are not distinguishable. It is naturally inappropriate to use the coordinates of a specific portion of the molecules as the order parameter. A slow process in which the timescale to equilibrate the chain conformation is much smaller than the time scale of the structure formation process, usually employs the local densities, $\phi_{\alpha}$ as the order parameter. This order parameter is collective, i.e., it does not depend on a specific molecule which gives rise to the density at 
given point in space. Therefore it automatically accounts for the indistinguishable of identical molecules.

In a near-incompressible diblock copolymer melts or binary homopolymer blends, the order parameter $m$ is determined as,

$$
m \equiv \phi_{A}-\phi_{B}
$$

The definition of the free-energy functional $\mathcal{F}_{\text {target }}[m]$ for a specified $m$ is,

$$
\mathcal{F}_{\text {target }}[m] \equiv-k_{\mathrm{B}} T \ln \int \prod \mathrm{d} \vec{r}_{i}(s) \exp \left[-\frac{\hat{\mathcal{H}}_{b}+\hat{\mathcal{H}}_{n b}}{k_{\mathrm{B}} T}\right] \delta\left(m-\hat{\phi}_{A}+\hat{\phi}_{B}\right) \text {. }
$$

The calculation of free energies has many applications. For example, the phase diagrams of block copolymers (e.g., the phase diagram of diblock copolymer melts in Fig. 1.3) can be mapped out by comparing free energies of many metastable states. In a top-down, coarse-grained approach, the free energies are also used to determine invariant, coarse-grained parameters. For example, the Florry-Huggins parameter, $\chi N$ can be measured by matching the interfacial tension between domains.

Besides the free energies of stable and metastable states, the free energies of many transient, unstable states (i.e., the saddle points), which are found in the course of the structure formation process are also important. For example, we can describe many collective processes (e.g., the defect annihilation process) by a sequence of states, i.e., the minimum free-energy path (MFEP).

As discussed in the chapter 2, the calculation of the free energy for a block copolymer system is very challenging. In a particle-based simulation, one needs to find a reference state to determine the free-energy difference between the target state and the reference state. M. Müller and his colleges calculate the freeenergy difference of interfaces between lamellar morphologies with different orientations [89]. The free-energy difference is accurately obtained by a combination of the expanded-ensemble and the replica-exchange Monte Carlo technique. However, this method is rather computationally demanding. Alternatively, one can use field-theoretic umbrella sampling method, which is comparably efficient. This method restrains the fluctuations of a thermal state, $m$ by a harmonic umbrella potential. The thermodynamic driving force of the thermal 
state, $m$ can be accurately estimated [46]. This method has been successfully applied to study the bending rigidity of membrane [90]. Wang and Landau's algorithm is also studied to calculate free energy with the smart Monte Carlo method or the force-biased Monte Carlo method [91].

By means of the SCFT calculation, the free energies of metastable and stable states can be directly obtained by an iterative SCFT calculation. The free energies of unstable states can also be calculated by modifying the SCFT equations. Fredrickson and et al., propose a numerical scheme in SCFT to derive the thermodynamic forces for homopolymers [92], polymer blends and diblock copolymers that produce the lowest free energy spatial distribution of segments, subjected to the local incompressibility constraint. By going further, it is possible to construct numerical schemes that produce the lowest free energy spatial distribution, subjected to the local density constraint [93].

In this thesis, we will construct a numerical scheme in SCFT which mimics the field-theoretic umbrella sampling method in the particle-based simulation to derive the free energies and the thermodynamic forces in block copolymers. In fact, this numerical scheme is not limited to block copolymer materials, for example, this method is also used to compute the thermodynamic forces in the membranes [90].

In the following, we will first introduce numerical schemes to derive the chemical potential in SCFT and in particle-based simulations. To utilize the chemical potential, we will also investigate the mechanism of translating the chemical potential into the composition flow. The Onsager coefficient that connects the two parameters is measured in the particle-based simulations. In the end, we investigate the structure formation process in diblock copolymers and triblock copolymers by calculating the MFEP with the string method. 


\subsection{Numerical derivation of the chemical potential}

In this section, we will introduce several numerical schemes to derive the free energy and the chemical potential for a target state, $m$ in diblock copolymer melts or binary homopolymer blends.

We first introduce an analytical form of the chemical potential obtained by the RPA method. This analytical form of the chemical potential is only valid in the weak-segregation regime. A more accurate estimation can be made in the framework of the SCFT method. We construct iterative equations to "exactly" calculate the free-energy functional in Eq. 3.2. The disadvantage of this approach is that it is computationally demanding. To decrease the computational complexity, we use an umbrella potential to substitute the delta function constraint in equation 3.2. This scheme mimics the field-theoretic sampling method in the particle-based simulation.

In the end, we introduce the field-theoretic umbrella sampling method in the particle-based simulation and compare the results to the approaches in the framework of SCFT.

\subsubsection{Random-phase approximation}

The RPA free-energy functional for symmetric binary homopolymer blends is given in Eq. 2.82. We can obtain the corresponding chemical potential by a functional derivative which gives,

$$
\frac{\mu_{\mathrm{blend}}^{\mathrm{RPA}}[m] R_{\mathrm{e} 0}^{3}}{k_{\mathrm{B}} T \sqrt{\overline{\mathcal{N}}}}=\frac{R_{\mathrm{e} 0}^{3}}{k_{\mathrm{B}} T \sqrt{\overline{\mathcal{N}}}} \frac{\delta \mathcal{F}_{\mathrm{homo}}^{\mathrm{RPA}}[m]}{\delta m} \approx\left(1-\frac{\chi N}{2}\right) m-\frac{R_{\mathrm{e} 0}^{2}}{18} \Delta m .
$$

This RPA prediction is valid in the limit of a small amplitude variation $|m| \ll 1$ and a long wavelength, $q R_{\mathrm{e} 0} \ll 1$.

For $\mathrm{AB}$ diblock copolymer melts in the weak-segregation regime, the free-energy functional is [19],

$$
\frac{\mathcal{F}_{\text {diblock }}[m] R_{\mathrm{e} 0}^{3}}{k_{\mathrm{B}} T \sqrt{\overline{\mathcal{N}}}}=\frac{V}{2 R_{\mathrm{e} 0}^{3}} \sum_{q} \frac{|m(\mathbf{q})|^{2}}{g(1 / 2, x)-g(1, x) / 4},
$$


where $g\left(f_{A}, x\right)=\frac{2}{x^{2}}\left(e^{-f_{A} x}-1+f_{A} x\right)$ and $x=\left(q R_{\mathrm{e} 0}\right)^{2} / 6 . f_{A}$ is the composition of A blocks.

Taking the leading terms in the limit of $q R_{\mathrm{e} 0} \rightarrow 0$ and $q R_{\mathrm{e} 0} \rightarrow \infty$, the freeenergy functional takes the same expression as the Ohta-Kawasaki free-energy functional in Eq. 2.3.

More complex molecular architectures (e.g., branched polymers, multiblock, random or comb copolymers) give rise to more complex entropy functionals, which are difficult to derive with an approximation approach. It is of interest to determine those functionals in the framework of SCFT.

\subsubsection{Exact, delta-function constraint in SCFT}

In this section, we introduce an "exact" scheme to derive the chemical potential $\mu[m]$ for a target state $m$ in the framework of SCFT method. The chemical potential $\mu$ is defined as,

$$
\frac{R_{\mathrm{e} 0}^{3}}{\sqrt{\overline{\mathcal{N}}}} \frac{\mu[m]}{k_{\mathrm{B}} T} \equiv \frac{R_{\mathrm{e} 0}^{3}}{\sqrt{\overline{\mathcal{N}}}} \frac{\delta \mathcal{F}_{\text {target }}[m]}{k_{\mathrm{B}} T \delta m}
$$

We rewrite the free-energy functional 3.2 by the HS transformation with the equations 2.55. The additional delta-constraint function $\delta\left(m-\hat{\phi}_{A}+\hat{\phi}_{B}\right)$ is modified by introducing an additional field, $W_{\text {ex }}$ as,

$$
\delta\left(m-\hat{\phi}_{A}+\hat{\phi}_{B}\right)=\int \mathrm{d} W_{\text {ex }} \exp \left\{\int \mathrm{d} \vec{r} \frac{\rho}{N} W_{\text {ex }}\left(m-\hat{\phi}_{A}+\hat{\phi}_{B}\right)\right\} .
$$

The free-energy functional, $\mathcal{F}$ for a polymer chain is expressed as,

$$
\begin{aligned}
\frac{\mathcal{F}}{n k_{\mathrm{B}} T}= & -\ln \mathcal{Q}+\frac{1}{V} \int \mathrm{d} \vec{r}\left\{-\Xi\left(1-\Phi_{A}-\Phi_{B}\right)\right. \\
& +\chi N \Phi_{A} \Phi_{B}-W_{A} \Phi_{A}-W_{B} \Phi_{B} \\
& \left.+W_{\mathrm{ex}}\left(m-\Phi_{A}+\Phi_{B}\right)\right\} .
\end{aligned}
$$

$\mathcal{Q}$ is the single-chain partition function which is defined in equation 2.59 . 
Based on the saddle-point approximation, the self-consistent equations are obtained as,

$$
\begin{aligned}
\frac{V}{n k_{\mathrm{B}} T} \frac{\delta \mathcal{F}}{\delta \Xi} & =\Phi_{A}+\Phi_{B}-1=0 \\
\frac{V}{n k_{\mathrm{B}} T} \frac{\delta \mathcal{F}}{\delta W_{A}} & =-\frac{V}{\mathcal{Q}} \frac{\delta Q}{\delta W_{A}}-\Phi_{A}=0 \\
\frac{V}{n k_{\mathrm{B}} T} \frac{\delta \mathcal{F}}{\delta W_{B}} & =-\frac{V}{\mathcal{Q}} \frac{\delta \mathcal{Q}}{\delta w_{B}}-\Phi_{B}=0 \\
\frac{V}{n k_{\mathrm{B}} T} \frac{\delta \mathcal{F}}{\delta \Phi_{A}} & =\Xi-W_{A}+\chi N \Phi_{B}-W_{\mathrm{ex}}=0 \\
\frac{V}{n k_{\mathrm{B}} T} \frac{\delta \mathcal{F}}{\delta \Phi_{B}} & =\Xi-W_{B}+\chi N \Phi_{A}+W_{e x}=0 \\
\frac{V}{n k_{\mathrm{B}} T} \frac{\delta \mathcal{F}}{\delta W_{\mathrm{ex}}} & =m-\Phi_{A}+\Phi_{B}=0 .
\end{aligned}
$$

The free-energy $\mathcal{F}$ at saddle points, which is denoted as $\mathcal{F}_{\text {saddle }}$ is a functional of $m$. The functional $\mathcal{F}_{\text {target }}[m]$ is equivalent to the functional $\mathcal{F}$ at these saddle points,

$$
\mathcal{F}_{\text {target }}[m]=\mathcal{F}\left[\Xi, \Phi_{A}, \Phi_{B}, W_{A}, W_{B}, W_{\text {ex }}\right]=\mathcal{F}_{\text {saddle }}[m]
$$

The saddle-point values of $\Xi, \Phi_{A}, \Phi_{B}, W_{A}, W_{B}$, and $W_{\text {ex }}$ are functionals of $m$.

The chemical potential $\mu$ defined in equation 3.5 can be derived as,

$$
\frac{R_{\mathrm{e} 0}^{3}}{\sqrt{\mathcal{\mathcal { N }}}} \frac{\mu[m](\vec{r})}{k_{\mathrm{B}} T}=\frac{R_{\mathrm{e} 0}^{3}}{\sqrt{\mathcal{\mathcal { N }}}} \frac{\delta \mathcal{F}_{\text {target }}[m]}{k_{\mathrm{B}} T \delta m}=\frac{R_{\mathrm{e} 0}^{3}}{\sqrt{\overline{\mathcal{N}}}} \frac{\delta \mathcal{F}_{\text {saddle }}[m]}{k_{\mathrm{B}} T \delta m}=W_{\mathrm{ex}}[m] .
$$

We use an iterative approach to solve above functions. With the initial potentials $W_{A}^{0}$ and $W_{B}^{0}$, the $n^{\text {th }}$ densities $\Phi_{A}^{n}$ and $\Phi_{B}^{n}$ are obtained by,

$$
\begin{aligned}
\Phi_{A}^{n} & =-\frac{V}{\mathcal{Q}\left[W_{A}^{n-1}, W_{B}^{n-1}\right]} \frac{\delta \mathcal{Q}\left[W_{A}^{n-1}, W_{B}^{n-1}\right]}{\delta W_{A}^{n-1}} \\
\Phi_{B}^{n} & =-\frac{V}{\mathcal{Q}\left[W_{A}^{n-1}, W_{B}^{n-1}\right]} \frac{\delta \mathcal{Q}\left[W_{A}^{n-1}, W_{B}^{n-1}\right]}{\delta W_{B}^{n-1}}
\end{aligned}
$$

The parameters, $\Xi^{n}$ and $W_{\mathrm{ex}}^{n}$ are Lagrange multiplier that corresponds to the incompressibility and the order-parameter constraint which are obtained by,

$$
\begin{aligned}
\Xi^{n} & =\frac{1}{2}\left(W_{A}^{n-1}+W_{B}^{n-1}-\chi N\right) . \\
W_{\mathrm{ex}}^{n} & =\frac{1}{2}\left[-\chi N m-\left(W_{A}^{n-1}-W_{B}^{n-1}\right)\right] .
\end{aligned}
$$


After obtaining the fields $\Phi_{A}^{n}, \Phi_{B}^{n}, \Xi^{n}$, and $W_{\mathrm{ex}}^{n}$, the next step is to update the external fields, $W_{A}^{n}$ and $W_{B}^{n}$ with,

$$
\begin{aligned}
& \left.W_{A}^{n+1}=W_{A}^{n}+K_{\mathrm{co}}\left[\chi N(1-m) / 2+\Xi-W_{\mathrm{ex}}^{n}-W_{A}^{n}\right)\right] \\
& \left.W_{B}^{n+1}=W_{B}^{n}+k_{\mathrm{co}}\left[\chi N(1-m) / 2+\Xi+W_{\mathrm{ex}}^{n}-W_{B}^{n}\right)\right] .
\end{aligned}
$$

The parameter $K_{\mathrm{co}}$ is a mixing parameter, which is usually set to 0.01 in our calculation.

The iterative calculation ends when $\Phi_{A}^{n}$ and $\Phi_{B}^{n}$ satisfy,

$$
\begin{aligned}
& \eta_{\mathrm{com}}^{n}=\left|1-\Phi_{A}^{n}-\Phi_{B}^{n}\right|<10^{-8} \\
& \eta_{\mathrm{con}}^{n}=\left|m-\Phi_{A}^{n}+\Phi_{B}^{n}\right|<10^{-8} .
\end{aligned}
$$

$\eta_{\text {com }}^{n}$ and $\eta_{\text {con }}$ are incompressible constraint parameter and density constraint parameters, respectively.

With this technique, one can accurately calculate the chemical potential in the limit of $\overline{\mathcal{N}} \rightarrow \infty$. So this method is important to understand non-equilibrium phenomena and can be used to extend the field-theoretic model of SCFT into dynamics $[22,94,95]$.

\subsubsection{Umbrella potential method in SCFT}

In the previous section, we derive the chemical potential, $\mu[m]$ by using the saddle-point approximation on the free-energy functional in Eq. 3.2. This method can accurately map out the chemical potential within the mean-field approximation. However, a numerical implementation of this method is rather cumbersome. Because there are two Lagrange multiplier fields $W_{\text {ex }}$ and $\Xi$ in the self-consistent equations 3.8. It usually takes several magnitude more iterative steps than the numerical scheme with a single Lagrange multiplier field to converge.

To efficiently obtain the chemical potential with a controllable precision, we propose the umbrella potential method. This method resembles the field-theoretic umbrella sampling method in the Monte Carlo scheme [46]. We constrain the 
system to the target state $m$ by adding an umbrella potential $\mathcal{H}_{\lambda}$ which is defined as,

$$
\frac{1}{\sqrt{\mathcal{N}}} \frac{\mathcal{H}_{\lambda}}{k_{\mathrm{B}} T}=\frac{\lambda N}{2} \int \frac{\mathrm{d} \vec{r}}{R_{\mathrm{e} 0}^{3}}\left\{m-\hat{\phi}_{A}-\hat{\phi}_{B}\right\}^{2} .
$$

Similar to the previous treatment, we use the HS transformation to derive the free-energy functional $\mathcal{F}_{\lambda}$,

$$
\begin{aligned}
\frac{\mathcal{F}_{\lambda}\left[\Xi, \Phi_{A}, \Phi_{B}, W_{A}, W_{B}\right]}{n k_{\mathrm{B}} T}= & -\ln \mathcal{Q}+\frac{1}{V} \int \mathrm{d} \vec{r}\left\{\chi N \Phi_{A} \Phi_{B}-W_{A} \Phi_{A}-W_{B} \Phi_{B}\right. \\
& \left.-\Xi\left(1-\Phi_{A}-\Phi_{B}\right)-\frac{\lambda N}{2}\left(m-\Phi_{A}+\Phi_{B}\right)^{2}\right\} .
\end{aligned}
$$

The iterative equations are obtained as,

$$
\begin{aligned}
\frac{V}{n k_{\mathrm{B}} T} \frac{\delta \mathcal{F}_{\lambda}}{\delta \Xi} & =\Phi_{A}+\Phi_{B}-1=0 \\
\frac{V}{n k_{\mathrm{B}} T} \frac{\delta \mathcal{F}_{\lambda}}{\delta W_{A}} & =-\frac{V}{\mathcal{Q}} \frac{\delta \mathcal{Q}}{\delta W_{A}}-\Phi_{A}=0 \\
\frac{V}{n k_{\mathrm{B}} T} \frac{\delta \mathcal{F}_{\lambda}}{\delta W_{B}} & =-\frac{V}{\mathcal{Q}} \frac{\delta \mathcal{Q}}{\delta W_{B}}-\Phi_{B}=0 \\
\frac{V}{n k_{\mathrm{B}} T} \frac{\delta \mathcal{F}_{\lambda}}{\delta \Phi_{A}} & =\Xi-W_{A}+\chi N \Phi_{B}-\lambda N\left(m-\Phi_{A}+\Phi_{B}\right)=0 \\
\frac{V}{n k_{\mathrm{B}} T} \frac{\delta \mathcal{F}_{\lambda}}{\delta \Phi_{B}} & =\Xi-W_{B}+\chi N \Phi_{A}+\lambda N\left(m-\Phi_{A}+\Phi_{B}\right)=0 .
\end{aligned}
$$

The free-energy functional $\mathcal{F}_{\lambda}$ at its saddle points $\mathcal{F}_{\text {saddle }}$ can be regarded as a functional of $m$. In the limit of $\lambda N \rightarrow \infty$, the umbrella potential constraint is equivalent to the delta-function constraint. As a result, the free-energy functional $\mathcal{F}_{\text {saddle }}[m]$ at its saddle points can be approximately used as the $\mathcal{F}_{\text {target }}[m]$, i.e.,

$$
\lim _{\lambda N \rightarrow \infty} \mathcal{F}_{\text {saddle }}[m]=\mathcal{F}_{\text {target }}[m] .
$$

The strength of the umbrella potential is controlled by the parameter $\lambda N$. This parameter must be set to a value which is large enough to constrain the order parameter, $m$. In the next section, we will discuss the influence of the parameter $\lambda N$ to the numerical precision. 
Similarly, the chemical potential $\mu_{\lambda}$ of the free-energy functional $\mathcal{F}_{\lambda}[m]$ is,

$$
\frac{R_{\mathrm{e} 0}{ }^{3}}{\sqrt{\overline{\mathcal{N}}}} \frac{\mu_{\lambda}}{k_{\mathrm{B}} T}=\frac{R_{\mathrm{e} 0}{ }^{3}}{\sqrt{\mathcal{\mathcal { N }}}} \frac{\delta \mathcal{F}_{\text {saddle }}[m]}{k_{\mathrm{B}} T \delta m}=\lambda N\left(m-\Phi_{A}+\Phi_{B}\right)
$$

In the limit of $\lambda N \gg 1$, we use the approximation $\mu_{\lambda} \approx \mu$.

The system is incompressible, the choice of the umbrella potential is not unique. In the appendix A, we briefly introduce several equivalent umbrella potentials and derive their corresponding iterative equations.

\subsubsection{Field-theoretic umbrella sampling in the particle-based simulation}

In this section, we will introduce the umbrella sampling method in conjunction with the SCMF algorithm in the particle-based simulation. The soft, coarsegrained, particle-based model in the section 2.2 is equivalent to the SCFT model introduced in section 2.4 when the total density fluctuations and the composition fluctuations are completely suppressed, i.e., the parameter $\kappa N$ and $\overline{\mathcal{N}}$ are infinitely large.

Unlike in the SCFT calculation, the phase space of the target state, $m$ cannot be sampled by using a harsh delta-function constraint in a particle-based simulation. Because a trial move of the particle will give rise to a small variation of the local, microscopic density profile and violate the harsh, delta-function constraint. As a result, such a harsh, delta-function constraint will give rise to sampling problems in the particle-based simulations and results in a low acceptance rate.

To solve this difficulty, the harsh, delta-function constraint is usually substituted by an umbrella potential. In this section, we use an umbrella potential $\mathcal{H}_{\lambda}$ which is given in Eq. 3.24.

The chemical potential $\mu$ of the thermal state, $m$ in a particle-based simulation is derived as,

$$
\frac{R_{\mathrm{e} 0}^{3}}{\sqrt{\overline{\mathcal{N}}}} \frac{\mu[m]}{k_{\mathrm{B}} T} \approx \frac{R_{\mathrm{e} 0}^{3}}{\sqrt{\overline{\mathcal{N}}}} \frac{\mu_{\lambda}[m]}{k_{\mathrm{B}} T}=\lambda N\left(m-\left\langle\hat{\phi}_{A}-\hat{\phi}_{B}\right\rangle_{\lambda N}\right)
$$


The thermodynamic average $\langle\ldots\rangle_{\lambda N}$ is defined as,

$$
\langle\ldots\rangle_{\lambda N}=\int \mathcal{D}\left\{\vec{r}_{i}(s)\right\} \ldots \exp \left[-\frac{\mathcal{H}_{b}+\mathcal{H}_{n b}+\mathcal{H}_{\lambda N}}{k_{\mathrm{B}} T}\right]
$$

In the limit of a large $\lambda N$, this approximation becomes exact. However, we cannot use a very large value of $\lambda N$ in a particle-base simulation. Because the umbrella potential will give rise to a large force on the molecular dynamics. In the following section, we will discuss how to determine a proper $\lambda N$ in the simulation.

In the end, we can estimate the free-energy difference, $\Delta \mathcal{F}$ between two states by measuring the chemical potential. We consider a sequence of states $m_{\alpha}$, which is indexed by $\alpha$. The free-energy difference, $\Delta \mathcal{F}$ between two states, $m_{\alpha=0}$ and $m_{\alpha=1}$ can be estimated as,

$$
\frac{\Delta \mathcal{F}}{k_{\mathrm{B}} T}=\int_{\alpha=0}^{\alpha=1} \mathrm{~d} \alpha \int \mathrm{d} \vec{r} \frac{\delta \mathcal{F}\left[m_{\alpha}\right]}{\delta m_{\alpha}} \frac{\partial m_{\alpha}}{\partial \alpha}
$$

In a discretization form, $\Delta \mathcal{F}$ is approximately calculated as,

$$
\frac{R_{\mathrm{e} 0}^{3}}{\sqrt{\overline{\mathcal{N}}}} \frac{\Delta \mathcal{F}}{k_{\mathrm{B}} T} \approx \int \mathrm{d} \vec{r} \sum_{\alpha} \frac{\left(\mu\left[m_{\alpha+\Delta \alpha}\right]+\mu\left[m_{\alpha}\right]\right)}{2}\left(m_{\alpha+\Delta \alpha}-m_{\alpha}\right) \Delta \alpha .
$$

\subsubsection{Numerical results}

In this section, we will make a comparison of various numerical methods, which are introduced in the previous sections by computing the chemical potential.

\subsubsection{A comparison between the RPA method and the exact results ob- tained by the delta-function constraint in conjunction with SCFT}

To utilize the RPA approximation, we try to obtain the chemical potential of a one-dimensional density profile, $m=A \sin (q x)$ for a symmetric binary homopolymer blend. The incompatible parameter, $\chi N$ is set to 0 which corresponds to a homogeneous state in equilibrium. 


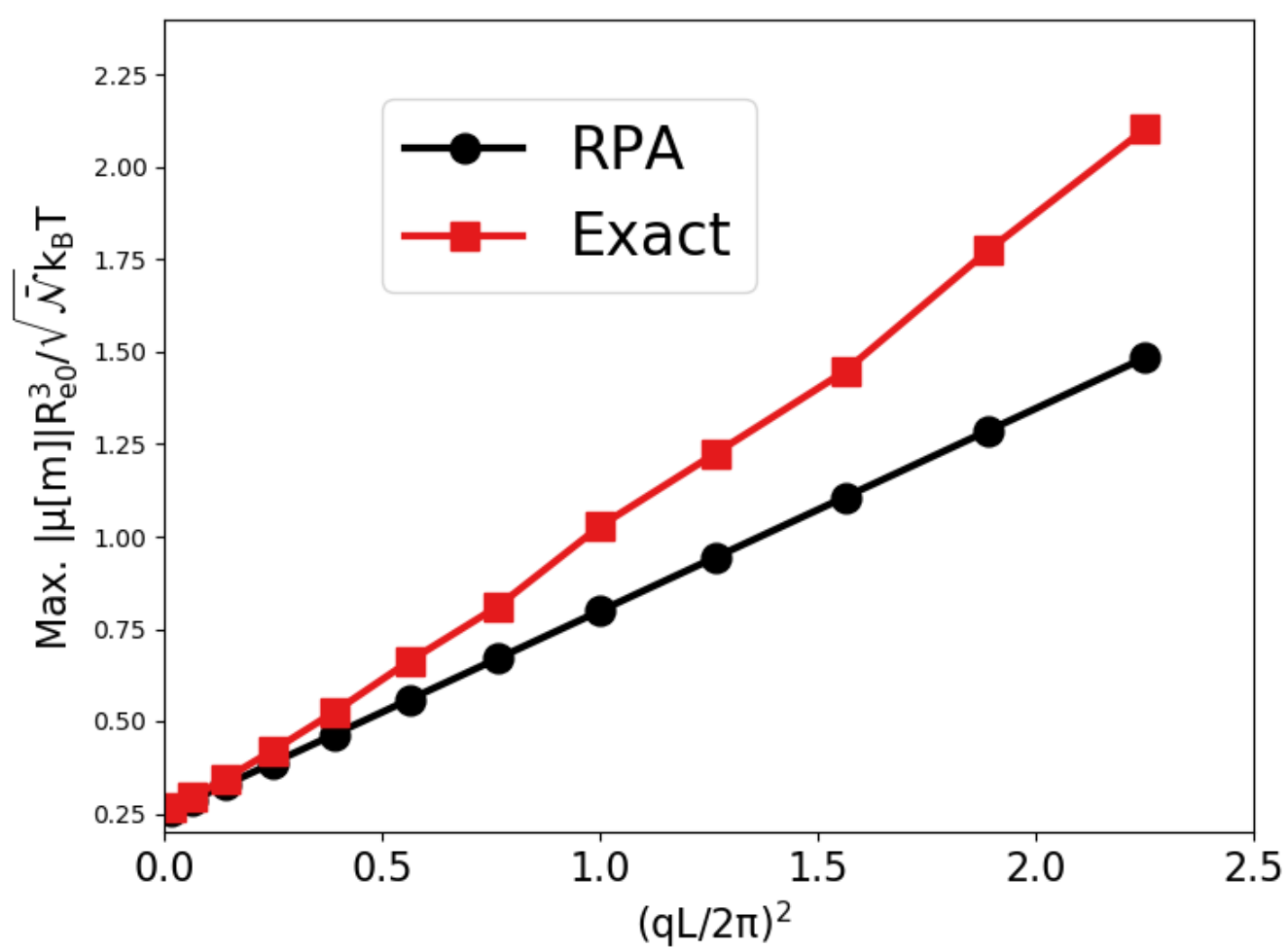

FIGURE 3.1: A comparison between the exact chemical potential and the RPA results. The exact results are obtained by the delta-function constraint method in SCFT.

The amplitude of the target density profile, $A$ is a small value which equals to 0.25 . The extension of the system is $L=8 R_{\mathrm{e} 0}$ and $n_{q}$ ranges from 1 to 12 . By the Eq. 3.3, the chemical potential is obtained as,

$$
\frac{\mu_{\mathrm{blend}}^{\mathrm{RPA}}[m](x) R_{\mathrm{e} 0}^{3}}{k_{\mathrm{B}} T \sqrt{\overline{\mathcal{N}}}}=A \sin (q x)+A R_{\mathrm{e} 0}^{2} q^{2} \sin (q x) / 18 .
$$

The chemical potential, $\mu_{\text {blend }}^{\mathrm{RPA}}[m](x)$ is a sine function, which has an identical Fourier mode, $q R_{\mathrm{e} 0}$ to the density, $m$. So we only need to compare the amplitude of the chemical potential of various numerical methods.

As shown in Fig. 3.1, we measure the maximal absolute value of the chemical potential as a function of the wave factor, $q R_{\mathrm{e} 0}$. The chemical potential, $\mu_{\text {blend }}^{\mathrm{RPA}}[m](x)$ obtained from the RPA approach agrees well with the SCFT predictions at a small $q R_{\mathrm{e} 0}$. At a large $q R_{\mathrm{e} 0}$, the RPA underestimates the strength of the chemical potential. In this case, the deviation at $q R_{\mathrm{e} 0} / 2 \pi=1.5$ is approximately $41 \%$. 


\subsubsection{An analysis of the umbrella potential method in conjunction with SCFT}

The RPA chemical potential provides an analytical form of chemical potential in the limit of a small variation of the order parameter, i.e., $|m| \ll 1$ and the long wavelength, $q R_{\mathrm{e} 0} \ll 1$. In a strongly segregated system, this method is not appropriate. In this section, we try to calculate the thermodynamic forces at the interface by using the umbrella potential method in conjunction with SCFT.

Consider a symmetric diblock copolymer melt in a one-dimensional volume, $V=L \times R_{\mathrm{e} 0} \times R_{\mathrm{e} 0}$, the extension of the system along the $\mathrm{x}$-axis is $L=1.5 R_{\mathrm{e} 0}$, which is approximately commensurate with the lamellar spacing of symmetric diblock copolymers at $\chi N=20$. In the SCFT, the fluctuations of the density are neglected, so we do not need to take into account the fluctuations on the $y$ and z-axis. The number of the grid points at y and z-axis is set as $N_{y}=1$ and $N_{z}=1$, respectively. The chain discretization in SCFT method is $N_{s}=100$.

In this section, we will generate a target morphology with a sharp interface by the umbrella potential method in SCFT and calculate its the thermodynamic forces at the interface. The target morphology with a sharp interface is generated via a step function, $m_{\text {step }}$ defined as,

$$
m_{\text {step }}(x)= \begin{cases}1 & x>0.75 R_{\mathrm{e} 0} \\ -1 & x<0.75 R_{\mathrm{e} 0}\end{cases}
$$

The step function cannot be directly used as the target state to study the thermodynamic forces at the interface of the diblock copolymer melts. We generate the target density profile $m_{\text {target }}$ by constraining the system to $m_{\text {step }}$ with a soft umbrella potential in the SCFT. The strength of the umbrella potential is set as $\lambda N=50$. The resulting density $m_{\text {target }}$ has a "softer" interface and is used as the target density to calculate the free energy and the thermodynamic force. The density profile of $m_{\text {target }}$ and $m_{\text {step }}$ is shown in Fig. 3.2.

In Fig. 3.3, we plot the chemical potential, $\mu_{\lambda N}\left[m_{\text {target }}\right](x)$ with different values of $\lambda N$. Compared to the exact result, $\mu_{\text {exact }}\left[m_{\text {target }}\right](x)$, the chemical potential, $\mu_{\lambda N}\left[m_{\text {target }}\right](x)$ obtained by the umbrella potential method underestimates the strength of the chemical potential, especially at the interface. The reason is that 


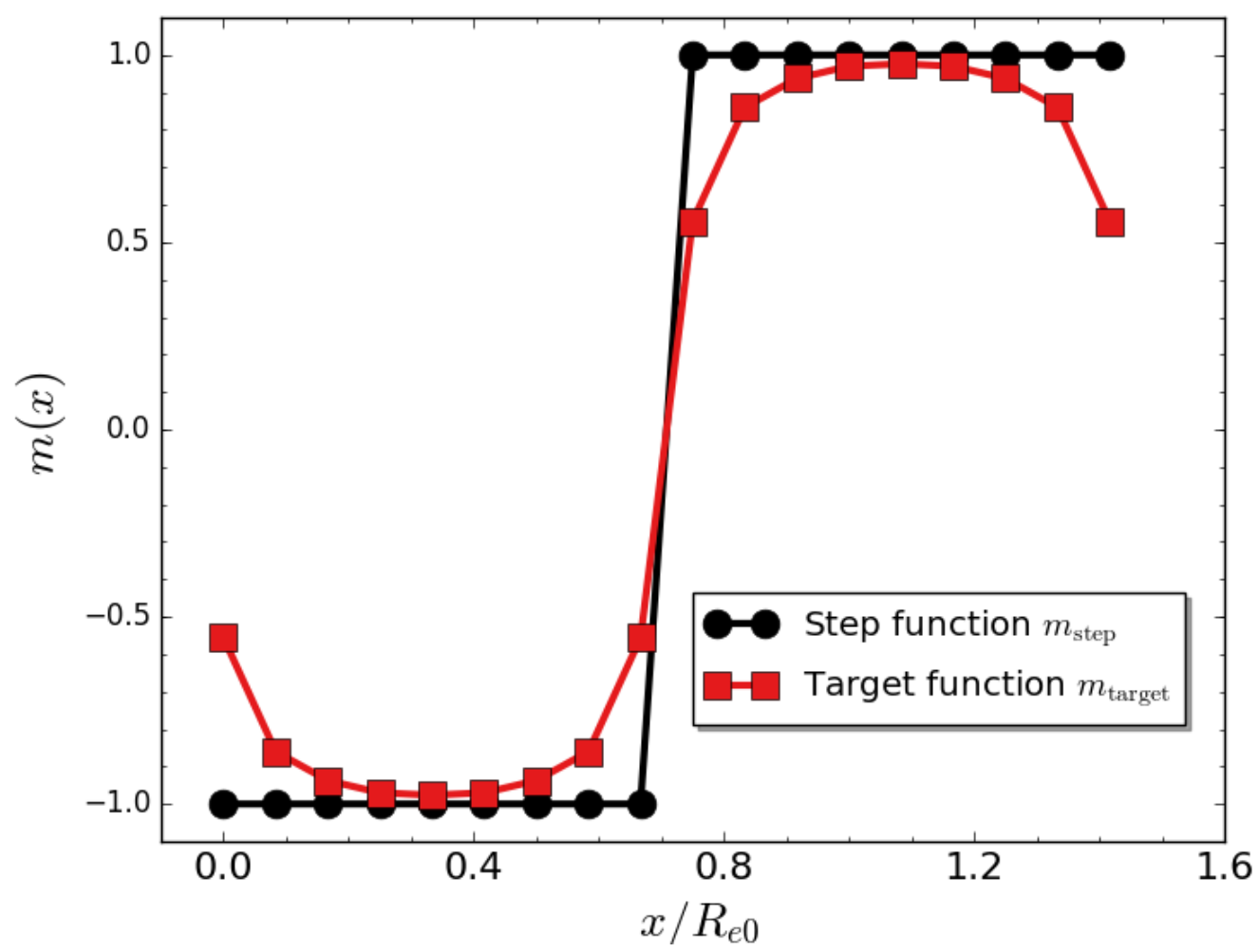

FIGURE 3.2: The density profile of diblock copolmers $m_{\text {step }}$ and $m_{\text {target }}$ along the $\mathrm{x}$ axis. $m_{\text {target }}$ is obtained by the chemical potential method in conjunction with SCFT.

the chemical potential is large at the interface, a small umbrella potential cannot constrain the system. In some circumstance (e.g., the alchemical transformation [96]), we need to investigate highly unstable state with a large chemical potential which requires a large umbrella potential constraint.

In the limit of $\lambda N \rightarrow \infty$, we have,

$$
\mu_{\lambda N}\left[m_{\text {target }}\right](x)=\mu_{\text {exact }}\left[m_{\text {target }}\right](x)
$$

As shown in the Fig. 3.3, the difference between the $\mu_{\text {exact }}\left[m_{\text {target }}\right](x)$ and the $\mu_{\lambda N}\left[m_{\text {target }}\right](x)$ becomes smaller, when we increase the strength of the umbrella potential, $\lambda N$. At $\lambda N=10000$, the maximum difference between $\mu_{\text {exact }}\left[m_{\text {target }}\right](x)$ and $\mu_{\lambda N}\left[m_{\text {target }}\right](x)$ is only approximately $1.23 \%$.

The deviation between $\mu_{\lambda N}\left[m_{\text {target }}\right](x)$ and $\mu_{\text {exact }}\left[m_{\text {target }}\right](x)$ is measured by calculating the free-energy difference, $\Delta \mathcal{F}$ between $\mathcal{F}_{\lambda}$ and $\mathcal{F}_{\text {exact }}$. In Fig. 3.4, we plot the $\Delta \mathcal{F}$ as a function of $\lambda N$. At large $\lambda N, \Delta \mathcal{F}$ decays with a power law 


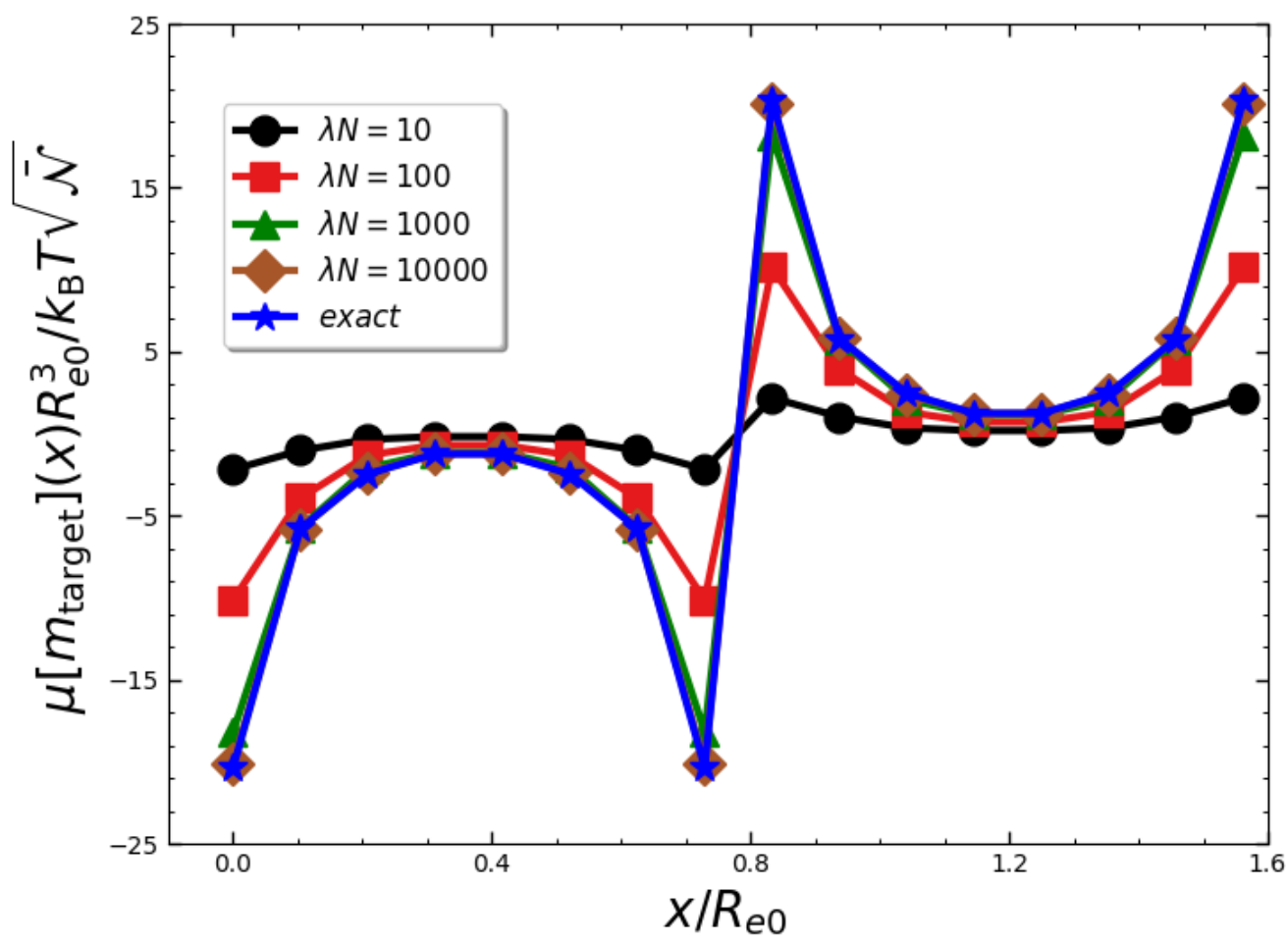

FIGURE 3.3: The chemical potential $\mu_{\lambda N}\left[m_{\text {target }}\right](x)$ of diblock copolymers is calculated with different values of $\lambda N$. The blue curve is the exact result obtained from the exact delta constraint method.

$\propto(\lambda N)^{-\eta}$. The decay exponent $\eta$ is related to the state $m$ and the form of the free-energy functional. In this case, we obtained an exponent $\eta \approx 0.92$.

From above discussions, we conclude that the umbrella potential method and the exact-delta constraint method are equivalent in the limit of large $\lambda N$. In principle, we can use both methods to calculate the free energy or the chemical potential for a specific state, $m$. Practically, we only use the umbrella potential method due to the computational simplicity.

\subsubsection{A comparison of the umbrella potential method in SCFT and the field-theoretic umbrella sampling method in conjunction with the SCMF algorithm}

One difference between a particle-based simulation and the SCFT method is that the particle-based simulation takes into account thermal fluctuations, which can be reduced by increasing $\overline{\mathcal{N}}$. The umbrella potential method in conjunction 


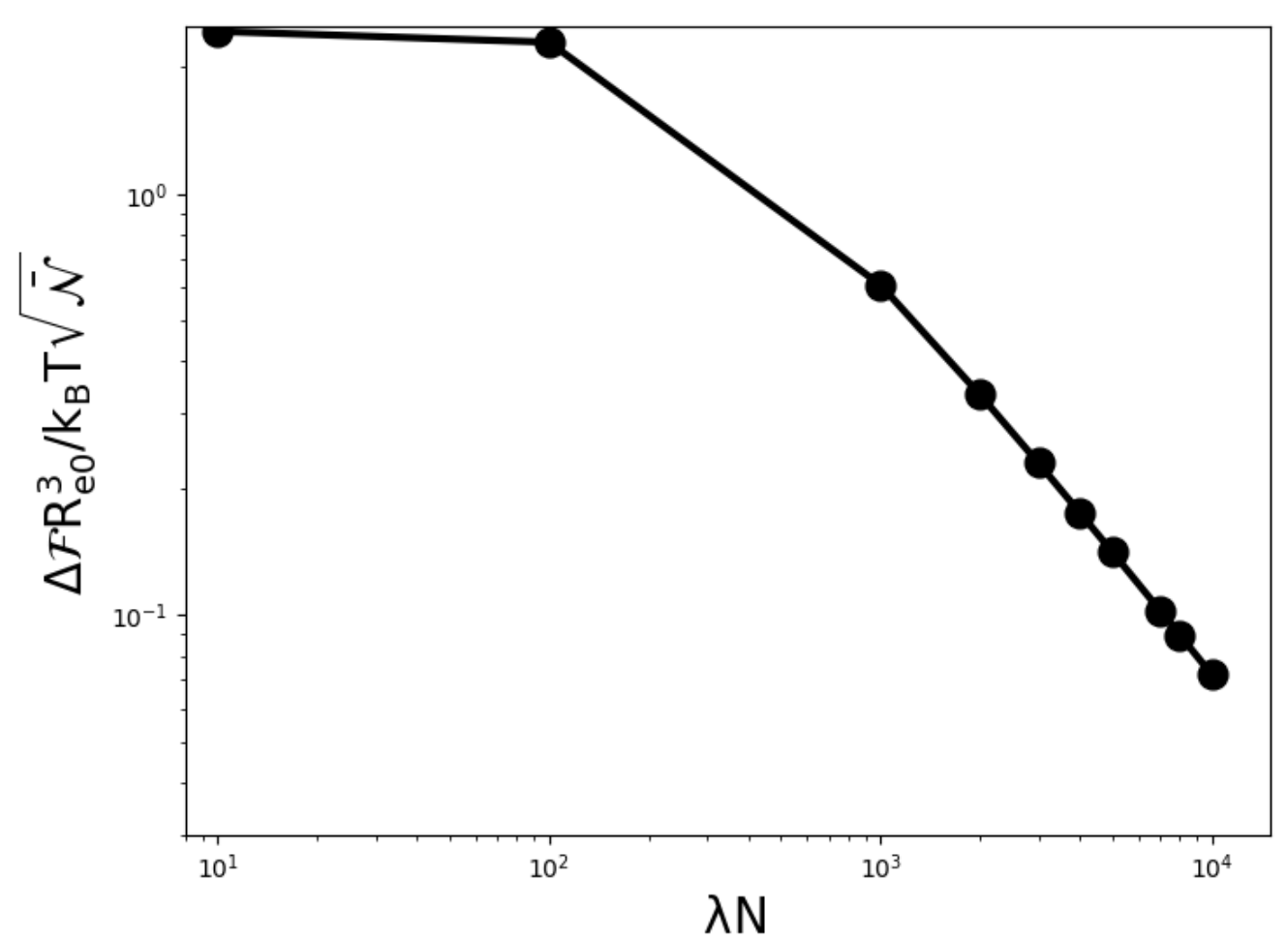

FIGURE 3.4: The free-energy difference $\Delta \mathcal{F}$ between $\mathcal{F}_{\lambda}$ and $\mathcal{F}_{\text {exact }}$ is calculated with different values of $\lambda N$.

with SCFT is accurate at large value of $\lambda N$. In the particle-based simulation, we cannot use a very large value of $\lambda N$ due to the poor sampling scheme. In this subsection, we will compare the umbrella potential method in SCFT to the field-theoretic umbrella sampling method in conjunction with the SCMF, particle-based simulation with a value of $\lambda N=50$.

To make a comparison with the particle-based simulation, we also calculate the chemical potential of the state, $m_{\text {target }}$. The size of the system along the x-axis is also set as $L_{x}=1.5 R_{\mathrm{e} 0}$. The discretization of the system, $\Delta x$ equals to $1 / 12 R_{\mathrm{e} 0}$. There are 12 grid points on $\mathrm{y}$ and $\mathrm{z}$ axis, respectively. The chain discretization in the SCMF algorithm is $N=32$.

We run the simulation with different values of $\overline{\mathcal{N}}$. As shown in Fig. 3.5, in a large value of $\overline{\mathcal{N}}=32768^{2}$, the deviation between the particle-based simulation and the SCFT is very small. In the limit of $\overline{\mathcal{N}} \rightarrow \infty$, the fluctuations in the SCMF are eliminated and becomes identical with the SCFT method. With a large value of $\lambda N$, thermal fluctuations can also be eliminated in the particle-based simulations. However, we cannot use a very large value of $\lambda N$ in the particle-based 


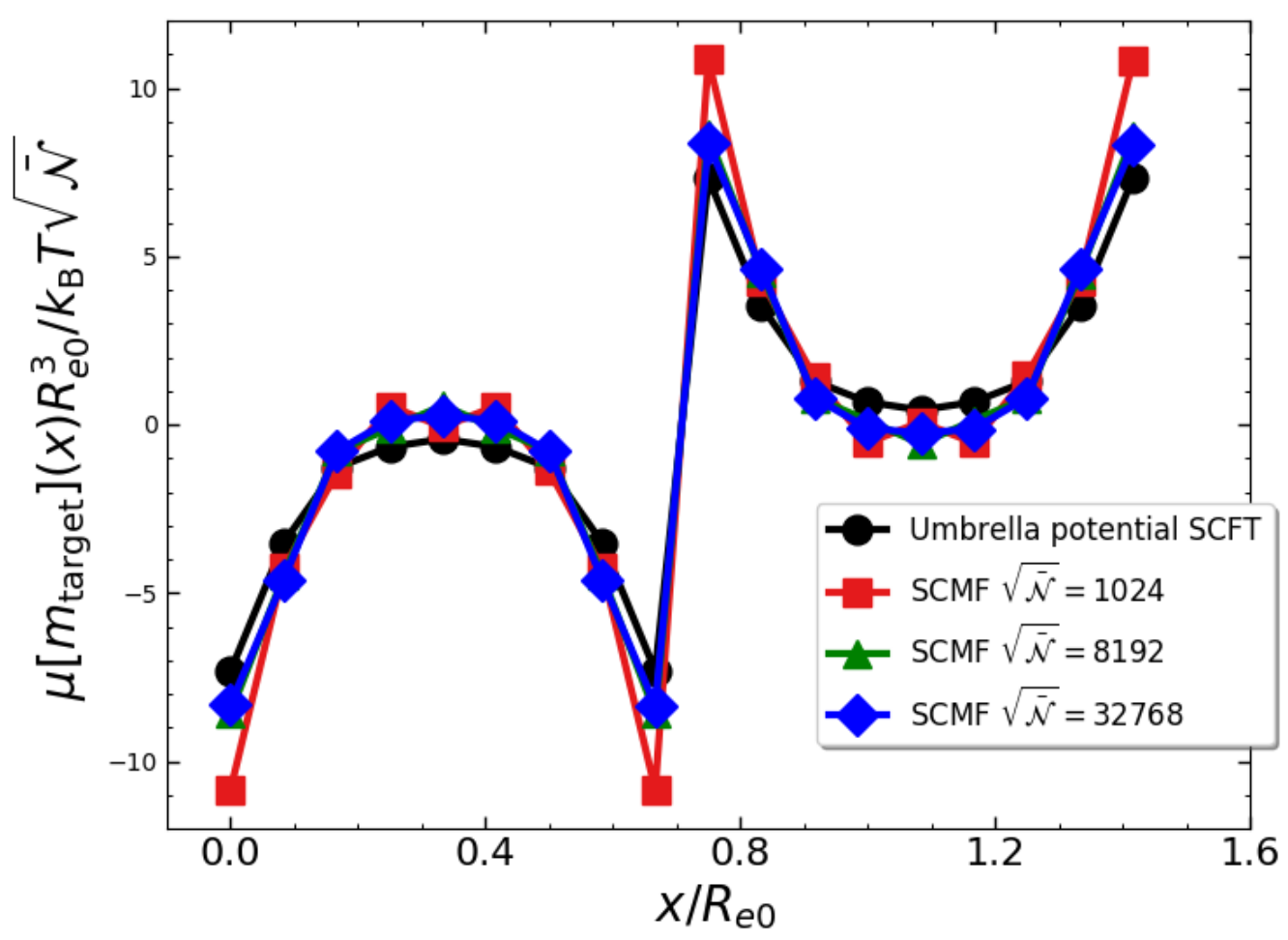

FIGURE 3.5: The chemical potential of diblock copolymers computed by the field-theoretic umbrella sampling method at $\lambda N=50$. The chemical potential $\mu_{\lambda N}\left[m_{\text {target }}\right](x)$ of diblock copolymers is calculated with different values of $\overline{\mathcal{N}}$ and compare the results with the SCFT.

simulations. Because the incompressible parameter, $\kappa N$ is fixed to 50 in the particle-based simulations. The energy contribution from the incompressible term may be negligibly small compared to the contribution from the umbrella potential. As a result, the system will probably violate the incompressible constraint.

From above discussions, we find that the umbrella potential method in SCFT and the field-theoretic umbrella sampling method in SCMF are equivalent, in the limit of $\overline{\mathcal{N}} \rightarrow \infty$. The advantage of the umbrella potential method in SCFT is that it can use a very large value of $\lambda N$, which enhances the accuracy of the chemical potential. 


\subsection{Dynamic evolution of densities}

A collective process in polymeric systems involves the co-operative movement of many molecules, which results in a molecular flow. One important approach to investigate collective phenomena is by directly evolving the particle's density instead of a particle-based simulation.

The driving force for the density evolution is the chemical potential. In the previous sections, we have introduced several numerical methods to compute the chemical potential, $\mu[m]$ for a target state, $m$. To construct a dynamic equation, the next step is to translate the thermodynamic driving force into a flow of particles via the Onsager coefficient, $\Lambda\left(\vec{r}, \vec{r}^{\prime}\right)$,

$$
\mathbf{J}(\vec{r})=-\int \mathrm{d} \vec{r}^{\prime} \Lambda\left(\vec{r}, \vec{r}^{\prime}\right) \nabla^{\prime} \mu\left[m\left(\vec{r}^{\prime}\right)\right] .
$$

A dynamic equation can be constructed by a combination with the continuity equation.

$$
\nabla \cdot \mathbf{J}(\vec{r})+\frac{\partial m}{\partial t}=0
$$

As a result, the dynamic density evolution equation takes the form of model B [97],

$$
\frac{\partial m(\vec{r})}{\partial t}=\nabla \cdot \int \mathrm{d} \vec{r}^{\prime} \Lambda\left(\vec{r}, \vec{r}^{\prime}\right) \nabla^{\prime} \mu\left[m\left(\vec{r}^{\prime}\right)\right]
$$

For simple liquids, which are made up of simple small units, dynamic evolution equations are proved to be valid by a comparison with molecular dynamics simulations $[98,99]$. In the case of polymeric systems, the connectivity of the polymer chain makes the problem complicated. Because the thermodynamic driving forces are transmitted along the molecular backbone, the Onsager coefficient becomes nonlocal in space.

In the following subsections, we will discuss the dynamics of density evolution for diblock copolymers, based on the mean-field, SCFT method, and the particle-based, SCMF algorithm. 


\subsubsection{Conserved dynamics}

To construct a dynamic evolution equation for diblock copolymers, it is crucial to obtain the Onsager coefficient. If we do not take into account the influence of the molecular connectivity, we will obtain an Onsager coefficient which is local in space. In an incompressible system, the Onsager coefficient takes the form [100],

$$
\Lambda_{\text {local }}\left(\vec{r}, \vec{r}^{\prime}\right)=\frac{D_{\text {Rouse }}}{4}\left[1-m(\vec{r})^{2}\right] \delta\left(\vec{r}-\vec{r}^{\prime}\right),
$$

where $D_{\text {Rouse }}$ is the single-chain diffusion constant in Rouse dynamics. This Ansatz is called "local dynamics". In diblock copolymer melts, the collective motion of diblock copolymers is described by the composition, i.e., the order parameter $m=\phi_{A}-\phi_{B}$. The term $\left[1-m(\vec{r})^{2}\right]$ accounts for the incompressibility of the polymer blends. This term assumes that the flow of A monomers will generate a reverse flow of $\mathrm{B}$ monomers at the same time.

If the impact of the connectivity of the molecular backbone is taken into account, the Onsager coefficient is nonlocal. The thermodynamic forces will be transmitted along the molecular backbones. A nonlocal coupling Ansatz is proposed [101],

$$
\Lambda_{\alpha, \beta}\left(\vec{r}, \vec{r}^{\prime}\right)=\Lambda_{\text {local }}\left(\vec{r}, \vec{r}^{\prime}\right) P_{\alpha, \beta}\left(\vec{r}, \vec{r}^{\prime}\right) .
$$

Here, $P_{\alpha, \beta}\left(\vec{r}, \vec{r}^{\prime}\right)$ is the pair density of monomers $\alpha, \beta$ from the same chain at positions $\vec{r}$ and $\overrightarrow{r^{\prime}}$. In practice, the calculation of $P_{\alpha, \beta}\left(\vec{r}, \vec{r}^{\prime}\right)$ is not straightforward. Further approximations are made for a linear homopolymer chain. One employ that Debye correlation function, $g_{0}\left(\vec{r}, r^{\prime}\right)$. This Ansatz is also called the "Debye dynamics".

The Debye dynamics Ansatz is used to investigate collective phenomena, e.g., the spinodal decomposition process of block copolymers [22, 102, 103].

\subsubsection{External potential dynamics (EPD)}

In the previous section, we introduced dynamic evolution equations to evolve the densities of block copolymer system. Alternatively, it is also possible to 
evolve auxiliary fields which are in conjunction with the densities in the framework of the SCFT method. This numerical scheme is called the "external potential dynamics" [22].

One can rewrite the Hamiltonian in terms of independent chains in fluctuating fields. Then, one can integrate out the particles' coordinates and obtain a Hamiltonian which only depends on the auxiliary fields. By assuming that the Onsager coefficient is translationally invariant, one can evolve the auxiliary fields to mimic a polymer dynamics in a real system.

We start with the partition function $\mathcal{Z}_{\mathrm{EPD}}$, which is equivalent to the $\mathcal{Z}_{\mathrm{sct}}$ in Eq. 2.51,

$$
\begin{aligned}
\mathcal{Z}_{\mathrm{EPD}} \sim & \int \prod_{i=1}^{n} \prod_{s=0}^{N_{s}} \mathrm{~d} \vec{r}_{i}(s) \exp \left[-\hat{\mathcal{H}}_{0} / k_{\mathrm{B}} T\right] \\
& \exp \left\{-\left[\int \mathrm{d} \vec{r}-\frac{\chi \rho}{4}\left(\hat{\phi}_{A}-\hat{\phi}_{B}\right)^{2}+\ln \delta\left(1-\hat{\phi}_{A}-\hat{\phi}_{B}\right)\right]\right\}
\end{aligned}
$$

The external potential $W_{A}$ and $W_{B}$ are replaced by,

$$
\begin{aligned}
& W_{A}=\frac{U+W}{2} \\
& W_{B}=\frac{U-W}{2} .
\end{aligned}
$$

By using the HS transformation, the partition function is modified as,

$$
\begin{aligned}
\mathcal{Z}_{\mathrm{EPD}} \sim & \int \prod_{i=1}^{n} \prod_{s=0}^{N_{s}} \mathrm{~d} \vec{r}_{i}(s) \exp \left[-\hat{\mathcal{H}}_{0} / k_{\mathrm{B}} T\right] \\
& \int \mathcal{D} W \exp \left\{-\frac{\rho}{N} \int \mathrm{d} \vec{r}\left(\hat{\phi}_{A}-\hat{\phi}_{B}\right) \frac{W}{2}+\frac{W^{2}}{4 \chi N}\right\} \\
& \int \mathcal{D} U \exp \left\{-\frac{\rho}{N} \int \mathrm{d} \vec{r} \frac{U}{2}\left(\hat{\phi}_{A}+\hat{\phi}_{B}-1\right)\right\}
\end{aligned}
$$

We can define a free-energy functional, $G[U, W]$ in terms of the external fields, $W$ and $U$. 


$$
\mathcal{Z}_{\mathrm{EPD}} \sim \int \mathcal{D} W \mathcal{D} U \exp \left\{-G[U, W] / k_{\mathrm{B}} T\right\}
$$

where,

$$
\frac{G[U, W]}{n k_{\mathrm{B}} T} \equiv-\ln \mathcal{Q}[U, W]+\frac{1}{V} \int \mathrm{d} \vec{r}\left\{\frac{W^{2}}{4 \chi N}-\frac{U}{2}\right\}
$$

The single-chain partition function $\mathcal{Q}[U, W]$ is defined as,

$$
\begin{aligned}
\mathcal{Q}[U, W]= & \int \prod_{s=0}^{N_{s}} \mathrm{~d} \vec{r}(s) \exp \left[-\hat{\mathcal{H}}_{b} / k_{\mathrm{B}} T\right] \\
& \exp \left\{-\frac{\rho}{N}\left(\int \mathrm{d} \vec{r}\left(\hat{\phi}_{A 1}-\hat{\phi}_{B 1}\right) \frac{W}{2}+\left(\hat{\phi}_{A 1}+\hat{\phi}_{B 1}\right) \frac{U}{2}\right)\right\} .
\end{aligned}
$$

where $\hat{\phi}_{A 1}$ and $\hat{\phi}_{B 1}$ are segment density functions of a single chain.

$$
\begin{aligned}
\hat{\phi}_{A 1}(\vec{r}) & =\frac{1}{\rho} \sum_{s=0}^{N_{A}} \delta[\vec{r}-\vec{r}(s)] . \\
\hat{\phi}_{B 1}(\vec{r}) & =\frac{1}{\rho} \sum_{s=N_{A}}^{N_{s}} \delta[\vec{r}-\vec{r}(s)] .
\end{aligned}
$$

The functional derivative of the free-energy functional, $G[U, W]$ with respect to $W$ is,

$$
\frac{V}{n k_{\mathrm{B}} T} \frac{\delta G[U, W]}{\delta W}=-\frac{V}{\mathcal{Q}[U, W]} \frac{\delta \mathcal{Q}[U, W]}{\delta W}+\frac{1}{2 \chi N} W=-\left(\Phi_{A}-\Phi_{B}\right)+\frac{1}{2 \chi N} W .
$$

$\Phi_{A}$ and $\Phi_{B}$ are densities that are conjugated to the fields, $W_{A}$ and $W_{B}$, respectively.

The functional derivative of $G[U, W]$ with respect to the field $U$ gives,

$$
\frac{V}{n k_{\mathrm{B}} T} \frac{\delta G[U, W]}{\delta U}=\Phi_{A}+\Phi_{B}=1
$$

We consider an incompressible system which enforces the free-energy functional $G[U, W]$ depending only on the field $W$. Given an external potential, $W$, we can calculate potential field, $U$ by the saddle point approximation. We 
use an iterative procedure to obtain the external potential $U$ which satisfy the equation 3.55 ,

$$
U^{n+1}=U^{n}+\Delta t\left(\Phi_{A}(\vec{r})^{n}+\Phi_{B}(\vec{r})^{n}-1\right)
$$

where $\Delta t$ is the step length in each iteration.

The calculation can be accelerated by considering the structure factor of diblock copolymers. The iterative equation 3.56 is modified as [92],

$$
\begin{aligned}
& U^{n+1}(\mathbf{q} \neq 0)=U^{n}(\mathbf{q})+\left(\Phi_{A}^{n}(\mathbf{q})+\Phi_{B}^{n}(\mathbf{q})-1\right) \frac{\Delta t}{1+\Delta t S(\mathbf{q})} \\
& U^{n+1}(\mathbf{q}=0)=0
\end{aligned}
$$

$S(\mathbf{q})$ is the composition structure factor which is obtained in the Appendix B.

To investigate the time evolution of the composition, $m$ which is conjugated to the order parameter, $W$, we consider the conserved dynamics of $W$ is given by model B,

$$
\frac{\partial W(r)}{\partial t}=\nabla_{\vec{r}^{\prime}} \int \mathrm{d} \vec{r}^{\prime} \Lambda\left(\vec{r}, \vec{r}^{\prime}\right) \nabla_{\vec{r}^{\prime}} \frac{\delta G[U, W]\left(\vec{r}^{\prime}\right)}{\delta U\left(\vec{r}^{\prime}\right)}
$$

In the end, we summarize the main procedures of the EPD method. We initially have the external fields $W$. Then we generate the corresponding fields $U$ by the Eq. 3.57. By evolving $W$ by the Eq. 3.59, we can generate new external fields $W$. The biggest advantage of the EPD method is that it is magnitude faster compared to the density evolution method in the SCFT. Because EPD method only needs to evolve the field $W$. Comparably, the DSCFT method needs to find two fields $W_{A}$ and $W_{B}$ from the density $\Phi_{A}$ and $\Phi_{B}$. This method is widely applied to investigate the structure formation process in polymer blends and solutions $[102,104]$.

\subsection{A direct measurement of Onsager coefficients in particle-based simulations}

Theoretical studies on the structure formation of block copolymers are of interests for both theoretical investigations and the industry [105-107]. To realize 
a large time and length scale simulation, a field-theoretic, continuum model is a necessity [54]. The degrees of freedom of the particles' coordinates are integrated out but the information of the molecular connectivity can be preserved. For example, the self-consistent field theory (SCFT) has been successfully applied to mapped out the phase diagram of various block copolymers in equilibrium $[17,18,108]$.

In order to use a continuum description to investigate the dynamical or kinetical properties of block copolymers, the first procedure is to pick up an "appropriate" order parameter to formulate the correspondent free-energy functional. For example, one usually selects the local densities of different components as the order parameter. In many circumstances (e.g., the macroscopic phase separation in polymer blends), this order parameter is a slow variable compared to the relaxation time of a polymer chain. We can assume that the chain conformation is always equilibrated during the density evolution [64, 68, 101, 109-111]. In such circumstances, we can use a purely dynamic equation, which employs the local composition, $m(\mathbf{r})$ in a binary homopolymer blend, as the order parameter to investigate the early stages of phase separation $[22,27,48,103,112-$ 117]. This assumption is valid in many circumstances especially in intrinsically slow collective processes (e.g., the Lifshitz-Slyozov coarsening in the binary homopolymer blends [118]). A multiple-time integrator method which evolves the densities and the molecular configuration separately by combining the SCMF algorithm simulation and the continuum Flory-Huggins-de Gennes model has been devised to accelerate the particle-based simulations [110]. In the end, the speedup of this scheme reaches two orders of magnitudes compared to a purely particle-based simulation. This assumption is not available when we consider a relaxation process from a highly non-equilibrium, stretched state (i.e., the chain which is under tension), the interplay of the structure formation and the relaxation of the polymer chain are not negligible. Researchers find that the planar elongation of the molecular conformation of symmetric diblock copolymer melts in a disordered initial state will result in an alignment of lamella normals perpendicular to the stretch direction during the self-assembly process [57].

The next procedure in a continuum description is to evolve the order parameter. In this section, we consider the case that employs collective variables, i.e., the local densities as the order parameter. The co-operative movement of 
molecules is related to the single-chain dynamics by the Onsager coefficient, $\Lambda\left(\mathbf{r}, \mathbf{r}^{\prime}\right)$. The Onsager coefficient, $\Lambda\left(\mathbf{r}, \mathbf{r}^{\prime}\right)$ translates thermodynamic forces into a flow of particles. In the simplest case for a polymer chain with Rouse dynamics, the Onsager coefficient, $\Lambda\left(\mathbf{r}, \mathbf{r}^{\prime}\right)$ is characterized by the single-chain correlation function $g\left(\mathbf{r}, \mathbf{r}^{\prime}\right)$. One obtains,

$$
\Lambda\left(\mathbf{r}, \mathbf{r}^{\prime}\right)=\frac{N D}{\rho k_{\mathrm{B}} T}\left(1-m^{2}\right) \frac{g\left(\mathbf{r}, \mathbf{r}^{\prime}\right)}{V}
$$

Here $D$ is the self-diffusion constant of a polymer chain. $\mathrm{N}$ is the degree of polymerization and $V$ is the volume of the system [54, 101, 119].

In this section, we investigate the interplay between the single-chain dynamics and the dynamics of collective variables by directly measuring the Onsager coefficient with a particle-based simulation. We implement the particle-based simulation by means of the SCMF algorithm, which retains the Rouse dynamics.

We find that the Onsager coefficient is related to the single-chain dynamic structure factor, $g_{s}$. As a result, we assume that the Onsager coefficient, which gives rise to a nonlocal term (e.g., the Debye function in binary homopolymer blends) also is time-dependent to account for the subdiffusive segmental dynamics at short times.

In the following, we will introduce a numerical scheme to measure the Onsager coefficient in a quasi-one-dimensional system. We find that our numerical results are in agreement with analytical predictions [120]. Additionally, this method is rather general and can be easily extended to more complicated systems. By measuring the Onsager coefficient, we find that monomers move independently at the very initial stage. The thermodynamic forces are not transferred along the polymer backbone. By increasing the simulation time, the evolution of the densities gradually mimics the prediction in Eq. 3.60. This theory assumes that all the monomers of a chain move coherently on large time and length scale. 


\subsubsection{Onsager coefficient: the long-time limit}

In this section, we will derive an analytical form of the Onsager coefficient in a symmetric binary homopolymer blend for the long-time limit.

We assume that the Onsager coefficient is time-dependent and take into account the memory effect. The Onsager coefficient, $\Lambda\left(\mathbf{r}, \mathbf{r}^{\prime}, t, t^{\prime}\right)$ connects the thermodynamic force to the order-parameter flow, $\mathbf{J}(\mathbf{r})$ [121-123]:

$$
\mathbf{J}(\mathbf{r}, t)=-\int_{t^{\prime}=0} \mathrm{~d} t^{\prime} \int \mathrm{d} \mathbf{r}^{\prime} \Lambda\left(\mathbf{r}, \mathbf{r}^{\prime}, t, t^{\prime}\right) \nabla^{\prime} \mu[m]\left(\mathbf{r}^{\prime}, t^{\prime}\right) .
$$

The dynamic equation of the composition can be formulated by the continuity equation,

$$
\frac{\partial m(\mathbf{r}, t)}{\partial t}=-\nabla \cdot \mathbf{J}(\mathbf{r}, t)
$$

In the end, the dynamic equation to evolve the order-parameter takes the form,

$$
\frac{\partial m(\mathbf{r}, t)}{\partial t}=\nabla \int \mathrm{d} t^{\prime} \int \mathrm{d} \mathbf{r}^{\prime} \Lambda\left(\mathbf{r}, \mathbf{r}^{\prime}, t, t^{\prime}\right) \nabla^{\prime} \mu\left(\mathbf{r}^{\prime}, t^{\prime}\right) .
$$

We assume that the Onsager coefficient is translationally invariant in time and space, i.e., $\Lambda\left(\mathbf{r}, \mathbf{r}^{\prime}, t, t^{\prime}\right)=\Lambda\left(\mathbf{r}-\mathbf{r}^{\prime}, t-t^{\prime}\right) \cdot m(\mathbf{q}, \omega), \Lambda(\mathbf{q}, \omega)$, and $\mu(\mathbf{q}, \omega)$ represents the Fourier transform $m, \Lambda$, and $\mu$, respectively.

The dynamic equation in the reciprocal space is derived as,

$$
-m(\mathbf{q}, 0)-i \omega m(\mathbf{q}, \omega)=-\mathbf{q}^{2} \Lambda(\mathbf{q}, \omega) \mu(\mathbf{q}, \omega)
$$

Different Fourier modes are decoupled in the reciprocal space. The Onsager coefficient is therefore derived as,

$$
\Lambda(\mathbf{q}, \omega)=\frac{1}{\mathbf{q}^{2}} \frac{m(\mathbf{q}, t=0)+i \omega m(\mathbf{q}, \omega)}{\mu(\mathbf{q}, \omega)} .
$$

In the following, we will derive the Onsager coefficient in a binary homopolymer mixture, which contains $n_{A}=n / 2$ A polymers and $n_{B}=n / 2 \mathrm{~B}$ polymers in a volume $V$, respectively. The incompatible parameter, $\chi N$ is determined as 
0 , which favors mixing in equilibrium. We employ the local composition of the two different species as the order parameter, i.e., $m=\phi_{A}-\phi_{B}$.

The chemical potential of a target state, $m$ is obtained by the Eq. 3.3,

$$
\frac{\mu_{\text {blends }}^{\mathrm{RPA}}[m] R_{e 0}^{3}}{k_{\mathrm{B}} T \sqrt{\overline{\mathcal{N}}}}=\frac{R_{\mathrm{e} 0}^{3}}{\sqrt{\overline{\mathcal{N}}}} \frac{\delta \mathcal{F}_{\text {blend }}^{\mathrm{RPA}}[m]}{k_{\mathrm{B}} T \delta m}=\left[m-\frac{R_{\mathrm{e} 0}^{2} \Delta m}{18}\right] .
$$

We consider a decay process in a one-dimensional system, $V=L \times R_{\mathrm{e} 0} \times R_{\mathrm{e} 0}$. The initial density profile, $m(x, 0)=A_{0} \sin (q x)$ consists only one Fourier mode q. $A_{0}$ is the amplitude of the density profile. The density profile at time $t$ is represented as, $m(x, t)$. When $t \rightarrow \infty$, we have $m(x, t)=0$.

There are three reasons for us to chose this particular condition: if the initial composition variation is shallow, i.e., $A_{0} \ll 1$, (i) the chemical potential can be accurately and analytically obtained by the RPA, (ii) the chain conformations are Gaussian because they are not distorted by the weak spatial inhomogeneity, (iii) our study focus on the time-dependence of the Onsager coefficient. Since there is only one single spatial Fourier mode involved, deviations from the long-time behavior cannot be captured by fiddling with the q-dependence of $\Lambda$ [124].

We observe the relaxation process from the initial density profile $m(x, 0)$ to the equilibrium homogeneous state. The density profile will decrease its amplitude and keeps the wave factor $q$ unchanged. The time evolution of the density profile, $m(x, t)$ satisfies $m(x, t)=A(q, t) \sin (q x)$.

We use the symbol $\mathcal{F}_{t}$ and $\mathcal{F}_{x}$ to represent the Fourier transform in time and space. $m(q, \omega)$ takes the form,

$$
m(q, \omega)=\mathcal{F}_{t}[A(q, t)] \mathcal{F}_{x}[\sin (q x)]=A(q, \omega) \mathcal{F}_{x}[\sin (q x)]
$$

with $A(q, \omega) \equiv \mathcal{F}_{t}[A(q, t)]$.

According to the Eq. 3.66, the time evolution of the chemical potential, $\mu_{\text {blend }}^{\mathrm{RPA}}[m](x, t)$ by the RPA is obtained as,

$$
\frac{R_{\mathrm{e} 0}^{3} \mu_{\mathrm{blend}}^{\mathrm{RPA}}[m](x, t)}{\sqrt{\mathcal{N}} k_{\mathrm{B}} T}=A(q, t) \sin (q x)\left(1+\frac{R_{\mathrm{e} 0}^{2} q^{2}}{18}\right) .
$$


The Onsager coefficient by the RPA is represented as,

$$
\Lambda_{\text {blend }}^{\mathrm{RPA}}(q, \omega)=\frac{1}{q^{2}\left(1+q^{2} R_{\mathrm{e} 0}^{2} / 18\right)} \frac{A_{0}+i \omega A(q, \omega)}{A(q, \omega)} \frac{R_{\mathrm{e} 0}^{3}}{k_{\mathrm{B}} T \sqrt{\overline{\mathcal{N}}}} .
$$

In order to obtain an analytical form of the Onsager coefficient, we need to obtain the time evolution of the densities, i.e., the $A(q, \omega)$. The collective motion of the monomers is related to the single-chain dynamics of a polymer chain. We use the symbol $\vec{r}_{i}(s)$ to represent the position of the $s^{\text {th }}$ segment on polymer $i$. We have the following approximate RPA-like relation between the collective dynamical structure factor and the single-chain dynamic structure factor,

$$
\begin{aligned}
& \left\langle m(\mathbf{q}, t) m\left(-\mathbf{q}, t_{0}\right)\right\rangle \\
= & \frac{1}{\rho^{2}} \sum_{i^{\prime} i} \sum_{s, s^{\prime}} \gamma\left(i, i^{\prime}\right)\left\langle e^{-i \mathbf{q} \vec{r}_{i}(s, t)} e^{i \mathbf{q} \vec{q}_{i^{\prime}}\left(s^{\prime}, t_{0}\right)}\right\rangle \\
\approx & \frac{N}{\rho^{2}} \sum_{i} \sum_{s, s^{\prime}} \frac{1}{N}\left\langle e^{-i \mathbf{q} \vec{r}_{i}(s, t)+i \mathbf{q} \vec{r}_{i}\left(s^{\prime}, t_{0}\right)}\right\rangle-\frac{2}{\rho^{2}} \sum_{i \in A} \sum_{i^{\prime} \in B} \sum_{s, s^{\prime}}\left\langle e^{-i \mathbf{q} \vec{r}_{i}(s, t)}\right\rangle\left\langle e^{i \mathbf{q}_{i^{\prime}}\left(s^{\prime}, t_{0}\right)}\right\rangle \\
& +\frac{1}{\rho^{2}} \sum_{i \in A} \sum_{i^{\prime} \in A, i \neq i^{\prime}} \sum_{s, s^{\prime}}\left\langle e^{-i \mathbf{q} \vec{r}_{i}(s, t)}\right\rangle\left\langle e^{i \mathbf{q}_{i^{\prime}}\left(s^{\prime}, t_{0}\right)}\right\rangle+\frac{1}{\rho^{2}} \sum_{i \in B} \sum_{i^{\prime} \in B, i \neq i^{\prime}} \sum_{s, s^{\prime}}\left\langle e^{-i \mathbf{q}_{i}(s, t)}\right\rangle\left\langle e^{i \mathbf{q}_{i_{i}^{\prime}}\left(s^{\prime}, t_{0}\right)}\right\rangle \\
\approx & \frac{N}{\rho^{2}} \sum_{i} \sum_{s, s^{\prime}} \frac{1}{N}\left\langle e^{-i \mathbf{q} \vec{r}_{i}(s, t)+i \mathbf{q} \vec{r}_{i^{\prime}}\left(s^{\prime}, t_{0}\right)}\right\rangle \\
= & \frac{n N}{\rho^{2}} g_{s}(\mathbf{q}, t),
\end{aligned}
$$

where $\gamma\left(i, i^{\prime}\right)=-1$, when $i, i^{\prime}$ belongs to different type of polymers, otherwise $\gamma\left(i, i^{\prime}\right)=1$ and $t_{0}$ is at $t=0$. The $\langle\ldots\rangle$ represents the ensemble average over particles' configurations. The interactions between monomers on different polymer chains are dominated by the forces to suppress the total density fluctuations. These interactions are rather weak, so we neglect the inter-chain correlations.

$g_{s}(\mathbf{q}, t)$ is the single-chain dynamic structure factor and is derived as [56], 


$$
\begin{aligned}
g_{s}(\mathbf{q}, t)= & \frac{1}{N} \sum_{s, s^{\prime}} \exp \left\{-\frac{\left(\mathbf{q} R_{\mathrm{e} 0}\right)^{2}\left|s-s^{\prime}\right|}{6 N}-D \mathbf{q}^{2} t\right. \\
- & \frac{2\left(\mathbf{q} R_{e 0}\right)^{2}}{3 \pi^{2}} \sum_{p=1} \frac{1}{p^{2}} \cos \left(\frac{\pi p s}{N}\right) \cos \left(\frac{\pi p s^{\prime}}{N}\right) \\
& \left.\left(1-e^{-3 \pi^{2} p^{2} t / \tau}\right)\right\} .
\end{aligned}
$$

The time evolution of the amplitude, $A(q, t)$ satisfies,

$$
A(q, t)=A_{0} \frac{g_{s}(q, t)}{g_{s}(q, 0)} .
$$

At large time and the small scattering angle $\left|\mathbf{q} R_{\mathrm{e} 0}\right| \ll 1$, the time evolution of $A(q, t)$ scales as $A_{0} \exp \left(-D q^{2} t\right)$. In experiments, this can be used to measure the diffusion constant, $D$. In this limit, the time evolution of the density profile satisfies $m(x, t)=A_{0} \exp \left(-D q^{2} t\right) \sin (q x)$.

In this limit $\omega \rightarrow 0$, the Onsager coefficient is,

$$
\Lambda_{\text {blend }}^{\mathrm{RPA}}(q, \omega)=\frac{D}{1+\left(q R_{\mathrm{e} 0}\right)^{2} / 18} \frac{R_{\mathrm{e} 0}^{3}}{k_{\mathrm{B}} T \sqrt{\mathcal{N}}} .
$$

This result is consistent with the Debey function Ansatz in Eq. 3.60. The term $1 /\left(1+\left(q R_{\mathrm{e} 0}\right)^{2}\right)$ is the Debey function, $S(q)$. This formula misses the term (1$\left.\mathrm{m}^{2}\right) / 4$, which accounts for coupling of the chain motion via the incompressibility constraint, i.e., the motion of different chains is uncorrelated.

\subsubsection{Evidence of time-dependent behavior for the Onsager co- efficient: numerical results in the high-frequency regime}

In the high-frequency regime (i.e., the time scale which is much smaller than the relaxation time of a polymer chain), the motion of the segments is subdiffusive. We assume that this factor will change the behavior of the Onsager coefficient from its long-time behavior. 


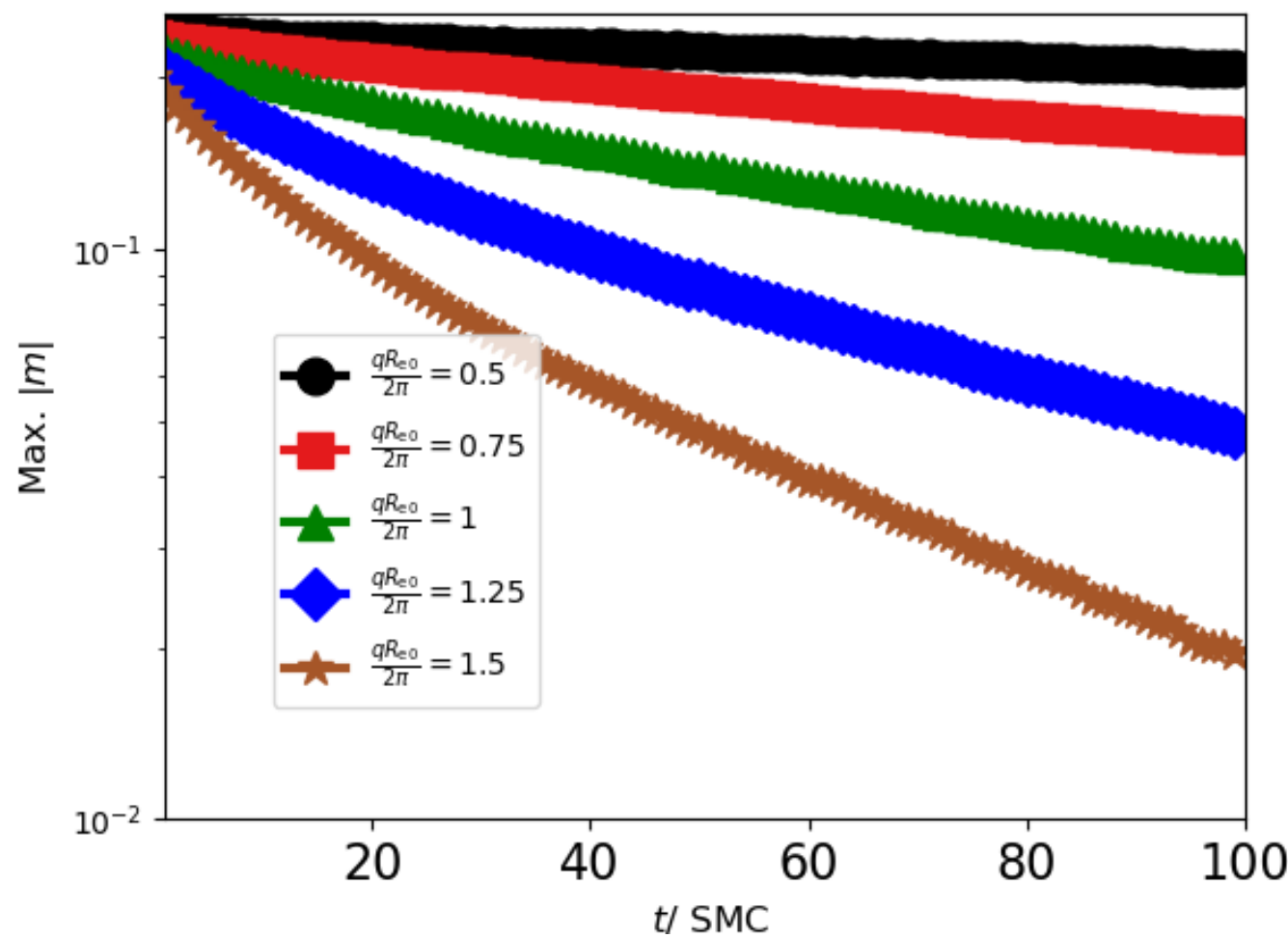

FIGURE 3.6: The amplitude of the density profile $A(q, t)$ is plotted as a function of the simulations time, $t$ with different Fourier modes, $q R_{\mathrm{e} 0} / 2 \pi$.

To confirm this assumption, we measure the time-dependent behavior of $A(q, t)$ in the particle-based simulation. We consider a decay process in a quasi-onedimensional system of volume, $V=L \times R_{\mathrm{e} 0} \times R_{\mathrm{e} 0}$ with a periodical boundary condition. We can average the density profile over the $y$ - and $z$-axis and consider only the variation of the density profile along the $x$-axis. The length of the system along the $\mathrm{x}$-direction $L$ is set to $8.0 R_{\mathrm{e} 0}$.

We restrain the system to the density profile $m(q, 0)$ with different Fourier modes $q$ by using an umbrella potential in the particle-based simulations. As illustrated in the previous section, the target density profile is a sine function $m(x, 0)=$ $A_{0} \sin (q x)$. Here $A_{0}$ is the amplitude which equals to 0.25 at $t=0$. The spatial discretization is $1 / 12 R_{\mathrm{e} 0}$, which limits the Fourier mode $q L / 2 \pi$ ranging from 1 to 12 . The discretization of the polymer chain is $N=32$, which concomitants a Rouse time $\tau_{\mathrm{R}} \approx 300$ SMCs (see Sec. 2.3.4.1).

After obtaining the target density profile $m(x, 0)$ in the particle-based simulation, we remove the umbrella potential and observe the time evolution of the 


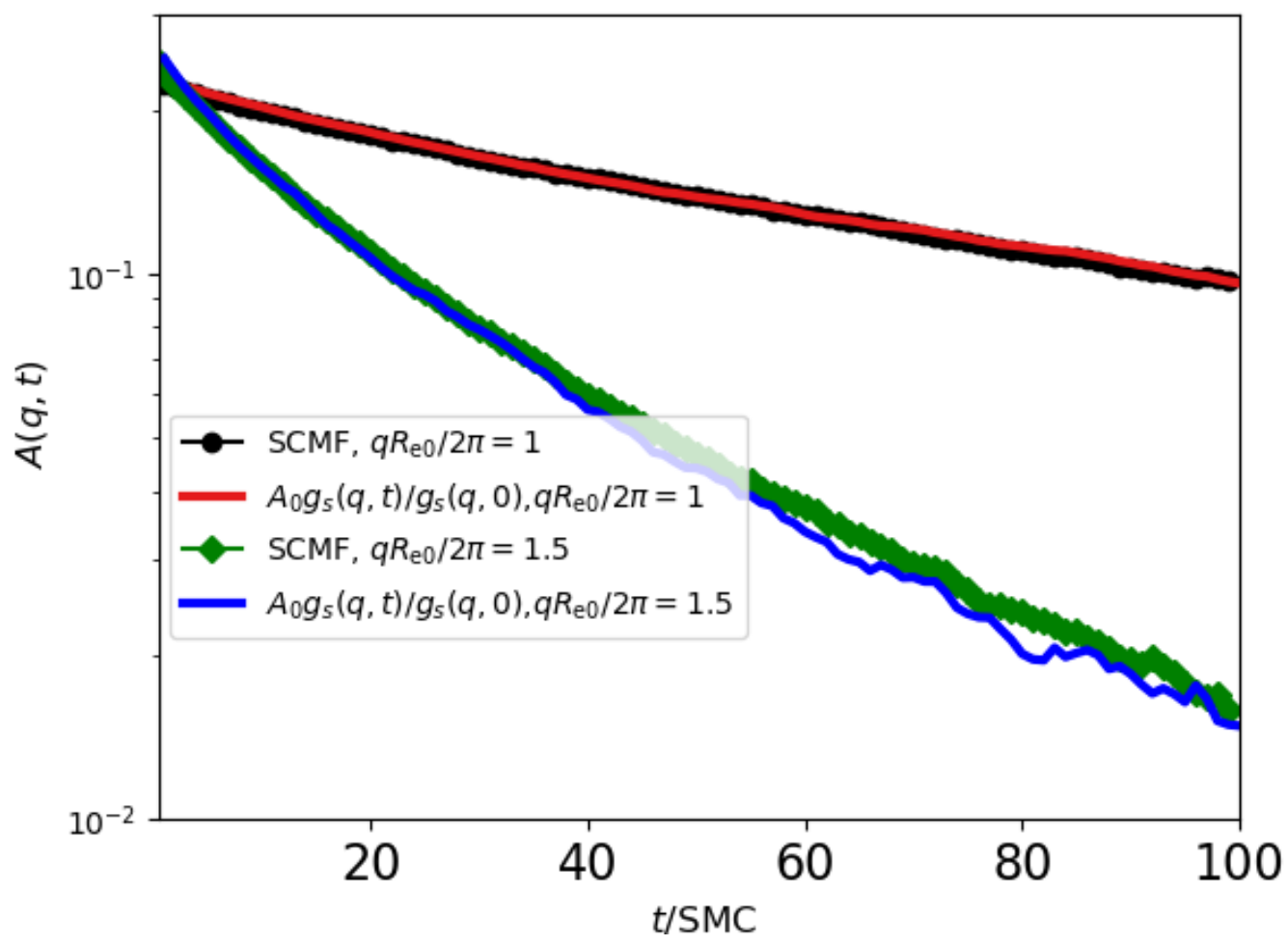

FIGURE 3.7: The time-evolution of density profile amplitude, $A(q, t)$ at $q R_{\mathrm{e} 0} / 2 \pi=1$ and $q R_{\mathrm{e} 0} / 2 \pi=1.5$, respectively. The results are well matched to the time-evolution of the single-chain, dynamic structure factor, $g_{s}(q)$.

density profile. We run the particle-based simulations for 100 SMCs. As expected, we find that the density profile only decreases its amplitude and keeps the wave factor $q$ unchanged.

In Fig. 3.6, we plot the averaged maximum absolute value of the density profile, i.e., $A(q, t)$ as a function of the simulation time, $t$. The density profile with a large value of $q$ decays fast in the simulation. At $q R_{\mathrm{e} 0} / 2 \pi=1.5$, the $A(q, t)$ decays approximately to 0.02 after 100 SMCs.

In the previous section, we predict that the time evolution of $A(q, t)$ satisfies $A(q, t)=A_{0} g_{s}(q, t) / g_{s}(q, 0)$. As shown in Fig. 3.7, we compare the time-evolution of the dynamic single-chain structure to the amplitude of the density, $A(q, t)$ at $q R_{\mathrm{e} 0} / 2 \pi=1$ and $q R_{\mathrm{e} 0} / 2 \pi=1.5$, respectively.

As a result, the Onsager coefficient is obtained as,

$$
\Lambda_{\mathrm{RPA}}(q, \omega)=\frac{1}{q^{2}} \frac{1}{1+\left(R_{\mathrm{e} 0} q\right)^{2} / 18}\left\{\frac{g_{s}(q, t=0)+i \omega g_{s}(q, \omega)}{g_{s}(q, \omega)}\right\} \frac{R_{\mathrm{e} 0}^{3}}{k_{\mathrm{B}} T \sqrt{\overline{\mathcal{N}}}} .
$$


From above discussions, the time evolution of $A(q, t)$ and $g_{s}(q, t)$ at large $q$ deviates from the exponential decay at the beginning of the simulation.

For a large $q$, the time-evolution of $A(q, t)$ and $g_{s}(q, t)$ deviate from the exponential decay behavior. In the limit, when $1 \ll D R_{\mathrm{e} 0}^{2} t \mathbf{q}^{4} \ll\left(\mathbf{q} R_{\mathrm{e} 0}\right)^{4}$, the single-chain dynamic structure factor scales like $g_{s}(\mathbf{q}, t) \sim \exp \left(-2 \sqrt{D R_{\mathrm{e} 0}^{2} t \mathbf{q}^{4}} / 12 \pi\right)$ [64].

This result can also be quantitatively obtained by the assumption that the displacements of different subunits of the polymer chain can be conceived as motions of uncorrelated blobs of segments with a blob size that increases from the segment scale to the chain scale within the Rouse time, leading to a subdiffusive behavior on short time scales [120]. The time-dependent behavior of $A(q, t)$ at a large $q$ suggests that the Onsager coefficient is not only nonlocal in space, it is also nonlocal in time.

\subsubsection{Conclusions}

In this section, we provide a numerical scheme to directly measure the Onsager coefficient in the particle-based simulations. Although we only focus on the linear polymer chains with a simple Rouse dynamics, this method is rather general and can be easily extended to measure the Onsager coefficient in various polymer architectures and dynamics.

Based on the collective dynamic equation in Eq. 3.74, we can preliminary estimate the Onsager coefficient by the RPA. We find evidence that the Onsager coefficient is time-dependent. In the high-frequency regime with a small wavelength, the displacement of different segments are subdiffusive. 


\subsection{Minimum free-energy path}

\subsubsection{Illustration of string method}

The free-energy landscape is a functional of a collective variable, $m$ (e.g., the composition of two species in homopolymer blends). It captures important information for the structure formation process. Because it not only characterized the most probable transition path but also provides a one-dimensional reaction coordinate for the process. To understand the kinetics of block copolymers, it is computationally impossible to explore the full free-energy landscape in block copolymers. Because the dimension of a collective variable is very large.

For a given process, it is possible to understand its mechanism by exploring part of the free-energy landscape, i.e., computing the minimum free-energy path (MFEP) $[39,125]$. A MFEP that connects two ordered phases is computed which gives the information of the shape, size, and free-energy barrier of critical nuclei. Most importantly, the MFEP is used to investigate defect annihilation processes [126]. The MFEP also provides important insights for biological processes, e.g., the fusion and fission process in membranes.

The MFEP is defined by a series of order parameters, $m_{\alpha}(\vec{r})$ which is indexed by a continuous contour variables $\alpha$, where $0 \leq \alpha \leq 1$. These states $\left\{m_{\alpha}\right\}$ satisfy the condition that the gradient of the chemical potential, $\mu$ on the free-energy landscape perpendicular to the path vanishes, i.e., $\mu_{\perp}=0$, for all the states $\alpha$ with

$$
\begin{aligned}
\mu_{\perp}\left[m_{\alpha}\right](\vec{r}) \equiv & \mu\left[m_{\alpha}\right](\vec{r}) \\
& -\frac{\mathrm{d} m_{\alpha}(\vec{r})}{\mathrm{d} \alpha} \frac{\int \mathrm{d} \vec{r}\left[\mu\left[m_{\alpha}\right](\vec{r}) \frac{\mathrm{d} m_{\alpha}(\vec{r})}{\mathrm{d} \alpha}\right]}{\int \mathrm{d} \vec{r}\left(\frac{\mathrm{d} m_{\alpha}(\vec{r})}{\mathrm{d} \alpha}\right)^{2}}
\end{aligned}
$$

We employ the string method to find the MFEP ([123]), which consists of a two-step cycle procedure: (i) Minimizing the free energy $\mathcal{F}$ by evolving the morphologies according to the chemical potential $\mu$,

$$
m_{\alpha}(\vec{r}, t+\Delta t)=m_{\alpha}(\vec{r}, t)-\Delta t \mu\left[m_{\alpha}\right](\vec{r})
$$


(ii) New morphologies $m_{\alpha}(\vec{r}, t+\Delta t)$ are reparameterized by the cubic spline interpolation along $\alpha$ for the fixed position $\vec{r}$ to restore uniform distance of $\Delta \alpha$ between adjacent states. The distance $\Delta \alpha$ between two adjacent states $m_{\alpha_{1}}(\vec{r})$ and $m_{\alpha_{2}}(\vec{r})$ is defined as,

$$
\Delta \alpha \propto \int \mathrm{d} \vec{r}\left[m_{\alpha_{1}}(\vec{r})-m_{\alpha_{2}}(\vec{r})\right]^{2}
$$

Starting and ending states $\alpha=0$ and $\alpha=1$ are only subjected to the chemical potential $\mu$, they will not be extrapolated by the cubic spline interpolation. From the definition of the MFEP, it is possible that there might exist many MFEPs in the complex free-energy landscape. So it is crucial to provide proper initial morphologies for the string calculation. To study the structure formation process, we can use the initial morphologies, which are obtained by observing the time evolution in particle-based simulations.

To calculate a MFEP, we also need to determine an appropriate numerical method to compute the chemical potential, $\mu$. In previous sections, we have proposed several numerical strategies. For diblock copolymers, the external potential method (EPD) uses the collective variable $W$ which is conjugated to the local density composition variable $m$ in SCFT as the order parameter. This simple strategy is applied to investigate the nucleation of various ordered phases in block copolymers [127]. In this thesis, we use the umbrella chemical potential in conjunction with the SCFT method to derive the chemical potential for block copolymers. There are two advantages of this strategy: (i) We can directly use the composition of the two species as the order parameter. (ii) The umbrella potential method is not limited to a specific system. It can be easily migrated to a more complex system.

To evolve the order parameter, $m$, we use the simple dynamics of model A (i.e., the Allen-Cahn dynamics [128]) in Eq. 3.76. This scheme is computationally simple with a high efficiency. We can also employ the Cahn-Hilliard dynamics in Eq. 3.42 to evolve the order parameter that characterize the diffusive dynamics of the corresponding particle system.

It is also possible to use the field-theoretical umbrella sampling method in conjunction with the SCMF algorithm to calculate the MFEP. This numerical scheme 

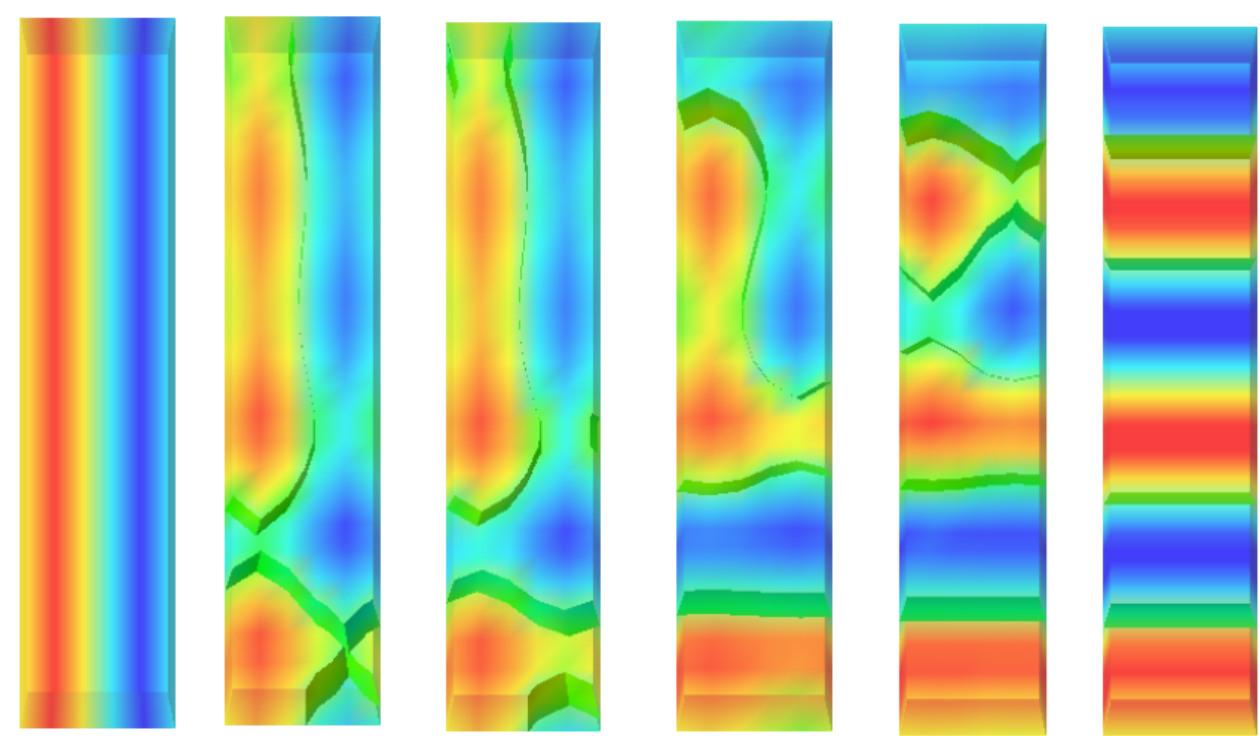

$$
\alpha=0
$$

$\alpha=0.25 \alpha=0.3$

$\alpha=0.5$

$\alpha=0.65$

$\alpha=1.0$

FIGURE 3.8: We present the snapshots of the MFEP that connects the metastable, layered lamellae to the stable, standing lamellae.

avoids cumbersome derivations to obtain the corresponding SCFT equations. From our discussions in the previous section, in the limit of $\overline{\mathcal{N}} \rightarrow \infty$, the SCFT and the particle-based simulations are equivalent. The deficiency is that in the particle-based simulations, the string method cannot compute the free-energy barrier with a high precision due to the thermal fluctuations.

In the following, we will use the string method to investigate collective phenomena in diblock copolymers and triblock copolymers, respectively.

\subsubsection{Orientation transition of lamellar-forming diblock copoly- mers}

In this section, we investigate a transition process from a metastable, layered lamella to the equilibrium, standing lamellar state in diblock copolymer melts. The size of system is $4 \times 1 \times 1 R_{\mathrm{e} 0}^{3}$ with a periodic boundary condition. The incompatible parameter $\chi N=16$. 


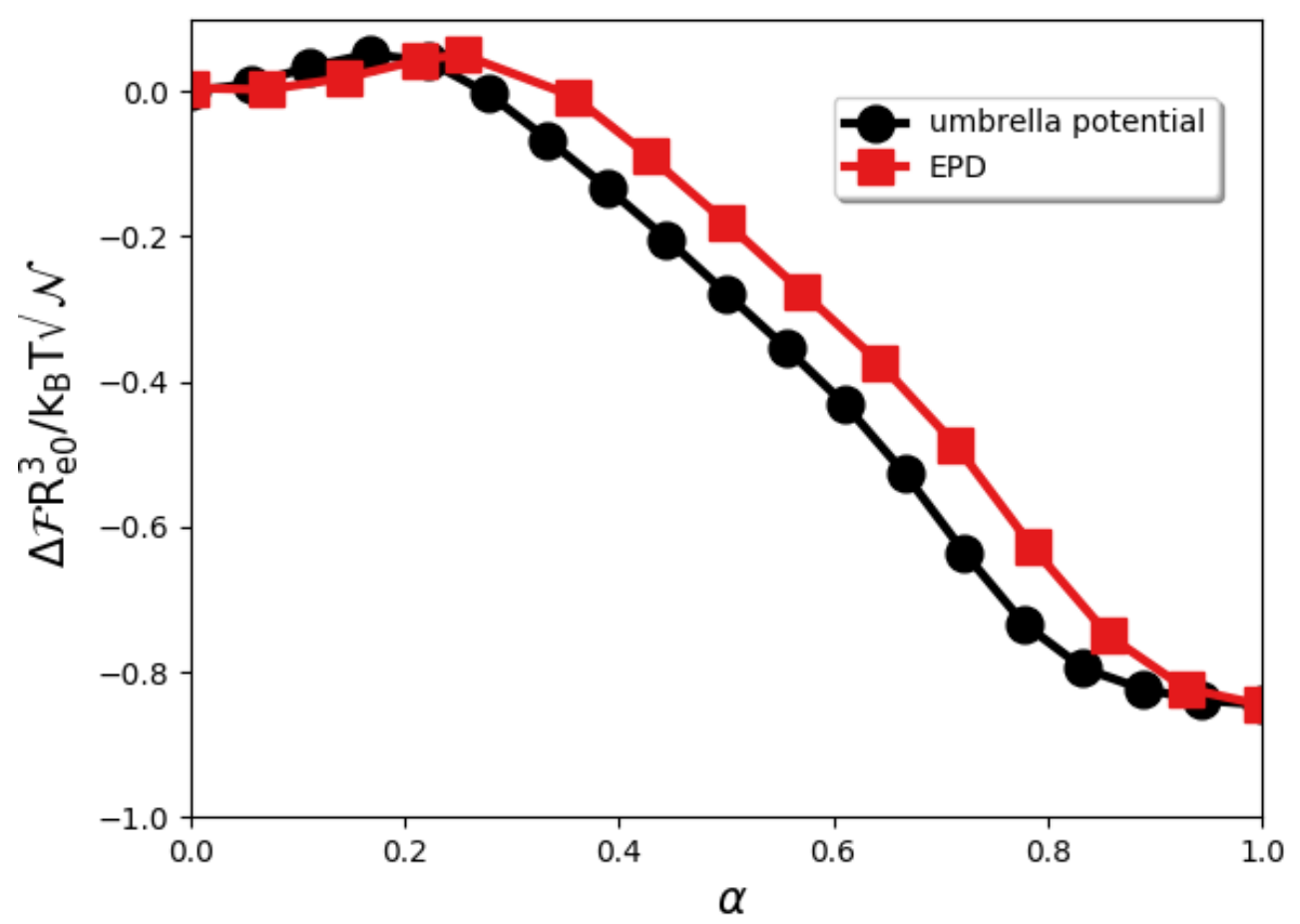

FIGURE 3.9: The free-energy difference $\mathcal{F}\left[m_{\alpha}\right]$ is plotted as a function of reaction coordinates $\alpha$. The snapshots are shown in Fig. 3.8.

We use an umbrella potential to obtain the initial, metastable, layered state in the particle-based simulation. After we remove the umbrella potential, the system evolves to the equilibrium state (i.e., the standing lamellar state). The initial states for the MFEP calculation is obtained from the particle-based simulation.

We use the string method combined with the umbrella potential method to derive the MFEP and the discretization of the string is 18 . The points on the string are redistributed by the cubic spline interpolation. The starting point of the string, $\alpha=0$ and the ending point, $\alpha=1$ are not fixed. These two points are meta-stable and stable, respectively. In the end, the string converges to the MFEP. We present the snapshots in the Fig. 3.8.

The free-energy difference, $\Delta \mathcal{F}$ between the state $m_{\alpha=0}$ and other states on the string as a function of the reaction coordinates, $\alpha$ is plotted on the Fig. 3.9. We also compute the MFEP via the EPD scheme. The only difference between these two approaches is that the order parameter in EPD is the auxiliary fields. As a result, the distance between the two neighboring points on the MFEP from the EPD is different from the umbrella potential method. However, the quantities 
that we are interested in (e.g., the excess free energy and the free-energy barrier) are quantitatively the same.

We are particularly interested in determining the free-energy barrier $\Delta F_{\mathrm{b}} / k_{\mathrm{B}} T$ of the transition. It determines the inverse rate of the transition between the two thermal states. According to Kramers' theory [129], the corresponding Boltzmann factor $\exp \left(-\Delta F_{\mathrm{b}} / k_{\mathrm{B}} T\right)$ dictates the transition rate.

The saddle point is at approximately $\alpha \approx 0.2$ and the barrier is $\Delta \mathcal{F} / k_{\mathrm{B}} T \sqrt{\overline{\mathcal{N}}} \approx$ 0.05128. In an experiment, this invariant degree of polymerization, $\overline{\mathcal{N}}=128^{2}$, which gives a small free-energy barrier, $\Delta \mathcal{F} / k_{\mathrm{B}} T \approx 6.258$. This small freeenergy barrier can be easily overcome by the thermal fluctuations.

\subsubsection{Impact of the molecular architecture on the structure for- mation process}

In a diblock copolymer melt, the influence of the temperature on the structure formation process is intensively studied by the string method [126, 130]. For example, the free-energy barrier of the annihilation of the B-core dislocation dipole increases linearly with the Flory-Huggins parameter, $\chi N$ [130].

However, the influence of the molecular architecture on the structure formation process is not clear. For lamellar-forming block copolymers, we can easily find many similar defects in the structure formation process. In this section, we will only focus on a defect removal process (i.e., the B-core dislocation dipole) in triblock copolymers and diblock copolymers, respectively.

The triblock copolymers are obtained by replacing half of the B block with the $\mathrm{C}$ block in symmetric diblock copolymers (i.e., $f_{A}=0.5, f_{B}=0.25$, and $f_{C}=0.25$ ). The scheme representation of the triblock copolymers is shown in Fig. 3.11. By tuning the incompatible parameters, $\chi_{B C} N$ between B blocks and C blocks, we can find a smooth transition from the symmetric diblock copolymer to the $\mathrm{ABC}$ triblock copolymer. The incompatible parameter between A block and $\mathrm{B}(\mathrm{C})$ block is $\chi_{A B} N=30\left(\chi_{A C} N=30\right)$. We implement the calculation in a volume of $8 \times 8 \times 1 R_{\mathrm{e} 0}^{3}$ with periodic boundary conditions. 


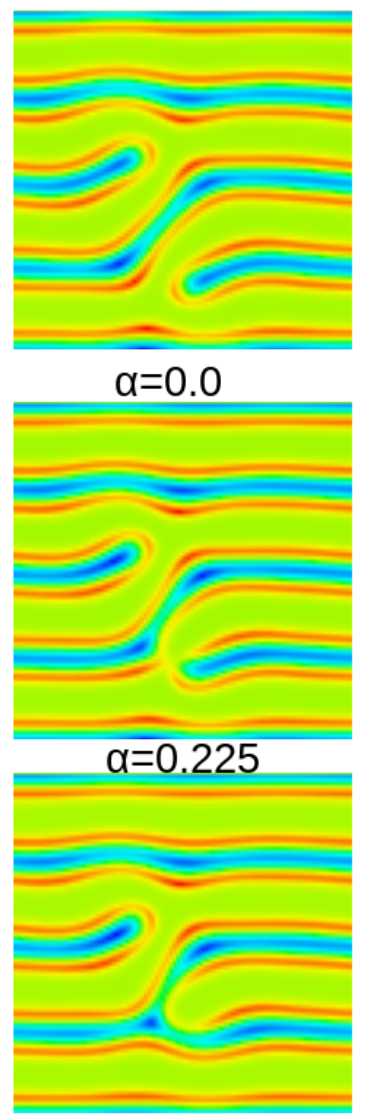

$\alpha=0.5$

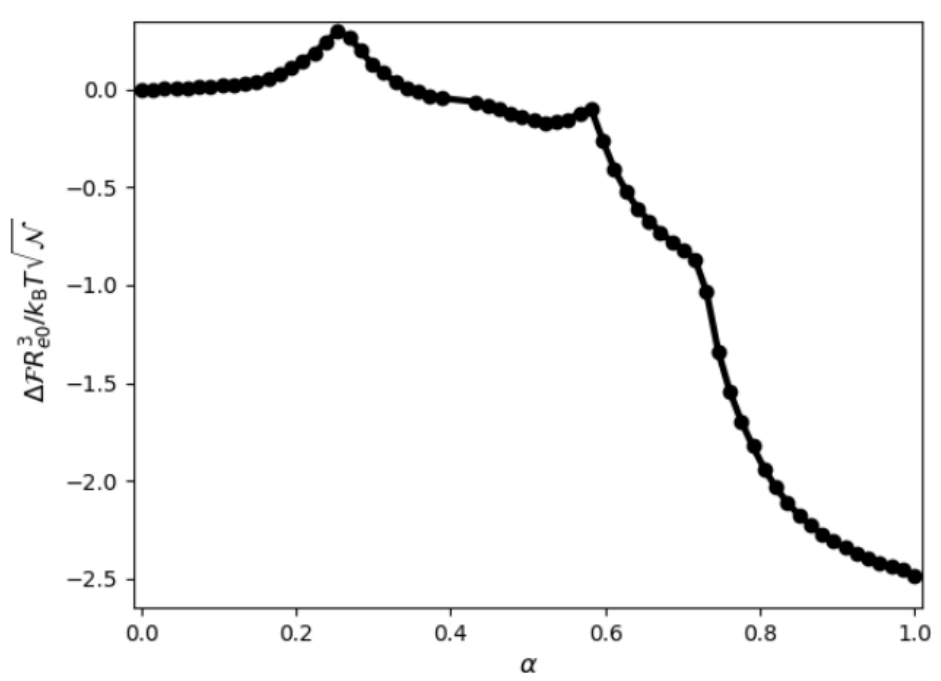

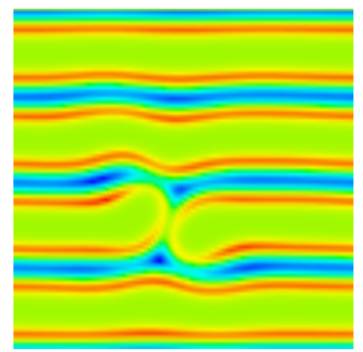

$\alpha=0.725$

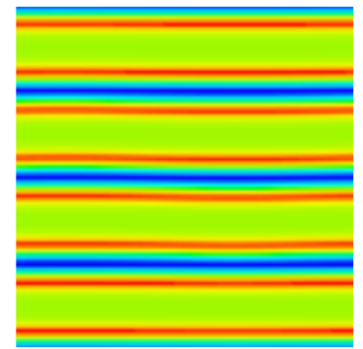

$\alpha=1.0$

FIGURE 3.10: The MFEP snapshots of the removal process of the B-core dislocation dipole in diblock copolymers. The order parameter of the snapshots is $\phi_{B}-\phi_{C}$. The free-energy difference $\mathcal{F}\left[m_{\alpha}\right]$ of the MFEP is plotted as a function of reaction coordinates $\alpha$.

The defect annihilation process of the B-core dislocation dipole is well studied [130]. As shown in the Fig. 3.9, the removal process in symmetric block copolymer melts (i.e., $\chi_{B C} N=0$ ) is triggered by breaking one connection between two A domains. This process gives the largest free-energy barrier which determines the defect-removal rate. Subsequently, the morphology relaxes to a meta-stable state with a small free-energy barrier to overcome. This metastable state is eliminated by breaking the remaining A connection. In the end, the two disconnected A lamellar domains are connected without a barrier.

Similarly, we obtain the MFEP for triblock copolymers to eliminate this defect. We use the chemical potential method in conjunction with SCFT to compute the chemical potential with three components $A, B$ and $C$. We consider only the incompressible system with $\Phi_{C}=1-\Phi_{A}-\Phi_{B}$. We use the densities $\Phi_{A}$ and $\Phi_{B}$ 


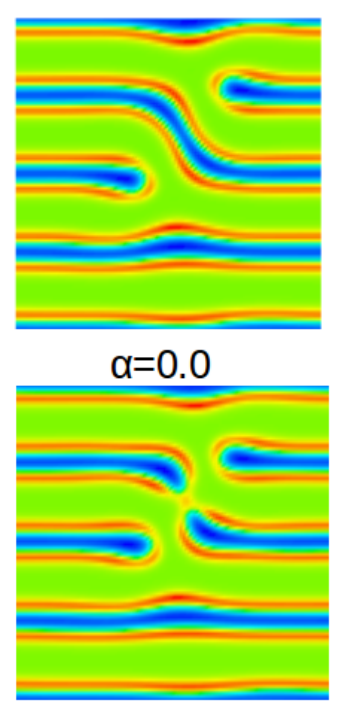

$\alpha=0.3$

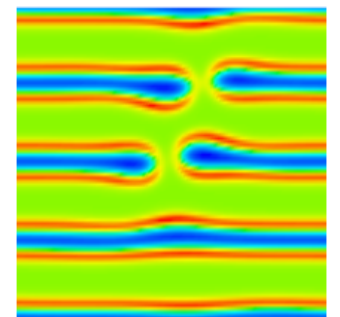

$\alpha=0.5$

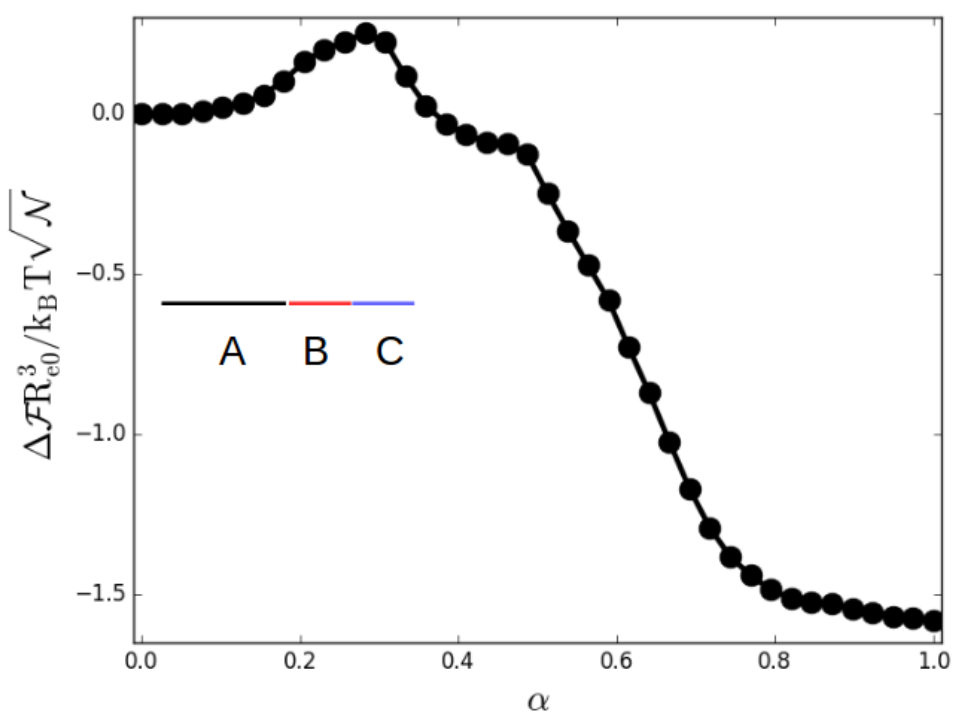

FIGURE 3.11: The MFEP snapshots of the removal process of the B-core dislocation dipole in triblock copolymers. The order parameter of the snapshots is $\Phi_{B}-\Phi_{C}$. The free-energy difference $\mathcal{F}\left[m_{\alpha}\right]$ of the MFEP is plotted as a function of reaction coordinates $\alpha$.

as the order parameter. The free-energy functional is denoted as, $\mathcal{F}_{\text {tri }}\left[\Phi_{A}, \Phi_{B}, 1-\right.$ $\left.\Phi_{A}-\Phi_{B}\right]$.

The dynamic evolution equation of the densities with Model A dynamics is obtained as,

$$
\begin{aligned}
& \Phi_{A}^{t+\Delta t}=\Phi_{A}^{t}+\Delta t \mu_{A}\left[\Phi_{A}, \Phi_{B}, \Phi_{C}\right] \\
& \Phi_{B}^{t+\Delta t}=\Phi_{B}^{t}+\Delta t \mu_{B}\left[\Phi_{A}, \Phi_{B}, \Phi_{C}\right]
\end{aligned}
$$


where $\mu_{A}$ and $\mu_{B}$ are chemical potentials defined as,

$$
\begin{aligned}
& \mu_{A}=\frac{\delta \mathcal{F}_{\text {tri }}}{\delta \Phi_{A}} \\
& \mu_{B}=\frac{\delta \mathcal{F}_{\text {tri }}}{\delta \Phi_{B}}
\end{aligned}
$$

The initial states $m_{\alpha}(t=0)$ along the string is obtained by uniformly discretizing the two starting and ending states. Similar to the standard string method, we can obtain a converged path in Fig. 3.10.

The resulting MFEP in triblock copolymers differs a lot from the defect annihilation process in diblock copolymers. As shown in Fig. 3.11, the defect annihilation process is triggered by a connection between the two dislocations in triblock copolymers. Meanwhile, we can find that there is only one free-energy barrier on the MFEP of the triblock copolymers. As a comparison, there are two free-energy barriers on the MFEP for the diblock copolymers.

The reason for this difference is that the meta-stable morphology $\alpha=0.5$ in diblock copolymers is unstable in triblock copolymers. As a result, the MFEP in diblock copolymers will converge to the MFEP in triblock copolymers. As a contrast, the MFEP in triblock copolymers is also a MFEP in diblock copolymers. However, the free-energy barrier of this process is approximately $0.0118 k_{\mathrm{B}} T$ per chain, which is much larger than the original MFEP in diblock copolymers which is only $0.0046 k_{\mathrm{B}} T$ per chain.

Above discussions suggest that the molecular architecture has an impact on the defect annihilation process. It alters both the defect annihilation process and the free-energy barriers. As a result, by increasing $\chi_{B C} N$, there is a transition point that separates the two defect annihilation process. 


\section{Chapter 4}

\section{Pattern formation in diblock copolymer melts}

\subsection{Qualitative behavior of diblock copolymers in a thin film}

In this section, we will use computer simulations (i.e., the SCMF, particle-based simulation) to study the kinetics of structure formation for symmetric diblock copolymer melts in a quasi-two-dimensional system. The thickness of the system is determined as $1 R_{\mathrm{e} 0}$, which is smaller than the typical lamellar spacing. As a result, the resulting structure of the system is two dimensional, which resembles a thin film. The system is characterized by a large extension $L$ in the two lateral directions. The square shape geometry of the system facilitates the detection of the defects and allows us to address large lateral system sizes.

The system is initialized to mimic the high-temperature state, $\chi N=0$ by randomly distributing Gaussian polymer chains. At time $t=0$, the incompatible parameter, $\chi N$ between unlike segments is switched to a value which is above the order-disorder transition (ODT). This quenching process mimics e.g., rapid solvent evaporation.

The ODT of symmetric diblock copolymer is at $\chi N \approx 10.495 \ldots[17,19]$, obtained by the RPA or the SCFT calculation. In simulations or experiments, composition fluctuations shift the ODT point to $\chi N \approx 10.495+41.022 \overline{\mathcal{N}}^{-1 / 3}$ and 
(a)
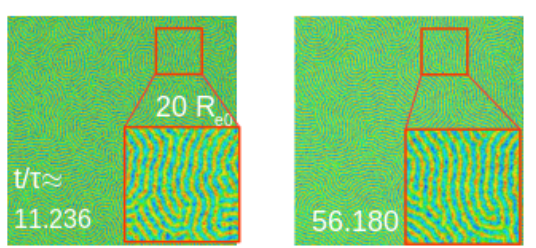

$\chi \mathrm{N}=15, \mathrm{Nbar}=128^{\wedge} 2$

11.236

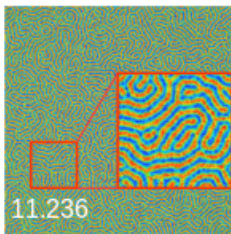

$100 \mathrm{R}_{\mathrm{e} 0}$
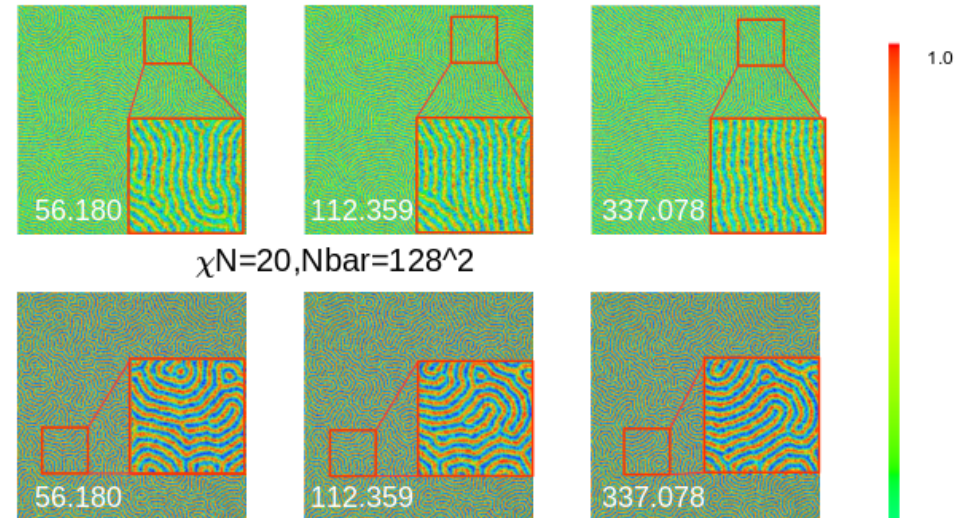

$\chi \mathrm{N}=20, \mathrm{Nbar}=128^{\wedge} 2$
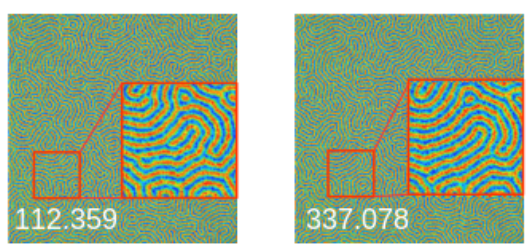

(b) $t / 1 \approx 561.797$ $X \mathrm{~N}=15$

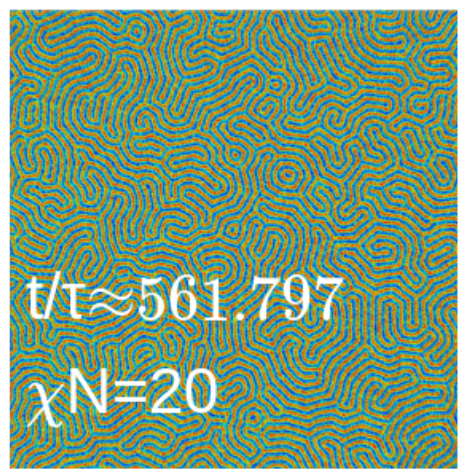

(c)
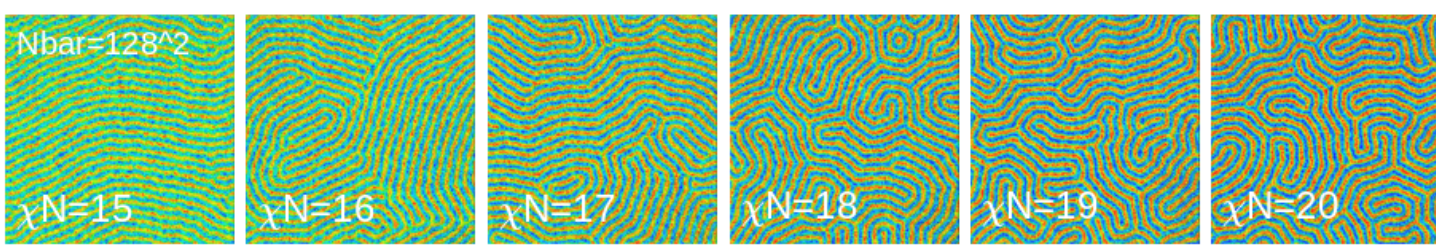

$40 R_{\mathrm{e} 0}$

FIGURE 4.1: (a) Snapshots of the time evolution of two SCMF, particle-based simulations in a thin film system at $\chi N=15$ and $\chi N=20$, respectively. The lateral system extension, $L$ is $L=100 R_{\mathrm{e} 0}$ and the thickness of the thin film $1 R_{\mathrm{e} 0}$. The insets show enlarged portions of the morphology in a small region of $20 \times 20 R_{\mathrm{e} 0}^{2}$ whose position is indicated by the red square in the main panels. Morphologies are obtained at times $t / \tau \approx 11,56,112$ and 337 after the quench from the disordered phase are presented. (b) Enlarged snapshots of morphologies at time $t \approx 562 \tau$ after the quench to $\chi N=15$ and $\chi N=20$, respectively. (c) Snapshots at approximately $337 \tau$ by particle-based simulations after the quench for six different values of $\chi N=15,16,17,18,19$ and 20 . The system size is $L=40 R_{\mathrm{e} 0}$. 
alter the character of the transition from second order to first order [68]. For a typical value of $\overline{\mathcal{N}}=128^{2}$ in an experiment, the system exhibits lamellar phase for $\chi N=15$.

Due to phonons and dislocation defects, it is difficult for diblock copolymer thin films to exhibit truly long-range orders. The free-energy scale in polymeric systems is set by $k_{\mathrm{B}} T \sqrt{\mathcal{N}} D / R_{\mathrm{e} 0}$, where $D$ denotes the thickness of the quasi-onedimensional system. For example, the excess free-energy of an isolated dislocation pair at $\chi N=21$ (below which the metastable dislocation pair is unstable) is approximately $F_{\mathrm{d}} \approx 1.04 k_{\mathrm{B}} T \sqrt{\overline{\mathcal{N}}} D / R_{\mathrm{e} 0} \approx 133 k_{\mathrm{B}} T$ [130]. As a result, the equilibrium density of this kind of defect is vanishingly small (i.e., the equilibrium distance between dislocation pairs is on the order $\left.L_{\mathrm{d}} \sim R_{\mathrm{e} 0} \exp \left(\Delta F_{\mathrm{d}} / 2 k_{\mathrm{B}} T\right) \gg L\right)$. It is also found that the compression modulus of the lamellar structure scales like $B=k_{\mathrm{B}} T \sqrt{\overline{\mathcal{N}}} \frac{D}{R_{e 0}^{3}} f_{B}(\chi N)$, where $f_{B}$ increases with incompatibility [131]. As a result, on the length scale that we studied in particle-based simulations, an equilibrium structure corresponds to a defect-free lamellar structure. Although the free-energy cost of defects and deformations increases with incompatibility, $\chi N$, we observe that the order of a pattern obtained by a quench from the disordered phase, at a given, fixed time is less developed at large $\chi N$. Therefore our conclusion is that the deviations from a defect-free lamellar structure observed in our particle-based simulations cannot be explained by equilibrium fluctuations around the perfect equilibrium state, instead, stem from the kinetics of structure formation.

We first qualitatively examine the time evolution of two independent particlebased simulations at $\chi N=15$ and 20, respectively. Systematically quantifying and investigating the structure formation process in symmetric diblock copolymer melts are presented in the following sections. In Fig. 4.1 (a), timedependent morphologies of particle-based simulations at $\chi N=15$ and $\chi N=$ 20 are presented for the lateral size, $L=100 R_{\mathrm{e} 0}$. Time in these simulations is measured in units $\tau$, which is defined as $\tau=R_{\mathrm{e} 0}^{2} / D$, where $D$ is the selfdiffusion coefficient of a polymer chain in the disordered state.

Diblock copolymers are weakly segregated at $\chi N=15$. At $t \approx 11.236 \tau$, we find that the morphology consists of many lamellar grains. The lamellar order is established within these lamellar grains whose orientation and positional registration differs. Domain connections across a grain boundary will break and reconnected within one $\tau$. As a result, domain connections at grain boundary 
are transient and this allows for the movement of grain boundaries, which results in a gradual coarsening of the grain structure. At the end of our simulation time, $t \approx 562 \tau$ cf. Fig. 4.1 (b), grains with orientational order can increase up to nearly half of the system size.

At intermediate segregation regime, $\chi N=20$, polymers are segregated more strongly. The composition contrast between unlike species domains is much larger but the grain-coarsening speed slows down dramatically compared to the structure ordering process at $\chi N=15$. In the snapshots of our particlebased simulations, we observe a fingerprint-like morphology at $t \approx 11 \tau$ where only local order is established. Similar to a micellar solution, there is a characteristic length scale (i.e., the domain spacing) but no grain structures with a scale separation between an extended lamellar ordered interior of a grain and narrow grain boundaries, can be detected. The morphology is almost "frozen" in our particle-based simulations, i.e., the topology of the domain structures remains almost unaltered over several hundred $\tau$, as shown in the insets of Fig. 4.1 (a). It is very protracted to establish long-range order and outside the times that our large-scale, particle-based simulation can access.

From above two examples, it is quantitatively demonstrated that the ordering time dramatically increases from $\chi N=15$ to $\chi N=20$. To further illustrate this behavior, we show the snapshots of smaller systems, $L=40 R_{\mathrm{e} 0}$, at $t \approx 337$ from $\chi N=15$ to 20. In Fig. 4.1 (c), at a fixed time after a quench from the disordered state, we observe that the order gradually decreases as a function of incompatibility, $\chi N$. At $\chi N=15$, we find that the system of lateral size $L=40 R_{\mathrm{e} 0}$ consists of a single lamellar grain with some undulations and a few dislocation defects. For a larger $\chi N=16$, we find that there are multiple grains on the morphology with a visible grain boundary. The size of grains shrinks and the defect density increases, upon increasing the incompatible parameter, $\chi N$. The morphology cannot be conceived as grain structures with a clear separation between an ordered interior of a grain and grain boundaries.

\subsection{Commensurability and lamellar spacing}

As shown in Fig. 4.1, although the morphologies obtained by the particle-based simulations are absent of long-range order, the characteristic domain size is well 


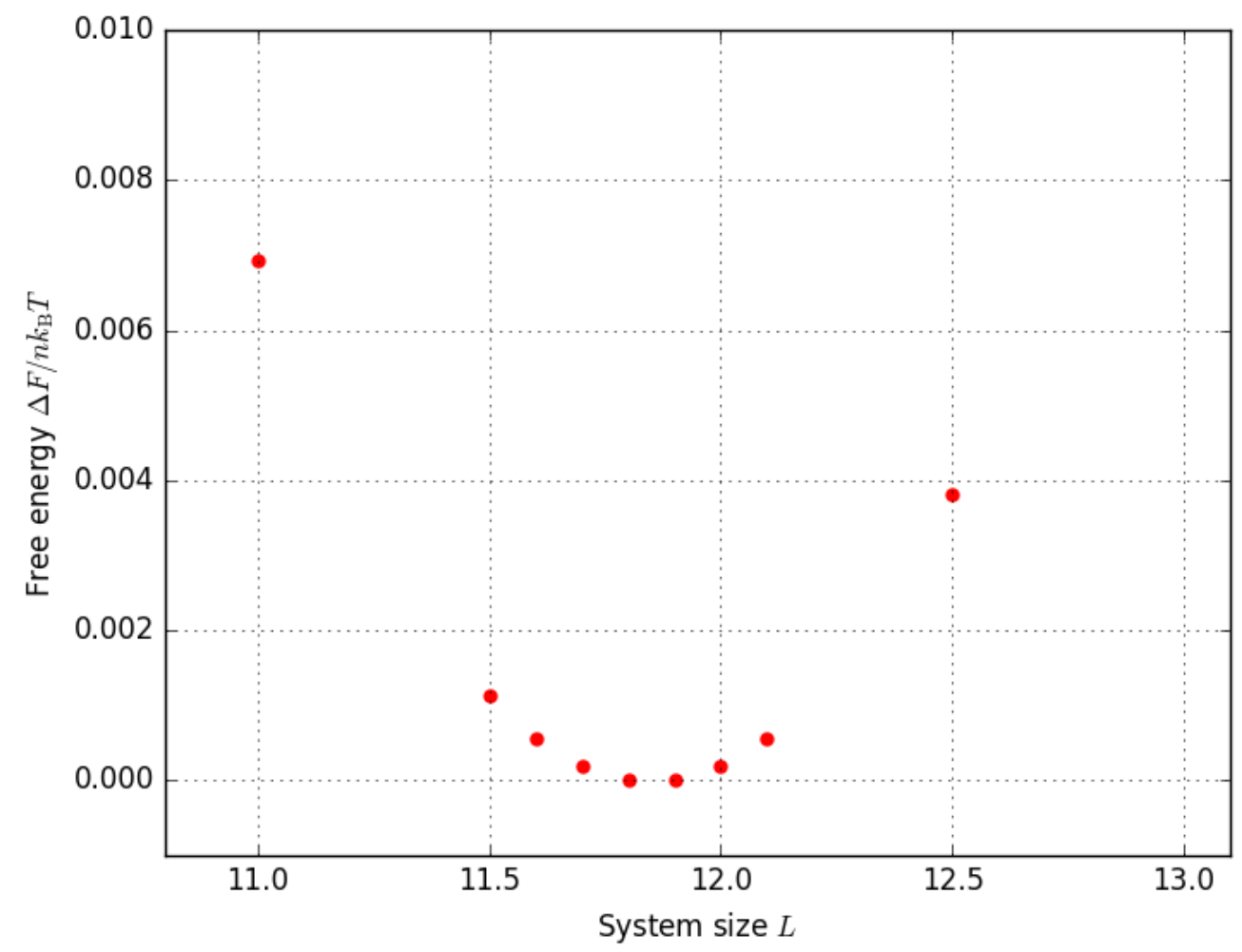

FIGURE 4.2: The free-energy difference of a single polymer chain between morphologies with various lamellar spacing and the morphology with the optimal lamellar spacing obtained by the SCFT.

established (as defined by the location of the peak of the collective structure factor). It does not change in time and agrees to the lamellar spacing of the equilibrium structure.

The lamellar spacing is determined by the Flory-Huggins parameter, $\chi N$ and can be measured by calculating the structure factor or by comparing the free energies.

The morphology is characterized by the structure factor of composition fluctuations

$$
S(\vec{q}, t) \equiv \frac{1}{V}\left\langle\left|\int \mathrm{d} \vec{r} e^{i \vec{q} \vec{r}} m(\vec{r}, t)\right|\right\rangle
$$

where $\langle\ldots\rangle$ denotes the time average. The lamellar spacing is estimated by the position of peak value,

$$
L_{\mathrm{s}}=2 \pi / q^{*}
$$

It gives the average value of the lamellar spacing for a morphology. 


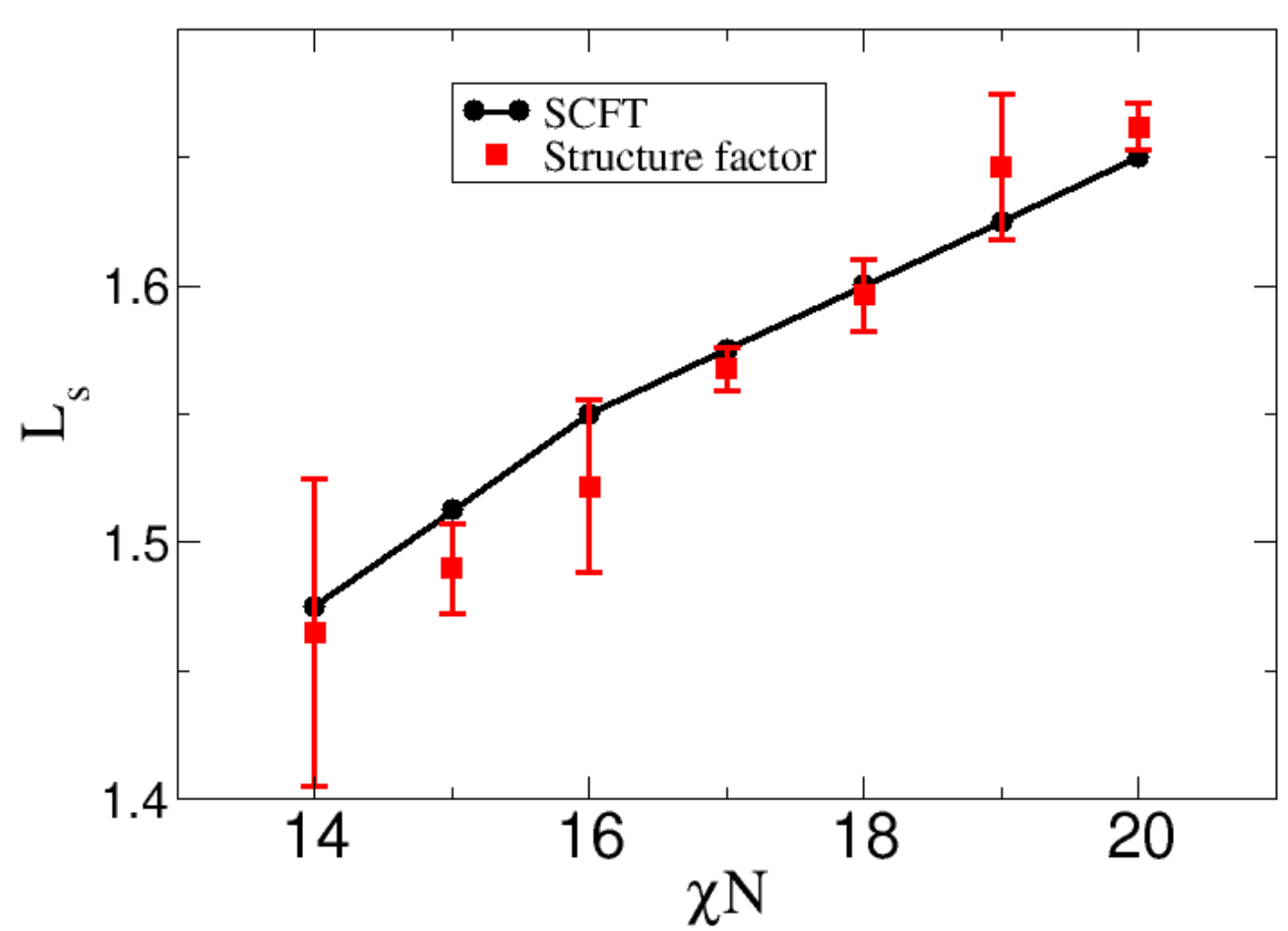

FIGURE 4.3: The optimal lamellar spacing as a function of the Florry-Huggins parameter, $\chi N$. Results obtained by the structure factor are averaged from 5 independent simulations after $10^{7} \mathrm{SMCs}$.

More precisely, the lamellar spacing at different $\chi N$ can be measured by comparing the free energies. We consider a morphology with a fixed number of lamellae (e.g., 8 lamellae) and identical orientations along the $x$-axis. By tuning the lateral extension, $L$, we can find an optimal system size $L^{*}$ which gives the lowest free energy per chain, i.e., the optimal system sizes which corresponds to the lamellar spacing.

For example at $\chi N=14$, we plot the free-energy difference between morphologies with different extension $L$ and the morphology which gives the smallest free energy. As shown in Fig. 4.2, there is only one minimal free energy which corresponds to the optimal system size $L^{*}=11.8 R_{\mathrm{e} 0}$, i.e., the lamellar spacing at $\chi N=14$ is $1.475 R_{\mathrm{e} 0}$.

As a comparison to the particle-based simulations, we use a value of $\overline{\mathcal{N}}=128^{2}$. After running the simulation for $10^{7} \mathrm{SMCs}$, we obtained a fingerprint-like morphology. The peak value, $q^{*}$ of the morphology ranges from $4.04224 R_{\mathrm{e} 0}^{-1}$ to 
$4.36946 R_{\mathrm{e} 0}^{-1}$ from 5 independent simulations. The resulting lamellar spacing ranges from $1.4379 R_{\mathrm{e} 0}$ to $1.5543 R_{\mathrm{e} 0}$, which is consistent with the SCFT calculation.

As shown in Fig. 4.3, we plot the lamellar spacing as a function of $\chi N$. We find that the lamellar spacing increases linearly with $\chi N$. The polymer chain is stretched by the repulsive force between unlike species and reach a balance between the entropy loss due to chain stretching and free-energy minimization by minimizing internal $\mathrm{AB}$ interfaces.

\subsection{Correlation length}

Intuitively, we try to quantify the structure formation process in diblock copolymer melts by measuring the structure factor, $S(|\vec{q}|)$ defined in Eq. 4.1. In Fig. 4.4 (a), we present the angularly averaged structure factor, $S(|\vec{q}|)$ at various $\chi N$. It has a peak at $\left|\vec{q}^{*}\right|=2 \pi / L^{*}$ that quantifies the lamellar spacing, $L_{\mathrm{s}}$. The position of the peaks is shifted to smaller wavevectors (i.e, the domain spacing increases), while increasing $\chi N$. The height of the peak value of $S\left(\overrightarrow{q^{*}}\right)$ is determined by two factors: the correlation length of the morphology and the degree of segregation that dictates the composition contrast of different domains.

The two-dimensional structure factor at $\chi N=15$ and 20 is presented in Fig. 4.4 (b). At $\chi N=20$, this snapshot of the structure factor is isotropic, i.e, there are many domains with different orientations in the system. For $\chi N=15$, the structure factor exhibits a strong angular variation, which indicates the presence of large grains (i.e., a size on the order $L$ ).

Although the structure factor contains the information of the size of lamellar grains, it is not easy to directly extract this information from the structure factor. To obtain the correlation length from the morphology, we measure the correlation function, $g_{r}$ as an alternative, which corresponds to the structure factor $S(q)$, in real space,

$$
g_{r}(\Delta r)=\frac{1}{2 \pi r \Delta r L^{2}} \int_{L^{2}} \mathrm{~d}^{2} \vec{r} \int_{\left|\vec{r}-\vec{r}^{\prime}\right|=\Delta r} \underset{\mathrm{d}^{2}}{\overrightarrow{r^{\prime}}} \bar{m}(\vec{r}) \bar{m}\left(\overrightarrow{r^{\prime}}\right) .
$$



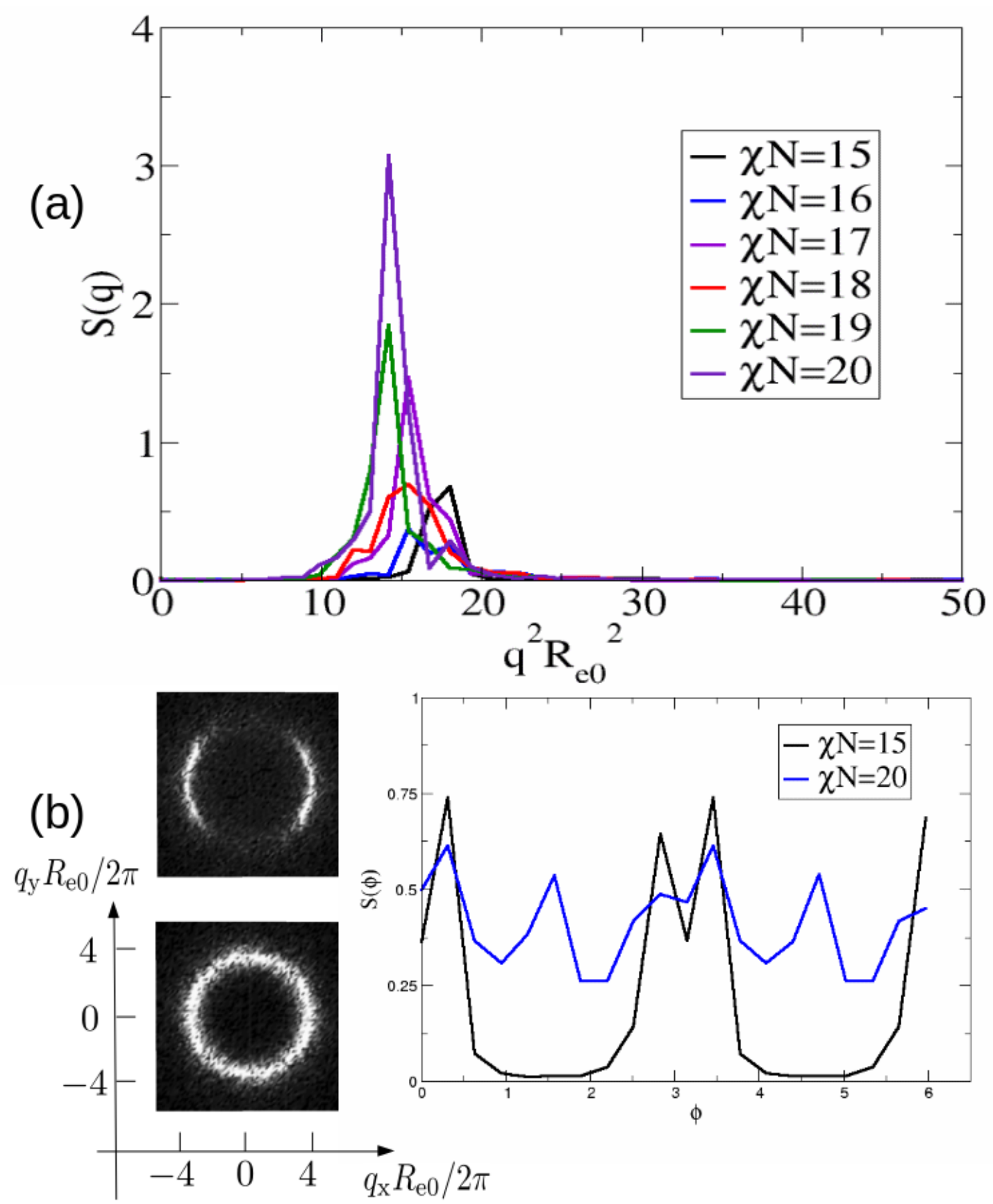

FIGURE 4.4: (a) $S(\vec{q})$ (i.e., structure factor of composition fluctuations ) for various incompatibilities in the symmetric diblock copolymer melt. (b) $S(\vec{q})$ on the $x y$ plane and the corresponding angular dependence from the instantaneous morphologies shown in Fig. 4.1 (c). 


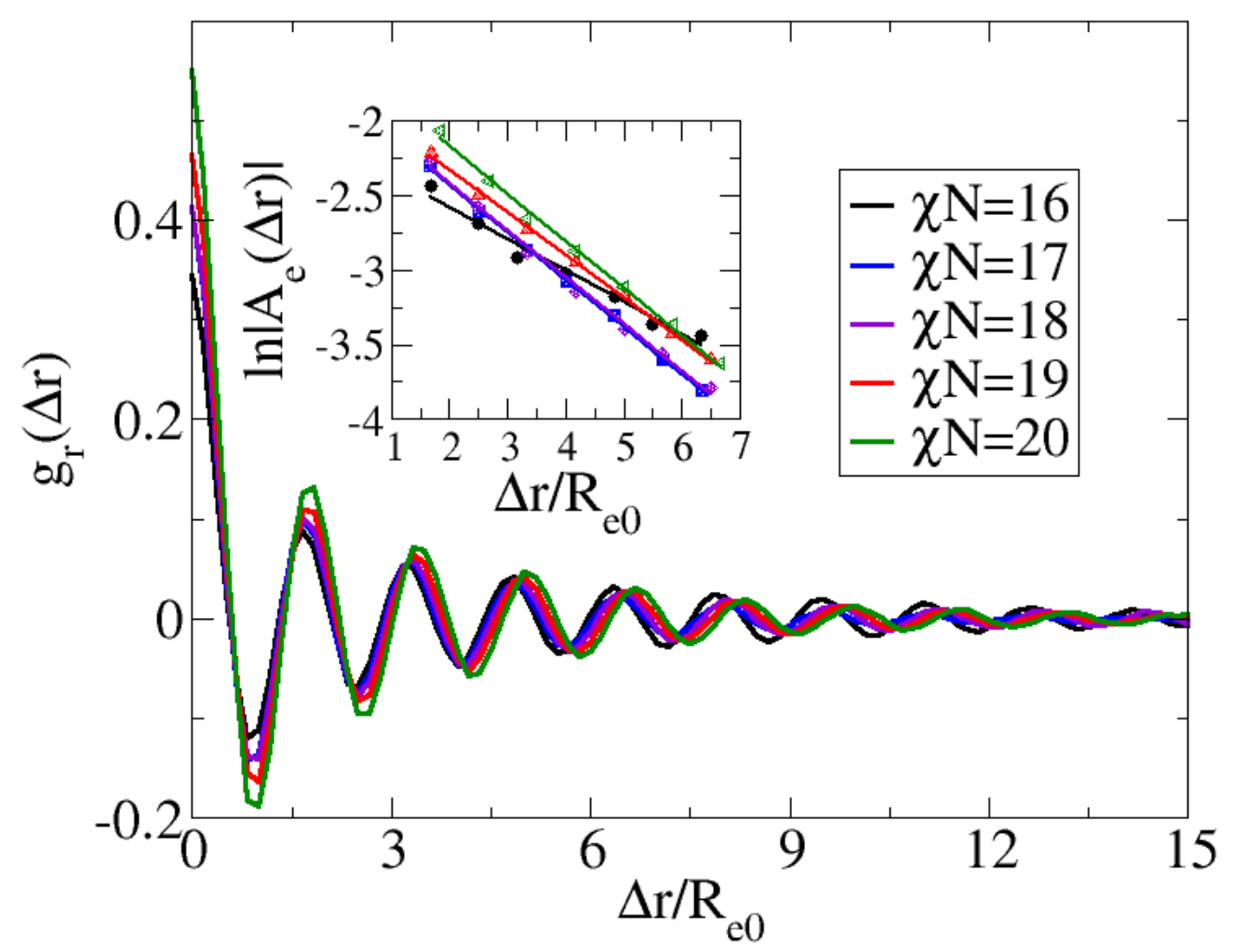

FIGURE 4.5: The correlation function, $g_{r}$ for six independent simulations with different $\chi N$ is obtained as a function $\Delta r\left(R_{\mathrm{e} 0}\right)$. The morphologies of these simulations are shown in Fig. 4.1 (c). Inset: The logarithm of the absolute value of extreme values of $\left.g_{r}, \ln \mid A_{e}(n, t, \Delta r)\right) \mid$ as a function of distance $\Delta r$.

On the two-dimensional morphology, $\Delta r \equiv|\vec{r}-\vec{r}|$ is the distance between two points and the morphology is averaged over $z$-axis denoted by $\bar{m}(x, y)$ as the $z$-averaged order parameter.

In Fig. 4.5, we plot $g_{r}(\Delta)$ as a function of $\Delta r$ from $\chi N=16$ to $\chi N=20$ for the morphologies shown in Fig. 4.1 (c). We find that $g_{r}(\Delta r)$ shows an oscillation with a period $L_{\mathrm{s}}$ and exponential decay that characterizes the correlation length, $\xi$, of the order. As expected, the lamellar spacing increases with $\chi N$ from $\chi N=$ 16 to $\chi N=20$. The value of the correlation function at short distances, $\Delta r$ also increases with $\chi N$ because it reflects the segregation between unlike segment species. From the simulations data, the correlation function is described with the form,

$$
g_{r}(\Delta r) \sim e^{-\Delta r / \xi} \cos \left(\frac{2 \pi \Delta r}{L_{\mathrm{s}}}\right) .
$$

The size of lamellar grains is characterized the correlation length, $\xi$. 
In order to obtain the correlation length, $\xi$, we use the extreme points of the correlation function, $g_{r}(\Delta r)$. We use $A_{j}=g_{r}\left(\Delta r_{j}\right)$ and $\Delta r_{j}$ to denote the absolute value of the $j^{\text {th }}$ extreme point of $g_{r}$. $\ln A_{j} v s . \Delta r_{j}$ shows a linear behavior in the inset of Fig. 4.5. This linear behavior dictates the exponential decay and we can easily read off the correlation length, $\xi$ from the inverse slope.

To analyze the growing of correlation length at different $\chi N$, we average the results over multiple independent ordering processes that are obtained by the particle-based simulations. In order to avoid the finite-size effect (i.e., the value of the correlation length depends on the system size), we compare the time dependence of the correlation length at $\chi N=16$ and 20 for different system sizes in Fig. 4.6. Each point of $\xi$ is averaged over five independent simulations.

We find that there is a pronounced slowing down of the growth of the correlation length. On the double-logarithmic plot, there are two slopes of the correlation length. The correlation length, $\xi$ becomes smaller at the final slope of $\xi(t)$. We concluded that this is not a finite-size effect because of two reasons: (i) the results for different system size exhibit similar results within the statistical errors. (ii) we can observe correlation lengths for the same system size at smaller $\chi N=16$. Qualitatively, we can observe the absence of the finite-size effect by comparing the snapshots in Fig. 4.1.

The correlation length at a small incompatible parameter, $\chi N=16$ is larger and continues to grow at later times. The correlation length that obtained at different system size shows that the finite-size effect is still negligible because the data agree within the error bars. Therefore we can focus on smaller system size, $L=40 R_{\mathrm{e} 0}$ in the following.

The correlation length, $\xi(t)$, increases like a power law, $\xi(t) \sim t^{\eta}$ found by previous studies in experiments and continuum models. The growth exponent, $\eta$ ranges from 0.066 to $0.3[14,53,132-134]$.

In Fig. 4.7, we present the time evolution of the correlation $\xi(t)$ at $\chi N=15,16$, $17,18,19$, and 20 . The correlation length, $\xi$ at a given, specific simulation time, decreases with $\chi N$. In Fig. 4.1 (c), we find that the size of lamellar grains in each morphology gradually decreases with $\chi N$. This observation is consistent with the behavior of the correlation length. 


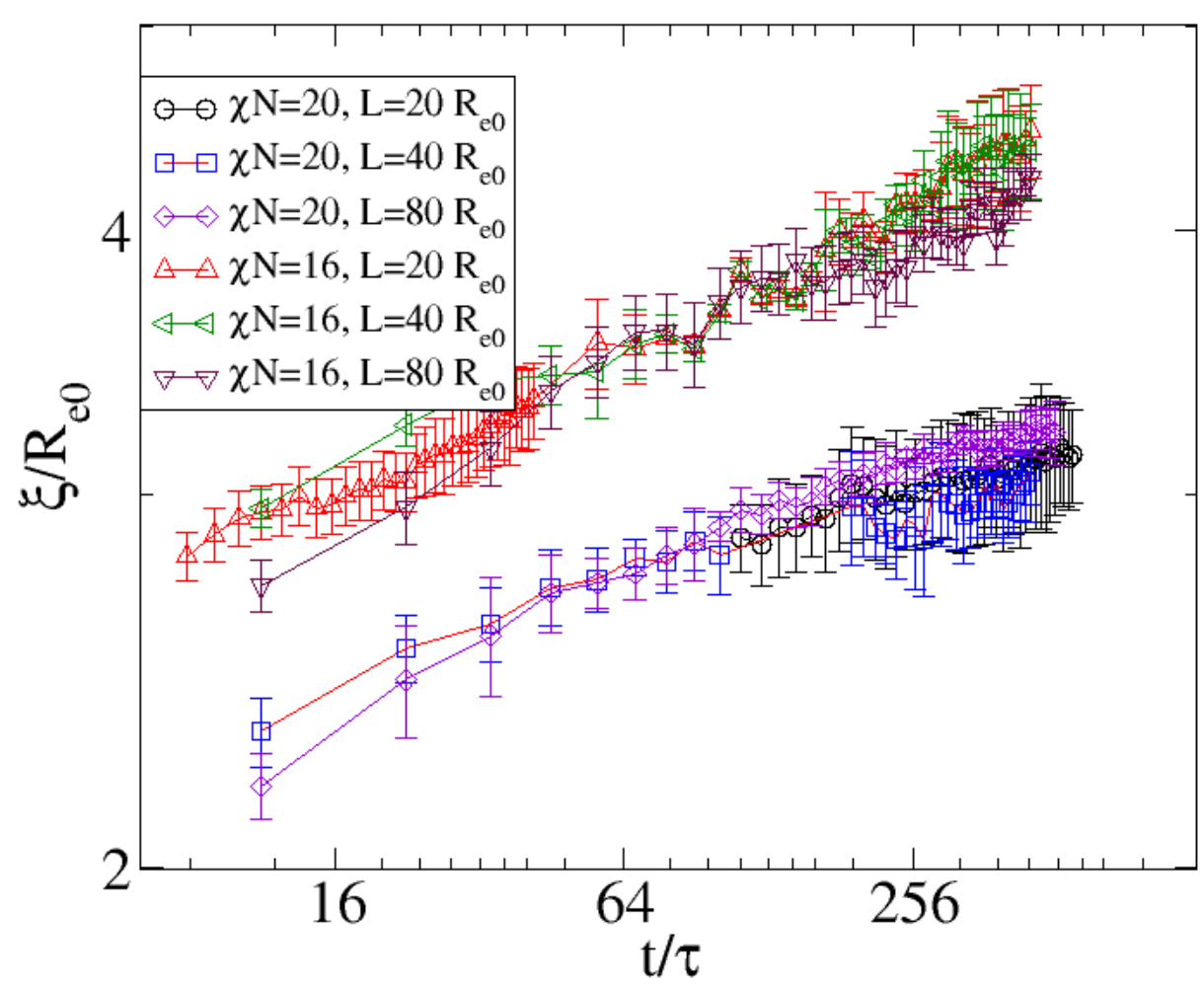

FIGURE 4.6: The correlation length, $\xi$ is plotted as a function of time, $t$. Results are obtained at $\chi N=16$ and $\chi N=20$ for different system size $L$. Each point on the graph is averaged over 5 independent simulations.

Due to the limitation of the current amount of computational resources, the simulation data can span only a limited regime. Our simulation time exceeds the smallest characteristic time, $\tau=8900$ only by about 2.5 orders of magnitude. The correlation length varies only by a factor of 2 when we use the smallest value of the growth exponent. It is insufficient to accurately extract growth exponents. We can, however, qualitatively observe that the initial growth for $t<10^{1} \tau$ is much faster than the later times after $t \approx 10^{1} \tau$. The growth exponent $\eta \approx 0.25$ and $\eta \approx 0.1$ are compatible with the simulation data and are illustrated in the figure which coincides with experimental results [14].

We assume that the growth exponent is a constant for $t>100 \tau$ to approximately extrapolate the ordering time of correlation length to reach a specified value. The extrapolations are presented in the main panel of Fig. 4.7. We present in the inset of the figure an Arrhenius plot of the extrapolated times, $t_{\xi}^{*}$, to reach the specified correlation lengths, $\xi=4 R_{\mathrm{e} 0}$ or $5 R_{\mathrm{e} 0}$, respectively. The extrapolated simulation are compatible with a thermally activated behavior, $t_{\xi}^{*} \sim \exp \left(\Delta F / k_{\mathrm{B}} T\right)$. The activation free energy is on the order of 


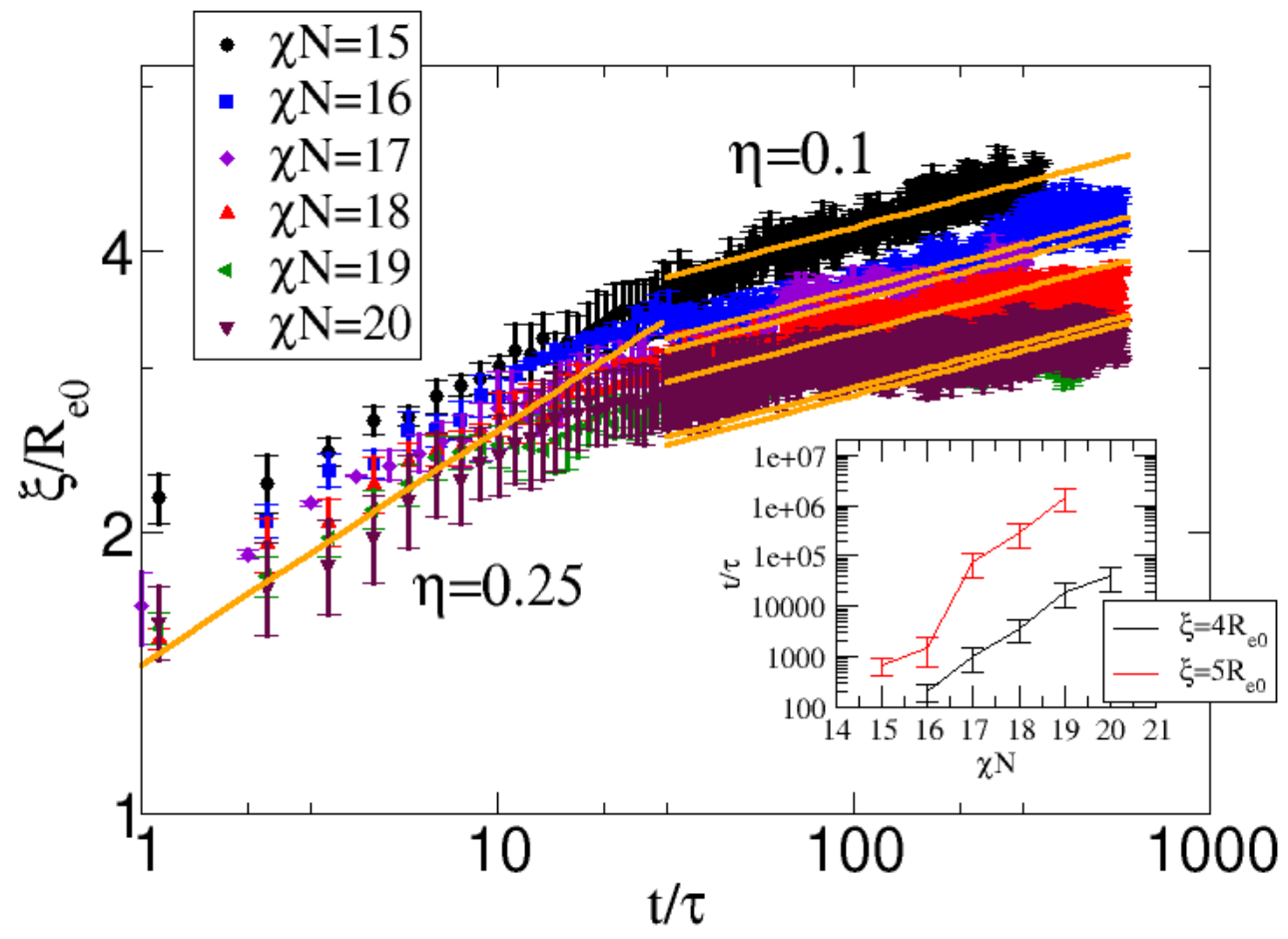

FIGURE 4.7: The correlation length $\xi$ is plotted as a function of simulation time $\mathrm{t}$ in unit $\tau$. The results are averaged over 5 independent simulations at $\chi N=15,16,17,18,19,20$. Inset: The extrapolated time for the system with different $\chi N$ to the correlation length $4 R_{e 0}$ and $5 R_{e 0}$.

$\Delta F / k_{\mathrm{B}} T \approx 1.45 \chi N$ and $2 \chi N$ for $\xi=4 R_{\mathrm{e} 0}$ and $5 R_{\mathrm{e} 0}$, respectively. Qualitatively, the linear dependence on $\chi N$ in the range $15 \leq \chi N \leq 20$ agrees with the excess free energy dependence of isolated dislocation pairs on $\chi N$ and their free-energy barrier, $\Delta F_{\mathrm{b}}$ for the defect annihilation in a perfectly ordered lamellar structure [130].

Our analysis is limited by the simulation time and statistic errors. However our simulation results, in conjunction with the visual impression of the snapshots in Fig. 4.1, indicate that the dependence on the incompatibility, $\chi N$, is rather gradual. Even at the largest value of $\chi N$, the coarsening process does not completely arrest but only becomes very protracted. 


\subsection{Identifying free-energy basins: Inherent morphol- ogy and Euler characteristics}

\subsubsection{Integral-geometry morphological analysis}

In the previous section, we observe that morphologies can be "frozen" at large $\chi N$, i.e., the topological structure of a morphology persists for several hundred $\tau$. It is crucial to investigate the change of topological structures because it is probably related to a free-energy basin hopping event.

We use the Euler characteristic, $\chi_{\mathrm{E}}$ as an indicator to monitor the change of topological structures. The Euler characteristic, $\chi_{\mathrm{E}}$ gives a unique integer value to a specific topological structure. The measurement of the characteristic is implemented by the integral-geometry morphological analysis method [135]. This method has been applied to classify disordered structures, e.g., to distinguish ripple- and dimple-like morphologies. If $\chi_{\mathrm{E}}$ is approximately zero, the pattern looks ripple-like. Otherwise, $\chi_{\mathrm{E}}>0$ gives a signal that there are many $A$ domains which are dispersed in a $B$ matrix and, conversely, a morphology with many isolated $B$ domains in a continuous $A$ matrix results in $\chi_{\mathrm{E}}<0$.

A two-dimensional pattern, according to integral geometry, is characterized by three, additive, translational and rotationally invariant, Minkowski measures $[135,136]$ : the area of $A$ domains, $A_{s}$, the perimeter of $A$ domains, $U$, and the Euler characteristic, $\chi_{\mathrm{E}}$, which equals to the number of $A$ domains minus the number of holes surrounded by $A$ domains.

In the particle-based simulations, our calculation is implemented via the densities on a collocation grid. We obtain a discretized, two-dimensional orderparameter field by averaging the density along the thin $z$-dimension of the film. If the $z$-averaged order parameter is positive, we assign the value +1 to the grid cell, and 0 otherwise. we use the composition of two species, $m(\vec{r})$ as the order parameter. This continuum variable is discretized by the first-order assignment function, $\Pi(\mathbf{c}, \vec{r})$ defined in Eq. 2.23.

Now the order parameter, $m(\mathbf{c})$ is three dimensional. To obtain a two-dimensional description of the morphology, we average the order parameter $m(\mathbf{c})$ over the 
configuration which is perpendicular to the morphology,

$$
m_{2 \mathrm{~d}}\left(\mathbf{c}^{\prime}\right) \equiv \sum_{x} m(\mathbf{c}) / N_{x}
$$

$N_{x}$ is the number of grids on the x direction.

$m_{2 \mathrm{~d}}\left(\mathbf{c}^{\prime}\right)$ is a grey-level, two-dimensional function. We replace the intensity of the pixel $m_{2 \mathrm{~d}}\left(\mathbf{c}^{\prime}\right)$ by a black or a white pixel $\mathcal{P}\left(\mathbf{c}^{\prime}\right)$,

$$
\mathcal{P}\left(\mathbf{c}^{\prime}\right)= \begin{cases}1 & m_{2 \mathrm{~d}}\left(\mathbf{c}^{\prime}\right)>=0 \\ 0 & m_{2 \mathrm{~d}}\left(\mathbf{c}^{\prime}\right)<0 .\end{cases}
$$

If the number of $\mathrm{A}$ segments dominates in the square pixel $\mathbf{c}^{\prime}, \mathcal{P}\left(\mathbf{c}^{\prime}\right)=1$, otherwise $\mathcal{P}\left(\mathbf{c}^{\prime}\right)$ gives a value of 0 .

We can straightforwardly obtain the Minkowski measures from this discretized, two-dimensional, binary map of the system [135]. Each pixel of this map is a square-shaped region with 4 vertices and 4 edges. We colored the interior of the pixel according to the binary map. For each $A$-rich pixel, we define $n v$ as the total number of vertices, ne as the total number of edges, and $n s$ as the total number of $A$-rich pixels. Counting these values over all $A$-rich pixels, we obtain the Minkowski functionals as [135],

$$
\begin{aligned}
A_{s} & =n s \\
U & =-4 n s+2 n e \\
\chi_{\mathrm{E}} & =n s-n e+n v .
\end{aligned}
$$

The time evolution of the area $A_{s}$ of $A$ domains and length $U$ of the internal $A B$ interfaces are illustrated in Fig. 4.8 for a system of lateral size $L=8 R_{\mathrm{e} 0}$ in response to a quench to $\chi N=30$. The fluctuations are induced by the assignment of the $z$-averaged order-parameter profile to the binary map. At the initial time, the length $U$ which indicates the internal $A B$ interfaces decreases with time. $U$ gradually decreases when the morphology becomes more ordered, Eventually, the value measured in the simulation is still larger than the ideal value $U^{*} \approx 2 L^{2} / L_{\mathrm{s}} \approx 70$, where $L_{\mathrm{s}}$ is the lamellar spacing, of a perfect, lamellar structure. 


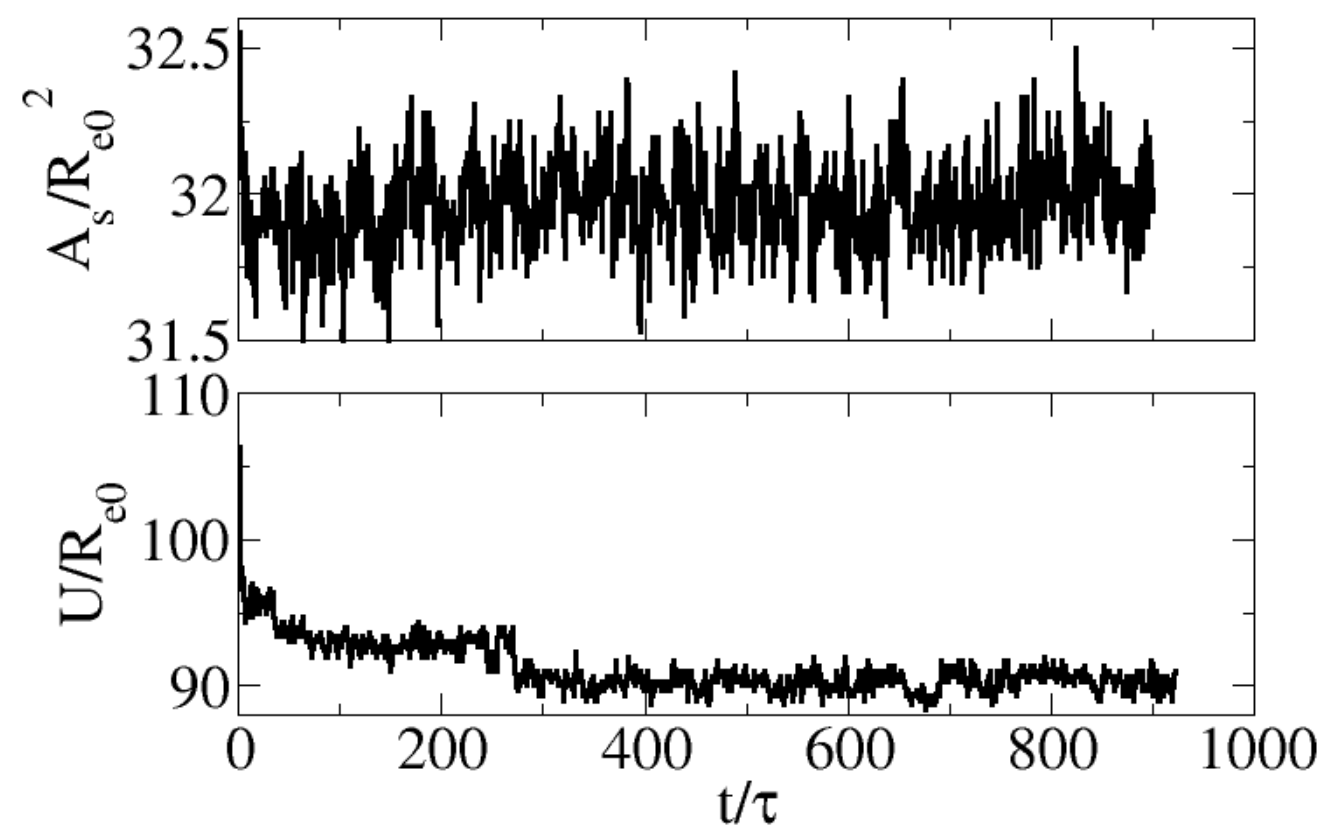

FIGURE 4.8: Upper: The A domain area is plotted as a function of time $t$. Lower: The time evolution of the perimeter of A domain as a function of simulation time $t$.

\subsubsection{Identifying free-energy basins}

Previous results indicate that the ordering of diblock copolymers in a thin film consists of a sequence of thermally activated processes, which can be conceived as "jumps between free-energy basins". In a self-assembly fluid, free-energy basins are difficult to be directly identified because we cannot simply minimize the potential energy by a rapid temperature quench $T \rightarrow 0$. As a contrast, the glass-forming liquids of hard-core particles characterize the basin by its corresponding minimum-energy configuration. In a self-assembling fluid, the individual molecules diffuse even in the perfectly ordered state. We need to identify a local free-energy minimum that comprises a region in configuration space instead of a single minimum-energy configuration.

To characterize a free-energy basin, we employ the order parameter, $m(\vec{r})$, to 
describe the morphology, instead of utilizing the coordinates of individual particles. The advantage of this collective order parameter is that it remains unchanged under permutations of the same kind of particles, as a result, this order parameter automatically accounts for the indistinguishability of identical particles. It also remains the domain structure when the molecules of the liquid diffuse.

According to above discussions, a free-energy basin is quantitatively characterized by a local minimum of the free-energy landscape, $F[m]$. A metastable state can be well defined within SCFT because it ignores fluctuations of the order parameter, $m$. Initialized with the morphology observed in the particle-based simulation, which includes fluctuations, we use the SCFT calculation to converges to the nearest, local free-energy minimum. The free-energy basin of the initial particle-based configuration is characterized by this metastable state. The limit of $\overline{\mathcal{N}} \rightarrow \infty$ in SCFT, corresponds to the limit, $1 / T \rightarrow \infty$ in the hard-core particles when minimizing the potential energy in a glass in order to identify inherent structures. In this thesis, the so-obtained free-energy minima are referred to as inherent morphologies.

In our case, the soft, coarse-grained model can be directly mapped onto the standard model of SCFT [137]. So this strategy to identify free-energy basins is reliable because the SCFT method is an accurate and numerically tractable mean-field theory for the particle-based model. However, an explicit form of the free-energy functional, $F[m]$ for a particle-based model is not known. In order to identify the local minima, it is useful to identify other indicators.

We qualitatively assume that the change of free-energy basin is related to the breaking or connecting of domains. Meanwhile, a change of domain connectivity results in a discrete change of the Euler characteristic of the domain structure. In the particle-based simulations, we observe that domain topology remains unaltered for several hundred $\tau$. Details about the computation of the Euler characteristic are presented in the previous section. The Euler characteristic can be straightforwardly applied in our simulation data and experimental studies of self-assembly.

To illustrate the relation between inherent morphologies and Euler characteristic, we will present two examples obtained in the particle-based simulations. We study the time evolution in a quasi-two-dimensional system with $L=20 R_{\mathrm{e} 0}$ 
(a)

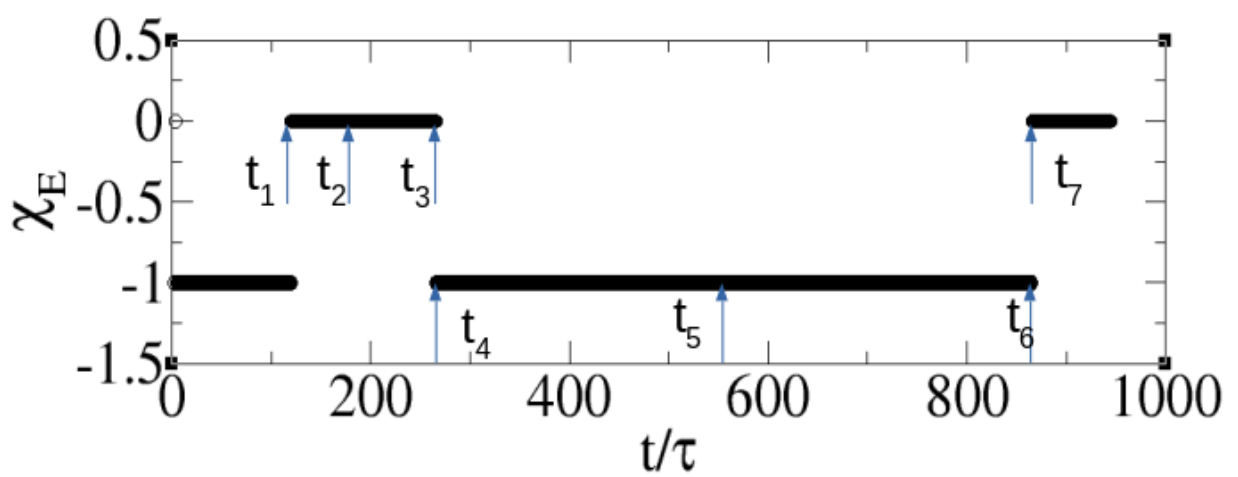

(b) Particle-based simulations
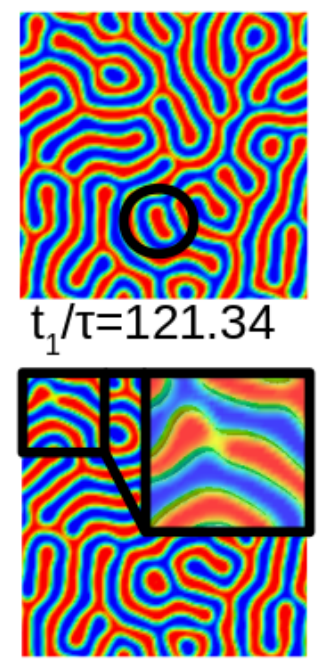

$\mathrm{t}_{4} / \mathrm{\tau}=266.29$

$20 R_{e 0}$

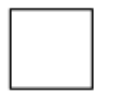

$6 R_{e 0}$
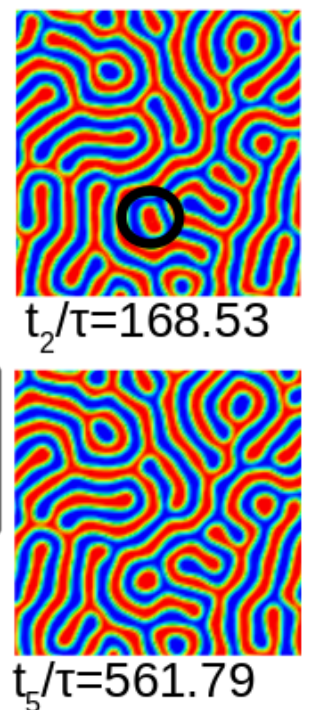
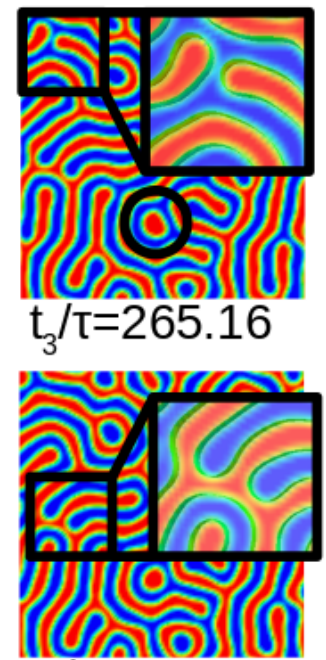

$\mathrm{t}_{6} / \mathrm{t}=866.29$

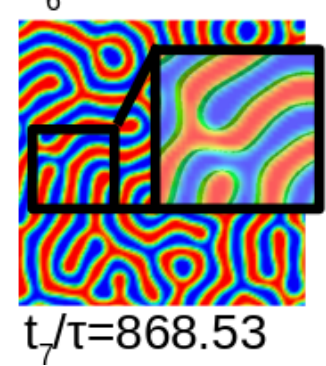

(c) SCFT

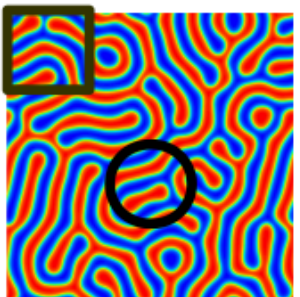

$(\alpha)$

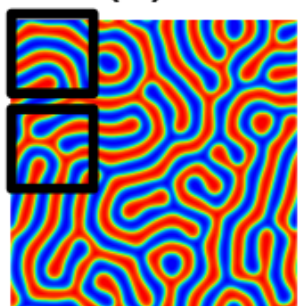

$(\beta)$

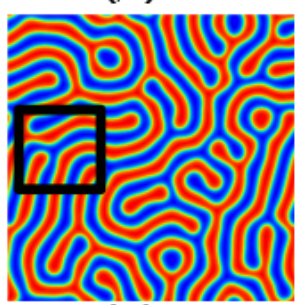

$(\gamma)$

FIGURE 4.9: (a) Euler characteristic, $\chi_{\mathrm{E}}$ is plotted as a function of time $t$ at $\chi N=30$. (b) Snapshots of particle-based simulations. Times are indicated by arrows in panel (a). The black circle indicates the position, where we can observe that the size of an isolated domain varies in time. We use squares to highlight areas where changes of the domain topology occur. Enlarged portions of the domain structure are shown in the inset. Two ending $A$ domains connect between $t_{3}$ and $t_{4}$. one lamellar domain breaks from $t_{6}$ to $t_{7}$. (c) Inherent morphologies: All instantaneous morphologies of the particle-based simulation at $t_{1}, t_{2}$ and $t_{3}$ correspond to the same inherent morphology $\alpha$. The inherent morphology $\beta$ is obtained by quenching the instantaneous particle-based morphologies at $t_{4}, t_{5}$ and $t_{6}$, whereas the inherent morphology $\gamma$ characterizes the free-energy basin at $t_{7}$. Squares on the figure indicate the position, where changes of the domain topology occur which corresponds to jumps from one free-energy basin to another. 
and the thickness of the system is $D=R_{\mathrm{e} 0}$, at $\chi N=30$. We show the first prototypical example in Fig. 4.9 (a). The changes of the Euler characteristic are identified at times, $t \approx 119 \tau, 264 \tau$, and $866 \tau$. We present the concomitant snapshots of the morphology in panel (b) and times are marked by arrows in panel (a).

At $t \approx 264 \tau$, we observe that two $A$ domains, which are colored red, connect. As a result, the Euler characteristic, $\chi_{\mathrm{E}}$, decreases from 0 to -1 . One lamellar domain breaks at time $t \approx 866 \tau$ and $\chi_{\mathrm{E}}$ increases from -1 to 0 . The typical time difference between changes of the domain topology can exceed several hundred $\tau$ at the large incompatibility, $\chi N=30$.

To test the assumption that the change of topology is related to a jump between free-energy basins, we employ the SCFT method. The instantaneous density from the the particle-based simulations at times $t_{1}=121.34 \tau, t_{2}=168.53 \tau, t_{3}=$ $265.16 \tau, t_{4}=266.29 \tau, t_{5}=561.79 \tau, t_{6}=866.29 \tau$, and $t_{7}=868.53 \tau$, are averaged over the short $z$-direction. We construct a two-dimensional order-parameter field, $\bar{m}$ on a discretized, $480 \times 480$ square grid. We utilize these two-dimensional fields as the initial condition for SCFT. In the end, these SCFT calculations will converge to the nearest free-energy minimum, i.e., the corresponding inherent morphology. Thermal fluctuations of the order parameters are eliminated.

In Fig. 4.9 (b) and (c), we find that morphologies in the particle-based simulation and the inherent morphologies have identical domain topologies. Morphologies at $t_{1}, t_{2}$, and $t_{3}$ have identical inherent morphologies and denoted by $\alpha$ in panel (c). Similarly, the inherent morphologies that correspond to times $t_{4}, t_{5}$, and $t_{6}$ are also identical. They are in the same free-energy basin that is characterized by the inherent morphology $\beta$. The particle-based simulation is in the free-energy basin of the inherent morphology $\gamma$ at $t_{7}$. This example shows that changes of the Euler characteristic (i.e., the change of domain topology) are highly associated with jumps between free-energy basins, which are characterized by inherent morphologies.

In the SCFT calculation, we can directly obtain the free energy to characterize the inherent morphologies. The free-energy difference between inherent morphologies $\alpha$ and $\beta$ is $\Delta f=0.0020734 k_{\mathrm{B}} T$ per chain in the two-dimensional SCFT calculations, which corresponds to $\Delta F=\sqrt{\mathcal{\mathcal { N }}} \frac{V}{R_{\mathrm{e} 0}{ }^{3}} \Delta f \approx 106 k_{\mathrm{B}} T$ in the quasitwo-dimensional system studied in the particle-based simulation. Similarly, 
from the inherent morphology $\beta$ to $\gamma$, free energy reduces by $\Delta F \approx 70.6 k_{\mathrm{B}} T$. At the same incompatibility, $\chi N=30$, the excess free-energy of apposing dislocation defects, $F_{\mathrm{d}} \approx 229 k_{\mathrm{B}} T$ is about three times of these free-energy changes [130]. For polymeric systems with a large degree of polymerization, $\overline{\mathcal{N}}$, such large free-energy changes are typical, because the changes of the domain topology involve the participation of many molecules which strongly interdigitate.

Previous investigations suggest that morphologies located inside the same freeenergy basins, include thermal fluctuations around the inherent morphology. By observations of instantaneous snapshots of the particle-based simulations, we find that the morphology also evolves deterministically towards the inherent morphology, which is driven by long-range strain-field mediated PeachKoehler forces [138] and compression forces [139] that alter when the domain topology is changed. This deterministic evolution towards the inherent morphology can be protracted, because it may involve co-operative rearrangements on the scale of multiple lamellae, induced by long-range strain fields. Especially at early times, the time scale of the collective relaxation of the domain structure towards the inherent morphology and the thermally activated jumps from one free-energy basin to another may not separate.

Such a protracted, gradual change example is shown in Fig. 4.9 (b). We highlight an enclosed, isolated $A$-domain by the circle, whose size is on the order of $2 R_{\mathrm{e} 0}$. The morphologies of the particle-based simulation at $t_{1}, t_{2}$ and $t_{3}$ correspond to the same inherent morphology $\alpha$. However, we note that this enclosed, isolated $A$ domain at at $t_{1}, t_{2}$ and $t_{3}$ differs in size, shape, and orientation, even after the time $\Delta t=140 \tau$. In this particular case, the morphology in the particle-based simulation did not completely relax towards the inherent morphology. The relaxation time, $\Delta t$ significantly exceeds the time scale for a copolymer to diffuse parallel to the internal $A B$ interface of the $A$ domain. Such a protracted relaxation process is caused by two factors: (i) It is an intrinsically slow process to change the size of an enclosed $A$ domain [110] because this process involves the transfer of $A$ blocks across the enclosing $B$ domains, which incurs a large free-energy barrier onto the single-chain dynamics [139]. This process is also similar to the perpendicular diffusion of copolymers in a perfectly ordered lamellar structure from one lamella to a neighboring one [140, 141]. (ii) The change of the orientation of the $A$ domain also involves modifications of neighboring domains. 


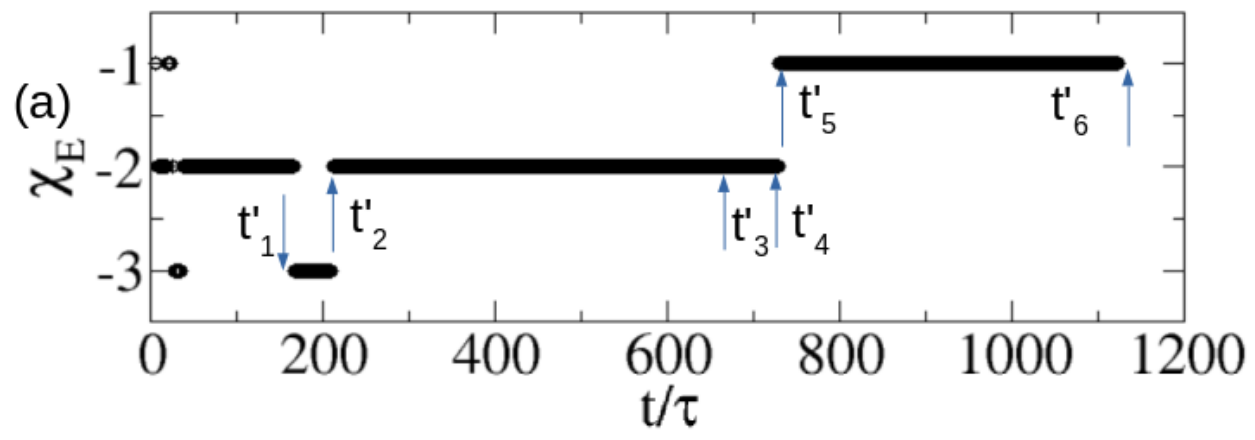

(b) Particle-based simulations
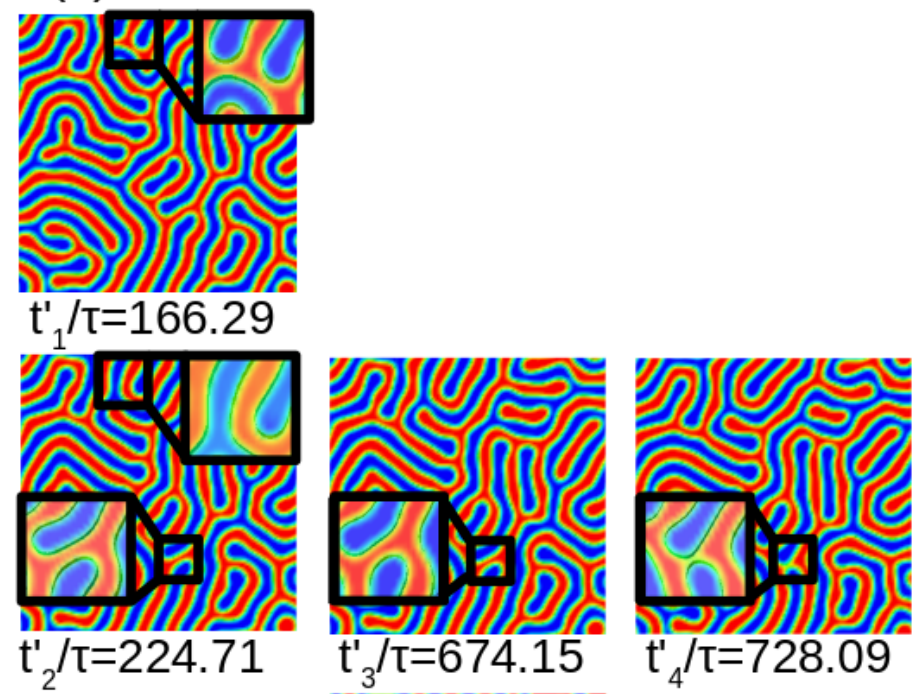

(c) SCFT

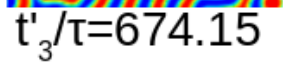

$t_{4}^{\prime} / t=728.09$

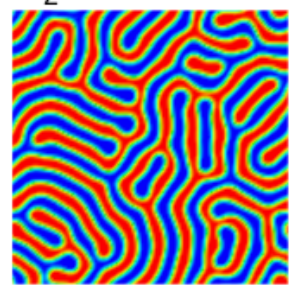

$\mathrm{t}_{5} / \mathrm{\tau}=731.46$
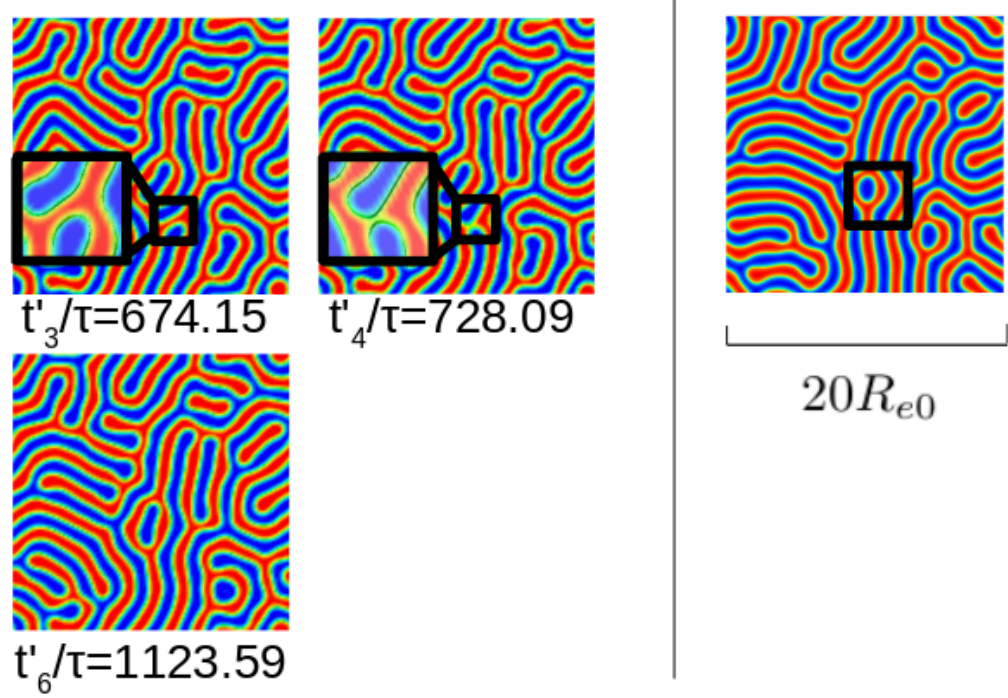

$20 R_{e 0}$

FIGURE 4.10: (a) The Euler characteristic, $\chi_{\mathrm{E}}$, as a function of time at $\chi N=30$. (b) Snapshots of particle-based simulations at times that are marked by arrows in panel (a). One lamellar connection breaks between $t_{4}^{\prime}$ and $t_{5}^{\prime}$; this breaking event is presented in the enlarged insets. (c) Inherent morphology that characterizes the free-energy basin corresponds to all simulation morphologies at $t_{1}^{\prime}$, $t_{2}^{\prime}, t_{3}^{\prime}, t_{4}^{\prime}, t_{5}^{\prime}$, and $t_{6}^{\prime}$. 
Due to the lack of time-scale separation between the relaxation of a morphology inside a free-energy basin towards the inherent morphology and subsequent jumps between free-energy basis, it is possible that false identifications of jumps between free-energy basins by changes of the Euler characteristic, occur. The change of the Euler characteristic will overestimate the rate of basin jumps. When the domain-breaking and connecting events occur on a time scale that is comparable to the time between basin jumps, the multiple changes of the Euler characteristic and a basin-jump are not bunched together.

As shown in Fig. 4.10, we present such a case that the change involves multiple events of domain breaking and connecting. In panel (a) of Fig. 4.10, three changes of the Euler characteristic of the domain morphology are observed around $t_{1}^{\prime} \approx 166 \tau, t_{2}^{\prime} \approx 225 \tau$, and $t_{4}^{\prime}=728 \tau$. Similar to the previous example, various instantaneous snapshots of the particle-based simulation are obtained. In panel (b), the corresponding inherent morphologies are presented. We find that all snapshots of the particle-based simulation result in the same inherent morphology which is shown in panel (c). The simulation remains located inside the same free-energy basin, after the $\chi_{\mathrm{E}}$-change at $t_{1}^{\prime} \approx 225 \tau$. After $t_{1}^{\prime} \approx 225 \tau$, the domain topology of the particle-based simulation does not coincide with the domain topology of the inherent morphology for $\Delta t \approx 600 \tau$. During this simulation time, we observe that the lamellar domain, which is highlighted in panel (b), gradually becomes thinner. At around $t_{4}^{\prime} \approx 787$ after this lamellar domain breaks, the domain topology of the simulation coincide with the domain topology of the inherent morphology.

In the subsequent process, the morphology continues to evolve in response to the remaining strain field even after $\Delta t=336 \tau$ (at $t_{6}^{\prime}=1123 \tau$ ). At the end of the simulation, the morphology still differs from the inherent morphology not only by thermal undulations of the internal $A B$ interfaces.

By this caveat, we try to quantify the rate with which the domain topology changes in the course of structure formation in the particle-based simulation. We measure the fraction, $\Pi_{\mathrm{E}}\left(t, t_{e}\right)$, of the self-assembling process that change their Euler characteristics between the time points, $t-\Delta t_{\mathrm{E}}$, and $t$.

$$
\Pi_{\mathrm{E}}\left(t, \Delta t_{\mathrm{E}}\right)=\frac{1}{N_{s}} \sum_{k=1}^{N_{s}} \bar{\Theta}\left(\chi_{\mathrm{E}}^{k}(t)-\chi_{\mathrm{E}}^{k}\left(t-\Delta t_{\mathrm{E}}\right)\right)
$$




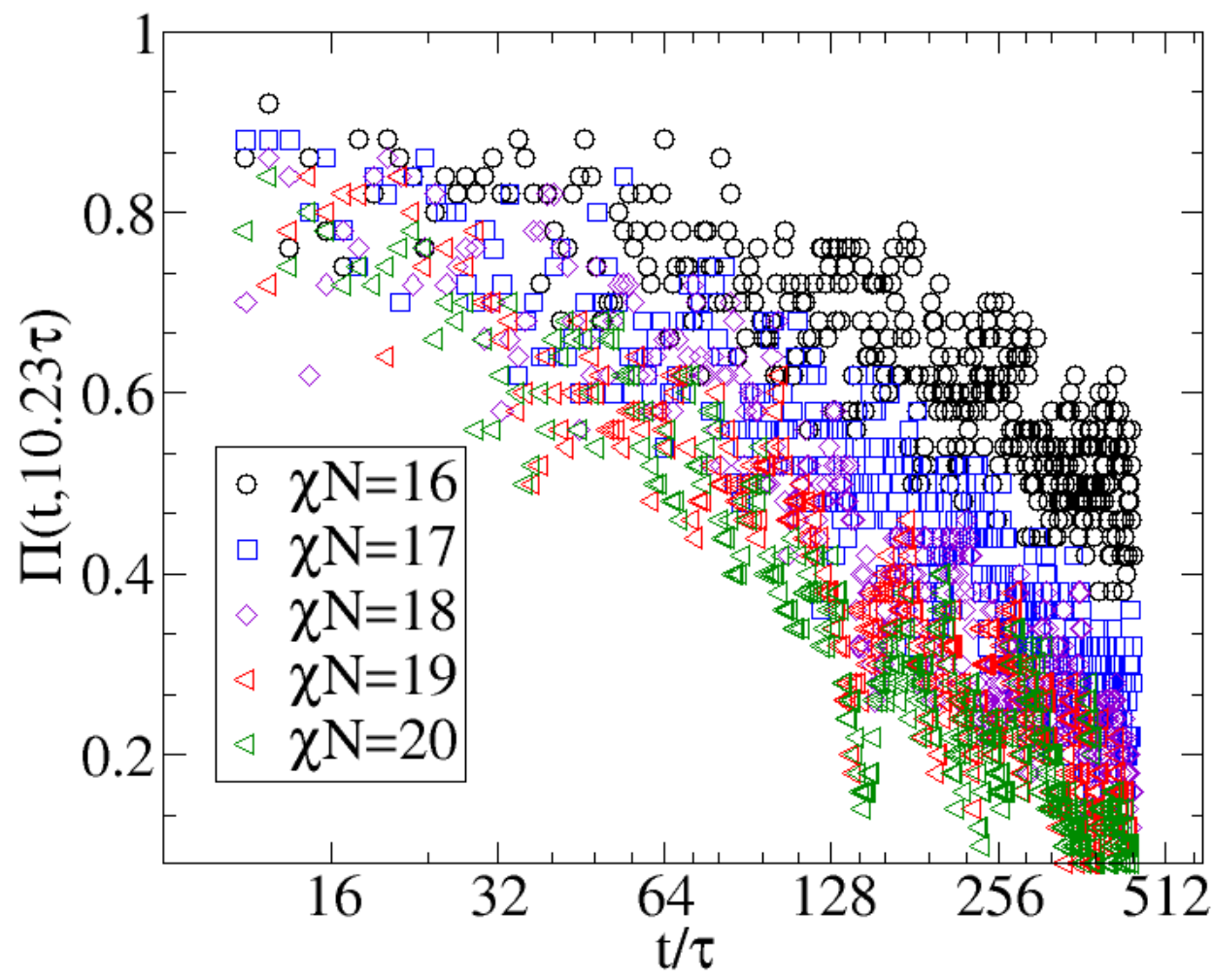

FIGURE 4.11: The persistence rate of Euler characteristic $\Pi(11.23 \tau)$ is presented as a function of the simulation time, $t$ at $\chi N=16,17,18,19,20$.

where $k=1, \cdots, N_{s}=50$ loops over all $N_{s}$ independent simulations at a given incompatibility, $\chi N$. The variable $\bar{\Theta}=1$ if the argument is non-zero, and $\bar{\Theta}(0)=$ 1 . In the following, we use a large time interval, $\Delta t_{\mathrm{E}}=11.23 \tau$ or 100000 MonteCarlo steps, which is larger than the actual time to break or connect a domain, and it allows for some relaxation of the morphology.

In Fig. 4.11, we plot the probability of changing the Euler characteristics during the time interval $[t-11.23 \tau: t]$ for various incompatibilities, $\chi N$. The size of the system is $20 R_{\mathrm{e} 0} \times 20 R_{\mathrm{e} 0} \times R_{\mathrm{e} 0}$. At a given incompatibility, $\Pi_{\mathrm{E}}$ decreases with time. The time dependence of the simulation data is compatible with an exponential behavior, which give rises to a characteristic time, $\tau_{\Pi}$ that decreases with incompatibility from about $800 \tau$ at $\chi N=16$ to about $250 \tau$ at $\chi N=20$. This is consistent with our previous qualitative impression of the snapshots in Fig. 4.1 that the time evolution is significantly faster at lower incompatibility and there is a pronounced but gradual slowing down of the ordering kinetics. 


\subsection{Defects}

The change of free-energy basins, which involves the breaking or connecting of domains is also a defect annihilation process. In a stripe phase environment, the annihilation of isolated defects (e.g., the annihilation of disinclination quadrupole or the annihilation of disinclination dipole) is well-understood [28]. In a stripe pattern with two or four disclinations on the film, two disclinations are attracted by a driving force which is proportional to their inverse distance, i.e., $1 / r$. Peach and Koehler generalize this force and develop Peach-Koehler equation to describe the force of dislocation from the internal stress [142].

In a more disordered morphology, the dynamics of defect annihilation are rather co-operative and are strongly influenced by surrounding defects. In the subsequent section 4.5.3.1, we find one defect which is similar to the B-core dislocation dipole defect but in a disordered environment. In the particle-based simulation, the defect annihilation process is rather different from the process in a lamellar phase.

It is, therefore, necessary to understand the defect annihilation processes, statistically. Motivated by experiments and numerical approaches [28, 126, 143], we measure the kinetic exponents in terms of defect annihilation process.

\subsubsection{Approximately estimating the defect density via the Euler characteristic of small patches}

In this section, we will introduce a scheme that allows us to estimate and approximately categorize defect motifs in a two-dimensional morphology by distinguishing topological structures of A domains. A typical simulation snapshot is depicted in Fig. 4.12, featuring different kinds of local defect motifs: an isolated domain, an end of a lamellar domain, and an intersection of two lamellar domains. These local defect motifs differ in their topology and these distinctions can be quantified by the Euler characteristic, $\chi_{\mathrm{E}}$. We compute the Euler characteristic, $\chi_{\mathrm{E}}$, of $\mathrm{B}$ domains (blue color) in a small square detection region, as illustrated in Fig. 4.12. For the isolated domains, $\chi_{\mathrm{E}}$ gives a value of 0 . Similarly, $\chi_{\mathrm{E}}$ is 1 for the end of a lamellar domain and the intersection of domains 


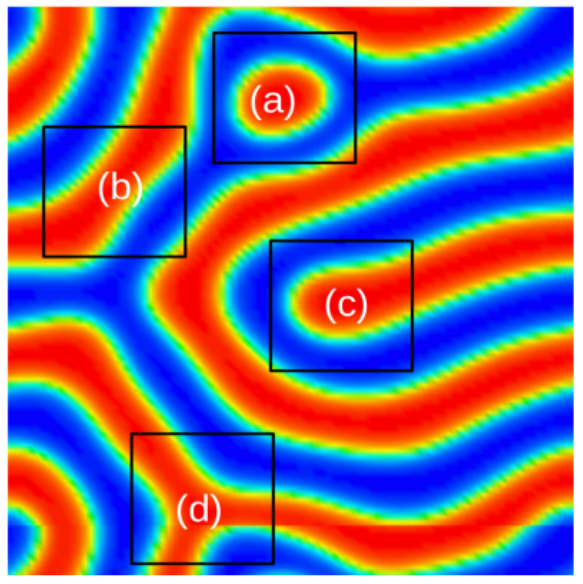

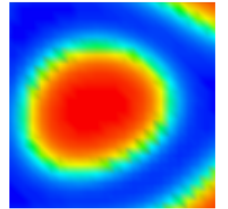

(a) Isolated block

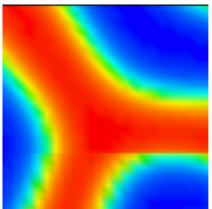

(c) Intersection

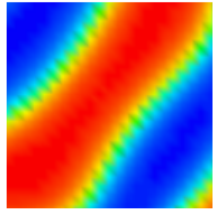

(b) lamella

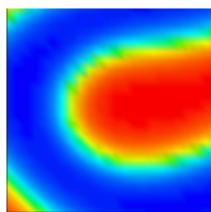

(d) End of the line

FIGURE 4.12: Left is the snapshot of one typical morphology obtained by the particle-based simulation. This morphology contains three typical defects which are marked by a square curve. On the right, we present these defects in the detection region.

results in a value of 3 . A defect-free structure in the detection region yields the value of $\chi_{\mathrm{E}}=2$.

We move the small square detection region, which is centered in A domains across the two-dimensional morphology. A defect is detected when the Euler characteristic, $\chi_{\mathrm{E}}$ do not take the value of 2 . We have two difficulties in practice, which would possibly induce a false signal of defects.

The first difficulty is to determine a proper size of the detection region to reduce the influence of irrelevant structures. Fortunately, in diblock copolymer melts, the lamellar spacing is determined by the Flory-Huggins parameter $\chi N$, which gives a uniform width of the stripe in the morphology. This property guarantees that a proper size of the detect region exists.

To determine a proper size of the detection region, $L_{\mathrm{d}} \times L_{\mathrm{d}}$, we measure the Euler characteristic of various defects with various values of $L_{\mathrm{d}}$. In Fig. 4.13, we measure the Minkowski functionals of B domains. We plot the Euler characteristic, $\chi_{\mathrm{E}}$ and the ratio of the area, $\mathrm{S}$, which is defined as the ratio of $\mathrm{B}$ domain in the detection region as a function of size of the detection region, $L_{\mathrm{d}}$.

These defects are placed at the center of the detect region. As shown in Fig. 4.13, the value of $L_{\mathrm{d}}$ ranges from $1 / 3 R_{\mathrm{e} 0}$ to $6 R_{\mathrm{e} 0}$. For the isolated block defect, there is window ranging from $L_{\mathrm{d}} \approx 1.5$ to $L_{\mathrm{d}} \approx 2.5$, where $\chi_{\mathrm{E}}$ gives the correct value of the defect. The area of $\mathrm{B}$ domains also reaches a peak in that window. From 

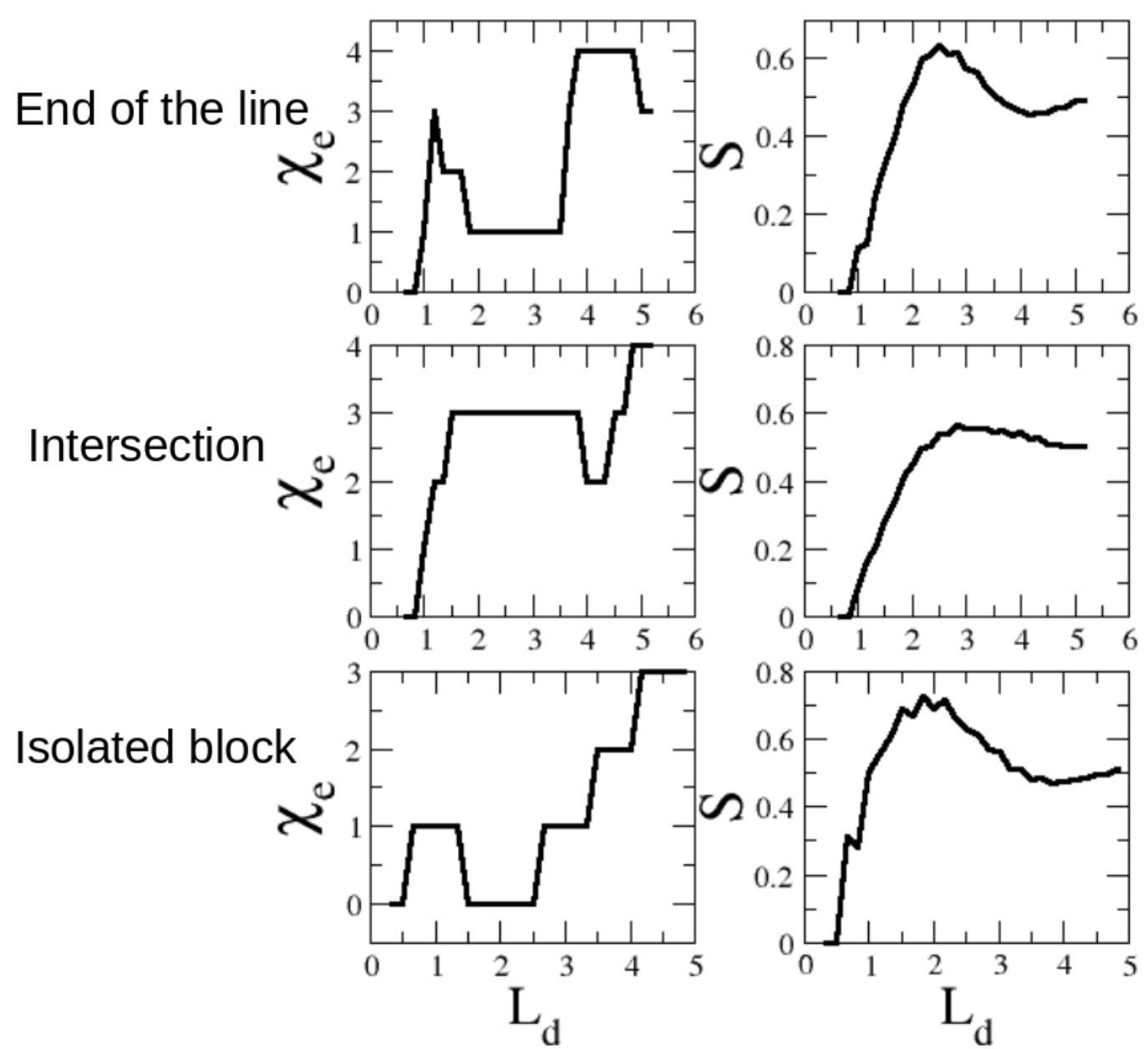

FIGURE 4.13: For different defects in the snapshot in Fig. 4.12, we plot the Euler characteristic $\chi_{\mathrm{E}}$ of the $\mathrm{B}$ domains (blue color) and the portion of $\mathrm{B}$ domains (blue area) in the detection region (right) as a function of the size of $L_{\mathrm{d}}$. Upper: the end of the line defect. Middle: the intersection defect. Lower: the isolated block defect.

this result, the proper size of the detection region ranges from $L_{\mathrm{d}}=1.5 R_{\mathrm{e} 0}$ to $L_{\mathrm{d}}=2.5 R_{\mathrm{e} 0}$. In the following, we use the value of $L_{\mathrm{d}}=2 R_{\mathrm{e} 0}$.

The second difficulty is to distinguish an end of a lamellar domain from the isolated block domain. The reason for this incorrect detection is that when the detection region is at the edge of an isolated block domain, the Euler characteristic, $\chi_{\mathrm{E}}$ also takes the value of 1, which corresponds to the end of a lamellar domain. This mistake is avoided by detecting different types of defects subsequently. We identify the isolated block domains first and mark the regions where the isolated block domains are detected. In the next round of defect detection, we start to detect the end of the line domains and do not take into 


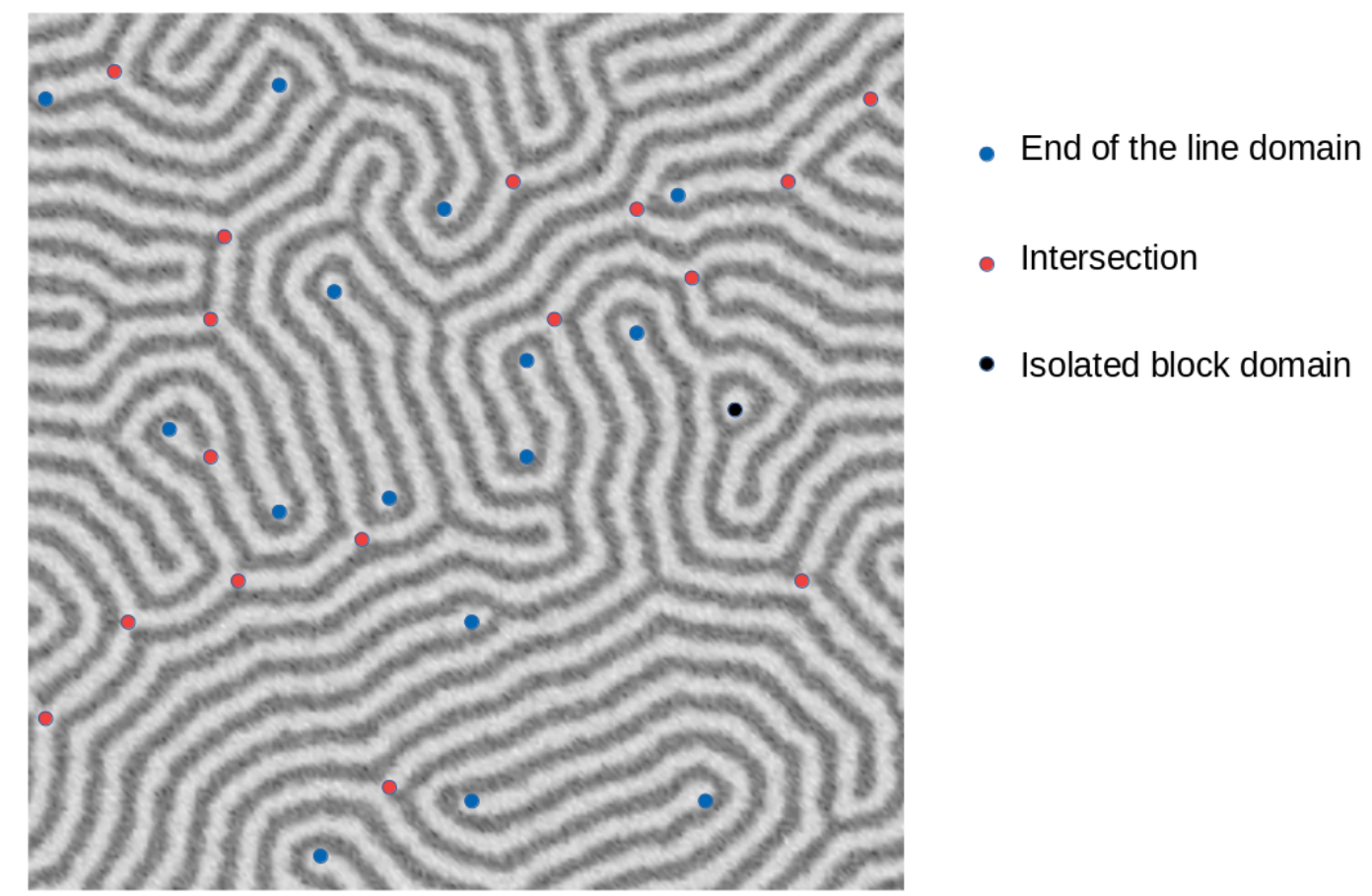

FIGURE 4.14: Defects in a typical two-dimensional fingerprint morphology obtained by the particle-based simulation which is quenched to $\chi N=20$.

account the regions where the isolated block domains are found.

To verify the algorithm, we count defects on a morphology with an extension $40 R_{\mathrm{e} 0} \times 40 R_{\mathrm{e} 0}$. The morphology is obtained by quenching the particle-based simulation to $\chi N=20$ and running the simulation for approximately $561 \tau$. As shown in Fig. 4.14, there are 15 end of line domains (blue points) and 16 intersection domains (red points). The is only one isolated block domains (black point). This result is verified by our algorithm.

\subsubsection{Quantifying the ordering process: time evolution of de- fect density}

The statistics of defect annihilation can be quantified by the time dependence of the number, $N_{\mathrm{d}}$ of defect. A description of the identification and classification of defect motifs is given in the previous section. In fig. 4.15, we present the density of defect motifs, $\rho_{\mathrm{d}}=N_{\mathrm{d}} / L^{2}$, in the data for various system sizes demonstrates the absence of finite-size effects, the data are compatible with a power-law decay of the defect density, $\rho_{\mathrm{d}} \sim t^{-\eta_{\mathrm{d}}}$ with an effective exponent, $\eta_{\mathrm{d}} \approx 0.2$. 


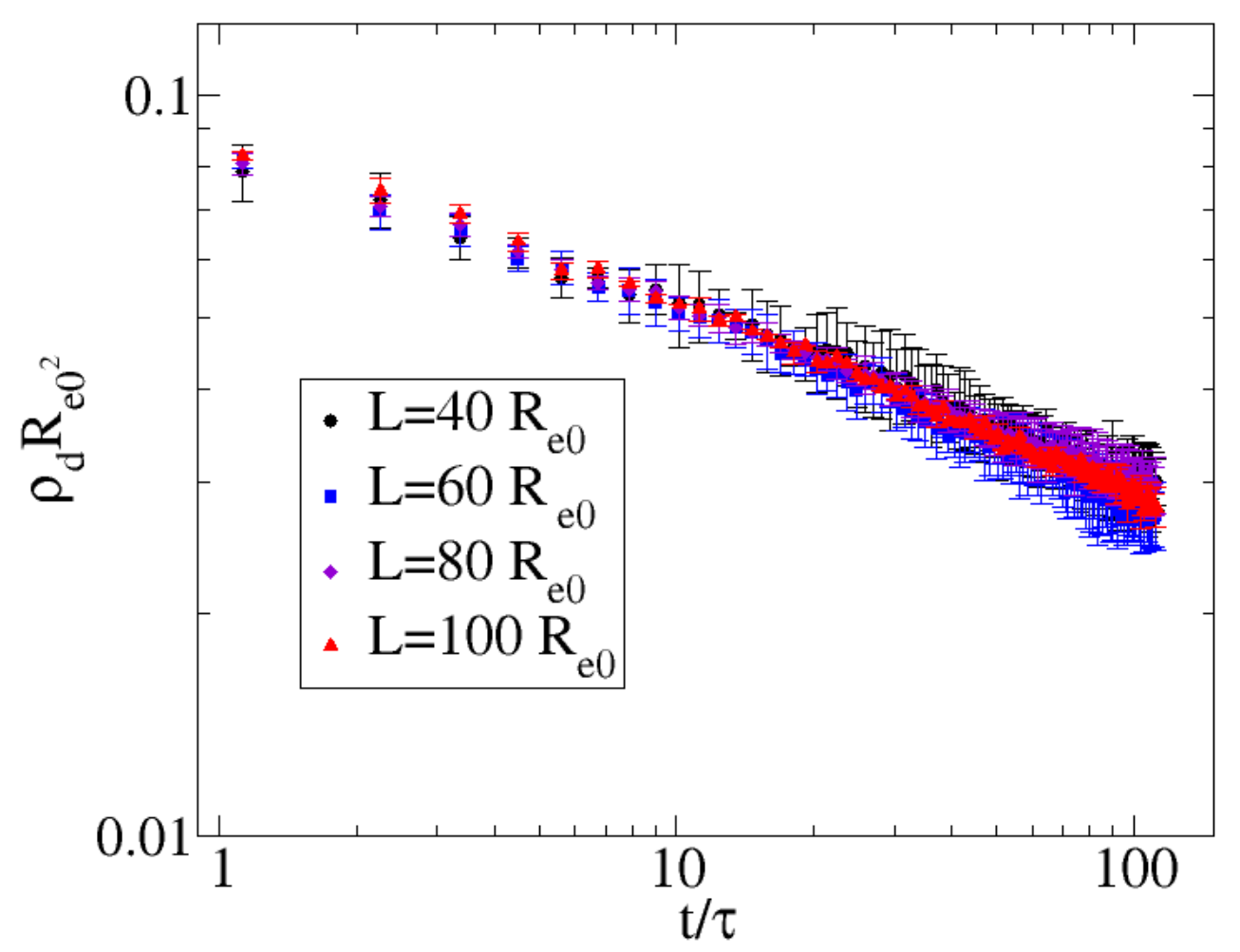

FIGURE 4.15: Estimate of the density of local defect motifs as a function of time $t$ after a quench to $\chi N=20$. The system size $L$, ranges from $40 R_{\mathrm{e} 0}$ to $100 R_{\mathrm{e} 0}$. Results are averaged over five independent simulations.

As shown in Fig. 4.15, we also measured the decrease of the defect density for other value of incompatibility, $\chi N$, using the system size, $L=40 R_{\mathrm{e} 0}$. In accord with the previous observations, the defect density at a given time increases with $\chi N$ and the exponent $\eta_{\mathrm{d}}$ decreases from $\eta_{\mathrm{d}} \approx 0.32$ to 0.02 as we increase $\chi N$ from 18 to 30 .

The local defect morphology can be classified by end of lamellar domains, intersections of domains, and isolated domains. In a symmetric copolymer system, the former two are equally probably within the statistical accuracy. The number of isolated domains-denoting small unconnected domains-is much smaller than the number of the former defects, e.g., for $L=100 R_{\mathrm{e} 0}, \chi N=20$, and $t=500 \tau$, the system contained 205 defects, of which only 3 were isolated domains. The number of end of the line defect and the intersection defect were 104 and 98, respectively.

The density of isolated-domain defects in the early stage of self-assembly is presented in Fig. 4.17 for $L=100 R_{\mathrm{e} 0}$. We observe that for $\chi N=18$ and 20, 


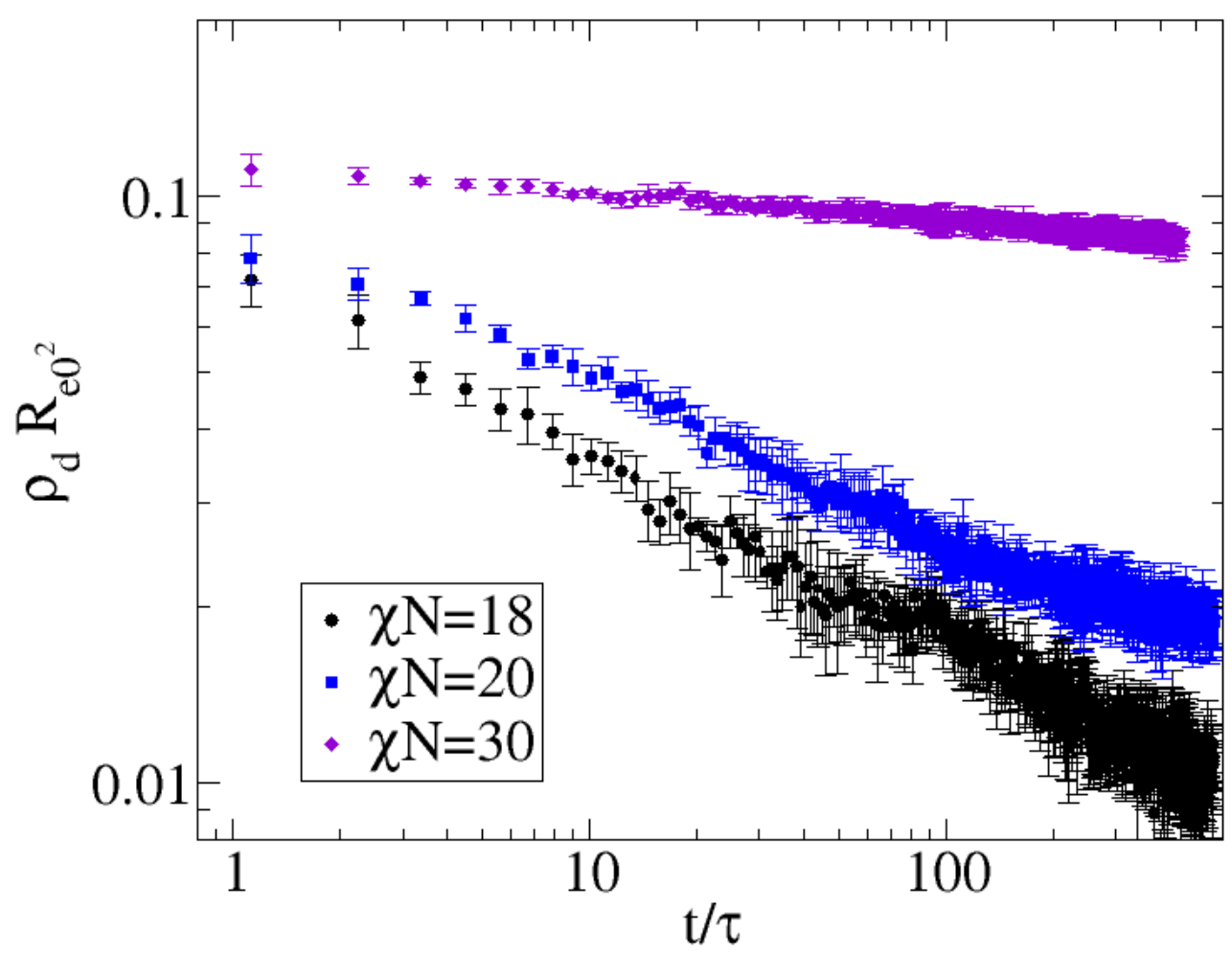

FIGURE 4.16: The density of defects is plotted as a function of simulation time $t$ with a system size $L=40 R_{\mathrm{e} 0}$ quenched to $\chi N=18,20$ and 30. Results are averaged over five independent simulations.

the defect density rapidly decreases and at $t=8 \tau$ these large systems contain less than 10 defects of this type. At high incompatibility, however, the decrease of the number of these defects is significantly slower, i.e., if we postulated in a power law the concomitant exponent $\eta_{\mathrm{d}}^{\text {id }} \approx 0.01$ would be very small. The protracted behavior at large incompatibility can be rationalized by the singlechain dynamics that is involved in the annihilation of isolated domains. In order to eliminate an isolated domain by shrinkage, blocks of the encircled, isolated domain have to diffuse through a domain of the opposite species. This imparts a large free-energy barrier on the single-chain motionbecasue the lbock is exposed to a hostile environment, resulting in an exponential slowing down with $\chi N$ for this intrinsically slow process.

The stability of the defects is determined by the minimal free-energy barrier to eliminate the defect. By means of the string method, we discuss the defect annihilation process on the view of the free-energy landscape in the next section. 


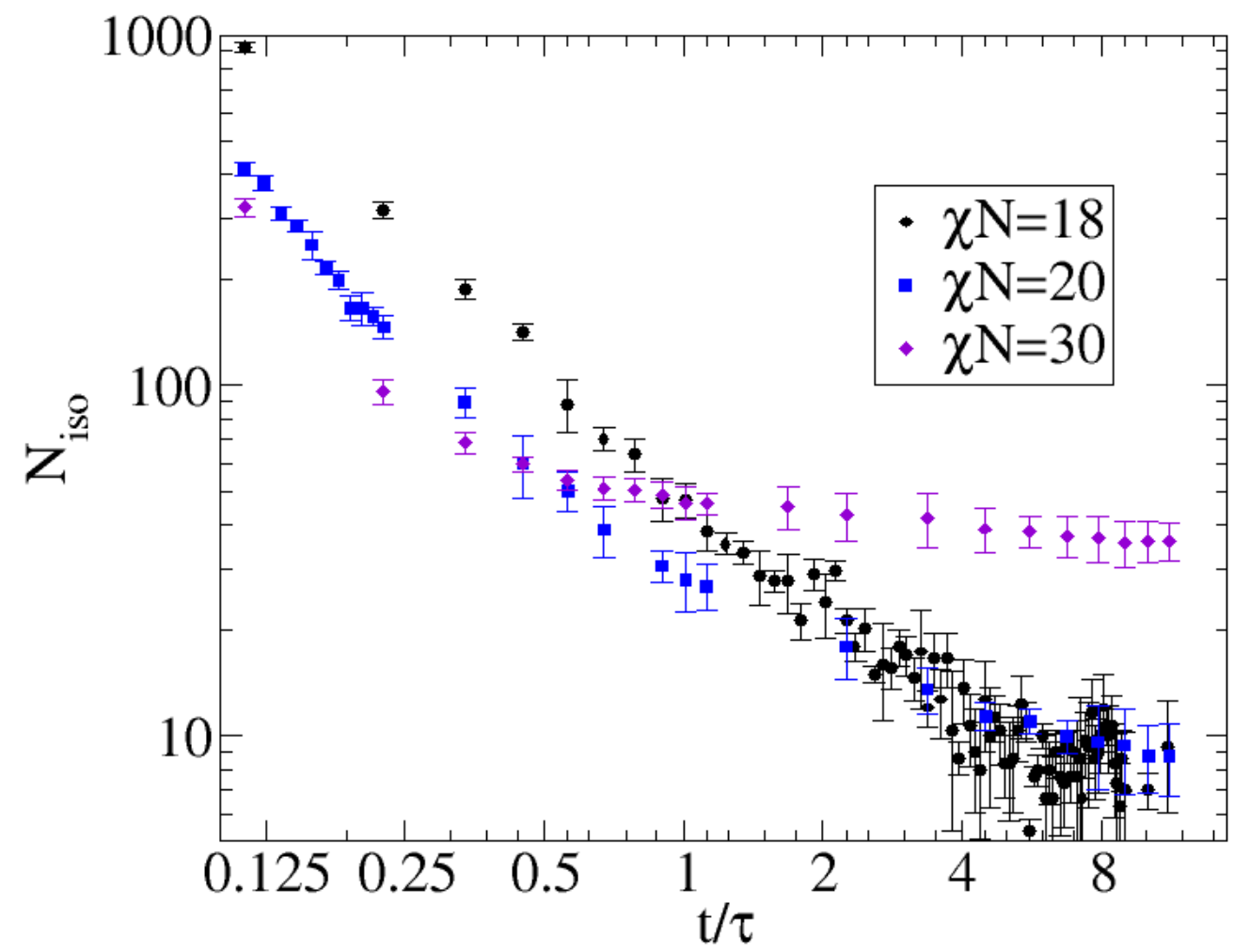

FIGURE 4.17: The number of isolated block domains estimated by quenching $\chi N=18,20$, and 30 is plotted as a function of simulation time $t(\tau)$. The results are averaged by 5 independent simulations.

\subsubsection{Minimum free-energy path of two typical defects}

In this subsection, we will illustrate two typical defect-annihilation mechanisms using two-dimensional SCFT calculations and particle-based simulations in a thin film. The transition states may involve non-trivial three-dimensional structures in the particle-based simulations [130]. The free-energy barrier obtained by the string method in conjunction with two-dimensional SCFT calculations is expected to overestimate the barriers but still provide qualitative insights into the free-energy landscape [126].

\subsubsection{Bifurcation in the free-energy landscape}

In this section, we investigate an example of defect annihilation, which is observed by a particle-based simulation after a quench from the disordered state to $\chi N=30$. The transition process is depicted in Fig. 4.18. The system size is $L=8 R_{\mathrm{e} 0}$ and, unlike previous simulations, we use a smaller invariant degree 

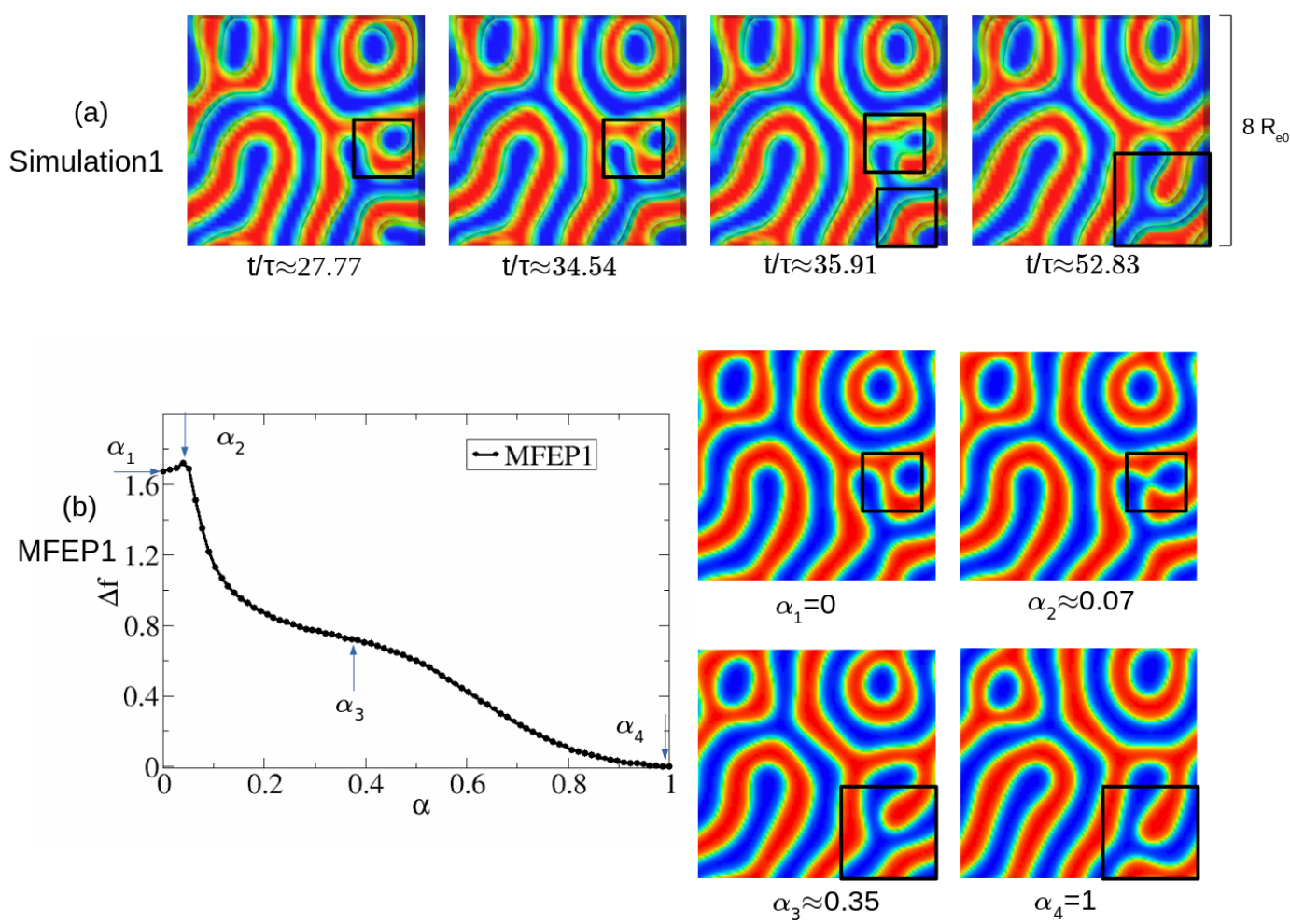

FIGURE 4.18: (a) The breaking of a domain is depicted by a sequence of snapshot (marked by the black square), in the course of a quench from the disordered phase to $\chi N=30$. The invariant degree of polymerization is reduced to $\overline{\mathcal{N}}=70^{2}$, in the SCMF simulations. (b) A MFEP is obtained by initializing the string with morphologies before and after the change of domain morphology. The left panel shows the free-energy profile $\Delta f$ in units of $k_{\mathrm{B}} T$ per chain, whereas the right panel depicts morphologies along the MFEP.

of polymerization, $\sqrt{\overline{\mathcal{N}}}=70$, to facilitate thermally activated processes in the simulation.

The sequence of snapshots are shown in Fig. 4.18 (a). One domain breaks at around $t \approx 41 \tau$, in turn, creates an end of a lamella (dislocation). We initialize the string calculation by uniformly interpolating between the two morphologies obtained before and after the breaking of the domain in the particle-based simulations. We do not constrain the morphologies at the two ends of the string. As a result, they will converge towards metastable states according to AllenCahn dynamics. Two inherent morphologies before and after the change of the domain topology are identified, i.e., the end points of the string. The calculation of the MFEP1 provides the transition path between the two inherent morphologies. The free-energy profile and snapshots along the MFEP1 are shown in panel (b) of Fig. 4.18. 
We find that there is a small free-energy barrier along the MFEP, which is related to the change of domain topology. At $\alpha \approx 0.31$, the free-energy profile exhibits a shoulder, which indicates that the thermodynamic driving force for morphological changes is rather small. The snapshot along the MFEP1 reveal that this feature is associated with the expansion and re-orientation of the created end of the lamella. In the Fig. 4.18 (b) bottom right, we highlight this process by the square in the two last morphologies. In the simulation we expect that such a process, approaching the inherent morphology after the breaking of the connection, is rather protracted.

The free-energy barrier of the topological change at $\alpha \approx 0.05$ is rather small. For $\sqrt{\overline{\mathcal{N}}}=70, L=8 R_{\mathrm{e} 0}$, and $D=R_{\mathrm{e} 0}$, the free-energy barrier, $\Delta f_{\mathrm{b}} \approx 0.0007 k_{\mathrm{B}} T$ per chain corresponds to a small barrier of about $3.1 k_{\mathrm{B}} T$. The value of the freeenergy barrier is not significantly larger than the thermal energy scale, $k_{\mathrm{B}} T$. It is expected that the system can escape the inherent morphology within a short simulation time. We verified this by employing the inherent morphology at $\alpha=0$ as an initial density distribution for particle-based simulations. For 10 independent simulations, we observe that all simulations escape from this inherent morphology within $\Delta t=10 \tau$.

We observe that some runs, starting from the inherent morphology $\alpha=0$ of MFEP1, do not follow the MFEP1. In the particle-based simulations, we have observed an alternate transition path, as shown in Fig. 4.19 (a). ${ }^{1}$ After $\Delta t=$ 0 and $6.71 \tau$, the domain morphology changes in accord with MFEP1. Later, instead of relaxing towards the inherent morphology, $\alpha=1$, of MFEP1 via the protracted, expansion and re-orientation of the created end of the lamella, we find that another isolated domain breaks open in the top right corner of the system (cf. zoom-in in Fig. 4.19 (a)) around $\Delta t=7.87 \tau$, i.e., rather rapidly after the first change of domain topology. This new event gives rise to further changes of the domain morphology (see simulation snapshot at $\Delta t=21.37 \tau$ ).

In order to understand the transition of this second change of domain topology between $\Delta t=6.71 \tau$ and $7.87 \tau$, we investigate the free-energy landscape by the MFEP2 between the inherent morphology that corresponds to the configuration at $\Delta t=6.71 \tau$, i.e., the end of MFEP1, and the inherent morphology that corresponds to the morphology at $\Delta t=7.87 \tau$. We find that the inherent morphology of the simulation snapshot at $\Delta t=7.87 \tau$ has the same domain topology as the

\footnotetext{
${ }^{1}$ If we increase $\overline{\mathcal{N}}$ to $128^{2}$, all runs would follow the MFEP1.
} 
(a)

Simulation2

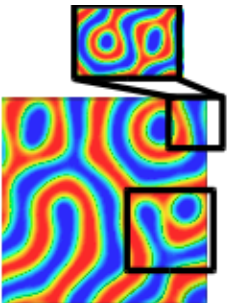

$\Delta \mathrm{t} / \mathrm{t}=0$

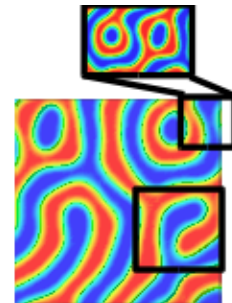

$\Delta \mathrm{t} / \mathrm{t} \approx 6.71$

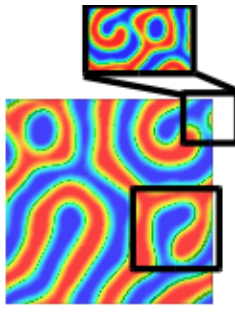

$\Delta \mathrm{t} / \mathrm{t} \approx 7.87$

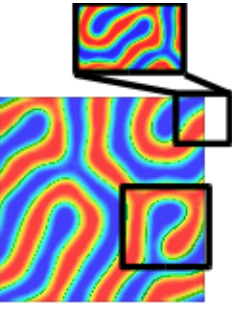

$\Delta \mathrm{t} / \mathrm{\tau} \approx 21.37$

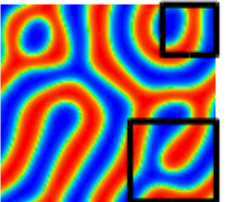
$\alpha_{1}^{\prime}=0$

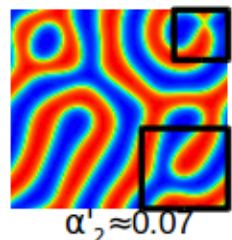

(b)
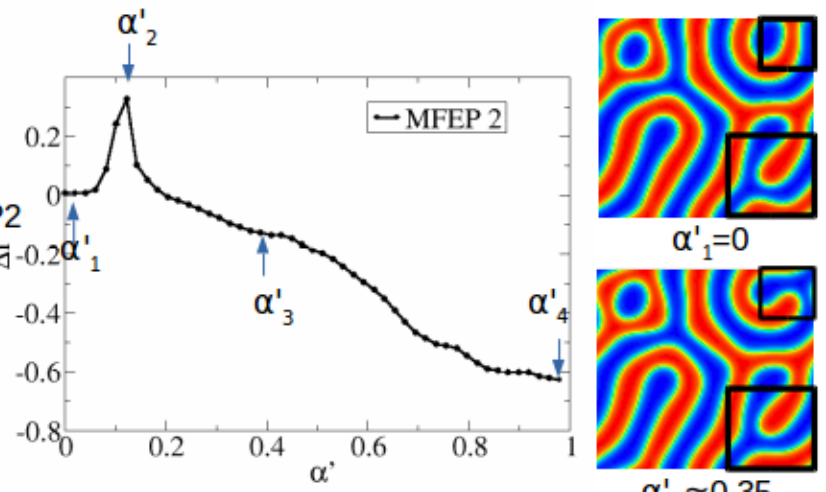

$\alpha^{\prime} \approx 0.35$

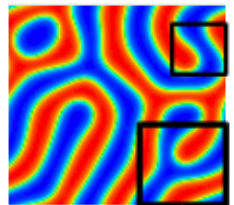

$\alpha_{4}^{\prime}=1$

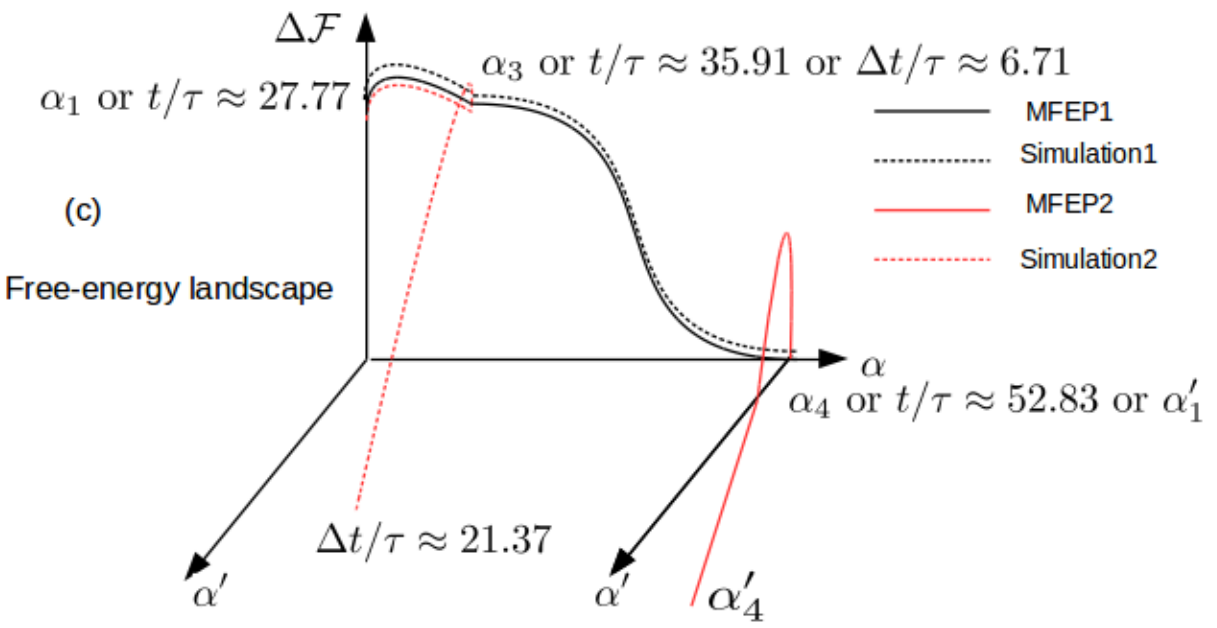

FIGURE 4.19: (a) Snapshots of a defect annihilation process which is similar to a B-core dislocation dipole defect. The particle-based simulation is initialized with the morphology $\alpha=0$ in MFEP1. The snapshot of the B-core dislocation dipole defect is enlarged in the inset. (b) Snapshots and free-energy profile of the MFEP2, in which the starting morphology $\alpha^{\prime}=0$ is the ending morphology in MFEP1 $\alpha=1$ (c) Schematic representation of the free-energy landscape associated with MFEP1, MFEP2, simulation1, and simulation2. 
simulation snapshot. The simulation 2 from $\Delta t=0$ to $\Delta t=7.87 \tau$, corresponds to a process that concatenates MFEP1 and MFEP2.

In Fig. 4.19 (b), we present the free-energy profile of MFEP2 and the corresponding morphologies. We find that the change of domain topology along the MFEP2 involves a significant free-energy barrier, corresponding to approximately $22.4 k_{\mathrm{B}} T$ in the particle-based simulations. After overcoming the very shallow barrier of MFEP1, we find that the particle-based simulation approaches to the inherent structure $\alpha=1$ of MFEP1 (or equivalently $\alpha^{\prime}=0$ of MFEP2) remained there for more than $750 \tau$.

To make it easier to understand, the relation between the two MFEPs and the alternate evolution in simulation 2 is sketched in panel (c) of Fig. 4.19. We conclude that the strain-field mediated, co-operative rearrangements of the morphology that occur between the barrier and the end point of MFEP1 (starting point of MFEP2) do significantly change the free energy, even if they have the same domain topology. The domain morphology evolves in response to the change of the strain-field mediated interactions, immediately, after the first topological change. In the simulation2, before the system has approached the corresponding inherent morphology, a second topological change occurs. This suggests that the barrier is only of the order $k_{\mathrm{B}} T$. The barrier for a second change of domain topology significantly increases, in the course of the relaxation towards the corresponding inherent morphology. When the system reached the inherent morphology, quantified by the barrier along the MFEP2, the second topological change would incur the much larger free-energy barrier. As a result, the barriers for changes of the domain topology, i.e., the barrier can be smaller than suggested by the MFEP between inherent morphologies can be significantly altered due to strain-field mediated interactions.

\subsubsection{Trifurcation in the free-energy landscape}

In another independent simulation, we investigate a process to eliminate an isolated block defect. The system size is also set as $L=8 R_{\mathrm{e} 0}$ and the FloryHuggins parameter is $\chi N=30$. The invariant degree of polymerization is set to $\sqrt{\overline{\mathcal{N}}}=70$. 


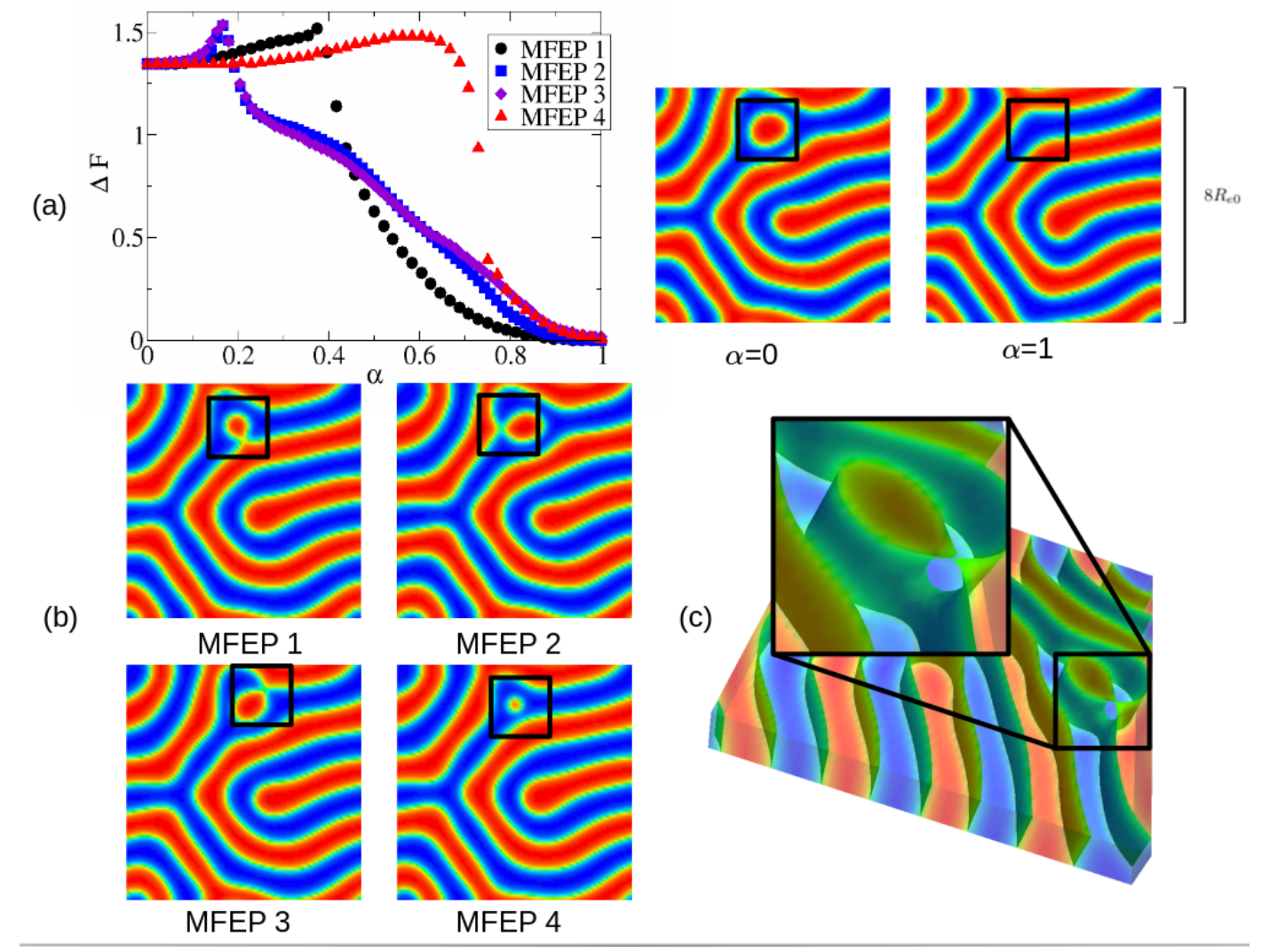

FIGURE 4.20: (a) Right: The free-energy of four MFEPs to eliminate an isolated block domain. Left: The starting $\alpha=0$ and ending $\alpha=1$ meta-stable morphologies are obtained by the SCFT. (b) Snapshots of intermediate states in four MFEPs. (c) Scheme illustration of the annihilation process by MFEP3 in the three-dimensional system.

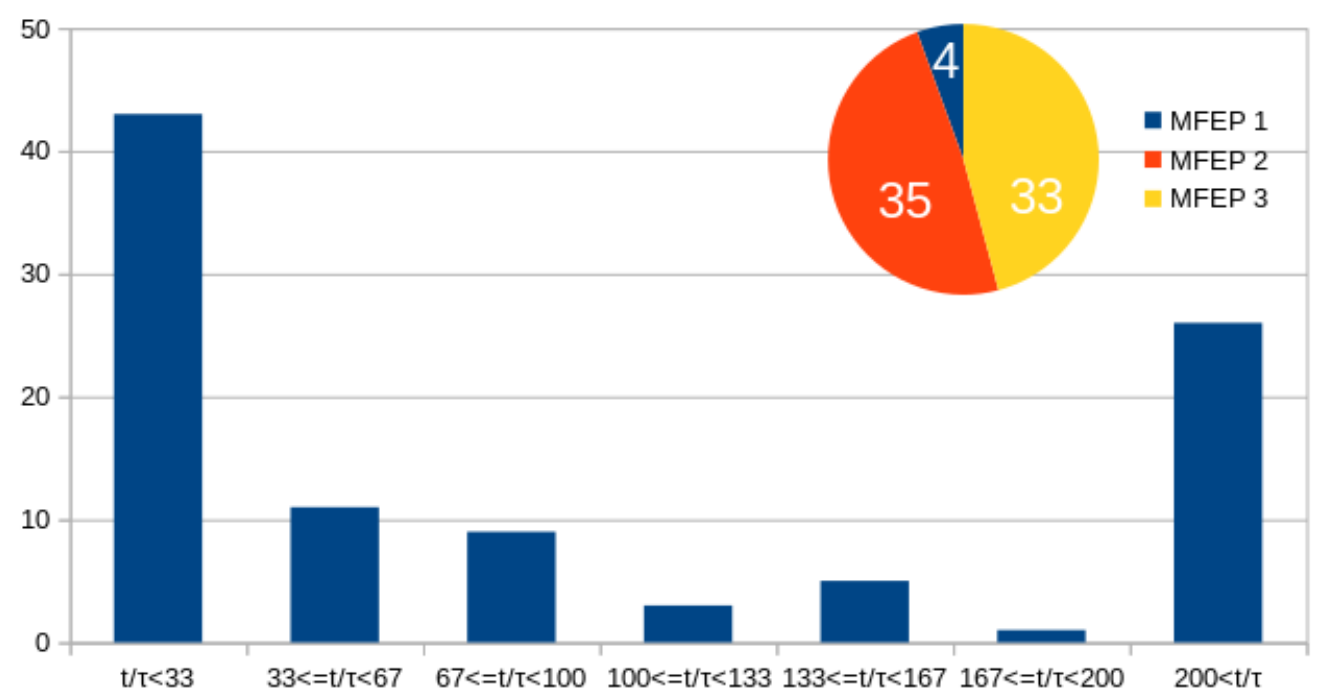

FIGURE 4.21: The statistics of the relaxation time to eliminate the isolated block defect for 100 particle-based simulations with an initial density configuration $\alpha=0$. 
One isolated block defect is eliminated by thermal fluctuations at $t=665 \tau$. We select two morphologies in the simulation at $t=665 \tau$ and $t=669 \tau$. We quenched these two morphologies with the SCFT method, as shown in Fig. 4.20 (a) left.

As shown in Fig. 4.20 (b), there are four possible MFEPs to eliminate the isolated A domain. MFEP1 is merging the isolated block into the lower stripe domain. MFEP2 and MFEP3 are merging into left and right stripe domains, respectively. The fourth MFEP, i.e., the MFEP 4 is an evaporation-like mechanism.

In the simulation, we can estimate the free-energy barrier of four MFEPs. The free-energy barrier of the MFEP1 is about $12.383 k_{\mathrm{B}} T$. The free-energy barrier of the MFEP4 is the lowest which is $9.408 k_{\mathrm{B}} T$. The free-energy barrier of the second and the third MFEP are approximately the same which is about $12.998 k_{\mathrm{B}} T$ due to the symmetry. Above computation is implemented in a two-dimensional lattice, the free-energy barrier is overestimated. We calculate the MFEP3 in a three-dimensional bulk system. As depicted in the Fig. 4.20 (c), in a threedimensional computation, the isolated domain and the line domain get contact with a very thin connection at the initial stage. Later, the thin connection gets thicker in the direction that is perpendicular to the plane of the quasi-twodimensional system. The value of the free-energy barrier on a $3 \mathrm{D}$ calculation is reduced to $11.827 k_{\mathrm{B}} T$ in the simulation.

We run 100 independent simulations which are initialized with the metastable morphology $\alpha=0$. The total simulation time is about $332 \tau$. We make a statistics on these simulations which is shown in Fig. 4.21. There are 28 simulations remaining the same topological structure with the morphology at $s=0$ within a simulation time $332 \tau$. Only 4 simulations eliminate the isolated block domain with the MFEP1. Most simulations chose the MFEP 2 and the MFEP 3 to eliminate the defect. Their numbers are 35 and 33, respectively.

Although the free-energy barrier in the MFEP4 is the smallest. But we do not observe this process in the simulation. This evaporation-like mechanism is difficult to realize for diblock copolymer melts in the strong-segregation regime. Because the Onsager-coefficient that translates the thermodynamic force into the composition flow is proportional to $\left(1-m^{2}\right)$. It is difficult to transport different species through a domain [130]. The MFEP 1 also involves an evaporation-like mechanism in the initial stage. It first shrinks to a smaller block, then it merges 
to the lower line domain. In our simulations, only four samples out of 100 independent simulations follow the MFEP1. 


\section{Chapter 5}

\section{Summary and outlook}

\subsection{Summary}

In this thesis, we investigate the non-equilibrium, structure formation process in block copolymers. We can, on one hand, investigate the structure formation process kinetically by observing mass, large-scale, particle-based simulations, or on the other hand, thermodynamically by exploring the free-energy landscape.

On the perspective of the free-energy landscape, we investigate two important quantities in the chapter 3: one is the thermodynamic force (i.e., the chemical potential), which drives the formation of various long-range order structures. The other one is the Onsager coefficient, which transmits the thermodynamic forces along the molecular backbone.

The thermodynamic force is directly related to the free-energy landscape. We propose a very accurate and computationally efficient method (the umbrella potential method in conjunction with SCFT) to obtain the chemical potential. For the multiple-dimensional, free-energy landscape, it is not possible or necessary to obtain its full knowledge. By means of the string method, we can obtain the most useful information, i.e., the minimum free-energy path. It gives the most probable transition path connecting the two thermal states.

The Onsager coefficient cannot be easily obtained analytically. We provide a numerical scheme to directly measure the Onsager coefficient in the particlebased simulations. Although we only focus on the linear polymer chains with 
a simple Rouse dynamics in this article, this method is rather general and can be easily extended to measure the Onsager coefficient in various polymer architectures and dynamics.

The Onsager coefficient, $\Lambda(\mathbf{q}, \omega)$ is time-dependent, which is associated to the single-chain molecular dynamics in a quasi-homogeneous system. Based on the dynamic equation of densities in Eq. 3.64, we can preliminary estimate the Onsager coefficient for a long-time limit. In the sub-diffusive regime, the Onsager coefficient is time-dependent.

We also investigate the structure formation process from the kinetic perspective. By employing massive particle-based simulations, we investigate the kinetics of pattern formation in quasi-two-dimensional symmetric diblock copolymer melts. When lamellar-forming block copolymer melts are quenched far below the order-disorder transition temperature, the quasi-two-dimensional systems form an isotropic morphology with finite-size lamellar grains. In the structure ordering process, these grains will grow up by the grain-coarsening process. From the observations of morphologies in particle-based simulations, domains of A block or B block will break and connect at the boundary of lamellar grains due to the frustrated orientation of lamellar grains.

The growth of the lamellar grains dramatically slows down when the temperature decreases. This phenomenon is confirmed by measuring the correlation length. From $\chi N=16$ to $\chi N=20$, the relaxation time of the structure ordering increases dramatically with $\chi N$. The slow ordering process in block copolymers has been liken to the glassing-forming systems, which means that the free-energy landscape is rugged below the critical temperature. The basins in the free-energy landscape are related to the topology of the morphologies. By means of SCFT, we quench the morphologies obtained in the simulation to the inherent morphologies. We find that morphologies which have an identical topology are in the same free-energy basins. The breaking or connecting domains which changes the topology of the morphology is usually a rare event of basin-hopping. We use the Euler characteristic, $\chi_{\mathrm{E}}$ to monitor the change of the topology. The topology of the morphology is altered via eliminating unstable structures, i.e., defects. We classify three kinds of defects according to their topological structure. The number of defects in the morphology decays with a power-law, $t^{-\eta_{D} t}$. The decay exponent $\eta_{D}$ decreases with $\chi N$. This suggests that the number of basins in the free-energy landscape increases with $\chi N$. 
In the end, we investigate the mechanism to eliminate unstable structures via the string method in conjunction with SCFT. The possibility to escape the freeenergy basin is mainly determined by the value of the free-energy barrier along the free-energy profile.

\subsection{Outlook}

In this thesis, we put a lot of efforts to investigate the structure formation process in block copolymers. We try to cover as many topics as we can, but there are still many open questions which worth further investigations.

(i) To explore the free-energy landscape, we only consider slow variables (e.g., the composition of diblock copolymers) as the order parameter and neglect the influence of the dynamics of the molecular chain. In fact, this effect is also important, especially when the polymer chain is strongly stretched.

The major difficulty to take into account the dynamics of a polymer chain is to determine a proper order parameter. One possible strategy to capture the polymer chain conformation is to utilize the Rouse mode as the order parameter [57].

(ii) The string method has become a very mature technique to obtain the MFEP. One has a good understanding of various defect annihilation mechanism via the MFEP. One question still remains untouched is that how the structure formation processes are related to the molecular architecture. This is a very complex question and we will only investigate a defect removal process in triblock copolymers in Sec. 3.5.3. We compare the B-core dislocation dipole defect in symmetric diblock copolymers with the triblock copolymers. As expected, we find that molecular architectures have an impact on the defect annihilation process. We still do not understand the mechanism and this question is to be understood by a further investigation. 


\section{Appendix A}

\section{Other umbrella potentials}

In the section 3.2.2 and 3.2.3, we introduce the exact constraint method and the umbrella potential method to derive the free energy, $\mathcal{F}_{\text {target }}[m]$ and the chemical potential, $\mu[\mathrm{m}]$ for a specific configuration characterized by the order parameter, $m$.

For block copolymer melts, we use the constraint, $\delta\left(m-\phi_{A}+\phi_{B}\right)$. In many circumstances, the choice of the constraint is not unique. For example, in an incompressible diblock copolymer melt, we can also use a constraint, $\delta\left(\phi_{A}-\right.$ $\left.\tilde{\Phi}_{A}\right)$, where $\tilde{\Phi}_{A} \equiv(1+m) / 2$. These two constraint are equivalent.

To use the new constraint, $\delta\left(\phi_{A}-\tilde{\Phi}_{A}\right)$, the corresponding free-energy functional, $\mathcal{F}_{\text {target }}\left[\tilde{\Phi}_{A}\right]$ is modified as,

$$
\begin{aligned}
\frac{\mathcal{F}_{\text {target }}\left[\tilde{\Phi}_{A}\right]}{n k_{\mathrm{B}} T}= & -\ln \mathcal{Q}+\frac{1}{V} \int \mathrm{d} \vec{r}-\Xi\left(1-\Phi_{A}-\Phi_{B}\right) \\
& +\chi N \Phi_{A} \Phi_{B}-W_{A} \Phi_{A}-W_{B} \Phi_{B} \\
& +W_{\text {exphi }}\left(\tilde{\Phi}_{A}-\Phi_{A}\right) .
\end{aligned}
$$

The self-consistent equations are modified as, 


$$
\begin{aligned}
\Xi & =\left(W_{A}+W_{B}-\chi N+W_{e x}\right) / 2 \\
W_{e x} & =-\chi N\left(2 \tilde{\Phi}_{A}-1\right)-\left(W_{A}-W_{B}\right) \\
W_{A} & =\Xi+\chi N\left(1-\tilde{\Phi}_{A}\right)-W_{\text {exphi }} \\
W_{B} & =\Xi+\chi N \tilde{\Phi}_{A}
\end{aligned}
$$

The chemical potential $\mu[m]$ is newly derived as,

$$
\frac{\mu[m]}{k_{\mathrm{B}} T}=\frac{1}{\sqrt{\mathcal{N}} k_{\mathrm{B}} T} \frac{\delta \mathcal{F}}{\delta m}=\frac{1}{2} \frac{\delta \mathcal{F}}{\delta \tilde{\Phi}_{A}}=\frac{1}{2} W_{\text {exphi }}
$$

Similarly, instead of using the umbrella potential in equation 3.24, we can use the chemical potential $\mathcal{H}_{\lambda 1}$ or $\mathcal{H}_{\lambda 2}$

$$
\begin{aligned}
\frac{\mathcal{H}_{\lambda 1}}{\sqrt{\overline{\mathcal{N}}} k_{\mathrm{B}} T} \equiv & \frac{\lambda N}{2 R_{e 0}^{3}} \int \mathrm{d} \vec{r}\left(\tilde{\Phi}_{A}-\hat{\phi}_{A}\right)^{2} \\
& +\frac{\lambda N}{2 R_{e 0}^{3}} \int \mathrm{d} \vec{r}\left(1-\tilde{\Phi}_{A}-\hat{\phi}_{B}\right)^{2} \\
\frac{\mathcal{H}_{\lambda 2}}{\sqrt{\overline{\mathcal{N}}} k_{\mathrm{B}} T} \equiv & \frac{\lambda N}{2 R_{e 0}^{3}} \int \mathrm{d} \vec{r}\left(\tilde{\Phi}_{A}-\hat{\phi}_{A}\right)^{2} .
\end{aligned}
$$

The self-consistent equations for $\mathcal{H}_{\lambda 1}$ is,

$$
\begin{aligned}
\Xi & =\left(W_{A}+W_{B}-\chi N\right) / 2 \\
W_{A} & =\Xi+\chi N\left(1-\tilde{\Phi}_{A}\right)-\lambda N\left(\tilde{\Phi}_{A}-\Phi_{A}\right) \\
W_{B} & =\Xi+\chi N \tilde{\Phi}_{A}-\lambda N\left(1-\tilde{\Phi}_{A}-\Phi_{B}\right)
\end{aligned}
$$

The self-consistent equations for $\mathcal{H}_{\lambda 2}$ is,

$$
\begin{aligned}
\Xi & =\left[W_{A}+W_{B}-\chi N+\lambda N\left(\Phi_{A}-\tilde{\Phi}_{A}\right)\right] / 2 \\
W_{A} & =\Xi+\chi N\left(1-\tilde{\Phi}_{A}\right)-\lambda N\left(\tilde{\Phi}_{A}-\Phi_{A}\right) \\
W_{B} & =\Xi+\chi N \tilde{\Phi}_{A}
\end{aligned}
$$


Importantly, the chemical potential $\mu_{\lambda 1}$ and $\mu_{\lambda 2}$ is,

$$
\begin{aligned}
& \frac{1}{\sqrt{\overline{\mathcal{N}}}} \frac{\mu_{\lambda 1}\left[\tilde{\Phi}_{A}\right]}{k_{\mathrm{B}} T}=\frac{1}{k_{\mathrm{B}} T \sqrt{\overline{\mathcal{N}}}} \frac{\delta \mathcal{F}_{\lambda 1}}{\delta \tilde{\Phi}_{A}}=\lambda N\left(2 \tilde{\Phi}_{A}-1-\Phi_{A}+\Phi_{B}\right) \\
& \frac{1}{\sqrt{\overline{\mathcal{N}}}} \frac{\mu_{\lambda 2}\left[\tilde{\Phi}_{A}\right]}{k_{\mathrm{B}} T}=\frac{1}{k_{\mathrm{B}} T \sqrt{\overline{\mathcal{N}}}} \frac{\delta \mathcal{F}_{\lambda 2}}{\delta \tilde{\Phi}_{A}}=\lambda N\left(\tilde{\Phi}_{A}-1-\Phi_{A}\right)
\end{aligned}
$$

We have the following relation,

$$
\mu[m]=\frac{1}{2} \mu_{\lambda 1}\left[\tilde{\Phi}_{A}\right]=\frac{1}{2} \mu_{\lambda 2}\left[\tilde{\Phi}_{A}\right]
$$




\section{Appendix B}

\section{Random-phase approximation}

In the following, we will employ the random-phase approximation to derive the structure factor for symmetric diblock copolymers.

The structure factors are important quantities for both experimentalists and theorists. Many experimental techniques such as the elastic radiation scattering experiments (light, X-Ray, or neutron scattering) can directly measure the structure factor of block copolymers according to the linear response theorem [19]. Theoretically, these functions can be used to accelerate the computation of SCFT equations [92]. In the section 3.3.1, we also show that the Onsager coefficient is nonlocal, which is described by the single-chain correlation function [119].

Before embarking on the multiple-component block copolymer system, we will first calculate the structure factor for ideal chains as a preparation. We consider a linear polymer chain which is confined in a volume $V=L \times L \times L$ with a periodical boundary condition. The number of segments along the single chain is $N$. The coordinate of the $s^{t h}$ monomer is denoted as $\vec{r}(s)$. The local density of the single chain is represented as,

$$
\hat{\phi}(\vec{r})=\frac{1}{\rho} \sum_{s=0}^{N} \delta(\vec{r}-\vec{r}(s))
$$

where $\rho$ equals to $N / V$.

The single-chain correlation function, $g_{0}\left(\vec{r}, r^{\prime}\right)$ is defined as,

$$
g_{0}\left(\vec{r}, r^{\prime}\right) \equiv\left\langle\hat{\phi}(\vec{r}) \hat{\phi}\left(\vec{r}^{\prime}\right)\right\rangle_{0}
$$


The thermodynamic average $\langle\ldots\rangle_{0}$ denotes the canonical ensemble average which only subjected to the bonded interactions in Eq. 2.5. The structure factor of an ideal chain is the Fourier transform the single-chain correlation function,

$$
S_{0}\left(\mathbf{q}, \mathbf{q}^{\prime}\right) \equiv \int \mathrm{d} \vec{r} \mathrm{~d} \vec{r}^{\prime} g_{0}\left(\vec{r}, r^{\prime}\right) \exp \left\{i \vec{r} \mathbf{q}+i \vec{r}^{\prime} \mathbf{q}^{\prime}\right\}
$$

The $S_{0}\left(\mathbf{q}, \mathbf{q}^{\prime}\right)$ can be directly calculated as,

$$
\begin{aligned}
S_{0}\left(\mathbf{q}, \mathbf{q}^{\prime}\right) & =\frac{N^{2}}{\rho_{0}^{2}} \int \mathrm{d} \vec{r} \int \mathrm{d} \vec{r}^{\prime} e^{-i \mathbf{q} \vec{r}-i \mathbf{q}^{\prime} \vec{r}^{\prime}}\left\langle\phi(\vec{r}) \phi\left(\vec{r}^{\prime}\right)\right\rangle_{0} \\
& =\frac{N^{2}}{\rho^{2}} \int_{s=0}^{1} \mathrm{~d} s \int_{s^{\prime}=0}^{1} \mathrm{~d} s^{\prime} \int \mathrm{d} \vec{r} \int \mathrm{d} \vec{r}^{\prime} e^{-i \mathbf{q} \vec{r}-i \mathbf{q}^{\prime} \vec{r}^{\prime}}\left\langle\delta(\vec{r}-\vec{r}(s)) \delta\left(\overrightarrow{r^{\prime}}-\vec{r}\left(s^{\prime}\right)\right)\right\rangle_{0} \\
& =\frac{N^{2}}{\rho^{2}} \int_{s_{1}=0}^{1} \int_{s_{2}=0}^{1} \mathrm{~d} s_{1} \mathrm{~d} s_{2} e^{-\frac{R_{e 0}^{2}}{6} \mathbf{q}^{2}\left|s_{2}-s_{1}\right|} \delta\left(\mathbf{q}+\mathbf{q}^{\prime}\right) \frac{1}{V} .
\end{aligned}
$$

We use $S_{0}(\mathbf{q}) \equiv \int \mathrm{d} \mathbf{q}^{\prime} S\left(\mathbf{q}, \mathbf{q}^{\prime}\right)$, and make the substitution of $x \equiv \frac{R_{\mathrm{e}}^{2}}{6} \mathbf{q}^{2}$,

$$
S_{0}(\mathbf{q})=N g(x)=\frac{2 N}{x^{2}}\left(x-1+e^{-x}\right),
$$

where $g$ is the Debye function.

The limiting behavior of the structure factor $S(\mathbf{q})$ is simplified as,

$$
S(\mathbf{q})= \begin{cases}N\left(1-\mathbf{q}^{2} R_{e 0}^{2} / 18\right) & , \mathbf{q} R_{\mathrm{e} 0} \ll 1 \\ 12 N / \mathbf{q}^{2} R_{e 0}^{2} & 1 \ll \mathbf{q} R_{\mathrm{e} 0}\end{cases}
$$

By the inverse Fourier transform on $S(\mathbf{q})$, we can obtain the single-chain correlation function $g_{0}\left(\vec{r}, r^{\prime}\right)$. The single-chain correlation function, $g_{0}\left(\vec{r}, r^{\prime}\right)$ is isotropic in the space. $g_{0}\left(\vec{r}, r^{\prime}\right)$ can be represented as a function of the two points distance $r=|\vec{r}-\vec{r}|$ with the following equation,

$$
g_{0}(r)=\frac{1}{4 \pi r^{2}} \int_{\left|\vec{r}-\overrightarrow{r^{\prime}}\right|=r} \mathrm{~d} \vec{r} \mathrm{~d} \vec{r}^{\prime} g_{0}\left(\vec{r}, \vec{r}^{\prime}\right) .
$$

We have the normalization relation that,

$$
4 \pi \int \mathrm{d} r r^{2} g_{0}(r)=V^{2}
$$


In the limit of $q \ll 1, S(\mathbf{q}) \sim 1 / \mathbf{q}^{2}$. The inverse Fourier transform of $1 / \mathbf{q}^{2}$ is $1 / r$. The second term in Eq. B.10 can be neglected, which gives,

$$
V^{2} g_{0}(r) \approx \frac{3 N}{\pi R_{e 0}^{3} r} .
$$

At another limit $1 \ll q, S(\mathbf{q}) \sim \exp \{-\mathbf{q}\}$. The single-chain, correlation function exponentially decays with the distance $r$.

The analytical form of the single-chain correlation function, $g_{0}(r)$ is,

$$
V^{2} g_{0}(r)=\frac{3 N}{\pi R_{\mathrm{e} 0}^{2} r}\left[1-2 \int_{0}^{1} \mathrm{~d} \epsilon \operatorname{erf}\left(\sqrt{\frac{3 r}{2 \epsilon N} \bar{b}}\right)\right] .
$$

The physical meaning of the single-chain correlation function $g_{0}(r)$ is that we randomly select a monomer on the chain and measure the probability to find another monomer at a distance $r$.

To numerically verify the behavior of the single-chain correlation function, we simulate an ideal chain in a cubic box with $L=4 R_{\mathrm{e} 0}$ in all direction to obtain $g_{0}(r)$. The mean-square end-to-end distance $R_{e}^{2}=R_{\mathrm{e} 0}^{2}$. The polymer chain can fully span in the box, such that the finite size effect is negligible. The discretization of the polymer chain is $N=32$.

As is shown in the Fig. B.1, the single-chain correlation function $\rho^{2} g_{0}(r)$ exponentially decay with the distance $r$ at large $r$ limit.

Next we embark on the multi-chain system. We calculate the structure factor of diblock copolymer melts by the RPA. The fraction of A block is denoted as $f_{A}$ and the fraction of $\mathrm{B}$ block is $f_{B}=1-f_{A}$.

The basic idea of the RPA approach is to replace interactions between polymers by an effective external fields. Thus the partition function of a multiple chain system is decoupled and can be written as a summation of single-chain partition functions $\mathcal{Q}\left[W_{A}, W_{B}\right]$ under weak external fields $W_{A}$ and $W_{B}$.

So we first consider the single-chain partition function $\mathcal{Q}\left[W_{A}, W_{B}\right]$ which can be expanded in terms of the external potentials, 


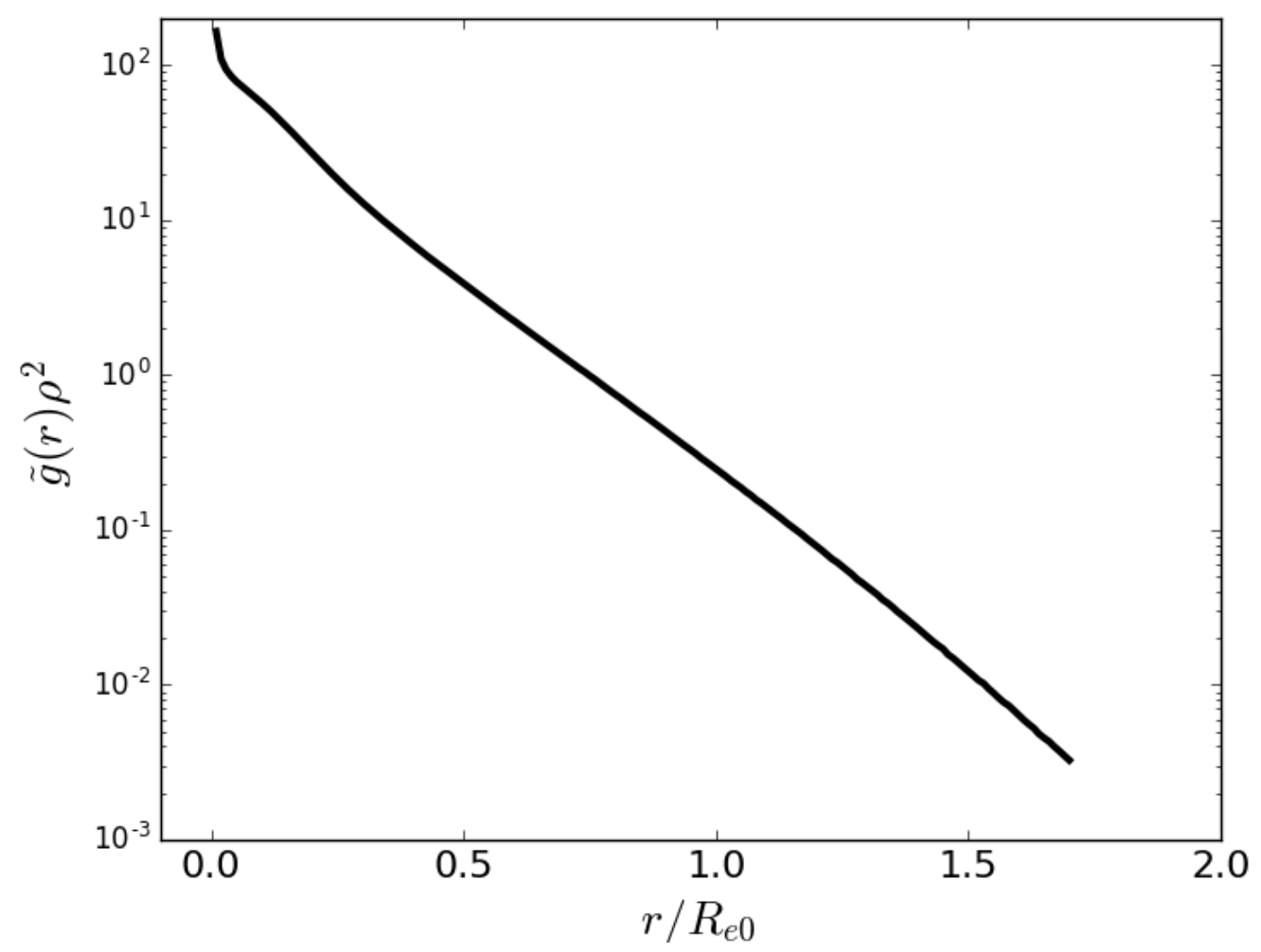

FIGURE B.1: The single-chain correlation function is plotted as a function of the distance $r$.

$$
\begin{aligned}
\frac{\mathcal{Q}}{\mathcal{Q}_{0}} \equiv & \left\langle\exp \left\{-\int \mathrm{d} \vec{r} \frac{1}{V}\left(\phi_{A 1} W_{A}+\phi_{B 1} W_{B}\right)\right\}\right\rangle_{0} \\
= & \left\langle\exp \left\{-\int \mathrm{d} \mathbf{q} \frac{1}{V}\left(\phi_{A 1}(\mathbf{q}) W_{A}(-\mathbf{q})+\phi_{B 1}(\mathbf{q}) W_{B}(-\mathbf{q})\right)\right\}\right\rangle_{0} \\
= & 1-\left\langle\int \mathrm{d} \mathbf{q} \frac{1}{V}\left(\phi_{A 1}(\mathbf{q}) W_{A}(-\mathbf{q})+\phi_{B 1}(\mathbf{q}) W_{B}(-\mathbf{q})\right)\right\rangle_{0} \\
+ & \frac{1}{2}<\int \mathrm{d} \mathbf{q} \mathrm{d} \mathbf{q}^{\prime} \frac{1}{V^{2}}\left\{\phi_{A 1}(\mathbf{q}) W_{A}(-\mathbf{q})+\phi_{B 1}(\mathbf{q}) W_{B}(-\mathbf{q})\right\} \\
& \left\{\phi_{A 1}\left(\mathbf{q}^{\prime}\right) W_{A}\left(-\mathbf{q}^{\prime}\right)+\phi_{B 1}\left(\mathbf{q}^{\prime}\right) W_{B}\left(-\mathbf{q}^{\prime}\right)\right\}>_{0}+o\left(W_{A, B}^{2}\right)
\end{aligned}
$$

where the partition function of an ideal chain is,

$$
\mathcal{Q}_{0}=V\left(\sqrt{\frac{2 \pi R_{e 0}^{2}}{3 N}}\right)
$$


$\phi_{A 1}$ and $\phi_{A 1}$ are the single-chain density of A and B species, respectively. They are defined as,

$$
\begin{aligned}
& \phi_{A 1}(\vec{r})=\frac{1}{\rho} \sum_{s=0}^{f_{A} N} \delta(\vec{r}-\vec{r}(s)) \\
& \phi_{B 1}(\vec{r})=\frac{1}{\rho} \sum_{s=f_{A} N}^{N} \delta(\vec{r}-\vec{r}(s))
\end{aligned}
$$

The structure factor of AA density and BB density and $\mathrm{AB}$ density is given as,

$$
\begin{aligned}
S_{A A}(\mathbf{q}) & \equiv\left\langle\phi_{A 1}(\mathbf{q}) \phi_{A 1}\left(\mathbf{q}^{\prime}\right)\right\rangle_{0} \\
& =\frac{N^{2}}{\rho^{2}} \int_{s_{1}=0}^{s_{1}=f_{A}} \int_{s_{2}=0}^{s_{2}=f_{A}} \mathrm{~d} s_{1} \mathrm{~d} s_{2} \exp \left\{\frac{R_{\mathrm{e} 0}^{2}}{6} \mathbf{q}^{2}\left|s_{2}-s_{1}\right|\right\} \\
& =N g(f, x) \\
S_{B B}(\mathbf{q}) & \equiv\left\langle\hat{\phi}_{B 1}(\mathbf{q}) \hat{\phi}_{B 1}\left(\mathbf{q}^{\prime}\right)\right\rangle_{0} \\
& =\frac{N^{2}}{\rho^{2}} \int_{s_{1}=f_{A}}^{s_{1}=1} \int_{s_{2}=f_{A}}^{s_{2}=1} \mathrm{~d} s_{1} \mathrm{~d} s_{2} \exp \left\{\frac{R_{e 0}^{2}}{6} \mathbf{q}^{2}\left|s_{2}-s_{1}\right|\right\} \\
& =N g(1-f, x) \\
S_{A B}(\mathbf{q}) & \equiv\left\langle\hat{\phi}_{A 1}(\mathbf{q}) \hat{\phi}_{B 1}\left(\mathbf{q}^{\prime}\right)\right\rangle_{0} \\
& =\frac{N^{2}}{\rho^{2}} \int_{s_{1}=0}^{s_{1}=f_{A}} \int_{s_{2}=f_{A}}^{s_{2}=1} \mathrm{~d} s_{1} \mathrm{~d} s_{2} \exp \left\{\frac{R_{\mathrm{e} 0}^{2}}{6} \mathbf{q}^{2}\left|s_{2}-s_{1}\right|\right\} \\
& =\frac{N}{2}[g(1, x)-g(f, x)-g(1-f, x)]
\end{aligned}
$$

According to the random-phase approximation, we neglect high order terms $o\left(W_{A, B}^{2}\right)$. The first order term is zero, because we employ a periodic boundary condition. The partition function is simplified as,

$$
\frac{\mathcal{Q}}{\mathcal{Q}_{0}} \approx 1+\frac{1}{2 V} \int \mathrm{d} \vec{k}\left\{S_{A A}\left|W_{A}\right|^{2}+S_{B B}\left|W_{B}\right|^{2}+2 S_{A B}\left|W_{A} W_{B}\right|\right\} .
$$

Similar to the Eqn. 2.59, we consider an incompressible system with a soft constraint. The free-energy functional $\mathcal{F}_{s}$ of the single chain is, 


$$
\begin{aligned}
\frac{\mathcal{F}_{s}}{n k_{\mathrm{B}} T}= & \left.-\ln \mathcal{Q}_{0}-\ln \frac{\mathcal{Q}}{\mathcal{Q}_{0}}+\frac{1}{V} \int d \vec{r} \chi N \Phi_{A}\left(1-\Phi_{A}\right)-W_{A} \Phi_{A}-W_{B}\left(1-\Phi_{A}\right)\right] \\
\approx & -\ln \mathcal{Q}_{0}-\frac{1}{2 V} \int \mathrm{d} \vec{k} S_{A A}\left|W_{A}\right|^{2}+S_{B B}\left|W_{B}\right|^{2}+2 S_{A B}\left|W_{A} W_{B}\right| \\
& -\frac{\chi N}{4}\left(\Phi_{A}-\Phi_{B}\right)^{2}+\frac{\kappa N}{2}\left(\Phi_{A}+\Phi_{B}-1\right)^{2} \\
& +W_{A} \Phi_{A}+W_{B} \Phi_{B}
\end{aligned}
$$

By the saddle-point approximation, the free energy functional $\frac{\mathcal{F}_{s}}{n k_{\mathrm{B}} T}\left[W_{A}, W_{B}, \Phi_{A}, \Phi_{B}\right]$ gives the linear relation between the density and the field,

$$
\begin{aligned}
-\Phi_{A} & =S_{A A} W_{A}+S_{A B} W_{B} \\
-\Phi_{B} & =S_{A B} W_{A}+S_{B B} W_{B}
\end{aligned}
$$

In the diblock copolymer melts, we consider the composition fluctuation $\left\langle|m(\mathbf{q})|^{2}\right\rangle$ where $m=\Phi_{A}-\Phi_{B}$ and the density fluctuation $\left\langle|\Phi(\mathbf{q})|^{2}\right\rangle$ where $\Phi=\Phi_{A}+\Phi_{B}$.

We obtain that,

$$
\begin{aligned}
W_{A} & =\frac{m\left(S_{A A}+S_{A B}\right)-\Phi\left(S_{A B}-S_{B B}\right)}{2\left(S_{A A}^{2}-S_{A A} S_{B B}\right)} \\
W_{B} & =\frac{\Phi\left(S_{A A}-S_{A B}\right)-m\left(S_{A A}+S_{A B}\right)}{2\left(S_{A A}^{2}-S_{A A} S_{B B}\right)}
\end{aligned}
$$

The free energy functional $\frac{\mathcal{F}_{s}}{k_{\mathrm{B}} T}\left[W_{A}, W_{B}, \Phi_{A}, \Phi_{B}\right]$ is substituted by these saddlepoint terms,

$$
\begin{aligned}
& \frac{1}{\sqrt{\overline{\mathcal{N}}}} \frac{\mathcal{F}_{s}}{k_{\mathrm{B}} T}=\int \mathrm{d} \mathbf{q} \frac{1}{2}\left(\frac{S_{A A}+2 S_{A B}+S_{B B}}{4\left(S_{A A} S_{B B}-S_{A B}^{2}\right)}-\frac{\chi N}{2}\right)|m|^{2} \\
& +\frac{1}{2}\left(\frac{S_{A A}-2 S_{A B}+S_{B B}}{4\left(S_{A A} S_{B B}-S_{A B}^{2}\right)}+\kappa N\right)|\Phi|^{2} \\
& +\frac{1}{2}\left(\frac{S_{A A}-S_{B B}}{2\left(S_{A A} S_{B B}-S_{A B}^{2}\right)}\right)|m \Phi|^{2}
\end{aligned}
$$

The coupling term between the density fluctuation and the composition fluctuation vanishes when the composition fraction $f_{A}$ equals to 0.5 . We can read off 
the composition structure factor of block copolymers $S_{\text {con }}(\mathbf{q})$,

$$
\frac{N}{S_{\mathrm{con}}(\mathbf{q})}=\frac{4}{n} \frac{V^{2}}{N^{2}} \frac{1}{\left\langle|m(\mathbf{q})|^{2}\right\rangle}=\frac{2}{S_{A A}-S_{A B}}-2 \chi N .
$$

Similarly, we obtain the density fluctuations $S_{\text {tot }}$,

$$
\frac{N}{S_{\mathrm{tot}}(\mathbf{q})}=\frac{4}{n} \frac{V^{2}}{N^{2}} \frac{1}{\left\langle|\Phi(\mathbf{q})|^{2}\right\rangle}=\frac{1}{2\left(S_{A A}+S_{B B}\right)}+\kappa N .
$$

When the incompatible parameter $\chi N$ increases to the order-disorder transition point, the composition fluctuation at a certain value of $\mathbf{q}$ will diverge. This is the spinodal point of symmetric diblock copolymer melts where the homogeneous phase becomes unstable. 


\section{Bibliography}

[1] Michael Rubinstein and Ralph H Colby. Polymer physics, volume 23. Oxford University Press New York, 2003.

[2] Yonghui Deng, Jing Wei, Zhenkun Sun, and Dongyuan Zhao. Large-pore ordered mesoporous materials templated from non-pluronic amphiphilic block copolymers. Chemical Society Reviews, 42(9):4054-4070, 2013.

[3] T Thurn-Albrecht, J Schotter, GA Kästle, N Emley, T Shibauchi, L Krusin-Elbaum, K Guarini, CT Black, MT Tuominen, and TP Russell. Ultrahigh-density nanowire arrays grown in self-assembled diblock copolymer templates. Science, 290(5499):2126-2129, 2000.

[4] Benoit Dubertret, Paris Skourides, David J Norris, Vincent Noireaux, Ali $\mathrm{H}$ Brivanlou, and Albert Libchaber. In vivo imaging of quantum dots encapsulated in phospholipid micelles. Science, 298(5599):1759-1762, 2002.

[5] Michael R Bockstaller, Yonit Lapetnikov, Shlomo Margel, and Edwin L Thomas. Size-selective organization of enthalpic compatibilized nanocrystals in ternary block copolymer/particle mixtures. Journal of the American Chemical Society, 125(18):5276-5277, 2003.

[6] Fenghua Meng, Zhiyuan Zhong, and Jan Feijen. Stimuli-responsive polymersomes for programmed drug delivery. Biomacromolecules, 10(2): 197-209, 2009.

[7] Ricardo Ruiz, Huiman Kang, François A Detcheverry, Elizabeth Dobisz, Dan S Kercher, Thomas R Albrecht, Juan J de Pablo, and Paul F Nealey. Density multiplication and improved lithography by directed block copolymer assembly. Science, 321(5891):936-939, 2008. 
[8] Frank S Bates, Glenn H Fredrickson, Dennis Hucul, and Stephen F Hahn. Pche-based pentablock copolymers: Evolution of a new plastic. AIChE journal, 47(4):762-765, 2001.

[9] Sangwoo Lee, Michael J Bluemle, and Frank S Bates. Discovery of a frank-kasper $\sigma$ phase in sphere-forming block copolymer melts. Science, 330(6002):349-353, 2010.

[10] Meijiao Liu, Yicheng Qiang, Weihua Li, Feng Qiu, and An-Chang Shi. Stabilizing the frank-kasper phases via binary blends of ab diblock copolymers. ACS Macro Letters, 5(10):1167-1171, 2016.

[11] Frank S Bates, Marc A Hillmyer, Timothy P Lodge, Christopher M Bates, Kris T Delaney, and Glenn H Fredrickson. Multiblock polymers: panacea or pandora's box? Science, 336(6080):434-440, 2012.

[12] Nikos Hadjichristidis, Marinos Pitsikalis, Stergios Pispas, and Hermis Iatrou. Polymers with complex architecture by living anionic polymerization. Chemical reviews, 101(12):3747-3792, 2001.

[13] Kurt Kremer and Florian Müller-Plathe. Multiscale problems in polymer science: simulation approaches. MRS bulletin, 26(3):205-210, 2001.

[14] Michele Perego, Federico Ferrarese Lupi, Monica Ceresoli, Tommaso Jacopo Giammaria, Gabriele Seguini, Emanuele Enrico, Luca Boarino, Diego Antonioli, Valentina Gianotti, Katia Sparnacci, et al. Ordering dynamics in symmetric ps-b-pmma diblock copolymer thin films during rapid thermal processing. Journal of Materials Chemistry C, 2 (32):6655-6664, 2014.

[15] Chuyang Y Tang, Young-Nam Kwon, and James O Leckie. Effect of membrane chemistry and coating layer on physiochemical properties of thin film composite polyamide ro and nf membranes: I. ftir and xps characterization of polyamide and coating layer chemistry. Desalination, 242(1-3):149-167, 2009.

[16] Peter F Green and Ratchana Limary. Block copolymer thin films: pattern formation and phase behavior. Advances in Colloid and interface Science, 94 (1):53-81, 2001. 
[17] Mark W Matsen and Michael Schick. Stable and unstable phases of a diblock copolymer melt. Physical Review Letters, 72(16):2660, 1994.

[18] Yiyong Mai and Adi Eisenberg. Self-assembly of block copolymers. Chemical Society Reviews, 41(18):5969-5985, 2012.

[19] Ludwik Leibler. Theory of microphase separation in block copolymers. Macromolecules, 13(6):1602-1617, 1980.

[20] Frank S Bates and Glenn H Fredrickson. Block copolymer thermodynamics: theory and experiment. Annual review of physical chemistry, 41(1):525-557, 1990.

[21] Frank S Bates, Jeffrey H Rosedale, and Glenn H Fredrickson. Fluctuation effects in a symmetric diblock copolymer near the order-disorder transition. The Journal of Chemical Physics, 92(10):6255-6270, 1990.

[22] JGEM Fraaije, BAC Van Vlimmeren, NM Maurits, M Postma, OA Evers, C Hoffmann, P Altevogt, and G Goldbeck-Wood. The dynamic mean-field density functional method and its application to the mesoscopic dynamics of quenched block copolymer melts. The Journal of chemical physics, 106(10):4260-4269, 1997.

[23] Cheng-Zhong Zhang and Zhen-Gang Wang. Random isotropic structures and possible glass transitions in diblock copolymer melts. Physical Review E, 73(3):031804, 2006.

[24] Pablo G Debenedetti and Frank H Stillinger. Supercooled liquids and the glass transition. Nature, 410(6825):259-267, 2001.

[25] Ludovic Berthier and Giulio Biroli. Theoretical perspective on the glass transition and amorphous materials. Reviews of Modern Physics, 83(2): $587,2011$.

[26] B Frick and D Richter. The microscopic basis of the glass transition in polymers from neutron scattering studies. Science, 267(5206):1939, 1995.

[27] RG Petschek and Haria Metiu. A computer simulation of the time-dependent ginzburg-landau model for spinodal decomposition. The Journal of chemical physics, 79(7):3443-3456, 1983. 
[28] Christopher Harrison, Douglas H Adamson, Zhengdong Cheng, John M Sebastian, Srinivasan Sethuraman, David A Huse, Richard A Register, and PM Chaikin. Mechanisms of ordering in striped patterns. Science, 290(5496):1558-1560, 2000.

[29] Weihua Li and Marcus Müller. Defects in the self-assembly of block copolymers and their relevance for directed self-assembly. Annual review of chemical and biomolecular engineering, 6:187-216, 2015.

[30] SB Darling. Directing the self-assembly of block copolymers. Progress in Polymer Science, 32(10):1152-1204, 2007.

[31] Hanqiong Hu, Manesh Gopinadhan, and Chinedum O Osuji. Directed self-assembly of block copolymers: a tutorial review of strategies for enabling nanotechnology with soft matter. Soft matter, 10(22):3867-3889, 2014.

[32] Zhong-Ren Chen, Julia A Kornfield, Steven D Smith, Jeffrey T Grothaus, and Michael M Satkowski. Pathways to macroscale order in nanostructured block copolymers. Science, 277(5330):1248-1253, 1997.

[33] Shinichi Sakurai. Progress in control of microdomain orientation in block copolymers-efficiencies of various external fields. Polymer, 49(12): 2781-2796, 2008.

[34] Karl Amundson, Eugene Helfand, XN Quan, Steven D Hudson, Steven D Smith, et al. Alignment of lamellar block-copolymer microstructure in an electric-field. 2. mechanisms of alignment. Macromolecules, 27(22):6559-6570, 1994.

[35] TL Morkved, M Lu, AM Urbas, EE Ehrichs, et al. Local control of microdomain orientation in diblock copolymer thin films with electric fields. Science, 273(5277):931, 1996.

[36] U Welling, M Muller, H Shalev, and Y Tsori. Block copolymer ordering in cylindrical capacitors. Macromolecules, 47(5):1850-1864, 2014.

[37] Ion Bita, Joel KW Yang, Yeon Sik Jung, Caroline A Ross, Edwin L Thomas, and Karl K Berggren. Graphoepitaxy of self-assembled block copolymers on two-dimensional periodic patterned templates. Science, 321(5891):939-943, 2008. 
[38] Roel Gronheid, Paulina Rincon Delgadillo, Hari Pathangi, Dieter Van den Heuvel, Doni Parnell, Boon Teik Chan, Yu-Tsung Lee, Lieve Van Look, Yi Cao, YoungJun Her, et al. Defect reduction and defect stability in imec's $14 \mathrm{~nm}$ half-pitch chemo-epitaxy dsa flow. In Alternative Lithographic Technologies VI, volume 9049, page 904905. International Society for Optics and Photonics, 2014.

[39] E Weinan, Weiqing Ren, and Eric Vanden-Eijnden. String method for the study of rare events. Physical Review B, 66(5):052301, 2002.

[40] John Edward Jones. On the determination of molecular fields. ii. from the equation of state of a gas. In Proceedings of the Royal Society of London A: Mathematical, Physical and Engineering Sciences, volume 106, pages 463-477. The Royal Society, 1924.

[41] Walter Selke. Monte carlo and molecular dynamics of condensed matter systems. Journal of Statistical Physics, 87(3):959-960, 1997.

[42] Christian Lubich. From quantum to classical molecular dynamics: reduced models and numerical analysis. European Mathematical Society, 2008.

[43] Jacob N Israelachvili. Intermolecular and surface forces. Academic press, 2011.

[44] Patrick Ilg, Vlasis Mavrantzas, and Hans Christian Öttinger. Multiscale modeling and coarse graining of polymer dynamics: simulations guided by statistical beyond-equilibrium thermodynamics. arXiv preprint arXiv:0911.1001, 2009.

[45] Jörg Baschnagel, Kurt Binder, Pemra Doruker, Andrei Gusev, Oliver Hahn, Kurt Kremer, Wayne Mattice, Florian Müller-Plathe, Michael Murat, Wolfgang Paul, et al. Bridging the gap between atomistic and coarse-grained models of polymers: Status and perspectives. Viscoelasticity, atomistic models, statistical chemistry, pages 41-156, 2000.

[46] Marcus Müller. Studying amphiphilic self-assembly with soft coarse-grained models. Journal of Statistical Physics, 145(4):967-1016, 2011.

[47] Pierre-Gilles De Gennes. Scaling concepts in polymer physics. Cornell university press, 1979. 
[48] NM Maurits and JGEM Fraaije. Mesoscopic dynamics of copolymer melts: from density dynamics to external potential dynamics using nonlocal kinetic coupling. The Journal of chemical physics, 107(15): 5879-5889, 1997.

[49] Paul J Flory. Thermodynamics of high polymer solutions. The Journal of chemical physics, 10(1):51-61, 1942.

[50] Paul J Flory and William R Krigbaum. Thermodynamics of high polymer solutions. Annual review of physical chemistry, 2(1):383-402, 1951.

[51] Maurice L Huggins. Thermodynamic properties of solutions of long-chain compounds. Annals of the New York Academy of Sciences, 43(1): $1-32,1942$.

[52] H Tompa. Phase relationships in polymer solutions. Transactions of the Faraday Society, 45:1142-1152, 1949.

[53] Nan Xie, Weihua Li, Hongdong Zhang, Feng Qiu, and An-Chang Shi. Kinetics of lamellar formation on sparsely stripped patterns. The Journal of chemical physics, 139(19):194903, 2013.

[54] Takao Ohta and Kyozi Kawasaki. Equilibrium morphology of block copolymer melts. Macromolecules, 19(10):2621-2632, 1986.

[55] John W Cahn and John E Hilliard. Free energy of a nonuniform system. i. interfacial free energy. The Journal of chemical physics, 28(2):258-267, 1958.

[56] Masao Doi and Samuel Frederick Edwards. The theory of polymer dynamics, volume 73. oxford university press, 1988.

[57] Marcus Müller and Jiuzhou Tang. Alignment of copolymer morphology by planar step elongation during spinodal self-assembly. Physical review letters, 115(22):228301, 2015.

[58] Glenn H Fredrickson and Eugene Helfand. Fluctuation effects in the theory of microphase separation in block copolymers. The Journal of Chemical Physics, 87(1):697-705, 1987.

[59] Jeffrey R Kuhn and Thomas D Pollard. Real-time measurements of actin filament polymerization by total internal reflection fluorescence microscopy. Biophysical journal, 88(2):1387-1402, 2005. 
[60] Prince E Rouse Jr. A theory of the linear viscoelastic properties of dilute solutions of coiling polymers. The Journal of Chemical Physics, 21(7): 1272-1280, 1953.

[61] P Mazur. On the theory of brownian motion. Physica, 25(1-6):149-162, 1959.

[62] Daan Frenkel and Berend Smit. Understanding molecular simulation: from algorithms to applications, volume 1. Academic press, 2001.

[63] PJ Rossky, JD Doll, and HL Friedman. Brownian dynamics as smart monte carlo simulation. The Journal of Chemical Physics, 69(10):4628-4633, 1978.

[64] Marcus Müller and Kostas Ch Daoulas. Single-chain dynamics in a homogeneous melt and a lamellar microphase: A comparison between smart monte carlo dynamics, slithering-snake dynamics, and slip-link dynamics. The Journal of chemical physics, 129(16):164906, 2008.

[65] Mohamed Laradji, Hong Guo, and Martin J Zuckermann. Off-lattice monte carlo simulation of polymer brushes in good solvents. Physical Review E, 49(4):3199, 1994.

[66] Ling Miao, Hong Guo, and Martin J Zuckermann. Conformation of polymer brushes under shear: chain tilting and stretching. Macromolecules, 29(6):2289-2297, 1996.

[67] François A Detcheverry, Darin Q Pike, Paul F Nealey, Marcus Müller, and Juan J de Pablo. Monte carlo simulation of coarse grain polymeric systems. Physical review letters, 102(19):197801, 2009.

[68] Kostas Ch Daoulas and Marcus Müller. Single chain in mean field simulations: Quasi-instantaneous field approximation and quantitative comparison with monte carlo simulations. The Journal of chemical physics, 125(18):184904, 2006.

[69] Mark E Tuckerman, Bruce J Berne, and Glenn J Martyna. Molecular dynamics algorithm for multiple time scales: Systems with long range forces. The Journal of chemical physics, 94(10):6811-6815, 1991. 
[70] MBBJM Tuckerman, Bruce J Berne, and Glenn J Martyna. Reversible multiple time scale molecular dynamics. The Journal of chemical physics, 97(3):1990-2001, 1992.

[71] NVIDIA CORPORATION. NCCL 1.0. URL

https://github.com/NVIDIA/nccl.

[72] Marshall Fixman. Excluded volume in polymer chains. The Journal of Chemical Physics, 23(9):1656-1659, 1955.

[73] Sam F Edwards. The statistical mechanics of polymers with excluded volume. Proceedings of the Physical Society, 85(4):613, 1965.

[74] M Muthukumar and Bernie G Nickel. Perturbation theory for a polymer chain with excluded volume interaction. The Journal of chemical physics, 80(11):5839-5850, 1984.

[75] M Muthukumar and BG Nickel. Expansion of a polymer chain with excluded volume interaction. The Journal of chemical physics, 86(1): 460-476, 1987.

[76] ON Vassiliev and MW Matsen. Fluctuation effects in block copolymer melts. The Journal of chemical physics, 118(16):7700-7713, 2003.

[77] David P Landau and Kurt Binder. A guide to Monte Carlo simulations in statistical physics. Cambridge university press, 2014.

[78] Péter Pulay. Convergence acceleration of iterative sequences. the case of scf iteration. Chemical Physics Letters, 73(2):393-398, 1980.

[79] Richard A Friesner. Solution of self-consistent field electronic structure equations by a pseudospectral method. Chemical physics letters, 116(1): 39-43, 1985.

[80] Scott W Sides and Glenn H Fredrickson. Parallel algorithm for numerical self-consistent field theory simulations of block copolymer structure. Polymer, 44(19):5859-5866, 2003.

[81] Saul A Teukolsky. Stability of the iterated crank-nicholson method in numerical relativity. Physical Review D, 61(8):087501, 2000. 
[82] Clive Fletcher. Computational techniques for fluid dynamics 2: Specific techniques for different flow categories. Springer Science \& Business Media, 2012.

[83] G Tzeremes, KØ Rasmussen, T Lookman, and A Saxena. Efficient computation of the structural phase behavior of block copolymers. Physical Review E, 65(4):041806, 2002.

[84] Kris T Delaney and Glenn H Fredrickson. Polymer field-theory simulations on graphics processing units. Computer Physics Communications, 184(9):2102-2110, 2013.

[85] Parallel Versions of FFTW. URL http://www.fftw.org/parallel/parallel-fftw.html.

[86] cufft. URL https://developer.nvidia.com/cufft.

[87] Donald R Paul. Polymer blends, volume 1. Elsevier, 2012.

[88] AN Semenov. Theory of block copolymer interfaces in the strong segregation limit. Macromolecules, 26(24):6617-6621, 1993.

[89] Marcus Müller, Kostas Ch Daoulas, and Yuki Norizoe. Computing free energies of interfaces in self-assembling systems. Physical Chemistry Chemical Physics, 11(12):2087-2097, 2009.

[90] YG Smirnova and M Müller. Calculation of membrane bending rigidity using field-theoretic umbrella sampling. The Journal of chemical physics, 143(24):243155, 2015.

[91] Fugao Wang and DP Landau. Efficient, multiple-range random walk algorithm to calculate the density of states. Physical review letters, 86(10): 2050, 2001.

[92] Hector D Ceniceros and Glenn H Fredrickson. Numerical solution of polymer self-consistent field theory. Multiscale Modeling $\mathcal{E}$ Simulation, 2 (3):452-474, 2004.

[93] De-Wen Sun and Marcus Müller. Process-accessible states of block copolymers. Physical Review Letters, 118(6):067801, 2017. 
[94] JGEM Fraaije. Dynamic density functional theory for microphase separation kinetics of block copolymer melts. The Journal of chemical physics, 99(11):9202-9212, 1993.

[95] Glenn H Fredrickson. Dynamics and rheology of inhomogeneous polymeric fluids: A complex langevin approach. The Journal of chemical physics, 117(14):6810-6820, 2002.

[96] Marc Souaille and Benoit Roux. Extension to the weighted histogram analysis method: combining umbrella sampling with free energy calculations. Computer physics communications, 135(1):40-57, 2001.

[97] Pierre C Hohenberg and Bertrand I Halperin. Theory of dynamic critical phenomena. Reviews of Modern Physics, 49(3):435, 1977.

[98] David S Dean. Langevin equation for the density of a system of interacting langevin processes. Journal of Physics A: Mathematical and General, 29(24):L613, 1996.

[99] Umberto Marini Bettolo Marconi and Pedro Tarazona. Dynamic density functional theory of fluids. The Journal of Chemical Physics, 110(16): 8032-8044, 1999.

[100] Ryuichi Hasegawa and Masao Doi. Adsorption dynamics. extension of self-consistent field theory to dynamical problems. Macromolecules, 30 (10):3086-3089, 1997.

[101] PG De Gennes. Dynamics of fluctuations and spinodal decomposition in polymer blends. The Journal of Chemical Physics, 72(9):4756-4763, 1980.

[102] Ellen Reister, Marcus Müller, and Kurt Binder. Spinodal decomposition in a binary polymer mixture: Dynamic self-consistent-field theory and monte carlo simulations. Physical Review E, 64(4):041804, 2001.

[103] Marcus Müller and Friederike Schmid. Incorporating fluctuations and dynamics in self-consistent field theories for polymer blends. Advanced Computer Simulation Approaches for Soft Matter Sciences II, pages 1-58, 2005.

[104] Xuehao He and Friederike Schmid. Dynamics of spontaneous vesicle formation in dilute solutions of amphiphilic diblock copolymers. Macromolecules, 39(7):2654-2662, 2006. 
[105] Miri Park, Christopher Harrison, Paul M Chaikin, Richard A Register, and Douglas H Adamson. Block copolymer lithography: periodic arrays of $^{\sim} 1011$ holes in 1 square centimeter. Science, 276(5317):1401-1404, 1997.

[106] AE Bailey, Wilson Che Kei Poon, Rebecca J Christianson, Andrew B Schofield, Urs Gasser, Vikram Prasad, Suliana Manley, Phil N Segre, Luca Cipelletti, William V Meyer, et al. Spinodal decomposition in a model colloid-polymer mixture in microgravity. Physical review letters, 99 (20):205701, 2007.

[107] Mark P Stoykovich, Marcus Müller, Sang Ouk Kim, Harun H Solak, Erik W Edwards, Juan J De Pablo, and Paul F Nealey. Directed assembly of block copolymer blends into nonregular device-oriented structures. Science, 308(5727):1442-1446, 2005.

[108] Weihua Li, Meijiao Liu, Feng Qiu, and An-Chang Shi. Phase diagram of diblock copolymers confined in thin films. The Journal of Physical Chemistry B, 117(17):5280-5288, 2013.

[109] M Müller. Concurrent coupling between a particle simulation and a continuum description. The European Physical Journal-Special Topics, 177 (1):149-164, 2009.

[110] Marcus Müller and Kostas Ch Daoulas. Speeding up intrinsically slow collective processes in particle simulations by concurrent coupling to a continuum description. Physical review letters, 107(22):227801, 2011.

[111] Marcus Müller and Juan J de Pablo. Computational approaches for the dynamics of structure formation in self-assembling polymeric materials. Annual Review of Materials Research, 43:1-34, 2013.

[112] John W Cahn. Phase separation by spinodal decomposition in isotropic systems. The Journal of Chemical Physics, 42(1):93-99, 1965.

[113] HE Cook. Brownian motion in spinodal decomposition. Acta metallurgica, 18(3):297-306, 1970.

[114] Toshihiro Kawakatsu. Effects of changes in the chain conformation on the kinetics of order-disorder transitions in block copolymer melts. Physical Review E, 56(3):3240, 1997. 
[115] Chuck Yeung and An-Chang Shi. Formation of interfaces in incompatible polymer blends: A dynamical mean field study. Macromolecules, 32(11):3637-3642, 1999.

[116] Takashi Uneyama. Density functional simulation of spontaneous formation of vesicle in block copolymer solutions. The Journal of chemical physics, 126(11):114902, 2007.

[117] Xinghua Zhang, Shuanhu Qi, and Dadong Yan. Spinodal assisted growing dynamics of critical nucleus in polymer blends. The Journal of chemical physics, 137(18):184903, 2012.

[118] IlyaM Lifshitz and VitalyV Slyozov. The kinetics of precipitation from supersaturated solid solutions. Journal of physics and chemistry of solids, 19 (1-2):35-50, 1961.

[119] Kyozi Kawasaki and Ken Sekimoto. Concentration dynamics in polymer blends and block copolymer melts. Macromolecules, 22(7):3063-3075, 1989.

[120] F Brochard and PG De Gennes. Dynamics of compatible polymer mixtures. Physica A: Statistical Mechanics and its Applications, 118(1-3): 289-299, 1983.

[121] I Ya Erukhimovich and AN Semenov. Nonexponential density relaxation and the dynamic form-factor of polymer melts in the reptation regime. Zh. Eksp. Teor. Fiz, 63:275, 1986.

[122] PE Ramirez-Gonzalez and M Medina-Noyola. Glass transition in soft-sphere dispersions. Journal of Physics: Condensed Matter, 21(7): 075101, 2009.

[123] Pedro Ramírez-González and Magdaleno Medina-Noyola. General nonequilibrium theory of colloid dynamics. Physical Review E, 82(6): 061503, 2010.

[124] Shuanhu Qi and Friederike Schmid. Dynamic density functional theories for inhomogeneous polymer systems compared to brownian dynamics simulations. Macromolecules, 50(24):9831-9845, 2017. 
[125] E Weinan, Weiqing Ren, and Eric Vanden-Eijnden. Simplified and improved string method for computing the minimum energy paths in barrier-crossing events. The Journal of Chemical Physics, 2007.

[126] Hassei Takahashi, Nabil Laachi, Kris T Delaney, Su-Mi Hur, Corey J Weinheimer, David Shykind, and Glenn H Fredrickson. Defectivity in laterally confined lamella-forming diblock copolymers: thermodynamic and kinetic aspects. Macromolecules, 45(15):6253-6265, 2012.

[127] Xiuyuan Cheng, Ling Lin, E Weinan, Pingwen Zhang, and An-Chang Shi. Nucleation of ordered phases in block copolymers. Physical review letters, 104(14):148301, 2010.

[128] Samuel M Allen and John W Cahn. A microscopic theory for antiphase boundary motion and its application to antiphase domain coarsening. Acta Metallurgica, 27(6):1085-1095, 1979.

[129] Abraham Nitzan. Activated rate processes in condensed phases: The kramers theory revisited. Advances in Chemical Physics: Evolution of Size Effects in Chemical Dynamics, Part 2, Volume 70, pages 489-555, 1988.

[130] Weihua Li, Paul F Nealey, Juan J de Pablo, and Marcus Müller. Defect removal in the course of directed self-assembly is facilitated in the vicinity of the order-disorder transition. Physical review letters, 113(16): 168301, 2014.

[131] Zhen-Gang Wang. Response and instabilities of the lamellar phase of diblock copolymers under uniaxial stress. The Journal of chemical physics, 100(3):2298-2309, 1994.

[132] BA Garetz, NP Balsara, HJ Dai, Z Wang, MC Newstein, and B Majumdar. Orientation correlations in lamellar block copolymers. Macromolecules, 29(13):4675-4679, 1996.

[133] Ricardo Ruiz, Robert L Sandstrom, and Charles T Black. Induced orientational order in symmetric diblock copolymer thin films. Advanced Materials, 19(4):587-591, 2007.

[134] R Ruiz, JK Bosworth, and CT Black. Effect of structural anisotropy on the coarsening kinetics of diblock copolymer striped patterns. Physical Review B, 77(5):054204, 2008. 
[135] Kristel Michielsen and Hans De Raedt. Integral-geometry morphological image analysis. Physics Reports, 347(6):461-538, 2001.

[136] H. Mantz, K. Jacobs, and K. Mecke. Utilizing minkowski functionals for image analysis: A marching square algorithm. Journal of Statistical Mechanics-Theory and Experiment, P:12015, 2008.

[137] M. W. Matsen. The standard Gaussian model for block copolymer melts. 14:R21-R47, 2002.

[138] M. Peach and J. S. Koehler. The forces exerted on dislocations and the stress fields produced by them. Phys. Rev., 80:436-439, 1950. doi: 10.1103/PhysRev.80.436. URL http://link.aps.org/doi/10.1103/PhysRev.80.436.

[139] Weihua Li and Marcus Müller. Thermodynamics and kinetics of defect motion and annihilation in the self-assembly of lamellar diblock copolymers. Macromolecules, 49(16):6126-6138, 2016. doi: 10.1021/acs.macromol.6b01088.

[140] J. L. Barrat and G. H. Fredrickson. Diffusion of a symmetrical block copolymer in a periodic potential. 24:6378-6383, 1991.

[141] T. P. Lodge and M. C. Dalvi. Mechanisms of chain diffusion in lamellar block-copolymers. 75:657-660, 1995.

[142] J Weertman. The peach-koehler equation for the force on a dislocation modified for hydrostatic pressure. Philosophical Magazine, 11(114): 1217-1223, 1965.

[143] Hai Qian and Gene F Mazenko. Defect structures in the growth kinetics of the swift-hohenberg model. Physical Review E, 67(3):036102, 2003. 


\section{Curriculum Vitae}

\section{Personal Data}

Name : $\quad$ Ren, Yongzhi

Date of birth : 28.05.1987

Place of birth: Heilongjiang, China

Nationality: China

Address : $\quad$ Zimmermannstr. 14, 37075, Göttingen

\section{Education}

10.2013- PhD student in the group of Prof. Dr. Marcus Müller, Institute of theoretical physics, Georg-August-Universität Göttingen

09.2010-06.2013 Master of Science (theoretical physics), Department of Physics,

Renmin university of China (China)

09.2016-06.2010 Bachelor of Mathematics and Applied Mathematics, School of Mathematics and Statistics, Central South University (China) 